\title{
Photonic Modulation and Demodulation techniques for Multi-Gb/s Millimetre-wave Wireless Links
}

\author{
Rakesh Sambaraju
}

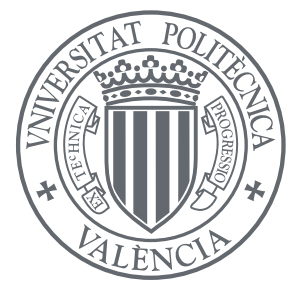

València 2010

UPV-PHD-2010-3407 
Universitat Politècnica de València I.U.I Nanofotonica

Camí de Vera S/N,Ed. 8F, 46022 Valencia, Spain.

Phone +34 963879746, Fax +45 45882673

info@ntc.upv.es

www.ntc.upv.es 


\section{Abstract}

Radio over fibre (RoF) systems offer the bandwidth and flexibility for generation and distribution of future wireless access standards in an optical access architecture, which reduces the cost of the networks by centralising signal processing, and simplifying remote antenna sites. The use of optical communication systems as transport medium in RoF links is reducing the current access bottle neck between the wireless access standards and current wired access in a converged optical domain. The optical access networks are moving towards $10 \mathrm{~Gb} / \mathrm{s}$ capacity with standards like 10GEPON, leaving a bottle neck between the wireless and optical access. This has led to a great research efforts in generation and distribution of high capacity $(10 \mathrm{~Gb} / \mathrm{s}$ and more) wireless links using RoF.

In this thesis the use of photonic techniques for generation, distribution, and detection of high capacity vector modulated wireless signals is investigated. This thesis is mainly dedicated to the photonic generation of advanced spectral efficient wireless signals with modulation formats like quadrature phase shift keying (QPSK), multi-level quadrature amplitude modulation (M-QAM). The work presented in thesis is categorised in two parts: The first part deals with photonic techniques involving electrical coherent techniques for both modulation and demodulation of wireless signals, whereas the second part contains all optical incoherent techniques for modulation of demodulation of wireless signals. 
In the first part of this thesis, several system architectures for generation and detection of wireless signals are presented, which are numerically evaluated and experimentally demonstrated. A novel concept called photonic vector modulation (PVM) for generation of multi-level quadrature amplitude modulated (M-QAM) millimetre wave wireless signals is proposed. Based on this technique, signal generation of $10 \mathrm{~Gb} / \mathrm{s} 16-\mathrm{QAM}$ and QPSK wireless is presented in this thesis. Performance of the wireless signals generated using PVM is analysed in a reconfigurable WDM network. Based on the similar concept a demodulation technique for $\mathrm{M}-$ QAM wireless signals named photonic vector demodulation (PVdM) is presented. Both the PVM and PVdM techniques use electrical coherent techniques. The use of $\mathrm{I} / \mathrm{Q}$ optical modulator for generation of dispersion tolerant differential phase shift keyed (DxPSK) and use of passive all optical demodulation of DxPSK signals using 1-bit delay Mach-Zehnder interferometer (DMZI) is investigated.

In the second part of the thesis, a novel demodulation technique of wireless signals, which is both dispersion tolerant and radio frequency transparent, using optical coherent detection and digital signal processing is presented. The use of digital signal processing and homo-dyne coherent detection for high speed optical communication systems led to investigation of new modulation technique based on optical heterodyne mixing of free running lasers. Using this technique, wireless signal at record capacities of $20 \mathrm{~Gb} / \mathrm{s}$ using QPSK modulation in the $75-110 \mathrm{GHz}$ band is presented. Based on the similar technique, use of optical OFDM used in optical baseband links, for generation of high capacity wireless is also investigated, and wireless signal generation of up to $40 \mathrm{~Gb} / \mathrm{s}$ using OFDM-QPSK modulation is addressed.

The main outcome of the work presented in this thesis are listed as follows:

- A $10 \mathrm{~Gb} / \mathrm{s}$ QPSK indoor wireless link demonstration in the $60 \mathrm{GHz}$ band using photonic vector modulation.

- Non coherent demodulation technique transparent to RF frequency, and tolerant to dispersion based on optical coherent detection and digital signal processing. 
- QPSK wireless signal generation based on heterodyne mixing of free-running lasers.

- Optical OFDM based $40 \mathrm{~Gb} / \mathrm{s}$ wireless signal generation and coherent detection based demodulation. 


\section{Resumen}

Las sistemas de radio sobre fibra (Radio over fibre ROF) ofrecen el ancho de banda y flexibilidad necesario para la generación y distribución de del señales inalámbricas del futuro en una arquitectura de red óptica, que reduce el coste de las redes centralizando el procesado y simplificando la ubicación de la antena (estación de base EB). El uso de sistemas de comunicaciones ópticas como una media de transporte de señales inalámbricas en enlaces RoF reduce el cuello de botella entre los estándares de acceso inalámbrico y cableado en un dominio convergente óptico. Las redes de acceso ópticas están evolucionando con capacidades de hasta $10 \mathrm{~Gb} / \mathrm{s}$ con el estándar 10GEPON, dejando un cuello de botella entre tecnologías de acceso inalámbrico y óptico. Eso ha motivado gran esfuerzo de investigación en la generación y distribución de señales inalámbricas de alta capacidad (>10 Gb/s) basada en RoF.

En esta tesis ha investigado el uso de técnicas fotónicas para la generación, distribución y demodulación de señales inalámbricas moduladas vectorialmente. Este tesis esta principalmente dedicada a la generación de señales inalámbricas espectralmente eficientes como la modulación de fase en cuadratura (QPSK) o modulación de amplitud cuadratura de multinivel (M-QAM). El trabajo presentado en este tesis está clasificado en dos partes: la primera de ellas trata de la técnicas fotónicas que utilizan señales eléctricas coherente para le generación y demodulación de señales inalámbricas, mientras la segunda parte trata de usar señales 
ópticas incoherentes. En la primera parte de la tesis, están presentadas diferentes arquitecturas de sistema y están analizada numéricamente, y demostradas experimentalmente. Un nuevo concepto denominada "modulación vectorial fotónica" (PVM) es propuesta para la generación de señales inalámbricas con una modulación M-QAM. Basando en esta técnica se presenta la generación de señales de capacidad $10 \mathrm{~Gb} / \mathrm{s}$ con una modulación de QPSK y 16-QAM. La comportamiento de dicho señales en una red WDM óptica reconfigurable se ha analizado. Basando en mismo concepto de modulación, se presenta una técnica de demodulación de señales vectoriales M-QAM. Así también investiga el uso de un modulador óptico I/Q para la generación de señales inalámbricas con modulación de fase diferencial, y el uso de un interferómetro óptico (DMZI) para su demodulación.

En la segunda parte de la tesis se investiga el uso de detección coherente óptica para aplicaciones de radio sobre fibra. El procesado digital de señales y el detección coherente óptica permite el batido heterodino de láseres para generación de señales inalámbricas vectoriales. Utilizando este técnica heterodino, se presenta la generación de señales QPSK con una capacidad de hasta $20 \mathrm{~Gb} / \mathrm{s}$ en la banda milimétrica de 75-110 GHz. Así mismo se investiga el uso de técnicas OFDM óptica para la generación de señales de muy alta capacidad y presenta resultados de generación de señales con una capacidad de $40 \mathrm{~Gb} / \mathrm{s}$.

Los resultados principales de esta tesis están detallados a continuación:

- Demostración de una enlace inalámbrico con capacidad de $10 \mathrm{~Gb} / \mathrm{s}$ en la banda de $60 \mathrm{GHz}$ usando la técnica de modulación fotónica vectorial.

- Técnicas de demodulación incoherentes trasparente a la frecuencia RF, y tolerantes a la dispersión cromática de la fibra óptica.

- Generación de señales inalámbricas QPSK usando el batido heterodino de láseres incoherentes.

- Generación de señales inalámbricas basadas en técnicas de OFDM óptica con velocidades hasta $40 \mathrm{~Gb} / \mathrm{s}$. 


\section{Resum}

Els sistemes radio sobre fibra (del anglés Radio over Fibre, RoF) ofereixen el ampli de banda i flexibilitat necessaris per a la generació i distribució dels futurs estàndards d'accés inal-làmbrics en una arquitectura d'accés óptica, el que redueix el cost de les xarxes al centralitzar el procés de senyal, i simplificant l'equipament a la ubicació de l'antena. L'ús dels sistemes de comunicacions òptiques com a mig de transport en els enllaços RoF, redueix el coll de botella d'accés entre els estàndards d'accés inal-làmbrics i els actual accessos per cable en un domini convergent òptic. Les xarxes d'accés òptiques estan desplaçant-se cap a capacitats de 10 Gb/s amb estàndards com 10GEPON, deixant un coll de botella entre els accessos inal-làmbrica i òptic. Açò ha motivat els esforços en la generació i distribució de gran capacitat (10Gb/s i més) dels enllaços inal-làmbrics utilitzant RoF.

En aquesta tesis, s'ha investigat l'ús de tècniques fotòniques per a la generació, distribució i detecció de senyals Inal-làmbriques modulades vectorialment . Aquesta tesis està principalment dedicada a la generació de senyals inal-làmbriques espectralment eficients amb formats de modulació com el desplaçament de fase en quadratura (de langlés quadrature phase shift keying, QPSK), modulació per amplitud multi-nivell en quadratura (de l'anglés multi-level quadrature amplitude, M-QAM). El treball presentat en la tesis està dividit en dos parts: la primera delles tracta les tècniques fotòniques que utilitzen senyals elèctriques coherents tant per a 
la modulació com per a la demodulació, mentre que la segona part tracta l'ús de senyals completament òptiques sense la necessitat de coherència.

En la primera part de la tesis, son presentades diferents arquitectures de sistemes i han sigut avaluades numèricament i demostrades experimentalment. Un nou concepte anomenat modulació vectorial fotònica (PVM) es proposada per a la generació de senyals inal-làmbriques modulades en amplitud de quadratura multi-nivell (M-QAM). Basant-nos en aquesta tècnica es presenta la generació de senyals inalàmbriques de 16-QAM i QPSK de $10 \mathrm{~Gb} / \mathrm{s}$. S'analitzen les prestacions de les senyals inal-làmbriques generades utilitzant PVM en una xarxa WDM reconfigurable . Basant-nos en el mateix concepte es presenta una tècnica de demodulació per a senyals inallàmbriques M-QAM. Així mateix s'investiga l'ús del modulador òptic $\mathrm{I} / \mathrm{Q}$ per a la generació de codificació de fase diferencial tolerant a la dispersió, així com l'ús de la demodulació totalment òptica de senyals DxPSK utilitzant un interferòmetre (DMZI).

En la segona part de la tesis s'investiga l'ús de la detecció coherent òptica digital de les senyals per a la seua demodulació. El processat digital de senyals i les tècniques de detecció coherent permetixen el batut heterodí de làsers per a la generació de senyals inal-làmbriques. Utilitzant esta tècnica, es presenta la generació de senyals inal-làmbriques QPSK amb capacitats fins $20 \mathrm{~Gb} / \mathrm{s}$ en la banda de 75-110 GHz. Basada en la mateixa tècnica es presenta 1 'ús de senyals òptiques OFDM per a la generació de senyals inalàmbriques amb una velocitat binaria de $40 \mathrm{~Gb} / \mathrm{s}$. Els principals resultats d'aquesta tesis son els que es detallen a continuació:

- Demostració d'un enllaç inalàmbric de $10 \mathrm{~Gb} / \mathrm{s}$ de capacitat en la banda de $60 \mathrm{GHz}$ basat en la modulació vectorial fotònica.

- Tècnica de demodulació no coherent transparent a la freqüència RF del senyal tolerant a la dispersió basada en la detecció coherent i en el processat digital del senyal.

- Generació de senyals QPSK inalàmbriques amb batut heterodí de làsers incoherents.

- Generació de senyals inalàmbriques basades en OFDM òptiques amb velocitats fins a $40 \mathrm{~Gb} / \mathrm{s}$. 


\section{Papers included in the thesis}

[A] R. Sambaraju, J. Palaci, V. Polo, and J. L. Corral, "Photonic envelope detector for broadband wireless signals using a single MachZehnder modulator and a fibre Bragg grating," in Proc. 33rd European Conference on Optical Communications, ECOC 2008, paper P 6.4, Brussels Belgium, 2008.

[B] R. Sambaraju, M. A. Piqueras, V.Polo, J. L. Corral, and J. Marti, "Generation of multi-gigabit-per-second MQAM/MPSK-modulated millimeter-wave carriers employing photonic vector modulator techniques," J. Lightw. Technol., vol. 25, no. 11,pp. 3350-3357, 2007.

[C] R. Sambaraju, J. L. Corral, J. Palaci, V. Polo, and J. Marti, "Performance analysis of photonic vector modulation techniques for multi-Gb/s wireless links," J. Lighw. technol., vol. 26, no. 15, pp. 2684-2691, 2008.

[D] J. L. Corral, R. Sambaraju, M. A. Piqueras, and V. Polo, "Generation of pure electrical quadrature amplitude modulation with photonic vector modulator," Opt. Lett., vol. 33, no. 12, pp. 12941296, 2008.

[E] J. L. Corral, R. Sambaraju, M. A. Piqueras, and V. Polo, "Pure $2.5 \mathrm{~Gb} / \mathrm{s} 16-\mathrm{QAM}$ signal generation with photonic vector modulator," in IEEE MTT-S International Microwave Symposium Digest, MTT-S IMS 2008, pp. 595-598, Atlanta GA, 2008. 
[F] R. Sambaraju, M. A. Piqueras, V.Polo, J. L. Corral and J. Marti, "Photonic vector modulation of $3.6 \mathrm{~Gb} / \mathrm{s}$ 16-QAM at $39 \mathrm{GHz}$ for radio-on-fiber systems," in Proc. 32nd European Conference on Optical Communications, ECOC 2007, paper Tu5.4.7, Berlin Germany, 2007.

[G] R. Sambaraju, V. Polo, J. L. Corral, and J. Marti, "Ten gigabits per second 16-level quadrature amplitude modulated millimeterwave carrier generation using dual-drive MachZehnder modulators incorporated photonic vector modulator," Opt. Lett., vol. 33, no. 16, pp. 1833-1835, 2008.

[H] R. Sambaraju, Jose Mora, Beatriz Ortega, and J. L. Corral, "Transmission of optically generated $1.25 \mathrm{~Gb} / \mathrm{s}$ QAM wireless signals in a dynamically reconfigurable optical WDM network," in Proc. Optical Fiber Communication Conf./National Fiber Optics Engineers Conf., OFC/NFOEC 2009 paper OTuJ7, San Diego CA, March 2009.

[I] R. Sambaraju, J. Palaci, R. Alemany, V. Polo, and J. L. Corral, "Photonic vector demodulation of $2.5 \mathrm{Gbit} / \mathrm{s}$ QAM modulated wireless signals," International Topical Meeting on Microwave Photonics, 2008. Jointly held with the 2008 Asia-Pacific Microwave Photonics Conference. MWP/APMP 2008., pp.117-120, Gold Coast Australia, Sept. 2008.

[J] R. Sambaraju, R. Alemany, A. Hakansson, and J. Herrera, "Differential phase modulated hybrid wireless/wireline links based on optical phase modulation and demodulation," to be presented at 2010 IEEE Photonics Society Annual Meeting,paper ThM4, Denver, CO, Nov. 2010.

[K] R. Sambaraju, D. Zibar, R. Alemany, A. Caballero, J. Herrera, and I. T. Monroy, "Radio frequency transparent demodulation for broadband wireless links," in Proc. Optical Fiber Communication Conf./National Fiber Optics Engineers Conf., OFC/NFOEC 2010,paper OML1, San Diego, CA, Mar. 2010.

[L] R. Sambaraju, D. Zibar, A. Caballero, I. T. Monroy, R. Alemany, and J. Herrera, "100 GHz wireless-over-fibre links with up to 16 $\mathrm{Gb} / \mathrm{s}$ QPSK modulation using optical heterodyne generation and 
digital coherent detection," Photon. Lett.,vol. 22, no. 22, pp. 1650-1653, 2010.

[M] R. Sambaraju, D. Zibar, A. Caballero, J. Herrera, J. B. Jensen, I. T. Monroy, U. Westergen,A. Walber, and J. Marti, "Up to 40 $\mathrm{Gb} / \mathrm{s}$ wireless signal generation and demodulation in $75-110 \mathrm{GHz}$ band," International Topical Meetings on Microwave Photonics, MWP 2010,Postdeadline paper, 2010. 


\section{Acknowledgements}

During the process of my Ph.D many have helped me, and here I would like to express my sincere gratitude for their contribution.

First of all I thank my supervisor and director of NTC Prof. Javier Martí for giving me an opportunity to work with him. Also I thank my other supervisor Prof. Juan Luís Corral for his great guidance and support, he has contributed a lot to make me understand the concepts of radio over fibre. In the beginning of my Ph.D work I received a lot of guidance from Valentín and Miguel Àngel who have helped building a foundation to my thesis, I am very grateful to them. Also during the process of my thesis I owe a lot to a few peers and friends like Javi Herrera, Ruben Alemany, Darko Zibar, Idelfonso T. Monroy, Andreas Hakansson, Andreas Stöhr, José Capmany, for showing me the right direction.

Financing my thesis was very crucial for successful completion and i am grateful to NTC and the Genralitat Valenciana for partly funding my work. One of the other entities that indirectly contributed financially towards my thesis is the European Commission. The European projects like FP6 IPHOBAC and FP7 EUROFOS are especially acknowledged where I had a great opportunity of travelling to various conferences and meetings. I also wish to thank my colleagues at DTU in Denmark for their invaluable collaboration and support. 
I am very grateful to all the people who have helped my integration into the Spanish culture of cañas y tapas and I credit that initiative to Antonio, Alfredo and Guillermo. Also they contributed a lot towards my current knowledge on Spanish language, I cannot thank them enough! As time went by there were more people who helped me with cañas and tapas like Ruben, Galan, Jaime, Pixa, Pedro, Ruth and many more, whom I thank for their patience and support.

I also wish to thank the people who have helped with all the paper work involved in the process of Ph.D. I am greatly indebted to Salvador Sales the head of Ph.D studies at the dept. Comunicaciones of UPV for his support. I am also grateful to Ruth for her help. I also thank Rocío the department secretary for her patience and help during the whole process. I also express my sincere gratitude to the distinguished members of the research community, Andreas Stöhr, Nathan Gomes and Valentín Polo for accepting my work.

Last but obviously not the least is my family, that I thank them a lot. I thank my parents for their eternal love and support, and my brother for taking care of me! I also thank Rafa and Maria Angeles for their trust, kindness and love. I am deeply grateful to Angeles for her exceptional cooking everything i visit her!

The only person without whom this would have been impossible is Lucía, I thank you for everything. 


\section{Contents}

Abstract i

Resumen v v

Resum vii

Papers included in the thesis ix

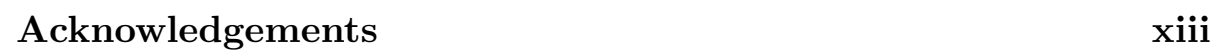

$\begin{array}{lll}1 & \text { Introduction } & 1\end{array}$

1.1 Why photonics? . . . . . . . . . . . . . . . 3

1.2 Photonics for $\mathrm{Gb} / \mathrm{s}$ wireless: State of the art . . . . . . . 5

$1.2 .1 \quad$ Intensity modulated/direct detection (IM/DD) based wireless links ................. 7

1.2.2 Optical heterodyne techniques . . . . . . . . . 9

1.3 Outline of the thesis . . . . . . . . . . . . . . 10

2 Introduction to Radio-Over-Fibre Techniques 11

$2.1 \quad$ Optical Devices . . . . . . . . . . . . . . . 11

2.1 .1 Directly modulated laser . . . . . . . . . . . . . 12

2.1 .2 Mach-Zehnder Modulator . . . . . . . . . . . . . 13

2.1 .3 Electroabsorption modulator . . . . . . . . . . . . 16

$2.1 .4 \quad$ Optical Fibre $\ldots \ldots \ldots \ldots \ldots \ldots$

2.1 .5 Noise Sources. . . . . . . . . . . . . . . . . . 18

2.2 RF Up-conversion Techniques . . . . . . . . . . . . . . . 19 
$2.2 .1 \quad$ Up-conversion using Mach-Zehnder modulator . . 19

2.2.2 Up-conversion Using Electroabsorption Modulator 24

2.3 Radio Over Fibre Uplinks . . . . . . . . . . . . . . . . . . . 26

2.3 .1 Photonic Downconversion . . . . . . . . . . . 27

2.3 .2 Harmonic Distortion . . . . . . . . . . . . . 27

2.3 .3 Envelope Detector [Paper A] . . . . . . . . . . . 30

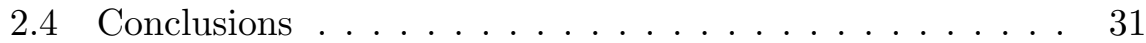

3 Photonic Vector Modulation and Demodulation Techniques 35

3.1 Photonic vector modulation: Working principle. . . . . . . 36

3.1 .1 System Architectures [Paper B] . . . . . . . . . . . 37

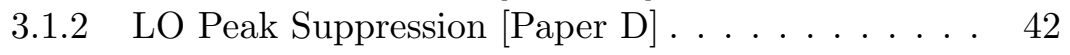

3.1.3 Performance Evaluation [Paper C] . . . . . . . . . . 42

$3.260 \mathrm{GHz}$ Wireless Links $\ldots \ldots \ldots \ldots$. . . . . . . . 46

3.2.1 Link Availability and Link Budget Calculations . . 47

3.2.2 Photonic Vector Modulated $10 \mathrm{~Gb} / \mathrm{s} 60 \mathrm{GHz}$ QPSK Wireless Link . . . . . . . . . . . . . 47

$3.3 \quad$ M-ary Quadrature Amplitude Modulation . . . . . . . . . . 50

3.3.1 16-QAM Wireless Signal Generation [Paper E, F, G] 51

3.4 Distribution of Wireless Signals in a Radio-Over-Fibre

Access Network . . . . . . . . . . . . . . . 53

3.4.1 Dynamic Reconfigurable Optical WDM Network . 54

$3.4 .2 \quad$ Multi service Co-existence [Paper H] . . . . . . . 56

3.5 Photonic Vector Demodulation: Working Principle . . . . 57

$3.5 .1 \quad$ Performance Optimization . . . . . . . . . . . . 59

$3.5 .2 \quad$ Architectures for PvDM [Paper I] $\ldots \ldots \ldots$. . . . 60

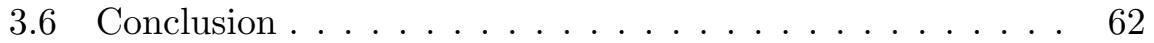

\section{Optical Baseband Modulation for Millimetre Wave Wire-} less 65

4.1 Introduction . . . . . . . . . . . . . . . . . 65

4.2 Optical Baseband Modulation Formats . . . . . . . . . . 66

$4.2 .1 \quad$ DPSK Demodulation . . . . . . . . . . . . . . . 68

$4.2 .2 \quad$ Optical (D)QPSK . . . . . . . . . . . . . . . . . . . . 69

4.3 Integrated Millimetre Wave Modulator Concept . . . . . . 71

4.3 .1 Device Parameter Requirements . . . . . . . . . . 73

4.3.2 Differential Phase Modulated Wireless Links [Pa-

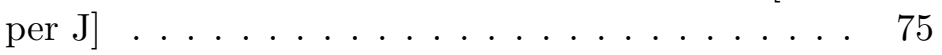

4.4 Optical Digital Coherent Detection . . . . . . . . . . . 77 
4.4 .1 Digital Demodulation. . . . . . . . . . . . . . . 79

4.4.2 Radio over Fibre Demodulating Downlink [Paper K] 81

4.5 Optical Heterodyne Generation of Millimetre Wireless Signals [Paper L] . . . . . . . . . . . . . . . . . . . . . . . 84

4.5 .1 Experimental Validation . . . . . . . . . . . . 86

4.5.2 Optical OFDM based $40 \mathrm{~Gb} / \mathrm{s}$ Wireless Signal Generation $[$ Paper $\mathrm{M}]] \ldots \ldots \ldots$

4.6 Conclusions $\ldots \ldots \ldots \ldots$

5 Conclusions and Future Outlook 93

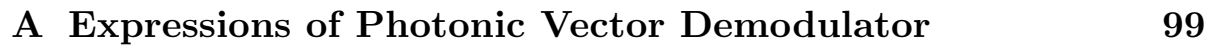


Chapter 1

\section{Introduction}

The society as we know today has been greatly influenced by the development of telecommunications technology and the invention of internet and its multimedia rich applications like YouTube, HULU, Facebook, and others. The Internet has led to the exponential growth in data traffic [1] in telecommunication networks, which in-turn has led to development of new technologies, and vice versa. The majority of telecommunication networks are based on fibre optic communications, which with the invention of Erbium-doped fibre amplifiers (EDFA) 2 has become the favourite technology for long haul data transport [3, 4]. Optical communication systems are widely deployed all over the world, mainly for long haul(> $1000 \mathrm{~km})$ and regional/metro communication links. Until recently, the last mile user connectivity was provided mostly by cable TV, or through twisted pair (ADSL), which have a very limited bandwidth distance product (around $10 \mathrm{Mb} / \mathrm{s}^{*} \mathrm{~km}$ ). The most recent access technology, the hybrid fibre coaxial (HFC) can only provide a few tens of $\mathrm{Mb} / \mathrm{s}$ to each user, and all these technologies do not meet the bandwidth requirements of the future. Recently, fibre optics is entering the last mile segment with the passive optical network (PON) technology also called as fibre to the home (FTTH), where $2.5 \mathrm{~Gb} / \mathrm{s}$ connectivity using a time- 
division-multiplexed PONs [5] are already under implementation. The current state of the art standard for optical access network is a $10 \mathrm{~Gb} / \mathrm{s}$ Ethernet based connectivity called 10GEPON [6], and optical access networks with $40 \mathrm{~Gb} / \mathrm{s}, 7$ are being investigated. This sudden increase in the access networks' capacity is well supported by the advances in metro and core segment where systems for $100 \mathrm{~Gb} / \mathrm{s}[8-12]$ or more $[13$ are being investigated, with a few of them in deployment.

With the rapid growth in optical access networks, there is a digital divide emerging where remote areas, and emerging isolated residential complexes find it hard to have the last mile fibre for either cost or logistical reasons. For example, the cost of leasing a $1 \mathrm{~Gb} / \mathrm{s}$ line can be around 10,000 USD per month, and cost of trenching can be as high as 250,000 USD in metro areas [14. In such cases, wireless access has always played a crucial role in expanding the reach of access networks in a cost-effective manner. On the other hand, wireless access through wireless LANs, WiMAX, LTE, etc are evolving rapidly, and capacity of 100 $\mathrm{Mb} / \mathrm{s}$ per user are already under consideration. But for the wireless technology to support the current optical access, data rates up to $10 \mathrm{~Gb} / \mathrm{s}$ or more are currently needed, for extending the reach of the optical access networks. Some other applications of such high capacity wireless links are inter-building gigabit connectivity, emergency service deployment, redundancy links, etc. A recent survey in the United States [15] revealed that around $90 \%$ of commercial buildings are about 2 miles away from a metro-ring network, and a $\mathrm{Gb} / \mathrm{s}$ wireless technology could potentially connect all these building to the metro network.

To provide multi-Gb/s wireless links, new frequency bands like the millimetre wave $(\mathrm{mmW})$ bands have to be considered [14. The advantages of the $\mathrm{mmW}$ bands are the availability of a few $\mathrm{GHz}$ of bandwidth, which may provide wireless links with Gb/s capacity. Another advantage is the low beam-width of the higher $\mathrm{mmW}$ bands, which in combination with high gain antennas enable higher spatial multiplexing and frequency reuse [16. Of the $\mathrm{mmW}$ band spectrum, the $60 \mathrm{GHz}(57-64 \mathrm{GHz})$ band, and the $70 / 80 \mathrm{GHz}(71-76 / 81-86 \mathrm{GHz})$ are mostly considered by various wireless system developers. The frequency bands of $120 \mathrm{GHz}$ or $140 \mathrm{GHz}$ (both unregulated) are under investigation for very high capacity wireless. To provide $10 \mathrm{~Gb} / \mathrm{s}$ wireless links in these frequency 


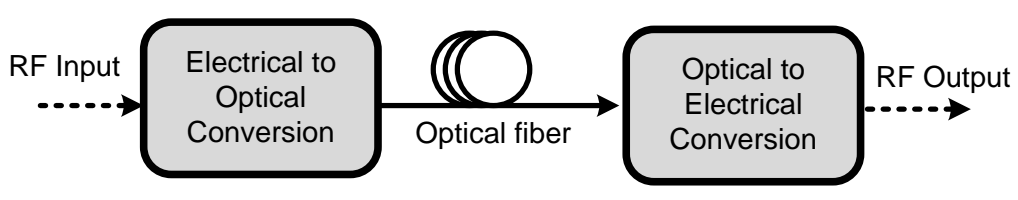

Figure 1.1: Schematic of a microwave photonic link.

bands, spectral efficient modulation formats like quadrature phase shift keying (QPSK),m-ary quadrature amplitude modulation (M-QAM), or even orthogonal frequency division multiplexing (OFDM) based modulation must be considered especially in a bi-directional link scenario.

\subsection{Why photonics?}

Generation and transmission of radio signals was previously performed using electrical technology, where the bandwidth and transmission distances were not in comparison with today's requirements. With the invention of the laser [17], and hence the birth of optical communications, the bandwidth and the distances started to increase drastically. In parallel, the use of optical communications for transmission of radio/wireless signals started to gain a lot of interest [18 21] owing to the great advantages of photonic technologies like high bandwidth, flexibility, and low transmission losses. This field of using photonic technologies for microwave applications is named as Microwave Photonics (MWP) 22 25] and its applications for transmission and distribution of radio signal is referred to as Radio-over-fibre (RoF). Some of the main applications of MWP include high performance analog links [26, 27], cellular/mobile links [28 30], RADAR, CableTV [31,32] etc. All these applications are possible because of the advantages of MWP technology like low cost, small form factor, low attenuation, higher operational frequencies, etc [24]. Another application of MWP technology is Gb/s wireless, which will be introduced in the next section.

Figure 1.1 shows the basic schematic of a MWP link. The basic components of a MWP link are the electrical to optical (E/O) and optical to electrical $(\mathrm{O} / \mathrm{E})$ converters. The $\mathrm{E} / \mathrm{O}$ converter takes a radio frequency 
(RF) signal as input, and converts it to an optical signal. This function can be either performed directly by the optical source (by direct modulation of the laser current), or by using an external electro-optical modulator in combination with the laser [18,33. Lasers with direct modulation capability were extensively investigated and several technologies for making lasers were proposed. One of the first $\mathrm{GHz}$ bandwidth lasers were based on Fabry-Perot (FP) emitting at $1300 \mathrm{~nm}$ 34, 35] and further advances pushed the bandwidth upto $40 \mathrm{GHz}$ [36]. Other technologies like Distributed Feedback (DFB) lasers were also developed for operating in the $1550 \mathrm{~nm}$ wavelengths [37, where modulation bandwidths of $>20$ $\mathrm{GHz}$ were obtained using Multi-Quantum Well techniques [38 40].

Even though directly modulated lasers are a cost-effective solution for certain applications, they are limited by the modulation bandwidth, noise figure, dynamic range, and modulation index. As an effort to increase the bandwidth of optical sources, optical modulators [41] were developed on several technologies. The most common materials used for external modulators are $\mathrm{LiNbO}_{3}$, III-V semiconductor devices, and electrooptic polymers. Of all the external modulators, $\mathrm{LiNbO}_{3}$ travelling-wave modulators have the most mature technology, which have an MachZehnder interferometric structure. One of the earlier modulators were based on Titanium diffused Lithium Niobate $\mathrm{Ti}-\mathrm{LiNbO}_{3}$,42, and in the early $90 \mathrm{siNbO}_{3}$ modulators upto bandwidth of $75 \mathrm{GHz}$ were demonstrated 43 45. The problem with earlier $\mathrm{LiNbO}_{3}$ based modulators was the high driving voltage requirements at higher bandwidths. $\mathrm{LiNbO}_{3}$ modulators today have evolved to a greater extent, where reduction in the drive voltages $\left(\mathrm{V}_{\pi}\right)$, advancements in fabrication process, reducing the modulator chirp, were achieved 46, 47, and are commercially available and installed in several optical communication systems.

The other kind of external modulator is called the electroabsorption modulator (EAM) which is based on III-V semiconductor devices. EAMs are especially attractive for MWP applications for their small size, high bandwidth, polarization insensitivity, low driving voltages, and their potential for possible integration with other optical devices like lasers or PDs. EAMs are based on electroabsorption effect which can be either the Franz-Keldysh effect found in bulk semiconductors [48] or the Quantum Confined Stark Effect (QCSE) found in quantum-well materials [4]. 
For achieving higher modulation index and lower driving voltage QCSE based devices attained a lot of research and commercial interest. Devices with driving voltages as low as $1.2 \mathrm{~V}[50$ and upto $100 \mathrm{GHz}$ of bandwidth [51] were demonstrated.

The conversion of an optical signal to electrical O/E conversion is crucial to MWP and is performed in a metal-semiconductor-metal, p-i-n, and avalanche photo-diodes (PD). The most common PDs in MWP application are the p-i-n type made with InGaAs and InGaAsP for their use in 1300 and $1550 \mathrm{~nm}$ bands. New kinds of PDs are in development for achieving higher bandwidth and output powers. The uni-travellingcarrier photo-diode (UTC-PD) [52] is one such device where bandwidths of more than $300 \mathrm{GHz}$ and high output powers of around $20 \mathrm{dBm}$ have been demonstrated [53]. Recently UTC-PD up to $1 \mathrm{THz}$ bandwidth integrated with antenna has been demonstrated 54 for THz sources applications.

\subsection{Photonics for $\mathrm{Gb} / \mathrm{s}$ wireless: State of the art}

The use of broadband services over wireless medium is on the rise, and new architectures for supporting the fourth generation mobile standards like WiMAX, LTE are being investigated [55]. Apart from the new mobile wireless standards, new wireless services for several applications like wireless HDTV, broadband fixed data services, security monitoring, etc. have opened new frequency bands like $5 \mathrm{GHz}, 60 \mathrm{GHz}$ etc . Figure 1.2 shows a schematic of several wireless applications. All the data generated by these wireless applications need an optical backbone network, which in-turn is bringing the fibre very close to the antenna, giving rise to picocellular architectures [56. In such scenarios, RoF technology offers a flexible solution, with the central station (CS) base station (BS) configuration. With these the wireless signals are optically transmitted from the CS, where all the signal generation and processing is centralised, to the BSs and radiated over air. This greatly reduces the complexity of the BSs and thus enable more BSs favouring a pico-cellular architectures by reducing the BSs cost. Another added advantage is that system updates can be easily made at the CS without changing the RF modules at the 


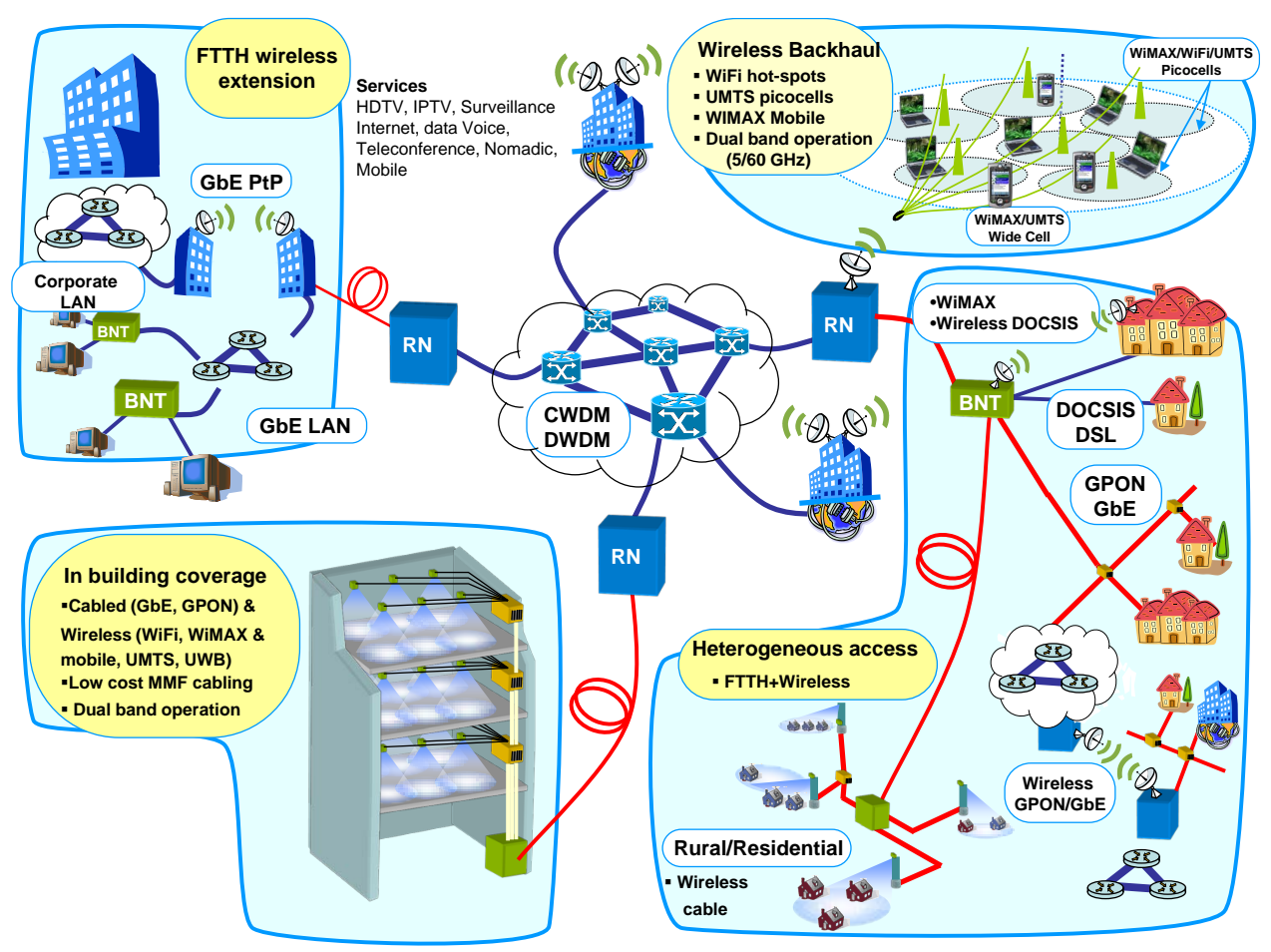

Figure 1.2: Artistic illustration of a futuristic access network with integrated wired and wireless systems.

BSs.

For multi-Gb/s wireless links in the mmW frequency bands, fibre networks plays a more crucial role in transmitting the wireless signals to the antenna BS. Since mmW signals have very narrow beam-width, and high attenuation, more and more BSs are needed to serve a relatively large geographical area. One option for providing such connectivity is to have a mmW local oscillator (LO) in each BS where the received baseband of intermediate frequency (IF) signals are up-converted to the RF frequency. This solution is attractive for example in a pico-cellular architecture for indoor wireless signal distribution [57], and at lower frequencies, but for architectures where outdoor BSs are located in remote areas, the operational cost (OPEX) will increase with complicated BSs. Another attractive solution is integrating photonics into wireless signal generation, where the wireless signals are optically generated at the CS, 
and transmitted to the remote BSs. In a broadcast architecture like FTTH, this solution can be especially interesting, where optically generated wireless signals can be sent to several BSs, which will connect several users as seen in fig. 1.2. RoF systems also offer the flexibility to be used for providing different services to different BSs using WDM 58 or sub-carrier multiplexed systems (SCM) [59]. This high level of interaction between wireless and wire-line technology will play a crucial role in futuristic flexible access network architectures.

\subsubsection{Intensity modulated/direct detection (IM/DD) based wireless links}

Use of MWP for broadband wireless started during late 90s 60 65 mainly to overcome the limitations of electrical technology. These early systems were designed to directly process baseband signal optically and up-convert to the desired RF frequencies, which otherwise electrically would require high speed I/Q processing, multi-stage conversion, and high speed digital to analog converters. The first method was based on generating spectral efficient vectorial modulation formats using a technique named as Microwave Photonic Vector Modulation. In this technique baseband data is modulated and up-converted directly using photonic technique resulting in a M-ary quadrature amplitude/phase shift keyed modulation (M-QAM/PSK) 60,62,64].

During the same time, Hirata et al. proposed a simple amplitude shift keyed (ASK) modulation format based $\mathrm{mmW}$ wireless generation and demodulation 63. This technique is based on directly up-converting a baseband optical ASK signal to the desired carrier frequency. Since ASK is a bandwidth inefficient modulation format, the frequency was chosen to be around $120 \mathrm{GHz}$ and upto $10 \mathrm{~Gb} / \mathrm{s}$ wireless link was experimentally demonstrated 65]. ASK links have gained some interest for $10 \mathrm{~Gb} / \mathrm{s}$ wireless links, where Weiss et al., have demonstrated a $12.5 \mathrm{~Gb} / \mathrm{s}$ ASK wireless link in the $60 \mathrm{GHz}$ band [66. The downside of this technique is the total occupied bandwidth is higher than the $7 \mathrm{GHz}$ allowed in the 60 GHz. Similarly, Ng'Oma et al., have also demonstrated an ASK wireless link in the $60 \mathrm{GHz}$ band, but by using tight RF single side-band (SSB) filtering and linear equalization techniques, which enables doubling the 


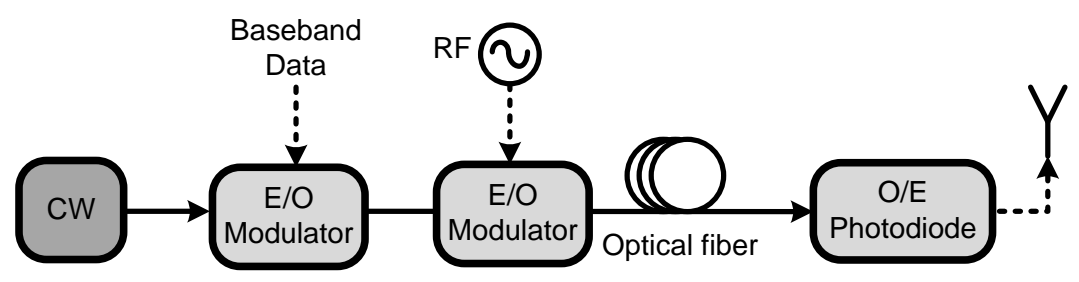

(a)

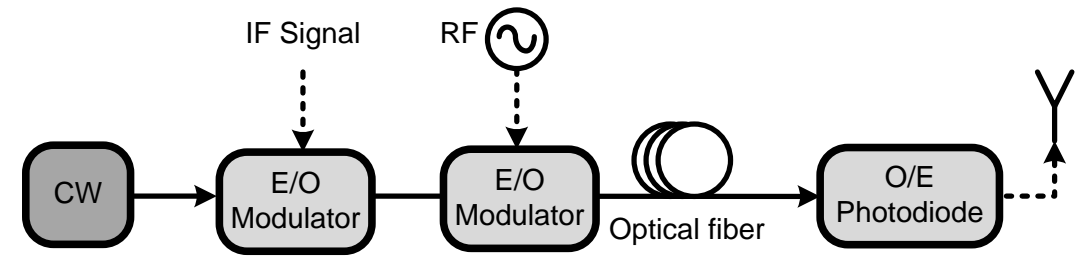

(b)

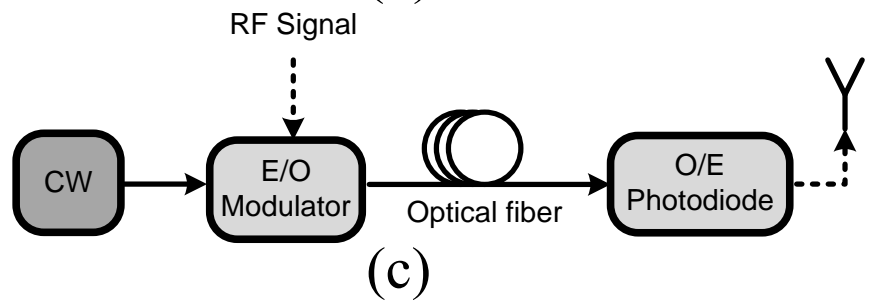

Figure 1.3: Schematics of several photonic techniques used for generation and transmission of $\mathrm{Gb} / \mathrm{s}$ wireless signals.

bit-rate 67.

The photonics based wireless systems have taken a new phase, especially in the $60 \mathrm{GHz}$ band, with the recent developments in electric technology like SiGe [68, 69], where the optics is used to modulate the RF signals and transmit over fibre. These techniques are based on electrically generating a very high bit-rate wireless signal either in IF or RF frequency, and modulating on an optical carrier and transmitting to the antenna BS. The first experimental demonstration using such a concept was a 16 $\mathrm{Gb} / \mathrm{s}$ wireless link in the $24 \mathrm{GHz}$ band [70], where an electrical orthogonal frequency division multiplexed (OFDM) QPSK signal was modulated using a single side-band configuration (SSB) on an optical carrier. Similarly, using a SSB configuration and frequency quadrupling, a $28 \mathrm{~Gb} / \mathrm{s}$ 
wireless link in the $60 \mathrm{GHz}$ band with up to $100 \mathrm{~km}$ of fibre transmission was demonstrated by Lin et al 71]. These SSB configurations have the advantage of robustness to fibre dispersion, but involve a complex method for generation containing one or more MZMs.

Other simpler generation techniques have been demonstrated based on simple double side-band modulation (DSB) without the need of complex modulators. Weiss et al [72] have demonstrated a $27 \mathrm{~Gb} / \mathrm{s}$ wireless link by simply modulating an IF signal on an optical carrier, and later converting it to RF. Ng'Oma et al., [73 have also demonstrated a $21 \mathrm{~Gb} / \mathrm{s}$ wireless link, which is simply modulating a $60 \mathrm{GHz} \mathrm{RF}$ signal on an optical carrier and transmitting to the antenna site. Figure 1.3 summarizes the most common techniques used for wireless signal generation/transmission.

\subsubsection{Optical heterodyne techniques}

Optical heterodyne mixing is a process where two optical carriers are mixed in a photo-diode to generate $\mathrm{mmW}$ carriers and potentially can generate any frequency theoretically limited by the photo-diode bandwidth. The advantage of optical heterodyne compared to IM/DD techniques for RF signal generation is that the optical heterodyne technique generates higher RF power, since all the optical power contributes to the $\mathrm{RF}$ signal generation. Wireless signal generation based on heterodyne mixing of two optical sources suffer from phase and frequency offsets of the optical carriers, which will severely limit the performance of the RF signal. Other than the offsets of the optical carriers, the linewidth of the optical signals determine the total phase noise of the RF signal. To counter these effects, phase control mechanisms such as optical injection locking (OIL) [74 and optical phase locked loops (OPLL) [75] techniques are proposed. The OIL technique generates low phase noise signals with lower locking range, whereas OPLLs have higher locking range but require lower loop delays. OPLL based wireless signal generation has been performed using an integrated device contained two OPLLs [76] A combination of both these techniques called optical injection phase locked loops (OIPLL) [77] offer both low phase noise signal generation and greater locking range. Wireless signal generation based on OIPLL has also been demonstrated [77] using two standard DFB lasers. 


\subsection{Outline of the thesis}

Most of the existing techniques for generation of wireless signals use electrical signal generators for the generation of an intermediate frequency signal, which is then converted to RF frequencies using several RoF upconversion techniques. The main limitations is the limited speed of the available digital to analogue converters used in the electrical signal generators. Also the fibre's chromatic dispersion induced power fading limits to a great extent the transmission distance. The change in modulation format between the optical and wireless signals does not allow seamless integration of wireless services in an optical network creating a new bottle neck.

The aim of this thesis is to provide generation and demodulation techniques using pure baseband signals, which would allow easy integration of wireless and optical communication systems. Using baseband signal processing and common RoF techniques, advanced modulation formats like MQAM/MPSK are generated and demodulated, while keeping all the processing centralised and making the antenna BS as simple as possible. Also a certain part of the thesis is dedicated towards direct generation of wireless signals with the same optical modulation format, which will provide totally transparent wireless/wired services seamless integration.

The introductory chapter was aimed at giving a basic information on MWP and its several applications. Chapter 2 contains an overview of several RoF techniques used in generation and demodulation of wireless signals, and thus understanding the performance of several components in RoF applications. The work performed in this thesis related to photonic vector modulation and demodulation is described in chapter 3 . In chapter 4 the use of baseband optical modulation and demodulation techniques for multi-Gb/s wireless links is discussed, where direct up-conversion of optical baseband data to RF frequencies is presented. Chapter 5 concludes the thesis with an outlook into future. 
CHAPTER 2

\section{Introduction to \\ Radio-Over-Fibre Techniques}

In this chapter, the basic techniques of up-conversion and down-conversion of an ROF link are described, which will form the background for the forthcoming chapters. The various devices that are crucial in a RoF link are identified and their parameters that influence the performance of a link are studied in section 2.1. The use of optical modulators in a RoF downlink which perform the up-conversion to RF frequency is described in section 2.2. Similarly, the use of modulators in RoF uplinks are described in section 2.3 .

\section{$2.1 \quad$ Optical Devices}

A radio-over-fibre link transmits radio signals from the CS to BS and vice versa using an optical fibre. This thesis deals with the optical generation of multi-Gb/s wireless signals, which is performed at the CS. The generation of wireless signals consists of two main blocks: the data 
modulation of an optical source and later up-conversion to the desired $\mathrm{RF}$ carrier frequency. In a simple configuration, the optical source like a semiconductor laser can also be used for data modulation by simply modulating the current of the laser also named as directly modulated lasers (DML). For the RF up-conversion an external modulator is used to modulate the optical signal with a local-oscillator. This is typically performed using external modulators like MZM or EAM. The optically generated wireless signals are transmitted over an optical single mode fibre (SMF) ${ }^{1}$ to the antenna BS, where the optical signal is converted to electrical signal in a photo-diode prior to radiating over air. In the uplink, the wireless signals received at the antenna in a BS are modulated on an optical carrier using an external modulator, and transmitted to the CS using a SMF to perform the demodulation. In conclusion, the crucial components that build a RoF link are the optical source, the external modulators and the photo-diodes. In the following sections, the parameters of each of these devices that influence the performance of an RoF link are described.

\subsubsection{Directly modulated laser}

The ability of directly modulating a laser is an attractive cost-effective solution for RoF links. DMLs were extensively studied for their use in optical communication systems for metro applications [78,79. The limiting factors of a DML are its modulation bandwidth, extinction ratio, chirp and linearity, but for baseband data modulation these parameters do not greatly affect the performance in a typical RoF link. The output power of a DML can be written as:

$$
P_{\text {out }}=\frac{\eta_{d}}{2}\left(I_{b}+I_{m}-I_{t h}\right) \frac{h \nu}{e}
$$

Where $\eta_{d}$ is the laser differential quantum efficiency $I_{b}, I_{m}$ and $I_{t h}$ are the bias, modulation and threshold currents. The optical field of the laser may be written as:

$$
E(t)=\sqrt{2 P_{\text {out }}} \exp j(\omega t)
$$

\footnotetext{
${ }^{1}$ In this work, only standard single mode fibre transmission is considered.
} 


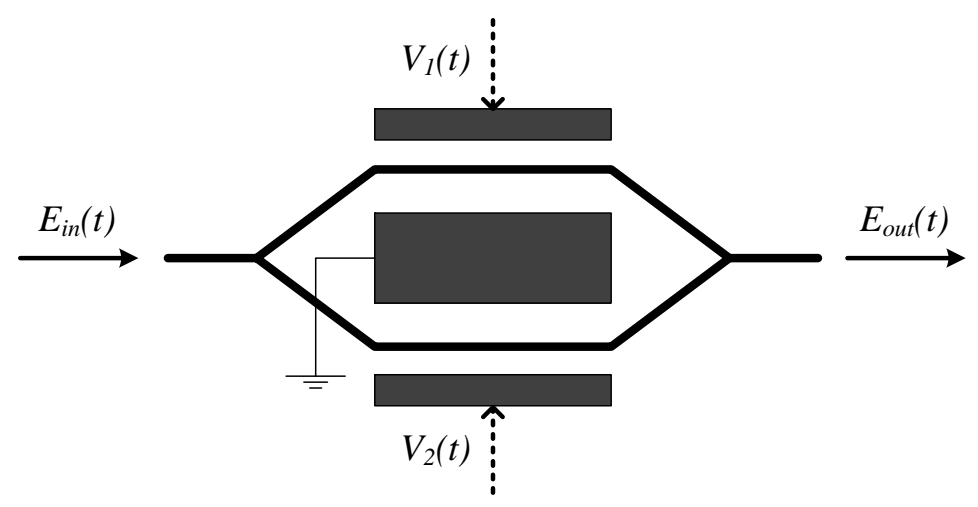

Figure 2.1: Schematic of a typical dual-drive Mach-Zehnder modulator

In directly modulated semiconductor lasers when the carrier density is changed due to the changes in the modulation current, changes the refractive index in the active area resulting in a frequency deviation also called as frequency chirp [80]. The effect of chirp in an optical communication system is spectral broadening due to dispersion in a SMF, which limits the total transmission distance 81]. The frequency deviation $\delta \vartheta$ and the phase shift $\phi$ induced by the chirp can be expressed as 82 :

$$
\begin{aligned}
\Delta \vartheta(t) & =\frac{\alpha_{L}}{4 \pi}\left[\frac{1}{P_{\text {out }}} \frac{d P_{\text {out }}}{d t}+\kappa P_{\text {out }}\right] \\
\phi(t) & =-\frac{\alpha_{L}}{2}\left[\ln \left(P_{\text {out }}\right)+\kappa P_{\text {out }}\right]
\end{aligned}
$$

Where $\alpha_{L}$ is the Henry's linewidth enhancement factor and $\kappa$ the adiabatic chirp coefficient. From eqn. 2.2 and eqn. 2.4, the total optical field can be written as:

$$
E(t)=P_{\text {out }}^{\frac{1-j \alpha_{L}}{2}} \exp j\left(\omega t-\theta_{L}\right)
$$

where, $\theta_{L}=\frac{\alpha_{L} \kappa}{2} P_{\text {out }}$.

\subsubsection{Mach-Zehnder Modulator}

The most common modulator used in RoF systems is the Mach-Zehnder modulator, which is based on a Mach-Zehnder interferometric structure. 
In a MZM an electrical voltage induces phase shift between the two arms of the interferometer and depending upon the quantity of phase shift, constructive or destructive interference occurs. This interference results in intensity modulation of the optical signal by the electrical voltage applied. Figure 2.1 shows the schematic of a MZM. The electrical voltage induced optical phase shift can be expressed as:

$$
\phi(t)=\eta_{M Z} V(t), \quad \eta_{M Z}=\frac{\pi}{V_{\pi}}
$$

where $V(t)$ is the applied voltage, and $V_{\pi}$ is the voltage required to induce a $\pi$ phase shift. The parameter $V_{\pi}$ is an intrinsic property of the modulator which depends on various design parameter and the material used. Considering an asymmetric MZ modulator with a finite extinction ratio $\epsilon$, the optical field at the output of the MZM can be written as:

$$
\begin{aligned}
E_{\text {out }}(t)= & \frac{E_{\text {in }} \exp j(\omega t)}{2}\left(\exp j\left(\phi_{1}(t)\right)+\gamma \exp j\left(\phi_{2}(t)\right)\right) \\
= & \sqrt{\frac{P_{\text {in }} T_{f f}}{2}} \sqrt{1+\gamma^{2}+2 \gamma \cos \left(\eta_{M Z}\left[V_{1}(t)-V_{2}(t)\right]\right)} \\
& \cdot \exp j\left(\omega t+\theta_{M Z}(t)\right)
\end{aligned}
$$

where $\gamma$ the parameter related to the MZM extinction ratio is expressed as 83$](\sqrt{\epsilon}-1) /(\sqrt{\epsilon}+1)$. The transfer function of the MZM according to eqn. 2.7 is plotted in fig. 2.2 for different extinction ratios.

$\theta_{M Z}(t)$ in eqn. 2.7 is the instantaneous phase shift varying with the applied voltage, which is expressed as:

$$
\theta_{M Z}(t)=\tan ^{-1}\left[\frac{\sin \left(\phi_{1}\right)+\gamma \sin \left(\phi_{2}\right)}{\cos \left(\phi_{1}\right)+\gamma \cos \left(\phi_{2}\right)}\right]
$$

For $\gamma \approx 1$, i.e extinction ratio $>25 \mathrm{~dB}$, the eqn. 2.7 can be simplified to:

$$
\begin{aligned}
E_{\text {out }}(t)= & \sqrt{\frac{P_{\text {in }} T_{f f}}{2}}\left[1-\gamma+2 \sqrt{\gamma} \cos \left(\frac{\eta_{M Z}}{2}\left[V_{1}(t)-V_{2}(t)\right]\right)\right] \\
& \cdot \exp j\left(\omega t+\theta_{M Z}(t)\right)
\end{aligned}
$$

The chirp parameter $\left(\alpha_{M Z}\right)$ is defined as the ratio of the phase modulation to amplitude modulation [80]. Applying small signal modulation 


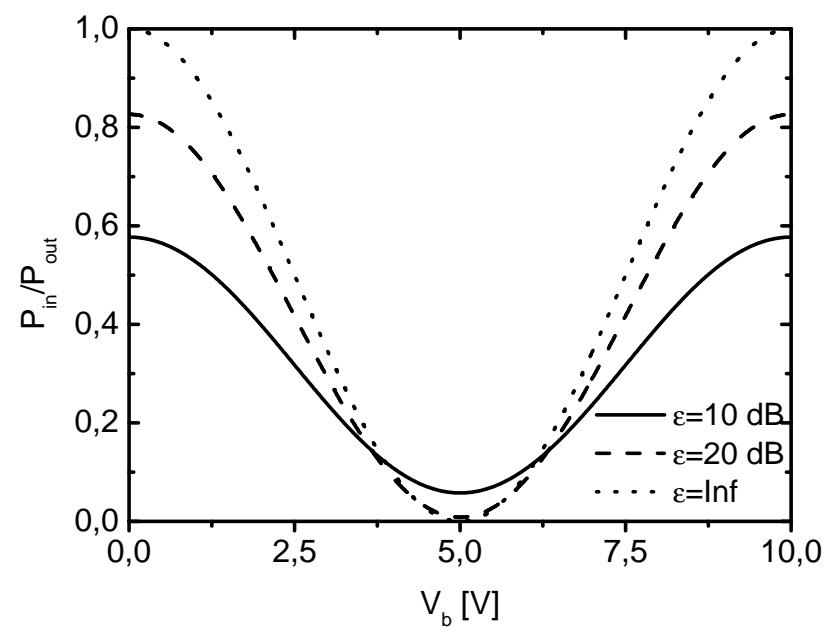

Figure 2.2: The transfer function of a MZM at different extinction ratios.

and bias voltage $\left(V_{b}\right)$ to the MZM, and from eqn. 2.6 and 2.9 the chirp parameter is expressed as:

$$
\alpha_{M Z}=P_{\text {out }} \frac{\frac{d \theta_{M Z}}{d t}}{\frac{d P_{\text {out }}}{d t}}=\frac{\bar{V}_{1}+\gamma^{2} \bar{V}_{2}+\gamma\left(\bar{V}_{1}+\bar{V}_{2}\right) \cos \left(\frac{\pi \bar{V}_{b}}{\bar{V}_{\pi}}\right)}{\gamma\left(\bar{V}_{1}-\bar{V}_{2}\right) \sin \left(\frac{\pi \bar{V}_{b}}{\bar{V}_{\pi}}\right)}
$$

where $\bar{V}$ is the peak voltage of the RF signal $V(t)$.

From eqn.2.10 it may be observed that in ideal push-pull modulation, the finite extinction ratio creates a residual chirp. The advantage of using a MZM is that the chirp can be compensated by [84] creating an imbalance between the two driving voltages. Thus for a zero chirp modulation, the relation between the driving voltages is:

$$
\begin{aligned}
\bar{V}_{1} & =-\bar{V}_{2} \varphi\left(V_{b}\right) \\
\varphi\left(V_{b}\right) & =\frac{\gamma\left(\gamma+\cos \left(\frac{\pi V_{b}}{V_{\pi}}\right)\right)}{1+\gamma \cos \left(\frac{\pi V_{b}}{V_{\pi}}\right)}
\end{aligned}
$$

The relation between the driving voltages depend on the bias point of the MZM, and for intensity modulation using quadrature bias point, the relation between the voltages is $V_{1}=-\gamma^{2} V_{2}$ [84. For a $100 \%$ extinction 
ratio $(\gamma=1)$, the output optical field of a MZM is:

$$
E_{\text {out }}(t)=\sqrt{2 P_{\text {in }} T_{f f}} \cos \left[\frac{\pi}{V_{\pi}}\left(\frac{V_{1}(t)-V_{2}(t)}{2}\right)\right] \exp j\left(\omega t+\frac{\pi}{V_{\pi}}\left(\frac{V_{1}(t)+V_{2}(t)}{2}\right)\right)
$$

\subsubsection{Electroabsorption modulator}

Electroabsorption modulators are another kind of optical external modulators which perform intensity modulation by changing the absorption in the material depending on the electrical field applied. As discussed in previous chapter, EAMs are advantageous in several application because of their polarization insensitivity, and low driving voltages, and their potential possibility of integration with other optical devices like lasers and PDs. On the other hand EAMs suffer from huge insertion losses and bias voltage drifts. EAMs have received a lot of interest for its use in both baseband digital communications [85, 86] and RoF systems [87, 88]. The combination of EAMs huge bandwidth and low driving voltages makes them interesting for analogue applications.

The absorption transfer function of an EAM can be modelled as 89

$$
T(V)=\frac{P_{\text {out }}}{P_{\text {in }}}=\exp \left(-k L\left(V-V_{0}\right)^{c}\right)
$$

Where $k, V_{0}$ and $c$ are the EAMs physical parameters which are chosen as $k=3 \times 10^{3}, c=1.5$ and $V_{0}=0.35 \mathrm{~V} . L$ is the length of the EAM section which is assumed to be $160 \mu \mathrm{m}$. Figure 2.3 shows the transfer function of an EAM according to the eqn. 2.13 and from the measured data. Similar to a MZM, EAM also has frequency chirping according to [80] and is expressed as:

$$
\frac{d \phi}{d t}=\frac{\alpha_{E}}{2 P_{\text {out }}} \cdot \frac{d P_{\text {out }}}{d t}
$$

Thus, the output field of an EAM can be written as:

$$
E_{\text {out }}=\sqrt{2 P_{\text {in }}} L_{E A M} T(V)^{\frac{1+j \alpha_{E}}{2}} \exp j\left(\omega t+\frac{\alpha_{E}}{2} \ln P_{\text {in }}\right)
$$

Where $\alpha_{E}$ is the chirp parameter of the EAM which depends on the bias voltage. $L_{E A M}$ is the EAM insertion losses. 


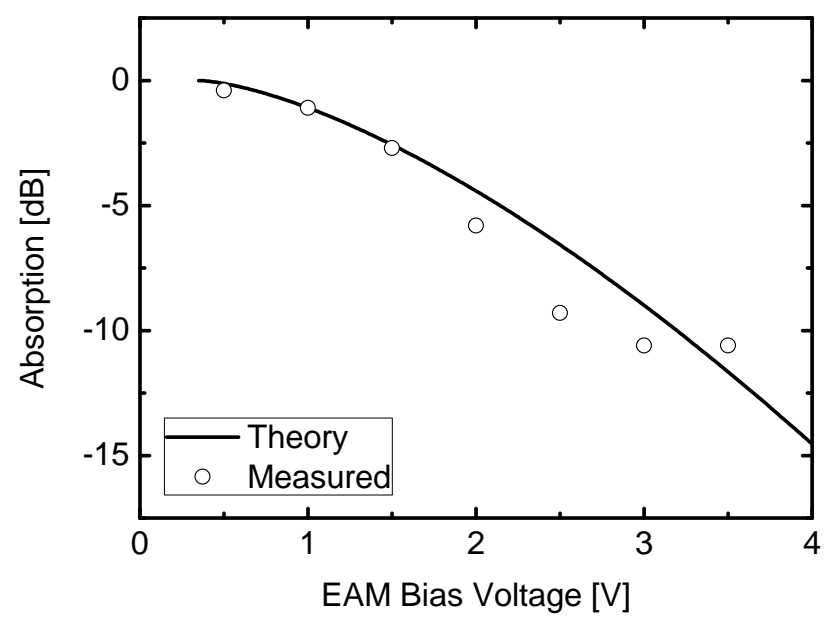

Figure 2.3: The theoretical and measured absorption transfer function of an EAM.

\subsubsection{Optical Fibre}

In current telecommunication networks optical fibre is the main medium of communication. In RoF links, both single mode and multi-mode fibres are used depending on the application, but in this thesis only SMF transmission is considered. The main advantage of optical fibre is its low loss, but there are several limiting factors in optical fibre transmission like chromatic dispersion, polarization mode dispersion, and fibre non-linearities. Chromatic dispersion is one of the crucial parameters for RoF system design. In an optical fibre due to its intrinsic material properties, some spectral components of the signal travel slower with respect to the other, and the time delay between the spectral components results in broadening of the spectrum for a broadband signal, or phase shift between spectral components in a multi-tone signal. The frequency response of an optical fibre can be expressed as:

$$
H_{f i b}(\omega)=\left|H_{f i b}(z)\right| \exp j(\beta(\omega) z)
$$

Where $\left|H_{f i b}(z)\right|=L_{f i b}$ is the loss of the fibre depending on the distance $z=L$. $\beta$ is the propagation constant evaluated defined as 90 :

$$
\beta(\omega) \approx \beta_{0}+\beta_{1}(\Delta \omega)+\frac{1}{2} \beta_{2} L(\Delta \omega)^{2}+\cdots
$$


Where $\beta_{0}$ results in a constant phase shift, and $\beta_{1}$ in constant delay which depends on the frequency difference $\Delta \omega=\omega-\omega_{0}$. The second derivative of the propagation constant also known as the group velocity parameter which contributes to chromatic dispersion is expressed as:

$$
\beta_{2}=-D \frac{\lambda_{0}^{2}}{2 \pi c}
$$

Where $\mathrm{D}$ is the dispersion parameter measured in $\mathrm{ps} / \mathrm{nm}-\mathrm{km}$. The time delay $(\Delta T)$ introduced between the spectral $\left(\Delta \lambda=\lambda-\lambda_{0}\right)$ components is:

$$
\Delta T=D L \Delta \lambda
$$

\subsubsection{Noise Sources}

In a IM/DD RoF link, the total noise generated by various components in the system limit the performance of the system and determine the total signal to noise ratio (SNR). In a RoF link without optical amplification, the main sources of noise are the laser, photo-diode and the electrical amplifier used prior to the antenna. In lasers the relative intensity noise (RIN) is mainly caused by fluctuations in the internal cavity and the RIN coefficient is measured in $\mathrm{dB} / \mathrm{Hz}$. The RIN increases with the optical power, and in systems with high optical powers, the total SNR is RIN limited. The noise variance from the laser RIN is defined as:

$$
\sigma_{R I N}^{2}=\mathfrak{R}^{2} P_{i n}^{2} R I N \Delta f
$$

Where $\mathfrak{R}$ is the photo-diode responsivity, $P_{\text {in }}$ the average optical power input to the photo-diode and $\Delta f$ the bandwidth of the signal. When the optical power is too low, the system is limited by the photo-diode shot noise, whose variance is defined as:

$$
\sigma_{\text {shot }}^{2}=2 q\left(\Re P_{\text {in }}+i_{\text {dark }}\right) \Delta f
$$

Where $q$ is the charge of electron and $i_{\text {dark }}$ the photo-diode dark current. Another important source of noise is the thermal noise of the electrical amplifiers in a RoF link. The variance of the thermal noise is expressed as:

$$
\sigma_{\text {thermal }}^{2}=\frac{4 k T \Delta f}{R_{L}} F
$$


Where $k$ is the Boltzmann's constant, $T$ is the temperature in Kelvin, and $R_{L}$ is the load resistance. The effect of all these noise contributions on a typical RoF link will be analysed in the following section.

\subsection{RF Up-conversion Techniques}

$\mathrm{RF}$ up-conversion is the process of converting an optical baseband or IF signal to the RF frequency. This is the most crucial process for wireless signal generation in a RoF link, where an optical baseband signal is optically mixed with a high frequency LO signal in an external modulator. Figure 2.4 shows the schematic of a RoF link with wireless signal generation using DML for data modulation and external modulator for $\mathrm{RF}$ up-conversion. In this system the baseband data directly modulates the current of the laser and subsequently the optical output of the laser. Later a LO signal at frequency $f_{L O}$ is modulated using an external modulator, which results in up-conversion of the baseband signal to the LO frequency. Both MZM and EAM can be used to perform RF up-conversion, which is described in the following sections.

\subsubsection{Up-conversion using Mach-Zehnder modulator}

Mach-Zehnder modulator as described in the previous sections is used for RF up-conversion, and the non-linear transfer function of the MZM enables generation of higher order harmonics by creating multiples of

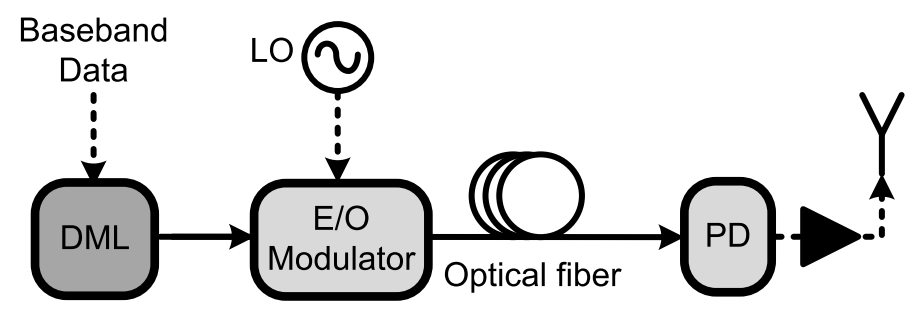

Figure 2.4: Schematic of a RoF up-converting link. 
the LO frequency [91, 92]. However, the non-linear transfer function also induces harmonic distortion which is discussed in the following sections.

The electrical signals applied to the arms of the MZM can be described as:

$$
\begin{aligned}
& V_{1}(t)=\varphi \frac{V_{m}}{2} \sin \left(\omega_{L O} t\right)+V_{b} \\
& V_{2}(t)=-\frac{V_{m}}{2} \sin \left(\omega_{L O} t\right)
\end{aligned}
$$

Considering a RoF link as shown in fig. 2.4 and applying the above electrical signals (eqn. 2.23) to an optical signal as described in eqn. 2.5 using a MZM (according to eqn. 2.9p), the output of the MZM is:

$$
\begin{aligned}
E_{\text {out }}= & P_{\text {in }}(t)^{\frac{1-j \alpha_{L}}{2}} \sqrt{\frac{T_{f f}}{2}}(1-\gamma) \exp j\left(\omega t-\theta_{L}(t)\right) \\
& +P_{\text {in }}(t)^{\frac{1-j \alpha_{L}}{2}} \sqrt{2 \gamma T_{f f}} \cos \left[\frac{\eta_{M Z}}{2}\left(\frac{V_{m}(\varphi+1)}{2} \sin \left(\omega_{L O} t\right)+V_{b}\right)\right] \\
& \cdot \exp j\left(\omega t-\theta_{L}(t)+\theta_{M Z}(t)\right)
\end{aligned}
$$

The above equation contains two terms, where the first term is an unmodulated optical signal due to the finite extinction ratio of the MZM, whereas the second term contains the LO signal modulation on it. Expanding the second term using Bessel's function [93], the output of the MZM with an LO signal modulation can be written as:

$$
\begin{aligned}
E_{\text {out }}= & P_{\text {in }}(t)^{\frac{1-j \alpha_{L}}{2}} \sqrt{\frac{T_{f f}}{2}}(1-\gamma) \exp j\left(\omega t-\theta_{L}(t)\right) \\
& +P_{\text {in }}(t)^{\frac{1-j \alpha_{L}}{2}} \sqrt{2 \gamma T_{f f}} \sum_{n=-\infty}^{\infty} J_{n}\left(m_{L O}\right) \cos \left(\frac{\pi V_{b}}{2 V_{\pi}}-\frac{n \pi}{2}\right) . \\
& \exp j\left(\left(\omega+n \omega_{L O}\right) t\right) \exp j\left(-\theta_{L}(t)+\theta_{M Z}(t)\right)
\end{aligned}
$$

Where $m_{L O}$ is the modulation index, which is expressed as:

$$
m_{L O}=\frac{\pi V_{m}(1+\varphi)}{2 V_{\pi}}
$$

For up-converting the optical signal to the RF frequency $f_{L O}$ using a LO signal of frequency $f_{L O}$, the modulator should be biased at the quadrature 
point (QB) $V_{b}=V_{\pi} / 2$, which results in a double side-band RF modulation (DSB). The directly modulated laser is modulated with a bi-polar $\left(I_{m}(t)=-B, B\right)$ signal of non-return-to-zero (NRZ) to drive the laser to two power levels above threshold $P_{O F F}$ and $P_{O N}$, which satisfy the following conditions:

$$
\begin{array}{r}
P_{O N}-P_{O F F}=2 \eta B \\
P=\frac{P_{O N}+P_{O F F}}{2}
\end{array}
$$

Where $P$ is the laser average power. In such a case, the output of the MZM is:

$$
\begin{aligned}
E_{\text {out }, Q B}= & {[P+\eta I(t)]^{\frac{1-j \alpha_{L}}{2}} \sqrt{\gamma T_{f f}}\left[\left(\frac{\sqrt{2}(1-\gamma)}{\sqrt{\gamma}}+J_{0}\left(m_{L O}\right)\right) \exp j(\omega t)\right.} \\
& +\left\{J_{1}\left(m_{L O}\right) \exp j\left(\left(\omega \pm \omega_{L O}\right) t\right)+J_{2}\left(m_{L O}\right) \exp j\left(\left(\omega \pm 2 \omega_{L O}\right) t\right)\right. \\
& \left.\cdots\} \exp j\left(\theta_{M Z}(t)\right)\right] \exp j\left(-\theta_{L}(t)\right)
\end{aligned}
$$

Similarly, for up-conversion by frequency doubling using a LO signal at half the frequency $\left(\omega_{L O} / 2\right)$, the MZM should be biased at minimum transmission point (MiTB) $\left(V_{b}=V_{\pi}\right)$. When the MZM is biased in the minimum transmission point, a double side-band suppressed carrier (DSB-SC) modulation is generated by the second harmonics of the LO signal at frequency $\omega_{L O} / 2$. The output of the MZM for MiTB is:

$$
\begin{aligned}
E_{\text {out }, M i T B}= & {[P+\eta I(t)]^{\frac{1-j \alpha_{L}}{2}} \sqrt{2 \gamma T_{f f}}\left[\frac{(1-\gamma)}{2 \sqrt{\gamma}} \exp j(\omega t)\right.} \\
& +\left\{J_{1}\left(m_{L O}\right) \exp j\left(\left(\omega \pm \frac{\omega_{L O}}{2}\right) t\right)+J_{3}\left(m_{L O}\right) \exp j\left(\left(\omega \pm 3 \frac{\omega_{L O}}{2}\right) t\right)\right. \\
& \left.\ldots\} \exp j\left(\theta_{M Z}(t)\right)\right] \exp j\left(-\theta_{L}(t)\right)
\end{aligned}
$$

From eqn. 2.28 it can be noticed that the optical field has the main carrier components dominated by the factor $J_{0}\left(m_{L O}\right)$ and the first order sidebands separated by the LO frequency $\omega_{L O}$ and so on. Similarly for the MiTB case, the even order components disappear resulting in frequency doubling as observed from eqn. 2.29 .

The RF photo-current (at QB) of the signal expressed in eqn. 2.28 after 
passing through an SMF of length $L$ can be written as:

$$
\begin{aligned}
i_{R F, Q B}(t)= & \sqrt{2 \gamma}[P+\eta I(t)] \Re T_{f f} L_{f i b}\left(1-\gamma+\sqrt{2 \gamma} J_{0}\left(m_{L O}\right)\right) J_{1}\left(m_{L O}\right) \\
& \cdot \cos \left(\omega_{L O} t\right) \cos \left(\frac{\beta_{2} L}{2}\left(\omega_{L O}\right)^{2}+\theta_{M Z}\right)
\end{aligned}
$$

The RF photo-current of a DSB-SC optical signal is expressed as:

$$
i_{R F, M i T B}(t)=2[P+\eta I(t)] \Re \gamma T_{f f} L_{f i b} J_{1}^{2}\left(m_{L O}\right) \cos \left(\omega_{L O} t+\beta_{2} L\left(\frac{\omega_{L O}^{2}}{4}\right)\right)
$$

From the eqn. 2.31 it can be observed that frequency doubling is achieved when the MZM is biased in the minimum transmission point to generate DSB-SC RF modulation. The fibre's dispersion induced RF power fading is seen in the eqn. 2.30, which affects the DSB modulation. But the dispersion power fading can be mitigated to a certain extent by tuning the chirp of the MZM [94]. On the other hand for MiTB with DSB-SC modulation, no RF power fading occurs, but a time delay introduced between the baseband components on the side-bands due to dispersion affect the quality of the data and is directly proportional to the product of the RF carrier frequency and length. For example, if the laser were to be driven with intermediate frequency signals instead of baseband, the power penalty induced due to dispersion for DSB-SC modulation is described in ref. [95]. The RF signal for both the QB and MiTB have two components: an unmodulated $\mathrm{LO}$ component and the data component. The unmodulated LO component is due to the intensity modulation nature of the signal generation. The unmodulated LO component is undesirable in practical situations because it saturates the power amplifiers used after the photo-diode which will limit the total wireless transmission distance. The ratio between the powers of the $\mathrm{LO}$ and data components is:

$$
\frac{P_{L O}}{P_{\text {data }}}=\frac{P^{2}}{\eta^{2} B^{2}}=\left(\frac{I_{b}-I_{t h}}{B}\right)^{2}
$$

The unwanted LO peak depends on the data modulation index, and the fig. 2.5 shows the dependence of the ratio between LO and data powers on the modulation current $(B)$. For calculating the ratio of powers, the following conditions are assumed: $I_{t h}=5 m A, B=50 \mathrm{~mA}$. For a lower LO to data RF power ratio, the data modulation index should be higher, which is achieved by modulating the laser as close to threshold 


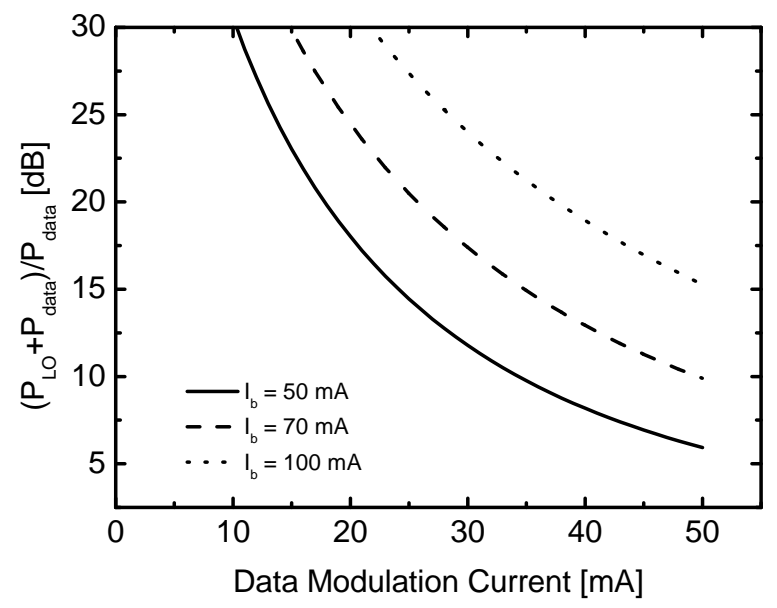

Figure 2.5: The unwanted LO peak power ratio plotted against the laser data modulation current for various bias currents.

level as possible. The minimum ratio between the LO peak and the data is $3 \mathrm{~dB}$, when $100 \%$ data modulation index in implemented $\left(I_{b} \approx I_{m}\right)$. From fig. 2.5 it can be observed that for higher bias current for a given modulation current (lower modulation index), the ratio of LO to data power increases.

To analyse the noise generated by the up-conversion process, the DC current generated at the output of the photo-diode must be calculated. The DC photo-current $i_{D C}$ for both QB and MiTB case are:

$$
\begin{aligned}
i_{D C, Q B} & =\frac{P \mathfrak{R} T_{f f} L_{f i b}}{4}\left[\left[\left(1-\gamma+\sqrt{2 \gamma} J_{0}\left(m_{L O}\right)\right]^{2}+4 \gamma J_{1}^{2}\left(m_{L O}\right)\right]\right. \\
i_{D C, M i T B} & =\frac{P \Re T_{f f} L_{f i b}}{4}\left[(1-\gamma)^{2}+8 \gamma J_{1}^{2}\left(m_{L O}\right)\right]
\end{aligned}
$$

It can be observed that the DC current is directly related to the laser's bias current. The signal to noise ratio of the up-converted signal is:

$$
\operatorname{SNR}_{Q B}=\frac{\left(\sqrt{2 \gamma} \eta B \Re T_{f f} L_{f i b}\left(1-\gamma+\sqrt{2 \gamma} J_{0}\left(m_{L O}\right)\right) J_{1}\left(m_{L O}\right)\right)^{2} \cos ^{2}\left(\frac{\beta_{2} L}{2}\left(\omega_{L O}\right)^{2}+\theta_{M Z}\right) R_{L}}{i_{D C, Q B}^{2} R I N \Delta f+2 q\left(i_{D C, Q B}+i_{d a r k}\right) \Delta f+\frac{4 k T \Delta f}{R_{L}} F}
$$




$$
\mathrm{SNR}_{M i T B}=\frac{\left(2 \eta B \Re \gamma T_{f f} L_{f i b} J_{1}^{2}\left(m_{L O}\right)\right)^{2} R_{L}}{i_{D C, M i T B}^{2} R I N \Delta f+2 q\left(i_{D C, M i T B}+i_{\text {dark }}\right) \Delta f+\frac{4 k T \Delta f}{R_{L}} F}(2
$$

From the eqn. 2.34, the SNR is calculated by varying the LO modulation and data power and plotted in fig. 2.6. From fig. 2.6(a) it can

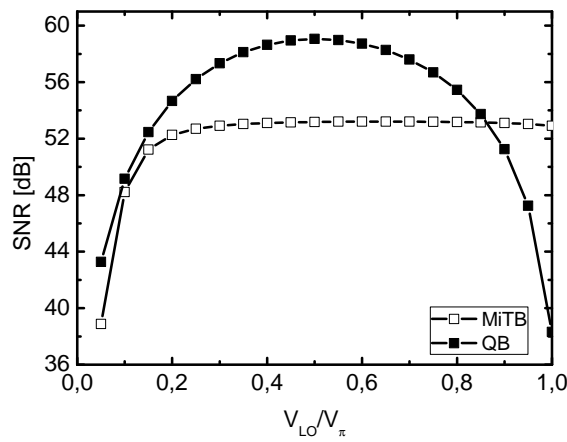

(a)

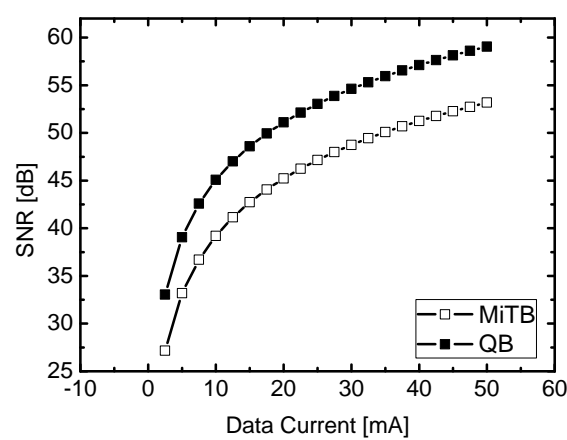

(b)

Figure 2.6: The SNR of the up-converted signals plotted against the modulation index (a), and the laser modulation data current (b).

be concluded that for $\mathrm{QB}$ condition, the ratio between the LO voltage and the modulator $V_{\pi}$ should be around 0.5. From fig. 2.6(b) it can be noticed that higher baseband data extinction ratio increase the SNR of the up-converted signal, as long as data modulation does not induce non-linearities. One main observation that can be made that for MiTB condition, in optimum modulation index, the SNR of the signal generated is around $5 \mathrm{~dB}$ lower than $\mathrm{QB}$ even though the $\mathrm{DC}$ photo current generated from the QB is higher than the MiTB case. MiTB has the advantage of frequency doubling but has poor SNR compared to the QB condition.

\subsubsection{Up-conversion Using Electroabsorption Modulator}

RF up-conversion using EAMs have attracted a lot of interest recently due to the various advantages of EAMs like low driving voltage and higher bandwidth. Since EAMs are based on electroabsorption, only 


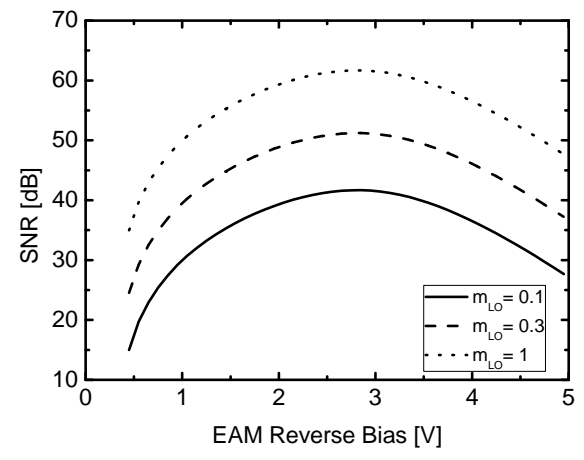

(a)

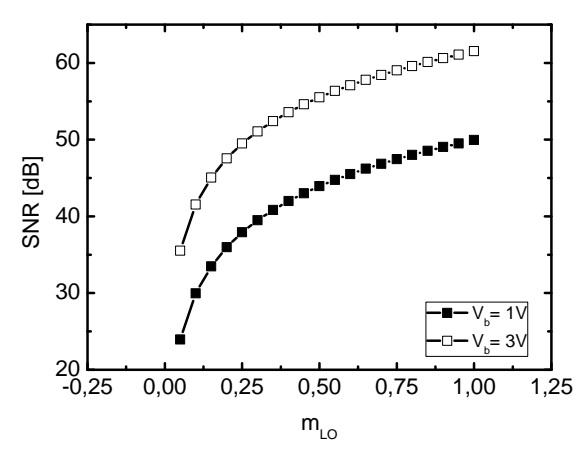

(b)

Figure 2.7: The SNR of the up-converted signals plotted against the EAM reverse bias voltage at different modulation indices(a), and the modulation index (b).

intensity modulation of the optical power can be performed hence only DSB modulation may be generated. Assuming a baseband optical signal from a DML to be up-converted to the RF frequency using a LO signal $V(t)=V_{b}+V_{m} \sin \left(\omega_{L O} t\right)$ in an EAM, the output according to eqn. 2.15 can be expressed as:

$$
E_{\text {out }}=\sqrt{2 L_{E A M}}[P+\eta I(t)]^{\zeta} T^{\chi}(V(t)) \exp j\left(\omega t-\theta_{L}\right)
$$

Where $\zeta=\frac{1-j\left(\alpha_{L}-\alpha_{E}\right)}{2}$ and $\chi=\frac{1+j \alpha_{E}}{2}$. The EAM transfer function $T(V(t))$ can be expressed as Taylor's series around the EAM bias voltage $V_{b}$ according to:

$$
T(V(t))=\frac{1}{n !} \sum_{n=0}^{\infty}\left(\frac{d^{n} T(V)}{d V^{n}}\right)_{V=V_{b}}\left(V(t)-V_{b}\right)^{n}
$$

Expanding $T^{\chi}(V(t))=\exp \left(-\chi K L\left(V-V_{0}\right)^{c}\right)$ using Taylor's series, the output of the EAM can be written as:

$$
\begin{aligned}
E_{\text {out }}= & \sqrt{2 L_{E A M}}[P+\eta I(t)]^{\zeta} T^{\chi}\left(V_{b}\right)\left[1-\chi K L c\left(V_{b}-V_{0}\right)^{c-1} V_{m} \sin \omega_{L O} t\right] \\
& \cdot \exp j\left(\omega t-\theta_{L}\right)
\end{aligned}
$$

The RF and DC photo-current of the above optical signal with a small 
signal modulation $\left(V_{m}<<V_{b}\right)$ is:

$$
\begin{aligned}
i_{R F} & =2 L_{E A M} K L c\left(V_{b}-V_{0}\right)^{c-1} T\left(V_{b}\right)[P+\eta I(t)] V_{m} \sin \left(\omega_{L O} t\right) \\
i_{d c} & =2 L_{E A M} P T\left(V_{b}\right)
\end{aligned}
$$

From eqn. 2.38 it can be noticed that the EAM chirp does not affect the first order signal as observed in [96]. Similar to the MZM based upconversion, SNR of the up-converted signal using EAM is also calculated against the bias voltage and modulation index $\left(m_{L O}=\frac{V_{m}}{V_{b}}\right)$, as shown in fig. 2.7

From the results depicted in fig. 2.7, it may be observed that for optimum performance the EAM bias voltage should be around 2 to 3 volts which depends on the EAM device parameters. Also by increasing the modulation index, the SNR increase, but in practical applications higher modulation index might damage the device or creates significant harmonic distortions, which will be discussed in the next section.

\subsection{Radio Over Fibre Uplinks}

In the previous chapter, up-conversion techniques for broadband wireless links were described, where the wireless signals can be generated centrally in a CS and transmitted to a simple antenna BS in a RoF downlink. In a remote antenna BS, the uplink wireless signals received at the receiver antenna should be transmitted to the CS to complete the link. One way to do this is to demodulate the wireless signals at the BS and transmit the baseband data to the CS. This method would require a RF demodulator at the base station which will complicate the BS configuration. RoF links can be used for uplink transmission by simply modulating the wireless signals on an optical carrier at the BS and transmit to the CS.

Several demonstrations of RoF uplinks have been performed mainly aiming at simplifying the BSs. Some of these links involve reuse of optical carriers of downlink sent from the CS [97], or use of novel devices like Electroabsorption transceivers, which act as PD for downlink and EAM 
for uplink [98]. One of the main limitations of the RoF uplinks is the fibre's chromatic dispersion induced RF power fading, and to mitigate that several techniques were proposed involving conversion of the RF signals to a lower IF frequency signals 99,100 . Another limitation of RoF uplinks is the distortion generated by the modulators, optical fibre, PDs. Since the RF signal is broadband in nature, intermodulation distortion can greatly influence the link performance.

\subsubsection{Photonic Downconversion}

Photonic down-conversion is the process of converting a wireless signal into an optical signal mainly using external modulators and subsequent demodulation as shown in fig. 2.8. Assuming a wireless signal of the form $V_{R F} I(t) \sin \left(\omega_{R F} t+\phi_{R F}(t)\right)$ which contains an amplitude modulation $I(t)$ and a phase modulation $\phi_{R F}(t)$. Modulating this wireless signal on an optical carrier in an infinite extinction ratio MZM, and transmitting over SMF, the optical signal at the CS may be expressed as:

$$
\begin{aligned}
E_{\text {out }}= & \sqrt{2 P T_{f f}}\left[J_{0}\left(m_{R F} \exp j\left(\omega_{0} t+\phi_{0}\right)\right)\right. \\
& \left.+J_{1}\left(m_{R F} \exp j\left(\omega_{0} t+\phi_{0} \pm \omega_{R F}(t) \pm \phi_{R F}(t)\right)\right)\right]
\end{aligned}
$$

The above expression contains the unmodulated optical carrier at $\omega_{0}$ and the two side-bands at $\omega_{0} \pm \omega_{R} F$ which contain the amplitude and phase modulation of the original wireless signal. The chromatic dispersion induced phase shift as seen on the side-bands, which when mixed in a photo-detector will induce the RF power fading. This RF power fading is the limiting factor in RoF uplinks and many techniques have been proposed to mitigate this effect.

\subsubsection{Harmonic Distortion}

The non-linearity of the components in a RoF link distort the signals in most of the applications especially for multi-carrier signal transmission. The non-linear transfer function of these devices create harmonics 


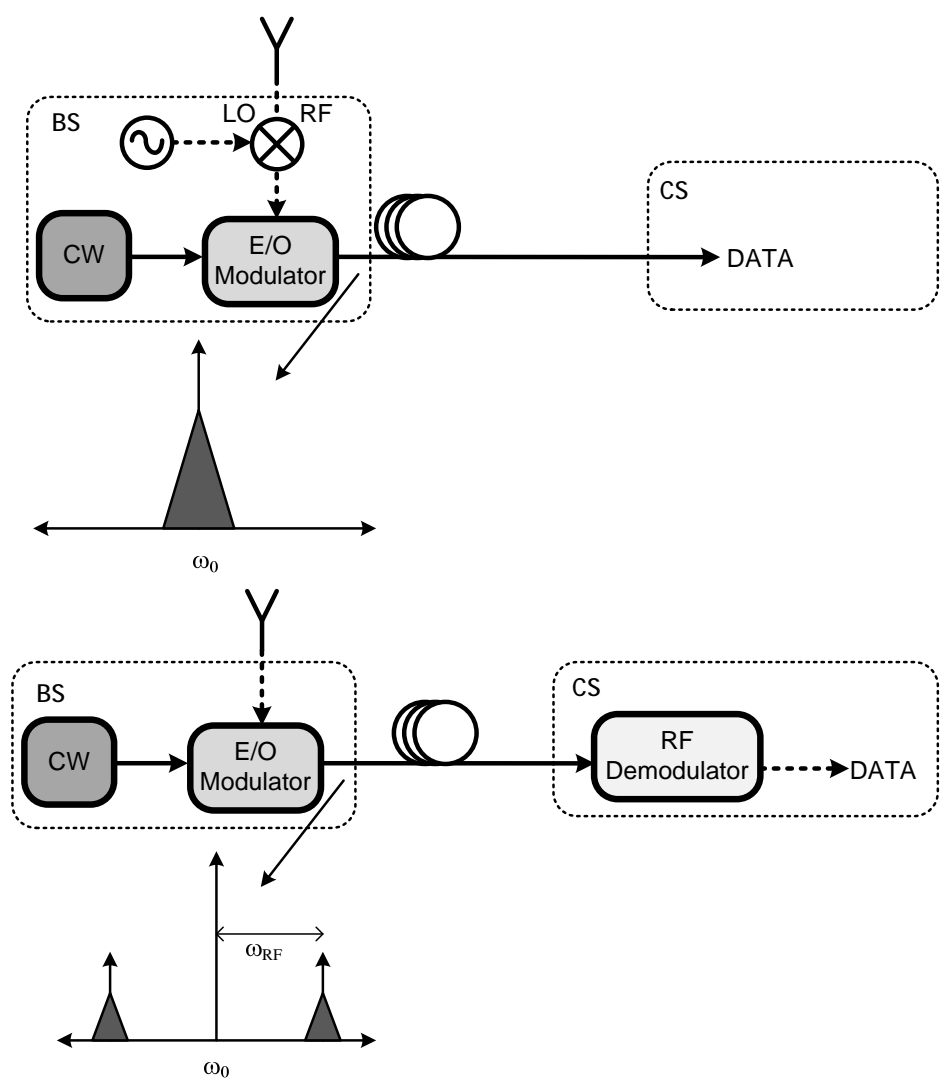

(a)

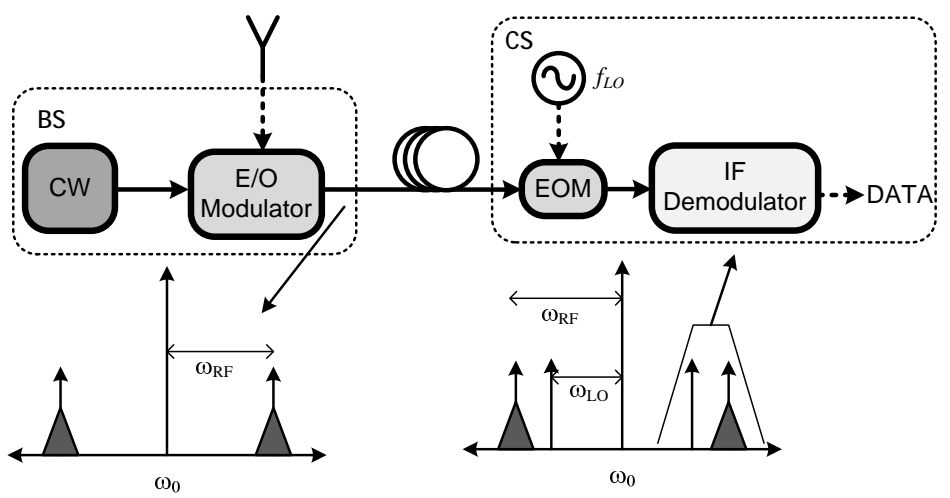

(b)

(c)

Figure 2.8: Schematics of different RoF uplinks. (a) For transmission of baseband data from BS to CS, (b) for transmission directly the RF signals and (c)for transmitting RF signals to the CS and converting to IF signals. 
of the fundamental component and intermodulation products of the fundamental components will hamper the quality of the signal. The power of the harmonics increase with the RF power faster than the fundamental frequency and when the harmonics are above noise level, distortion limits the signal quality. The non-linear characterization of the devices is performed using two-tone analysis where two carriers separated by less than an octave are used and the intermodulation products are measured. The most dominant intermodulation products that determine the system performance are the second order (IMD2) and third order intermodulation (IMD3), and the intermodulation products that occur in-band $\left(2 f_{1}-f_{2}, 2 f_{2}-f_{1}\right)$ are the ones that are to be avoided.

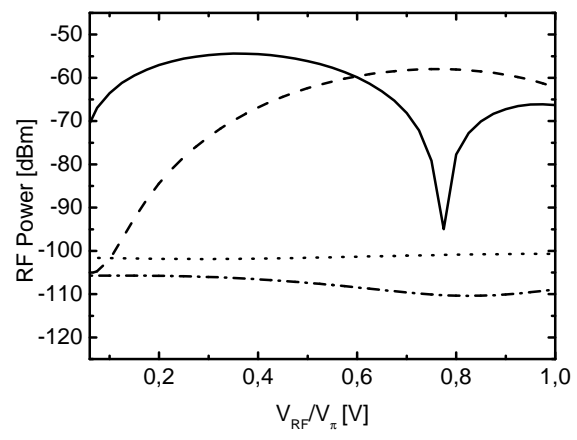

(a)

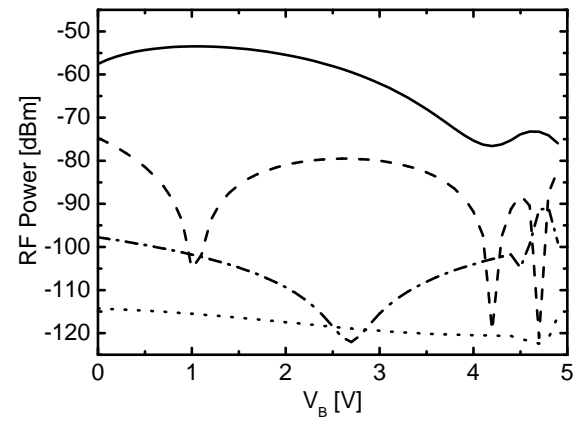

(b)

Figure 2.9: Intermodulation distortion products generated in a MZM at quadrature bias by varying the modulation index (a) and in an EAM by varying the bias voltage (b). Legend: ( - fundamental, - - - second order, - - - third order, $\cdots$ noise.)

The intermodulation distortion is measured using two parameters, the intercept points (IP2, IP3) and the spurious free dynamic range (SFDR). The intercept point is measured by extrapolating the fundamental and the harmonic components, and the intersection of the extrapolated lines is the intercept point. SFDR is the ratio between the fundamental component and the distortion product at the noise level. SFDR of a link determines the dynamic range of a link without inducing intermodulation distortion. The devices used in a RoF such as the $\mathrm{E} / \mathrm{O}$ modulator, the fibre and the PD all add to the distortion of the link. The non linearity of the optical modulator is the main source of harmonic distor- 
tion, which directly depends on the power of the modulating RF signal. The intermodulation distortion from a modulator mainly depends on the modulation index and the bias point, and the results of intermodulation products generated from different from both MZM, and EAM are shown in fig. 2.9.

From fig. 2.9(a) it may be observed that when the MZM is biased at the quadrature point, the dominant harmonic component is the second harmonic which is evident from the eqn. 2.28. In normal QB operation of a MZM, the second order (even order) harmonics are minimized, while at MiTB operation the third order harmonics are minimized. In an EAM, the bias voltage influences the harmonic products, where a bias point can be chosen either to suppress the second or third order component.

\subsubsection{Envelope Detector [Paper A]}

Demodulation of wireless signals typically involve heterodyne techniques, where the received signals are mixed with a phase locked LO signal to demodulate the wireless signals. Recently wireless links with simple ASK modulation format in the $120 \mathrm{GHz}$ band has gained a lot of interest [65], and to demodulate these ASK wireless signals envelope detectors are used, where only the envelope or the amplitude modulation is detected. When electrical envelope detectors are used, a later electrical to optical conversion is required to transmit the ASK data to the CS. Optical envelope detectors on the other hand can simplify the BS configuration by integrating the envelope detection and electrical to optical conversion. Another added advantage of optical envelope detectors is that the detected signals are optical baseband signals which can be fed directly into an optical network without the need for RoF compatibility. Several techniques for envelope detection have been proposed which include the use of baseband photo-detectors, or using gain saturation in a DFB laser in combination with an EAM.

In this work (paper A), a novel optical envelope detection technique is presented as seen in fig. 2.8. Optical envelope detection involves three principal steps: one, the conversion of wireless ASK signals to optics. This is performed using a typical MZM in combination with an optical 
source. The second step is the half wave rectification, since all the RF signals are full wave. The half wave rectification is performed by biasing the MZM in the MiTB as shown in fig. 2.10. The third step is the envelope detection of the half wave rectified optical signal which is simply done by using a typical optical bandpass filter by filtering the side-band, whose output is an optical ASK signal. This whole process is performed only with a MZM and an optical bandpass filter which is a very simple configuration for a BS. To measure the quality of the envelope detected signals, the BER was measured using an optical pre-amplified receiver. The results indicate a very good performance where receiver sensitivity at BER of $10^{-9}$ was measured as $-27 \mathrm{dBm}$.

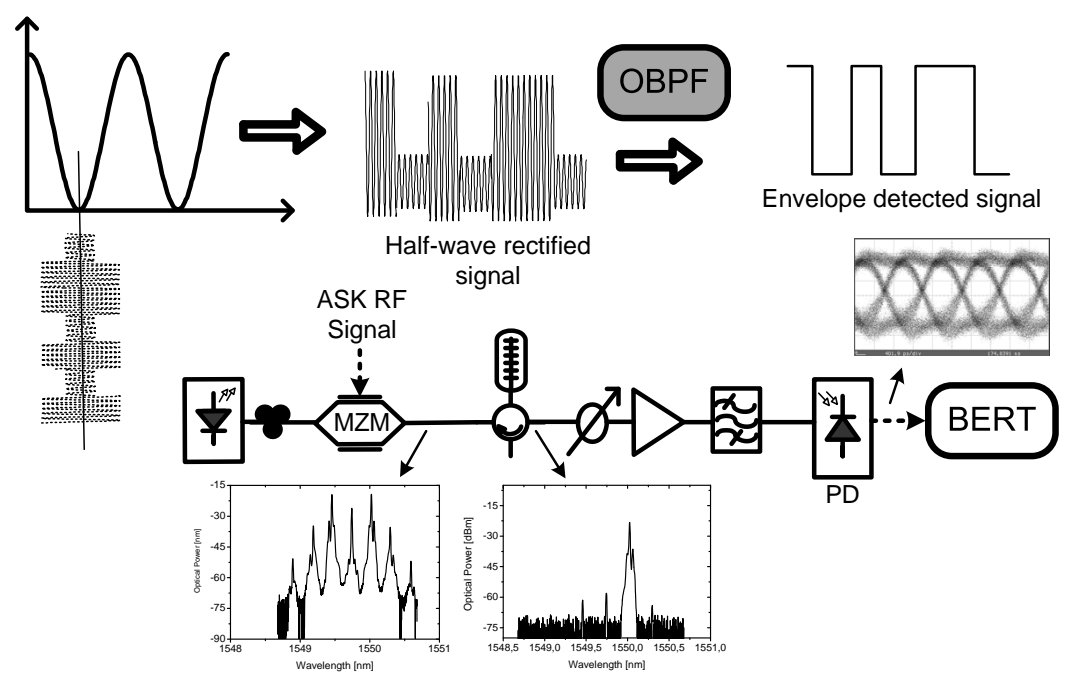

Figure 2.10: Illustration of the optical envelope detection technique, and the experimental set-up for the envelope detector.

\subsection{Conclusions}

In this chapter the basic building blocks of a RoF link were addressed. It is seen that RoF techniques have the potential for generating, transmitting and detecting wireless signals. Direct modulation of laser current offer a good solution for baseband data modulation in RoF links. Some 
of the limitations of the DFB modulation are the low extinction ratio (around 8-10 dB), and the chirp. The extinction ratio of the data modulation directly influences the SNR of the up-converted RF signal.

Optical modulators offer a good choice for up-conversion of baseband signal with high quality, and optical modulators like MZM can be used to generate higher order harmonics where up-conversion to higher frequencies can be achieved using lower frequency LO signals. Up-conversion using a MZM can be performed using two bias conditions, QB and MiTB, where MiTB offers the possibility of frequency doubling but suffers from low SNR (around $5 \mathrm{~dB}$ ) compared to QB. Electroabsorption modulators are another candidate for up-conversion which require low driving voltages compared to a MZM, but have typically higher insertion losses.

The intensity modulation and direct detection nature of RoF up-conversion techniques generates a residual DC photo-current which limits the performance of the signal. The DC photo-current makes the laser RIN as the dominant noise source, and hence optical sources with lower RIN coefficients should be incorporated. Another disadvantage of IM/DD RoF links is the unwanted peak power at the LO frequency, which will saturate the RF amplifiers incorporated in the antenna sites. This unwanted LO peak depends on the data modulation and in near perfect $100 \%$ modulation index the LO peak is $3 \mathrm{~dB}$ higher than data, and a $10 \%$ reduction in the modulation index will increase the LO peak power by around 3 $\mathrm{dB}$. Thus near $100 \%$ data modulation is crucial in reducing the unwanted LO peak power.

The use of external modulators in an RoF uplink was studied in this chapter. It is observed that when a RF signal is modulated on an optical carrier in a DSB modulation, the phase and amplitude of the RF signal is present on the side-bands. This property of the RoF modulation has helped mitigate the fibre's chromatic dispersion induced RF power fading in a typical RoF link. Other than the RF power fading, another limitation of RoF uplinks is the harmonic distortion induced by the various devices, which will mainly affect a broadband wireless signal. As an example of an RoF uplink, a dispersion tolerant optical envelope detector architecture has been proposed in this thesis. The main advantage of this technique is the simplicity where only a MZM and an optical bandpass filter are 
required. The receiver sensitivity measured from the proposed technique is around $-27 \mathrm{dBm}$ which indicates a very sensitive detection technique. 
CHAPTER 3

\section{Photonic Vector Modulation and Demodulation Techniques}

In chapter 2, various RoF techniques for wireless signal generation, transmission and demodulation were presented. To meet the current bandwidth requirements, spectral efficient modulation formats are needed, and for integration of wireless links in optical networks, photonic techniques for generation can provide various advantages. This chapter presents novel photonic vector modulation (PVM) and demodulation (PvDM) concepts for generation, transmission and demodulation of spectral efficient $\mathrm{mmW}$ wireless signals. The PVM concept is based on direct generation of vectorial M-QAM/PSK signals using photonic techniques, whereas PVdM techniques involves the direct conversion of wireless RF signals to baseband demodulated data. First the working principle and different architectures for implementing the vector modulation technique is described in section 3.1. The performance analysis of a photonic vector modulator is numerically evaluated and also discussed in section 3.1. A $10 \mathrm{~Gb} / \mathrm{s}$ wireless link using PVM in the $60 \mathrm{GHz}$ band is present in section 3.2. The use of photonic vector modulation for generation of 16-QAM modulated wireless signals and the various architectures implemented are discussed in section 3.3. The distribution of PVM generated 
wireless signals over a reconfigurable optical network, emulating a RoF link co-existing with other services, is described in section 3.4. The photonic vector demodulation architectures and its performance analysis is described in section 3.5 followed by conclusions in section 3.6.

\subsection{Photonic vector modulation: Working prin- ciple}

In the previous chapter, several up-conversion techniques for up-converting baseband data to the desired RF frequency for generation and optical transmission to a remote BS were addressed. The up-conversion techniques include the most common DSB and DSB-SC techniques which generate RF signals at the electrical LO frequency or at a second harmonic at twice the modulating frequency. The photonic vector modulation is based on the same concept of baseband data modulation and later RF up-conversion. For generating spectral efficient QAM modulation formats, two independent optical baseband signals are up-converted to the RF frequency and using an optical RF phase shifting mechanism, a quadrature phase shift of $90^{\circ}$ is introduced between the two optical components. The photo-detected output of the combined optical signals is an electrical QAM signal at the RF frequency.

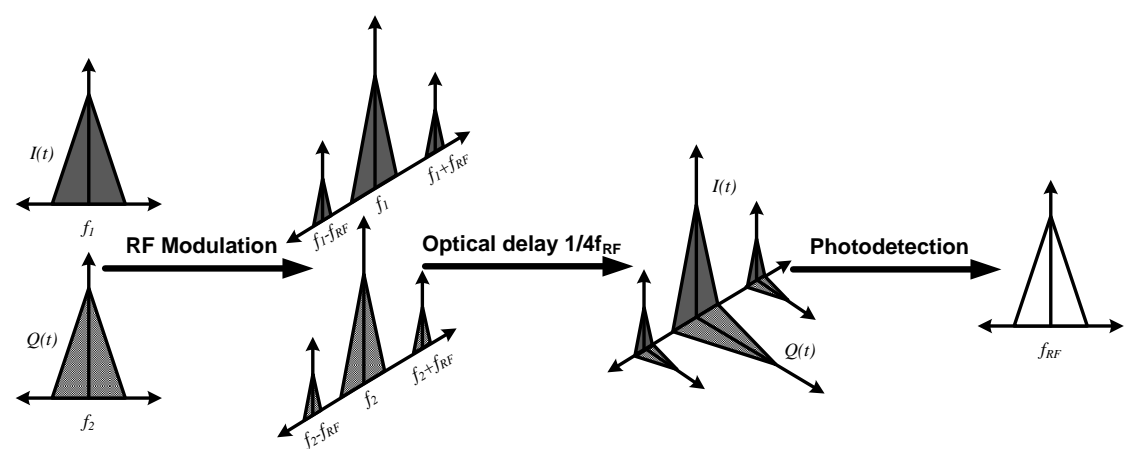

Figure 3.1: Graphical illustration of the concept behind photonic vector modulation. 
Photonic vector modulation is performed in three steps. First two independent baseband data streams $I$ and $Q$ (in-phase and quadrature) modulate two optical carriers ${ }^{1}$ either using directly modulated lasers (DML) or external modulators. DMLs as described in previous chapter, offer a cost-effective solution, whereas external modulators offer better performance, and the choice of modulator depends on the system design. In the second step, the two optical baseband signals are up-converted to the RF frequency using an external modulator either by one of the upconversion techniques (DSB, DSB-SC). The third step in PVM is the quadrature condition for QAM modulation, which is a $90^{\circ}$ phase shift between the $I$ and $Q$ components. This RF phase shift is achieved by introducing an optical delay equivalent to $1 / 4 f_{L O}$ which corresponds to a $90^{\circ}$ electrical phase shift between the two RF carriers at the frequency $f_{L O}$. This optical delay can be introduced either by dispersion or simply using an optical delay line to delay one of the optical carriers. If the optical carriers are at different frequencies, dispersion induces a delay between the spectral components, and tailoring the dispersion according to the optical carriers and the RF frequency, the quadrature condition can be achieved. Optical fibres (SMF) offer the right solution for providing the dispersion, and by either choosing the length or the frequency separation between the optical carriers, the right optical delay can be achieved. The optical carriers with both data and LO modulation with the optical delay included are photo-detected either using single photodiode or balanced photo-receiver, whose output is an electrical signal at the RF frequency with QAM modulation. Figure 3.1 shows the concept of a photonic vector modulator.

\subsubsection{System Architectures [Paper B]}

Photonic vector modulation can be performed in different ways, for e.g either using external data modulators, or DMLs, and either using optical delay lines or dispersion to obtain the RF quadrature phase shift condition. The two principal architectures are shown in fig. 3.2, and small modifications like choice of external modulators or photo-diodes can be made to optimize the system performance. In architecture 1 (fig. 3.2(a))

\footnotetext{
${ }^{1}$ The two optical carriers can be at the same frequency or different depending on the system architecture.
} 
two directly modulated lasers emitting at wavelengths $\lambda_{1}$ and $\lambda_{2}$ are directly modulated by two independent data streams $I$ and $Q$. The two optical carriers are combined and an electrical LO signal at the frequency $f_{L O}$ is used to modulate the optical carriers in an external modulator. Using a SMF, of length $L$, an optical delay of $D L \Delta \lambda$ is introduced due to the SMF's chromatic dispersion. By choosing $L$ or $\Delta \lambda$ the quadrature condition is achieved. The output of the SMF is photo-detected and the resulting signal is a M-QAM signal at the frequency $f_{R F}$.

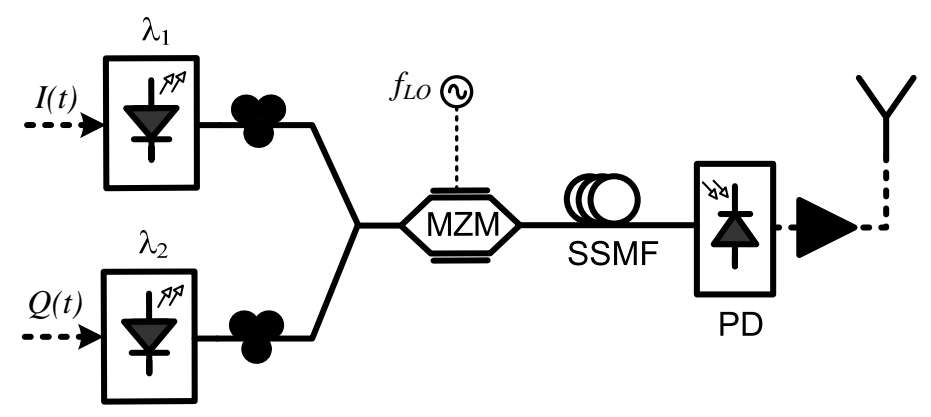

(a)

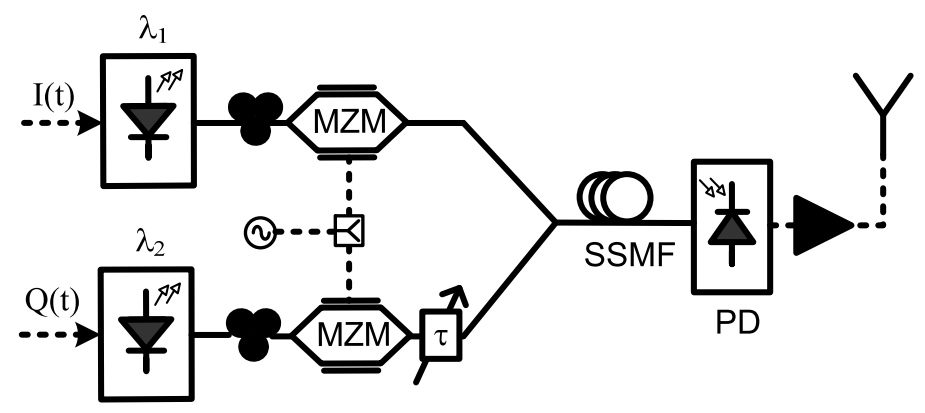

(b)

Figure 3.2: The two architectures of a photonic vector modulator. (a) Using an optical fibre based RF phase shifter, and (b) using an optical delay line as a RF phase shifter.

In the second architecture, as shown in fig. 3.2(b), the two optical baseband data are independently up-converted to the RF frequency using two external modulators. Later by using an optical delay line the quadrature component $(Q)$ is optically delayed by $\Delta T=1 / 4 f_{L O}$. The two optical sig- 
nals are combined and transmitted to the BS using an SMF. The SMF's chromatic dispersion introduces an additional delay between the $I$ and $Q$ components, but by retuning the delay in the optical delay line, the chromatic dispersion induced phase mismatch can be mitigated. The new delay should be $\Delta T \boldsymbol{I}=D L \Delta \lambda-1 / 4 f_{L O}$. The photo-detected output is again a QAM signal.

For the architecture 1, the combined output of the $I$ and $Q$ optical signal (generated by a zero chirp DML) is:

$$
E_{\text {out }}(t)=\sqrt{[P+\eta I(t)]} \exp j\left(\omega_{1} t\right)+\sqrt{[P+\eta Q(t)]} \exp j\left(\omega_{2} t\right)
$$

Using an electrical LO signal of frequency $f_{L O}$ to modulate the optical carriers in a chirp free MZM, the output of the MZM (considering only the fundamental first order components) in a small signal modulation case is:

$$
\begin{aligned}
E_{\text {out }}(t)= & \sqrt{T_{f f}}\left\{\sqrt{[P+\eta I(t)]} \exp j\left(\omega_{1} t\right)\right. \\
& \left.+\sqrt{[P+\eta Q(t)]} \exp j\left(\omega_{2} t\right)\right\}\left[J_{0}\left(m_{L O}\right)\right. \\
& \left.+J_{1}\left(m_{L O}\right) \exp j\left( \pm \omega_{L O} t\right)\right]
\end{aligned}
$$

The RF component of the photo-current is expressed as:

$$
\begin{aligned}
i_{R F}(t)= & 2 \Re L_{t o t} J_{0}\left(m_{L O}\right) J_{1}\left(m_{L O}\right) \cos \left(\frac{\beta_{2} L}{2} \omega_{L O}^{2}\right) \\
& {\left[[P+\eta I(t)] \cos \left(\omega_{L O} t\right)\right.} \\
& \left.+[P+\eta Q(t-\tau)] \cos \left(\omega_{L O} t+\beta_{2} L\left(\omega_{2}-\omega_{1}\right) \omega_{L O}\right)\right]
\end{aligned}
$$

Where $L_{t o t}$ is the total losses of the link, including the MZM insertion losses.

From the eqn. 3.3 it may be noticed that there is a power fading of the RF signal due to the component $\left(\cos \left(\frac{\beta_{2} L}{2} \omega_{L O}^{2}\right)\right)$ which depends on the length of the fibre. Depending on the length of the fibre and the RF carrier frequency, the RF power fading can be mitigated by choosing the fibre length to maximize the RF power, and then tune the frequency separation between the optical carriers $\left(\omega_{2}-\omega_{1}\right)$ for the quadrature condition. For 
maximizing the RF power, the following condition must be satisfied:

$$
\begin{aligned}
\frac{\beta_{2} L}{2} \omega_{L O}^{2} & =n \pi, \forall n=1,2 \cdots \\
L & =\frac{n \pi}{\beta_{2} \omega_{L O}^{2}}
\end{aligned}
$$

For a quadrature condition in a QAM modulation, the following condition must be satisfied in eqn. 3.3 .

$$
\beta_{2} L \Delta \omega \omega_{L O}=\frac{(2 m-1) \pi}{2}, \forall m=1,2 \cdots
$$

From eqn. 3.4 and eqn. 3.5 , the relation between the the frequency separation and RF carrier frequency can be expressed as:

$$
\left(\omega_{2}-\omega_{1}\right)=\frac{2 m-1}{2} \omega_{L O}
$$

To avoid the frequency interleaving of the side-bands on the optical carriers, the minimum separation between the optical signal should be $\left(\omega_{2}-\omega_{1}\right)>\omega_{L O}$. The output of the PD, when the quadrature condition is satisfied is:

$$
\begin{aligned}
i_{R F}(t)= & 2 \Re L_{t o t} J_{0}\left(m_{L O}\right) J_{1}\left(m_{L O}\right) \cos \left(\frac{\beta_{2} L}{2} \omega_{L O}^{2}\right) \\
& {\left[[P+\eta I(t)] \cos \left(\omega_{L O} t\right)+\left[P+\eta Q\left(t-t^{\prime}\right)\right] \sin \left(\omega_{L O} t\right)\right] }
\end{aligned}
$$

Similarly, when an optical delay is used as shown in fig. 3.2(b), the photocurrent at the output is expressed as:

$$
\begin{aligned}
i_{R F}(t)= & 2 \Re L_{t o t} J_{0}\left(m_{L O}\right) J_{1}\left(m_{L O}\right) \cos \left(\frac{\beta_{2} L}{2} \omega_{L O}^{2}\right)\left[[P+\eta I(t)] \cos \left(\omega_{L O} t\right)\right. \\
& \left.+\left[P+\eta Q\left(t-t^{\prime}\right)\right] \cos \left(\omega_{L O}(t-\tau)+\beta_{2} L\left(\omega_{2}-\omega_{1}\right) \omega_{L O}\right)\right]
\end{aligned}
$$

For obtaining $90^{\circ}$ phase shift in the case when an optical delay line is used, the following condition must be satisfied:

$$
\beta_{2} L\left(\omega_{2}-\omega_{1}\right) \omega_{L O}-\omega_{L O} \tau=\frac{(2 m-1) \pi}{2}
$$

The use of the optical delay line relaxes the requirements on the optical carriers' frequency separation which can be mitigated by the delay $\tau$. 
From the above equation (eqn. 3.9 ), the expression in eqn. 3.8 becomes similar to eqn. 3.7 .

There is a delay offset $t^{\prime}$ between the $I$ and $Q$ data streams which is relatively small compared to the bit period of the data stream, as long as the carrier frequency is high. This delay can be corrected by introducing a delay in the electrical data stream prior to modulation. Both the PVM architectures can be implemented using the DSB-SC up-conversion technique to generate the RF wireless signals without changing the phase shifter configuration. The advantage of using DSB-SC technique is the absence of optical carrier, which in a WDM scenario will reduce the total power input into the fibre. Experimental demonstration of wireless signal generation and subsequent demodulation using both the PVM architectures were performed and reported in Papers B and C. Figure 3.3 shows the RF spectra and the demodulated eye diagrams of a QPSK wireless signal in the $40 \mathrm{GHz}$ band, showing the potential of the PVM technique.
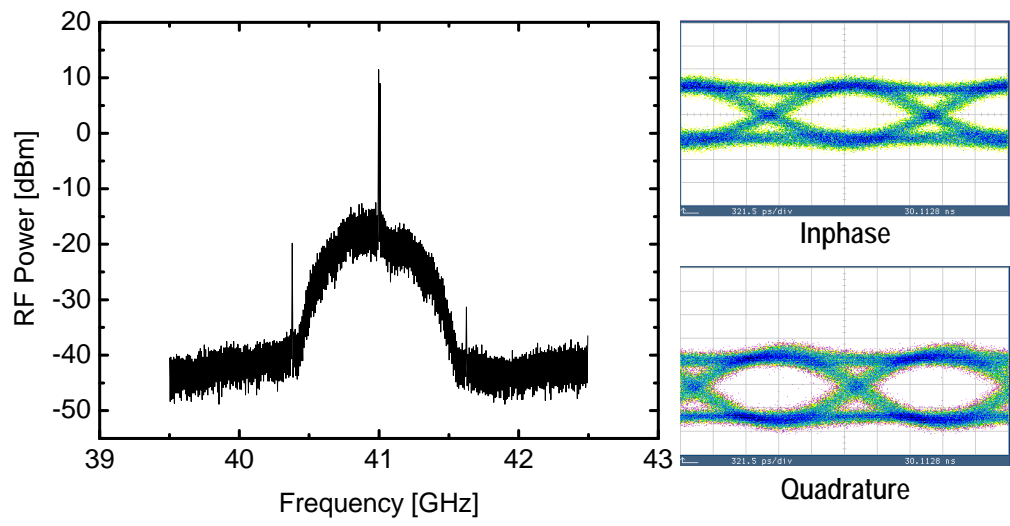

Figure 3.3: Experimental results (from architecture 1) of a $1.25 \mathrm{~Gb} / \mathrm{s}$ QPSK wireless signal in the $40 \mathrm{GHz}$ band. On the left the RF spectrum and on the right the in-phase and quadrature demodulated eye diagrams [Paper B]. 


\subsubsection{LO Peak Suppression [Paper D]}

From fig. 3.3 (RF spectrum) and eqn. 3.7 it may be observed that the generated QAM RF wireless signals have a very high unmodulated peak at the LO frequency, which will saturate the power amplifiers in the wireless transmission system limiting the output power and the total wireless transmission distance. In the chapter 2 it was observed that this unmodulated LO peak is due to the data modulation index and depends on the modulating current and the laser bias point. The component in the RF current (eqn. 3.7) that contributed to this unmodulated peak is $P\left(\cos \left(\omega_{L O} t\right)+\sin \left(\omega_{L O} t\right)\right)$. To suppress this component, another laser with only LO modulation may be added to the PVM system which will counteract the high LO peak. Figure 3.4 shows the schematic of the modified PVM architecture. The photo-current at the output of the modified architecture is:

$$
\begin{aligned}
i_{R F}(t)= & 2 \Re L_{t o t} J_{0}\left(m_{L O}\right) J_{1}\left(m_{L O}\right) \cos \left(\frac{\beta_{2} L}{2} \omega_{L O}^{2}\right)\left[[P+\eta I(t)] \cos \left(\omega_{L O} t\right)\right. \\
& +[P+\eta Q(t-\tau)] \cos \left(\omega_{L O} t+\beta_{2} L\left(\omega_{2}-\omega_{1}\right) \omega_{L O}\right) \\
& \left.+P_{3} \cos \left(\omega_{L O} t+\beta_{2} L\left(\omega_{3}-\omega_{1}\right) \omega_{L O}\right)\right]
\end{aligned}
$$

Where $P_{3}$ is the power, and $\omega_{3}$ the frequency of the third laser. Thus, from the eqn. 3.7 and eqn. 3.10 the condition for LO peak suppression is:

$$
\begin{aligned}
P_{3} & =\sqrt{2} P \\
\left(\omega_{3}-\omega_{1}\right) & =2.5\left(\omega_{2}-\omega_{1}\right)
\end{aligned}
$$

Following this condition, the LO peak is completely suppressed, where the spectrum of the pure QAM signal can be seen in fig. 3.4. The addition of the new laser increases the optical power, and the total noise generated, which will be evaluated in the following section.

\subsubsection{Performance Evaluation [Paper C]}

In the previous sections architectures for QAM wireless signal generation using PVM techniques are presented, and also a modified architecture to mitigate the LO peak is described. The performance of the PVM architectures depend on several system parameters, and must be identified 


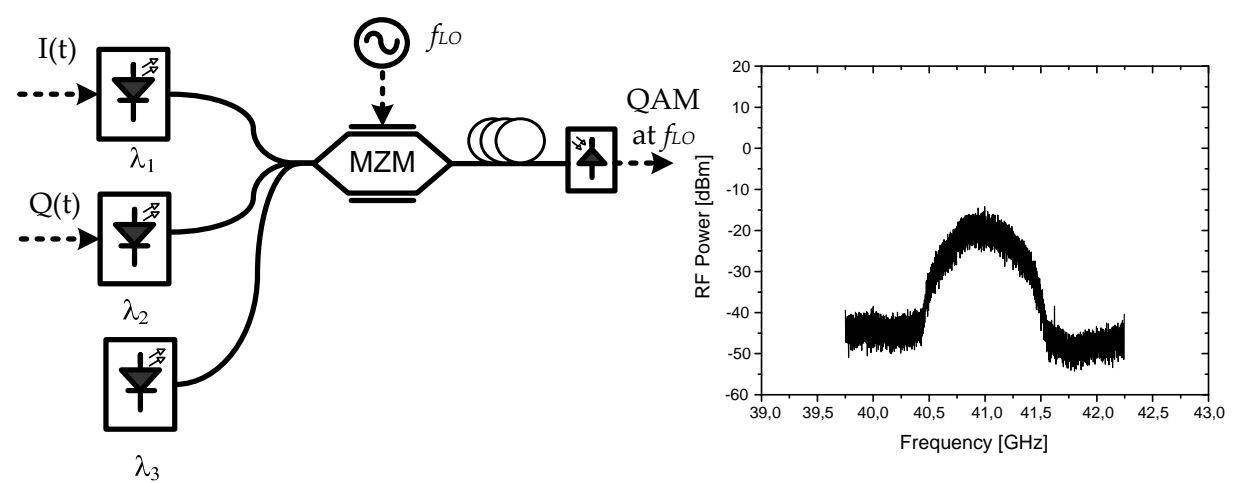

Figure 3.4: The modified architecture for suppression of high unmodulated LO peak (left), and the RF spectrum of the pure QAM signal (right).

and the performance evaluated to form some basic design guidelines. In paper $\mathrm{C}$ the performance of both the PVM and LO suppressed PVM architectures are evaluated both numerically and experimentally and this section summarizes the results obtained.

The main limitation of any communication system is the noise, and it is crucial to identify the noise sources and the parameters that influence it. In chapter 2, the influence of noise on an up-converting RoF link was analysed and in principle is the same for a PVM architecture since the up-conversion technique involved is the same. The main noise contribution in a RoF link (IM/DD) are the laser RIN, the shot noise and the thermal noise. The total noise power directly depends on the DC photocurrent, which depends on the optical power and the LO modulation index. The signal to noise ratio (SNR) depends on the data modulation index and the LO modulation index. In analysing the performance of the PVM architectures 100\% data modulation index is assumed. The main parameters that contribute to the overall SNR of the signal are the total optical losses, the LO modulation index, and the data power. Figure 3.5 summarizes the results of numerical calculation of the SNR influenced by the aforementioned parameters.

From the fig. 3.5 the main conclusion one can draw is that the laser RIN determines to a great extent the total SNR of the signal. This is due to the IM/DD nature of the up-conversion techniques incorporated. To reduce 
the influence of the laser's intensity noise, lasers with RIN parameters of around -140 to $-155 \mathrm{~dB} / \mathrm{Hz}$ should be used. The SNR is very low in a RIN dominant situation, and from fig. 3.5.a) it can be seen that a difference in SNR of around $20 \mathrm{~dB}$ is observed when the RIN parameter is changed

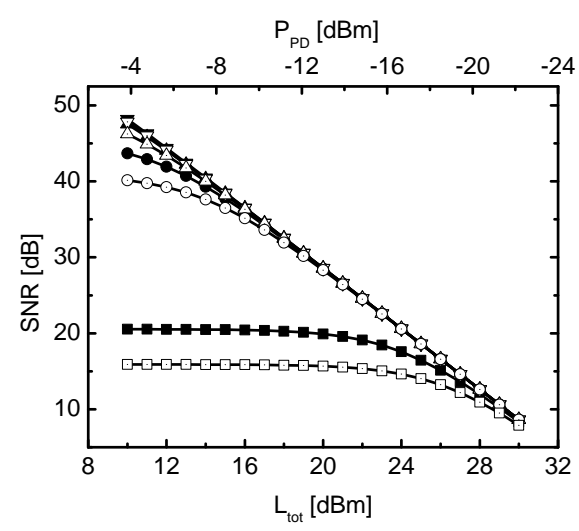

(a)

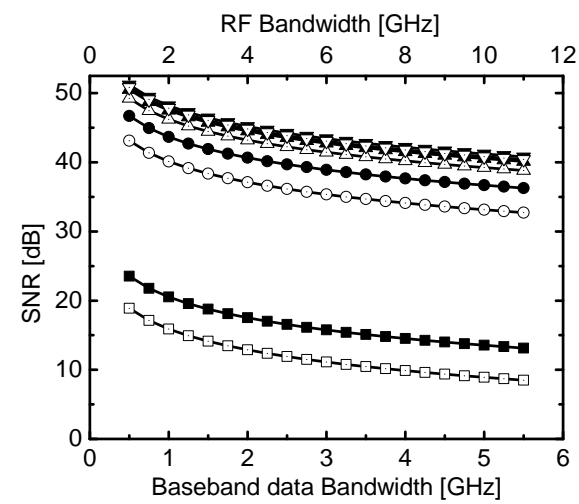

(c)

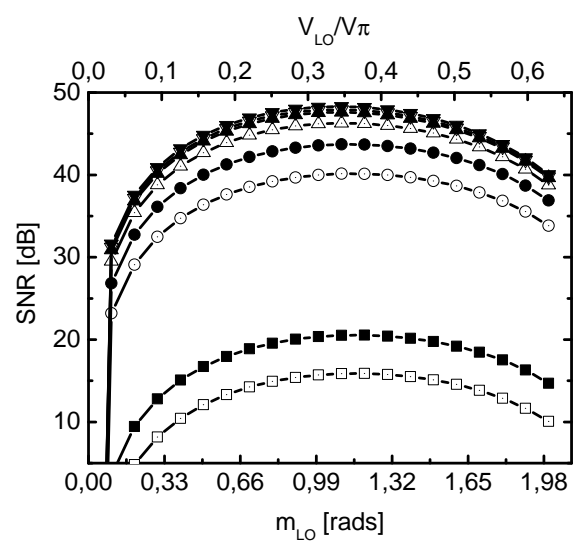

(b)

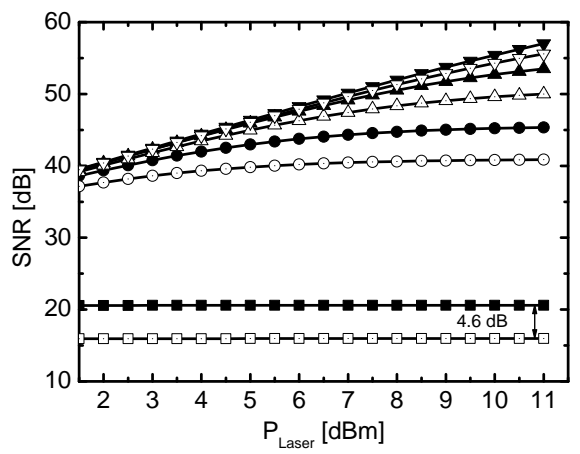

(d)

Figure 3.5: Results of the numerical performance analysis of a QAM signal generated by PVM architectures. Plots contain the variation of SNR on the total optical losses (a), the LO modulation index (b), the baseband data bandwidth (c), and the data laser power (d) for different RIN values.(legend: RIN $\square-110$ $\mathrm{dB} / \mathrm{Hz}, \odot-135 \mathrm{~dB} / \mathrm{Hz}, \triangle-145 \mathrm{~dB} / \mathrm{Hz}, \nabla-155 \mathrm{~dB} / \mathrm{Hz}$. The filled symbols represent the conventional QAM signal, whereas the empty symbols represent the LO suppressed QAM signal.) 
from -110 to $-145 \mathrm{~dB} / \mathrm{Hz}$. In a system dominant by other noise sources like thermal or shot, the SNR directly varies with the total optical power as observed in fig. 3.5(a). Similar to the conclusions drawn in chapter 2, the optimum modulation index for RF up-conversion is around $\pi$.

Another conclusion that may be drawn is that the LO suppressed architecture where the third unmodulated laser is incorporated reduces the SNR especially in a RIN dominant condition. This is evident by the fact that the addition of third laser increases the RIN contribution to the noise. On the other hand, in a shot noise limited system, the addition of the third laser has very less SNR degradation. The effect of data modulation index (laser power) is depicted in fig. 3.5(d), where the increased extinction ratio increases the SNR but only when at lower RIN parameters. For example in the case where the RIN parameter is -110 $\mathrm{dB} / \mathrm{Hz}$, no increment in SNR is observed when increasing the data modulation extinction ratio. In conclusion, due to the IM/DD nature of the up-conversion lasers with lower RIN parameters are crucial for achieving high performance.

The performance of the PVM architectures is also experimentally analysed, since some parameters like laser harmonic distortion were not considered in the numerical analysis. Both the PVM architectures with two up-conversion techniques have been investigated: using QB for DSB based up-conversion and MiTB for second harmonic generation using DSB-SC modulation. To analyse the quality of the PVM generated QPSK wireless signals, Q-factors of the demodulated $I$ and $Q$ data were varied with the laser's data modulation current while maintaining the data modulation near $100 \%$. Also the LO power to the MZM was varied and the fig. 3.6 plots both the results. From the results obtained it may be observed that the increase in the laser modulation current (thus laser power) the signal quality improves, but at very high modulation currents, the laser starts distorting which is evident by the Q-factor penalty after certain laser power. Also it may be observed that the QB has a better performance compared to the MiTB which is in agreement with the conclusions drawn in chapter 2 . 


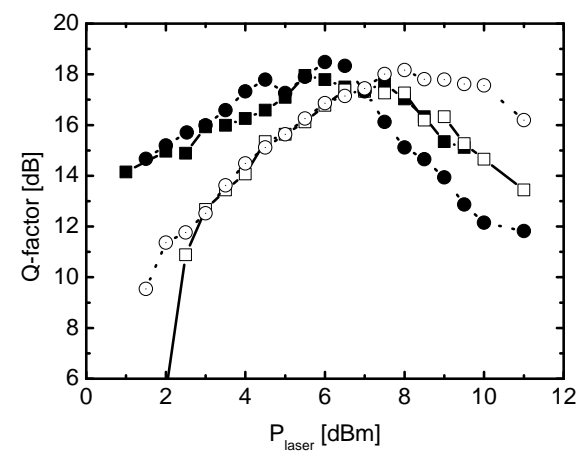

(a)

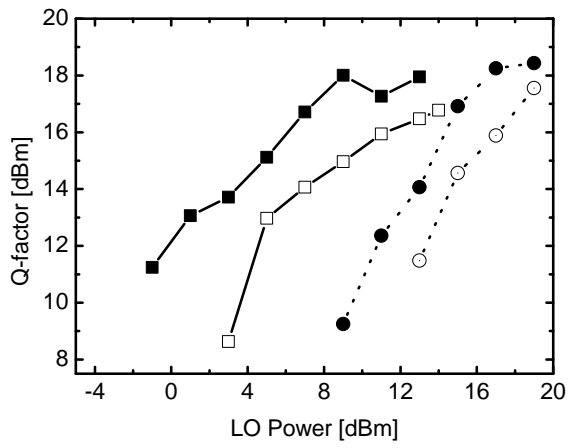

(b)

Figure 3.6: Measured Q factors of a the PVM architectures with both QB and MiTB bias conditions against the laser data power (a) and the LO power (b). (Legend: $\square \mathrm{QB}, \square \mathrm{MiTB}, \bullet \mathrm{LO}$ suppressed at QB, $\odot$ LO suppressed at MiTB.)

\section{$3.260 \mathrm{GHz}$ Wireless Links}

In the previous sections the performance of a PVM based QAM wireless signal generator was evaluated and it is seen that high SNR QAM signals can be generated using the PVM technique. In this section an experimental demonstration of a $10 \mathrm{~Gb} / \mathrm{s}$ wireless link with QPSK modulation in the $60 \mathrm{GHz}$ frequency band using PVM is described. The frequency band of $60 \mathrm{GHz}$ is of special interest in wireless communications because of its free licensing. Another factor that is interesting in the $60 \mathrm{GHz}$ band is its huge undivided bandwidth of $7 \mathrm{GHz}$. The main applications for $60 \mathrm{GHz}$ that are currently under consideration are indoor communications, but short to medium range high capacity wireless links is another emerging application. One of the main motivation of outdoor $60 \mathrm{GHz}$ wireless links is for extending the reach of fibre in a cost-effective manner. RoF links for $60 \mathrm{GHz}$ applications are being investigated for various mobile/broadband services including high capacity wireless links which is well supported by recent advances in photonic devices like EAMs and PDs in the $60 \mathrm{GHz}$ band. One of the major drawbacks of $60 \mathrm{GHz}$ is its high oxygen $\left(\mathrm{O}_{2}\right)$ attenuation, which greatly limit the transmission distance in outdoor wireless links. To quantify the maximum possible wireless distance, the link availability requirements, and link attenuation 
for a specific geographical location must be calculated.

\subsubsection{Link Availability and Link Budget Calculations}

Most of the $\mathrm{mmW}$ wireless links suffer from rain attenuation, and before designing a link, the amount of rainfall in a certain geographic location must be estimated. The link availability is the probability that the link will provide undisrupted communication. For most of the applications $99.999 \%$ also referred as "five nines" is required. When a geographical location receives high rainfall poor link availability is obtained. Link attenuation is the total loss in the wireless path due to all the various loss mechanism. The free space loss at $60 \mathrm{GHz}$ can be expressed as:

$$
\alpha_{F S}(d B)=20 \log (d)+20 \log (f)-147.56
$$

Where $d$ is the wireless link distance measured in meters, and $f$ the carrier frequency measured in Hertz.

Assuming a link distance of $1 \mathrm{~km}$, the free space path loss $\alpha_{F S}$ is calculated to be around $128 \mathrm{~dB}$. The oxygen attenuation at $60 \mathrm{GHz}$ is around $15 \mathrm{~dB} / \mathrm{km}$. In geographical locations with moderate rainfall (100 $\mathrm{mm} / \mathrm{hr}$ ), for $99.999 \%$ link availability [101], the rain induced attenuation is around $30 \mathrm{~dB} / \mathrm{km}$. The total path loss at $60 \mathrm{GHz}$ for $1 \mathrm{~km}$ wireless link is around $170 \mathrm{~dB}$. At $60 \mathrm{GHz}$ due to its narrow beam width, high gain antennas with gain upto $57 \mathrm{dBi}$ can be designed which can enable wireless links up to more than $1 \mathrm{~km}$ of distance.

\subsubsection{Photonic Vector Modulated $10 \mathrm{~Gb} / \mathrm{s} 60 \mathrm{GHz}$ QPSK Wireless Link}

For integrated wireless/wired connectivity, development of wireless links with $10 \mathrm{~Gb} / \mathrm{s}$ capacity is crucial. Currently, electrical technology is not mature enough for providing $10 \mathrm{~Gb} / \mathrm{s}$ wireless links, especially in the mmW frequency bands. $60 \mathrm{GHz}$ band with its $7 \mathrm{GHz}$ of bandwidth is a right candidate for providing such high capacity wireless links, if spectral 


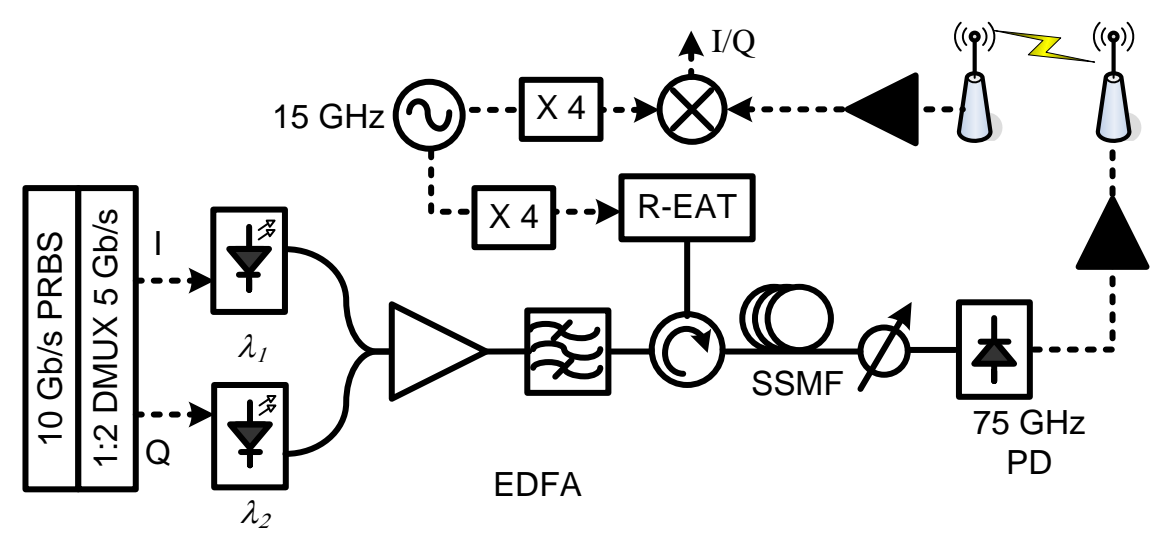

Figure 3.7: Schematic of a $10 \mathrm{~Gb} / \mathrm{s}$ photonic vector modulated QPSK wireless link in the $60 \mathrm{GHz}$ frequency band.

efficient QAM modulation formats are used. Photonics has the advantage of not only providing the required bandwidth, but also allow easy integration of such wireless signals in an optical network. Photonic vector modulated wireless links as seen in the previous section have the capacity to generate high bit-rate QAM wireless signals, and in the European FP6 project IST-IPHOBAC ${ }^{2}$ demonstration of a $10 \mathrm{~Gb} / \mathrm{s}$ wireless link was one of the main goals.

Figure 3.7 shows the experimental set-up for of a wireless link consisting of a PVM architecture for generation of a $10 \mathrm{~Gb} / \mathrm{s}$ QPSK wireless link in the $60 \mathrm{GHz}$ band. Two DFB directly modulated lasers separated $500 \mathrm{GHz}$ apart are directly modulated by two independent non-return-to-zero (NRZ) $5 \mathrm{~Gb} / \mathrm{s}$ (electrical bandwidth of $3.75 \mathrm{GHz}$ ) data streams. Using a $60 \mathrm{GHz}$ bandwidth reflective EAM, a $60 \mathrm{GHz}$ LO signal was modulated on the two optical carriers. Using a SMF of length $62 \mathrm{~m}$, the quadrature phase shift condition was obtained. For generating the wireless signals, a $75 \mathrm{GHz} \mathrm{PD}$ was used after the $62 \mathrm{~m}$ of SMF. The output of the PD is $60 \mathrm{GHz} 10 \mathrm{~Gb} / \mathrm{s}$ wireless signal with a total occupied bandwidth of 7.5 GHz. For wireless transmission, the output of the PD was amplified using a $10 \mathrm{GHz}$ bandwidth low noise amplifier with a gain of $17 \mathrm{~dB}$, and radiated using a $20 \mathrm{dBi}$ gain horn antennas. After $2 \mathrm{~m}$ of wireless transmission (94 dB of free space loss), the wireless $10 \mathrm{~Gb} / \mathrm{s}$ signal was

\footnotetext{
${ }^{2}$ www.ist-iphobac.org
} 
received using another $20 \mathrm{dBi}$ gain horn antenna and amplified using a high power amplifier with a gain of $26 \mathrm{~dB}$. The amplified signals at the receiver were demodulated using an electrical $60 \mathrm{GHz}$ mixer multiplied with a copy of the LO signal.

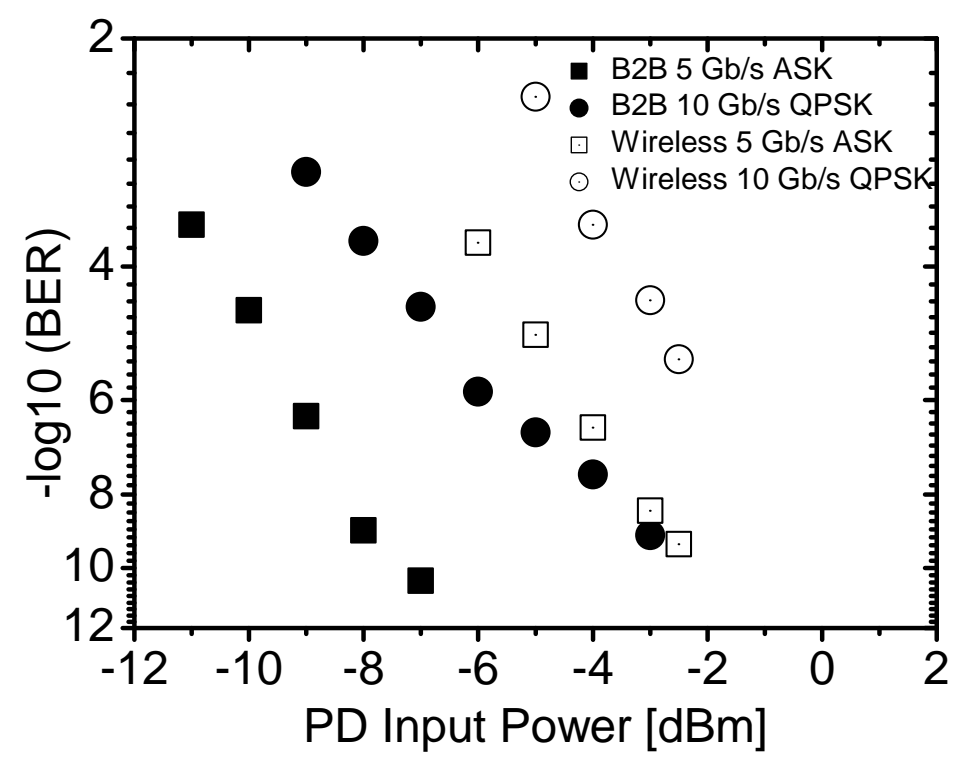

Figure 3.8: Bit error ratio plots of a $10 \mathrm{~Gb} / \mathrm{s} 60 \mathrm{GHz}$ wireless signals.

To analyse the quality of the signal, first the bit error ratio (BER) of the demodulated signals was measured in a back-to-back scenario, without any wireless transmission. For the back-to-back case, the transmitter and receiver amplifier were directly cascaded without the antennas. The BER was measured by varying the optical power input to the PD. To estimate the penalty induced by the PVM with the quadrature modulation, the $10 \mathrm{~Gb} / \mathrm{s}$ QPSK signals were compared to a $5 \mathrm{~Gb} / \mathrm{s}$ ASK signals. The reference ASK signals were generated by simply switching off one of the lasers. Similarly, after the wireless transmission the BER of both 10 $\mathrm{Gb} / \mathrm{s}$ QPSK and $5 \mathrm{~Gb} / \mathrm{s}$ ASK signals were measured as shown in fig. 3.8 . A $3 \mathrm{~dB}$ power penalty (for BER of $10^{-6}$ ) between the $10 \mathrm{~Gb} / \mathrm{s}$ QPSK and $5 \mathrm{~Gb} / \mathrm{s}$ ASK signals is recorded. This power penalty is because of two reasons, one the total RF power of a QPSK signal is lower compared to an ASK signal for the same optical input power to the PD, and second 
the QAM demodulation requires an accurate phase locking mechanism, and in the demodulation process, the phase locking was achieved manually using a phase shifter. After the wireless transmission with additional $94 \mathrm{~dB}$ of loss in the link, a $5 \mathrm{~dB}$ power penalty was observed compared to the back-to-back case. By adding additional amplifier stages and using high gain antennas, the transmission distance of the wireless signals can be drastically increased.

To transmit $10 \mathrm{~Gb} / \mathrm{s}$ in the $7 \mathrm{GHz}$ available bandwidth at $60 \mathrm{GHz}$, some pulse carving techniques can be used to reduce the electrical bandwidth of the $5 \mathrm{~Gb} / \mathrm{s}$ data signals. Also pre-equalization techniques can be implemented in combination with pulse shaping to reduce the total bandwidth of the signal. In real scenarios the uplink requires lower bandwidth compared to downlink, and for implementation of a bi-directional wireless link, another frequency bands like $40 \mathrm{GHz}$ can be used for the uplink. Another solution for providing bi-directional links in the $60 \mathrm{GHz}$ band is the use of higher order M-ary QAM modulation formats like 16-QAM for the downlink.

\subsection{M-ary Quadrature Amplitude Modulation}

To increase the bit rate of communication links without increasing the occupied bandwidth, higher order spectral efficient modulation formats can be implemented. In the previous section, PVM architectures for generation of 4-QAM/QPSK were described, which has a spectral efficiency of around $1.33 \mathrm{~b} / \mathrm{s} / \mathrm{Hz}^{3}$. For generating QPSK modulated wireless signals using PVM architectures, the in-phase and quadrature optical signals were modulated by binary data for generating 2-level I and Q optical signals and for M-QAM signals, multi-level optical I and Q should be generated. As shown in fig. 3.9 there are two ways of generating the multilevel baseband optical signals, either by creating a multilevel electrical signal and then modulating on an optical carrier, or directly generating an optical multilevel signal by using $n$ lasers with binary modulation. The important parameter that determines the quality of a multilevel

\footnotetext{
${ }^{3}$ The spectral efficiency is calculated for NRZ pulses which require electrical bandwidth of around $75 \%$ of the bit-rate.
} 


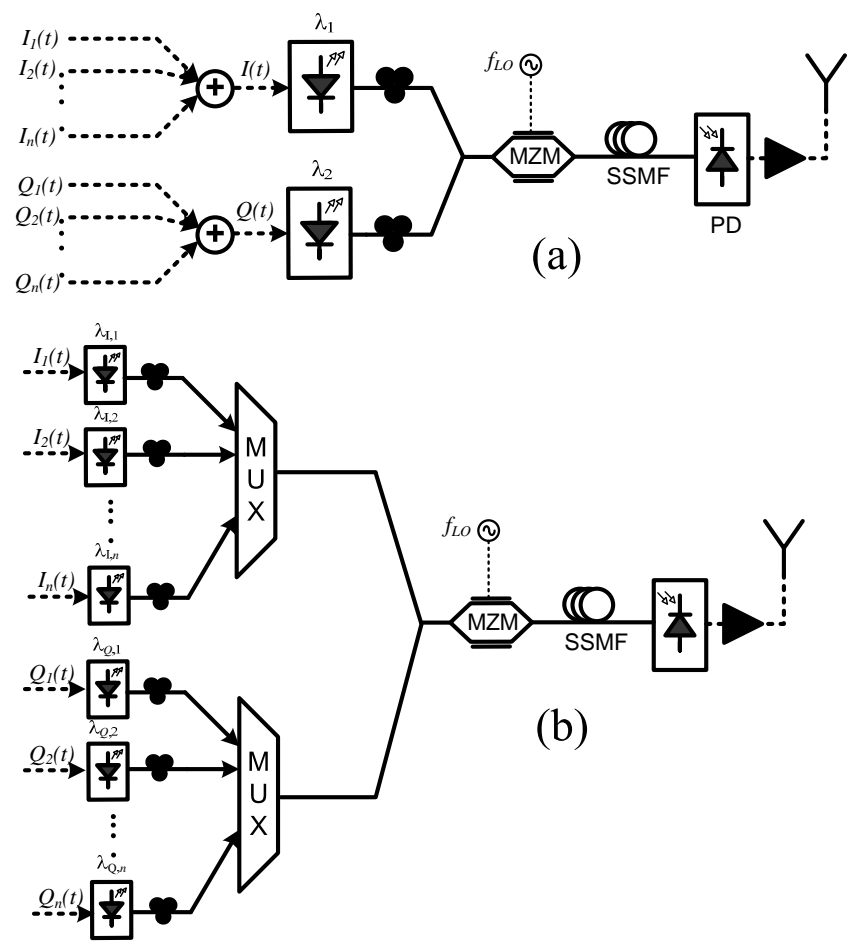

Figure 3.9: Schematics for generation of M-ary QAM wireless signals using photonic vector modulator.

signal is the data modulation extinction ratio. When an electrical multilevel signal is used to modulate a DML, the extinction ratio reduces as the number of levels increases. While when using multiple optical signals, the extinction ratio will be higher. External modulators instead of DMLs can help to mitigate the effect of extinction ratio since external modulators typically have an extinction ratio of around $20 \mathrm{~dB}$ compared to the 8-10 dB extinction ratio of DMLs.

\subsubsection{6-QAM Wireless Signal Generation [Paper E, F, $\mathrm{G}]$}

In the previous section, a wireless link of $10 \mathrm{~Gb} / \mathrm{s}$ within a bandwidth of 7.5 GHz using QPSK modulation was described. To increase the bit-rate 


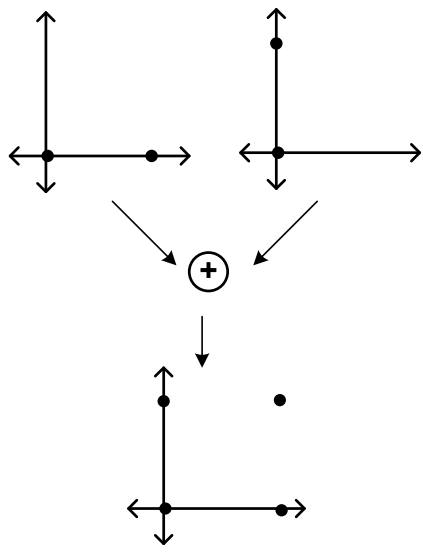

QPSK
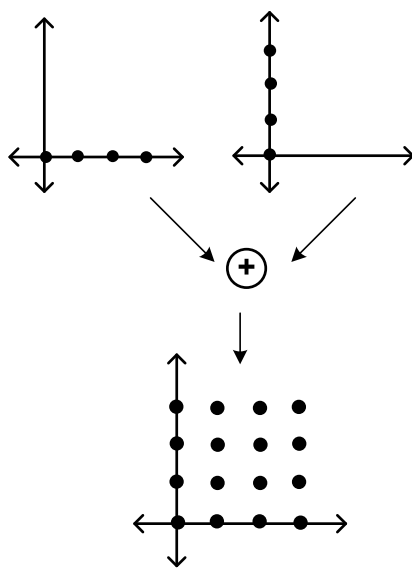

16-QAM

Figure 3.10: Illustration of QPSK and 16-QAM signalling for photonic vector modulated wireless signal generation.

or to reduce the electrical bandwidth higher level $(M>4)$ modulation formats should be used. 16 level modulation formats offer twice the spectral efficiency compared to the 4 level QPSK modulation format. Using PVM architectures 16 level QAM modulation formats can be easily implemented. In the PVM architecture for QPSK signal generation, the $I$ and $Q$ data signals were binary in nature (2 levels) and by changing the $I$ and $Q$ signalling from binary to 4 level amplitude, 16-QAM modulation can be generated, as shown in fig. 3.10. 16-QAM modulation format has twice the spectral efficiency with only $3 \mathrm{~dB}$ higher SNR requirements compared to QPSK modulation format. In papers $\mathrm{E}, \mathrm{F}$, and $\mathrm{G}$, different architectures for generation of $16-\mathrm{QAM}$ modulated $\mathrm{mmW}$ wireless signals are presented. In papers $\mathrm{E}$ the 16-QAM signal generation is based on the techniques showed in fig. $3.9 \mathrm{k}$, where a multi-level electrical signal is modulated on the $I$ and $Q$ lasers, where both LO suppressed and unsuppressed architectures were implemented. Papers F and G present 16-QAM signal generation using independent optical binary signals according to the architecture shown in fig. $3.9 \mathrm{~b}$.

In paper $\mathrm{G}$, a $10 \mathrm{~Gb} / \mathrm{s}$ 16-QAM wireless signal generation using PVM is presented. Figure 3.11 shows the overview of the experimental set-up and the results obtained (Paper G). In this system, first the LO is modulated 

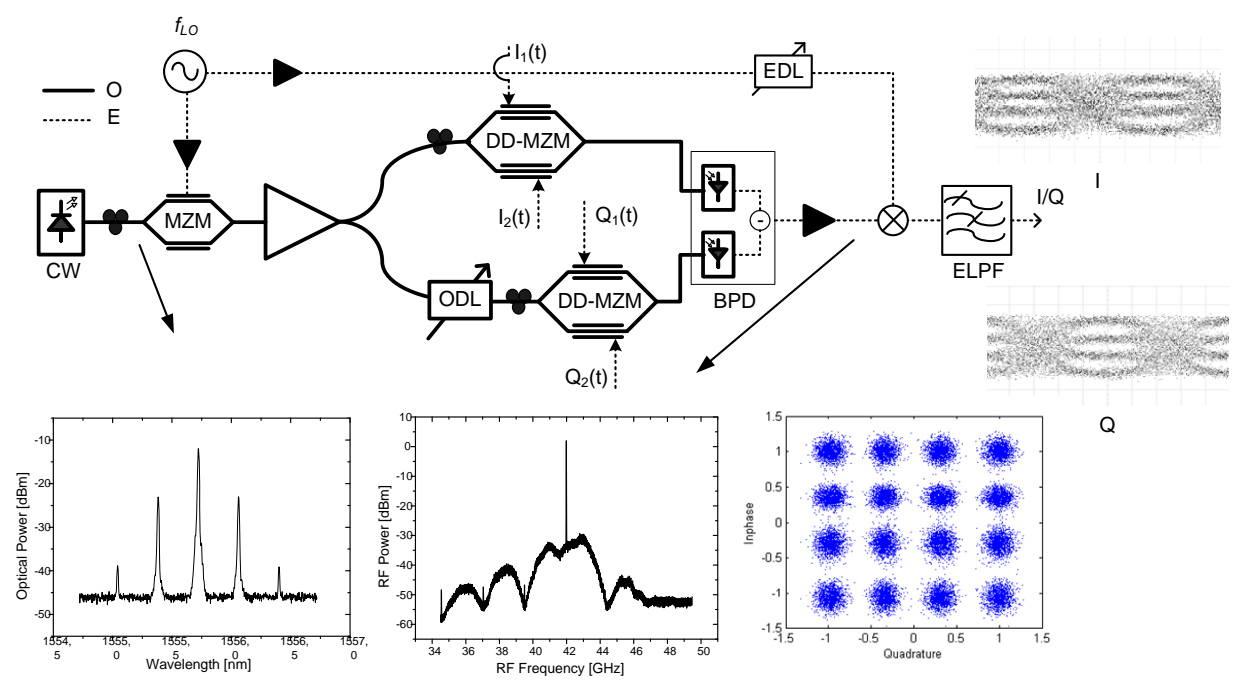

Figure 3.11: Overview of the experimental set-up and results obtained of a 10 $\mathrm{Gb} / \mathrm{s}$ 16-QAM signal generation using photonic vector modulator.

on an optical carrier using an optical coupler, the LO modulated optical signal is divided into two arms. One of the arm is optically delayed using an optical delay line, to satisfy the quadrature condition for QAM modulation. Using external MZ modulators, the electrical four level $I$ and $Q$ signals (4-ASK) are modulated on the two arms and photo-detected in a balanced photo-detector, whose output is the $10 \mathrm{~Gb} / \mathrm{s} 16-\mathrm{QAM}$ wireless signals. The advantage of this technique is that only one optical source is used which reduces the noise in the system, and the use of balanced PD cancels the common optical DC power, which reduces the residual LO peak. From the RF spectrum as shown in fig. 3.11 the $10 \mathrm{~Gb} / \mathrm{s} 16-$ QAM wireless signals occupy a bandwidth of around $4 \mathrm{GHz}$, resulting in a spectral efficiency of around $2.66 \mathrm{~b} / \mathrm{s} / \mathrm{Hz}$.

\subsection{Distribution of Wireless Signals in a Radio- Over-Fibre Access Network}

The increase in the capacity demand is pushing the use of high $\mathrm{mmW}$ frequencies, which requires more BSs to cover a wider geographical area. As 
discussed, the cost-effective solution is to have a centralised processing and signal distribution to the BSs. Depending on the services, the mmW signals can be either distributed in a broadcast scenario [102], or each BS can be sent a different service. WDM technology offers a good solution, where each BS can be linked to a specific wavelength [103, 104]. Several techniques were considered for a WDM ROF network, like using optical interleaved DWDM sources with different channel spacing [105 107], super-continuum sources 108, 109], etc. For multiplexing and demultiplexing, components like fibre Bragg's gratings (FBG) 103 and arrayed waveguide gratings (AWGs) [106 are used. Also several WDM network configurations have been demonstrated, where multi service transmission and simultaneous wireless and baseband signal transmission was incorporated 110112 .

WDM based RoF networks are being investigated for distribution of wireless services. Most of the traffic in wireless networks changes dynamically mainly due to the mobility of the users. The WDM configuration is a key physical layer technology that enables wireless signal distribution, but to take the full advantage of the optical network the dynamic changes of wireless services traffic should be incorporated into the physical layer. Dynamic bandwidth allocation is one way to solve the traffic changes, but dynamic channel allocation would take the full advantage of the optical domain. Dynamic channel allocation can be implemented in a WDM network using a wavelength switching/routing mechanism, where channels are dynamically allocated to requesting BSs [113. Wavelength allocation using wavelength routers use a large number of components increasing the cost, whereas a compact alternative based on a fold-back arrayed waveguide grating (AWG) incorporating a single optical switch (OS) has been recently proposed for the down and uplink access with a significantly reduced number of components 114 .

\subsubsection{Dynamic Reconfigurable Optical WDM Network}

In paper $\mathrm{H}$ a dynamically reconfigurable optical WDM network based on an AWG and OS, similar to the configuration in 114 is presented. The transmission of PVM generated wireless signals over such a network is performed to demonstrate the ability of a PVM systems to co-exist with 


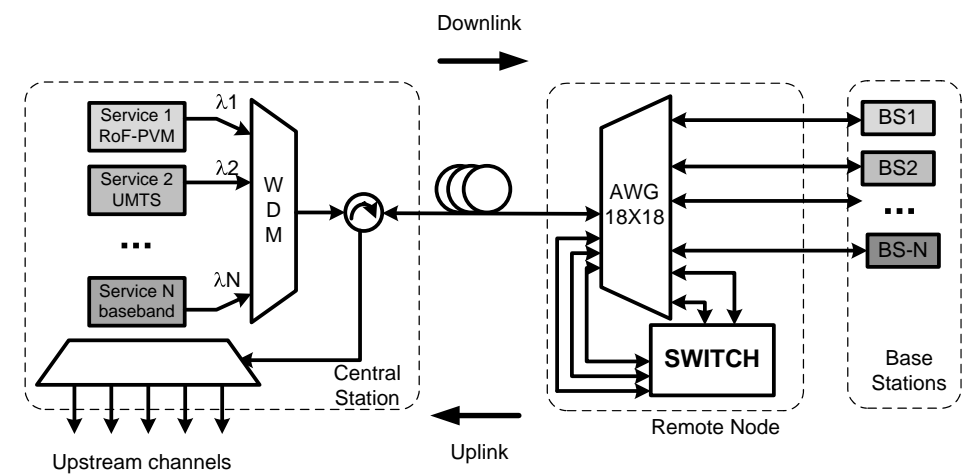

Figure 3.12: Schematic of a dynamically reconfigurable optical WDM network for RoF access.

optical access networks based on WDM. Figure 3.12 shows the schematic of the reconfigurable optical network. At the CS multiple services are generated and are allocated wavelengths to be transmitted to the remote node (RN). At the RN, the channels received from the CS are routed directly to the BSs which have assigned channels. These pre-defined services to the BSs are named as "own channels" which guarantee service to the BSs. The remaining "extra channels" are fed back into the optical switch and the AWG, which depending on the requirement from the BSs are routed to the demanding BS as extra channels in a cyclic manner. Hence each BS has a own channel and several extra channels depending on the channel allocation. To characterize the behaviour of photonic vector modulated signals, a $1.25 \mathrm{~Gb} / \mathrm{s}$ QPSK signal was generated using a PVM architecture (fig. 3.2(b)) at a carrier frequency of $10 \mathrm{GHz}$. The PVM signals were generated using a total optical bandwidth of $0.42 \mathrm{~nm}$ (centred at $1548.8 \mathrm{~nm}$ ) to comply with the $50 \mathrm{GHz}$ DWDM spacing of the AWG. The PVM generated QPSK signals from the CS were transmitted to the RN using a $10 \mathrm{~km}$ SMF. At the remote node, the QPSK wireless signals were routed to two BS: BS1 and BS2, as both own and extra channels. To analyse the quality of the PVM signals at the BSs, Q-factors were measured by varying the PD input power and plotted in fig. 3.13 . Also as a comparison ASK signals were generated simply by switching off one of the laser in PVM architecture. It can be seen that at both the BSs the QPSK signals have the same performance, but when routed as extra channels, power penalty is induced. This is because when the signals are routed as extra channels, they are passed through the optical switch and 


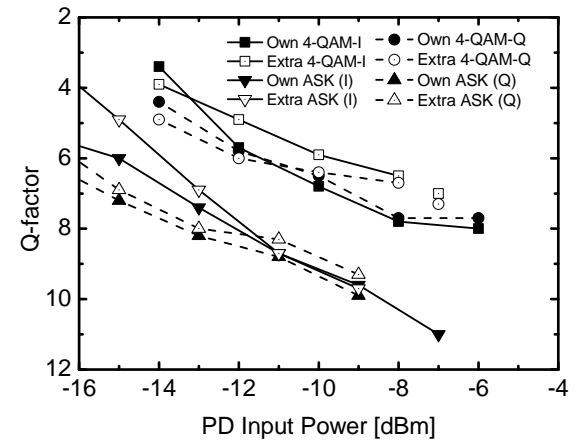

(a)

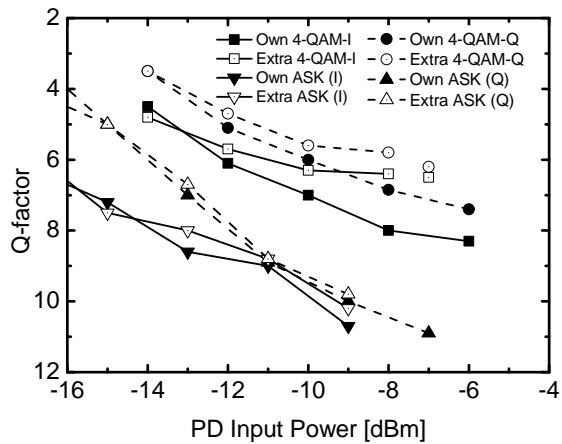

(b)

Figure 3.13: The variation of Q-factor of the PVM generated QPSK signals and reference ASK signals with respect to the PD input power at BS1 (a) and BS2 (b) for own and extra channels assignments.

AWG again which induces extra $5 \mathrm{~dB}$ of optical losses and passing the same optical signals twice through an AWG results in narrow filtering of the signals. The results shows error free performance in both own and extra channel routing.

\subsubsection{Multi service Co-existence [Paper $\mathbf{H}]$}

To the above mentioned network architecture more services were added. The PVM generated QPSK signals were assigned as own channel to BS1, and a UMTS signal was modulated on a $1549.6 \mathrm{~nm}$ optical carrier and assigned to BS2 as own channel. A third extra channel was generated at the CS which is a $155 \mathrm{Mb} / \mathrm{s}$ baseband data on a $1551.2 \mathrm{~nm}$ optical carrier and routed as extra channel to either BS1 or BS2. Very little power penalty is induced on the QPSK signals in the presence of the extra channel in BS1 or BS2. Similarly the quality of the UMTS signal was measured at BS2 and the error vector magnitude was measured and the results indicate the compliance of the quality with the 3GPP standard of EVM less than 1.2\% .The co-existence of PVM with UMTS and $155 \mathrm{Mb} / \mathrm{s}$ does not influence the quality of the latter signals in any way which demonstrate the robustness of the reconfigurable optical network. The degradation in the QPSK signals is mainly due to the vector 


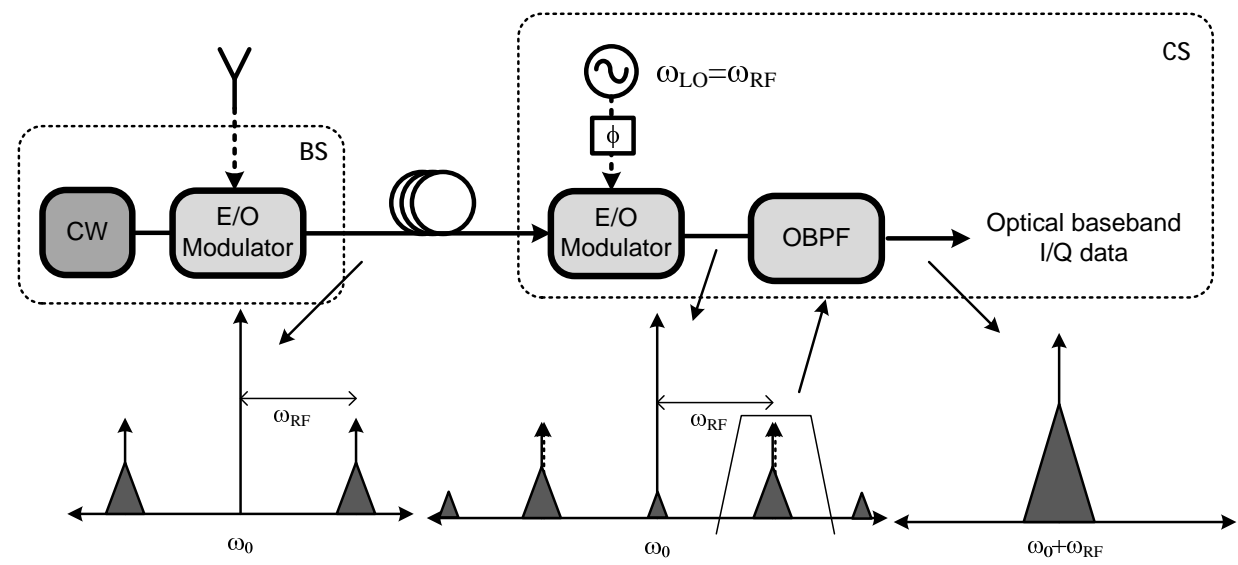

Figure 3.14: Illustration of working principle of a photonic vector demodulator.

modulation and demodulation processes and the fibre's chromatic dispersion induced power fading. The remote node, including the optical switch and the co-existence of other services in the WDM network add very little degradation. It has been demonstrated that a dynamically reconfigurable optical network is compatible with various RoF and digital signals in a co-existence scenario, reducing the base station complexity, and increasing the efficiency network's capacity usage.

\subsection{Photonic Vector Demodulation: Working Prin- ciple}

In the previous section, photonic techniques for generation of spectral efficient vector modulated wireless signals is presented for RoF downlinks. The wireless signals transmitted over air are received at another BS, which are transmitted over a RoF uplink to a CS to be demodulated. When the wireless uplink signals are photo-detected at the BS, the fibre's chromatic dispersion induces RF power fading limits the transmission and performance of the signals as seen in chapter 2. To mitigate this effect, several techniques have been proposed, and in this section a direct demodulation of RF wireless signals using photonic techniques is presented, 
which is tolerant to fibre's chromatic dispersion. The proposed technique directly converts a wireless QAM signal into its respective in-phase and quadrature optical baseband components without the need of an electrical demodulator. Figure 3.14 shows the concept behind a photonic vector demodulator.

In a RoF uplink, the wireless signals received at the antenna BS are modulated on an optical carrier using a DSB modulation in an external modulator, and are transmitted to the CS over a SMF. At the CS, to perform demodulation of the vectorial wireless signals, the optical DSB signal is modulated with a local oscillator which is tuned exactly to the received RF signal frequency. The optical carrier of the DSB signal generates the fundamental harmonics by the LO tone, which superimpose on the side-bands created by the wireless signals. By tuning the phase on the LO signal, the in-phase and quadrature components can be obtained in the optical domain on one of the side-bands as seen in fig. 3.14 The SMF dispersion induced phase shift is seen on the side-band, which only converts to RF power fading, when both the optical carrier and the side-bands are photo-detected. But in this demodulation technique, one of the side-bands is filtered, and photo-detected using a low frequency (baud-rate) $\mathrm{PD}$, no RF power fading is present.

The expression of the filtered side-band (see Appendix A) can be expressed as:

$$
\begin{aligned}
E_{\text {out }}^{U S B}(t)= & \sqrt{P} T_{f f} \exp j\left(\omega_{0} t+\omega_{R F}+\phi_{0}\right) \\
& {\left[J_{0}\left(m_{R F}\right) J_{1}\left(m_{L O}\right) \exp j\left(\phi_{L O}\right)\right.} \\
& \left.+J_{0}\left(m_{L O} J_{1}\left(m_{R F}\right) \exp j\left(\phi_{R F}+\Psi+\frac{\beta_{2} L \omega_{R F}^{2}}{2}\right)\right)\right]
\end{aligned}
$$

To demodulate the in-phase component, the phase of the LO and the RF signal should be matched according to:

$$
\phi_{L O}=\phi_{R F}+\frac{\beta_{2} L \omega_{R F}^{2}}{2}
$$

Thus the photo-current of the in-phase component is:

$$
i_{P D}^{I}(t) \approx J_{0}\left(m_{L O}\right) J_{1}\left(m_{L O}\right) J_{0}\left(m_{R F}\right) J_{1}\left(m_{R F}\right) I(t)
$$


To demodulate the quadrature component, the phase relation between $\mathrm{LO}$ and RF signal is:

$$
\phi_{L O}=\phi_{R F}+\frac{\beta_{2} L \omega_{R F}^{2}}{2}+\frac{\pi}{2}
$$

Thus, the quadrature photo-current is:

$$
i_{P D}^{Q}(t) \approx J_{0}\left(m_{L O}\right) J_{1}\left(m_{L O}\right) J_{0}\left(m_{R F}\right) J_{1}\left(m_{R F}\right) Q(t)
$$

\subsubsection{Performance Optimization}

From the photo-currents of the demodulated signals, it can be deducted that the main parameters that determine the performance of the demodulator are the RF and LO modulation indices. The performance of a MZM was theoretically analysed in chapter 2 and fig. 2.6(a) shows that the optimum modulation index for maximum performance is around $\pi / 2$. To analyse the performance of the demodulation technique a $60 \mathrm{GHz} 10$ $\mathrm{Gb} / \mathrm{s}$ QPSK wireless signal with 4-QAM/QPSK modulation was simulated using a commercial optical simulator. The modulation indices of both the RF modulator and LO modulator were varied and plotted in fig. 3.15. Later, after attaining the optimum values for the modulation indices, the values were fed into the system and to analyse the performance of the demodulator, BER was measured using an optical pre-amplified receiver. The BER was first calculated using a back-to-back scenario and later using a $20 \mathrm{~km}$ of SMF between the two modulators, and plotted as shown in fig. 3.15. From the plots shown in fig. 3.15 it can be seen that the modulation indices of both the modulators must be well chosen by maximizing the quality of the signal. From the BER plots it is seen that the receiver sensitivity of the demodulator indicates a good performance and that after transmitting the $60 \mathrm{GHz} 10 \mathrm{~Gb} / \mathrm{s}$ wireless signals over $20 \mathrm{~km}$ of SMF very little power penalty is induced. The BER results prove that the demodulating PvDM RoF uplink technique is immune to the fibre's chromatic dispersion induced RF power fading, unlike the conventional RoF uplinks. 


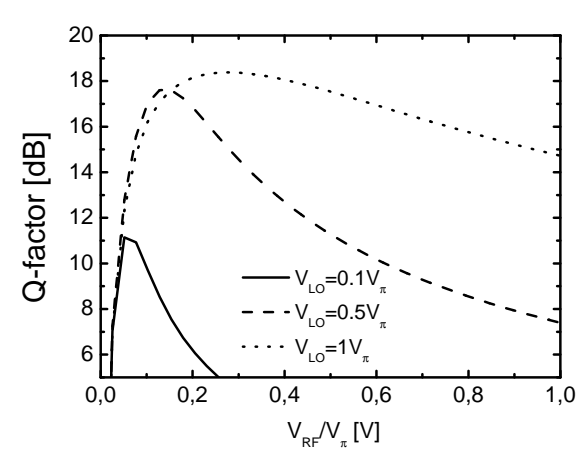

(a)

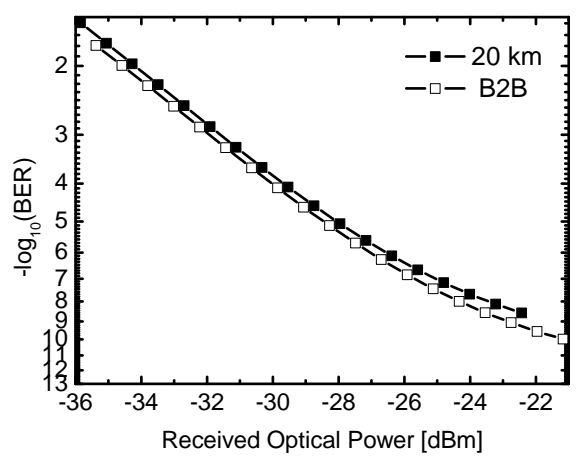

(b)

Figure 3.15: Simulated results of a $60 \mathrm{GHz} 10 \mathrm{~Gb} / \mathrm{s}$ QPSK wireless photonic vector demodulating uplink. The variation of $\mathrm{Q}$-factor with the RF modulation index (a) and the calculated BER in back-to-back and after $20 \mathrm{~km}$ of SMF (b).

\subsubsection{Architectures for PvDM [Paper I]}

Photonic vector demodulation can be easily integrated in existing RoF uplinks. Figure 3.16 shows a typical RoF link with a bi-directional transmission for both downstream and demodulating upstream. The optical carrier for carrying the upstream data is sent from the CS to the BS. At the BS, the optical carrier assigned for carrying the upstream signals is passed through an optical modulator where the received wireless signals are modulated. The RF modulated optical carrier is fed into the optical fibre again where at the CS the upstream signals are demodulated using the PVdM technique. Using this configuration the BS are kept simple without the need of optical sources or RF demodulators.

Photonic vector demodulation can be performed in different ways, depending on the system design. Figure fig. 3.17 shows the different architectures for performing PVdM. In the first architecture, only one optical carrier is used where after modulating the RF signals, the optical signal is split into two parts and demodulated independently for in-phase and quadrature. Instead of using one optical carriers two separate optical carriers at different wavelengths $\left(\lambda_{1}, \lambda_{2}\right)$ can be used to independently demodulate the in-phase and quadrature on $\lambda_{1}$ and $\lambda_{2}$ respectively. The advantages of using two optical carriers instead of one are higher opti- 


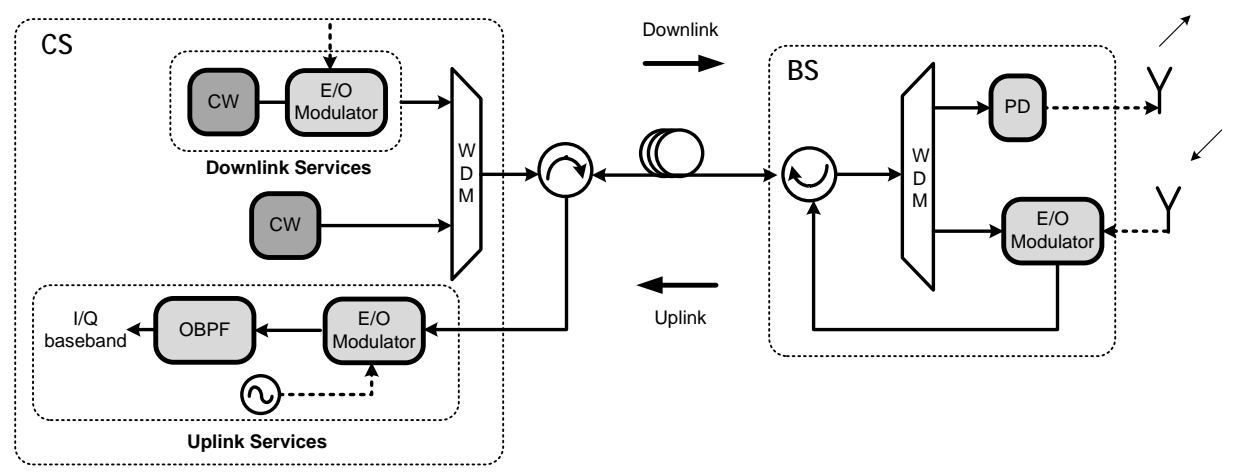

Figure 3.16: Illustration of a RoF bi-directional link with direct photonic vector demodulation.

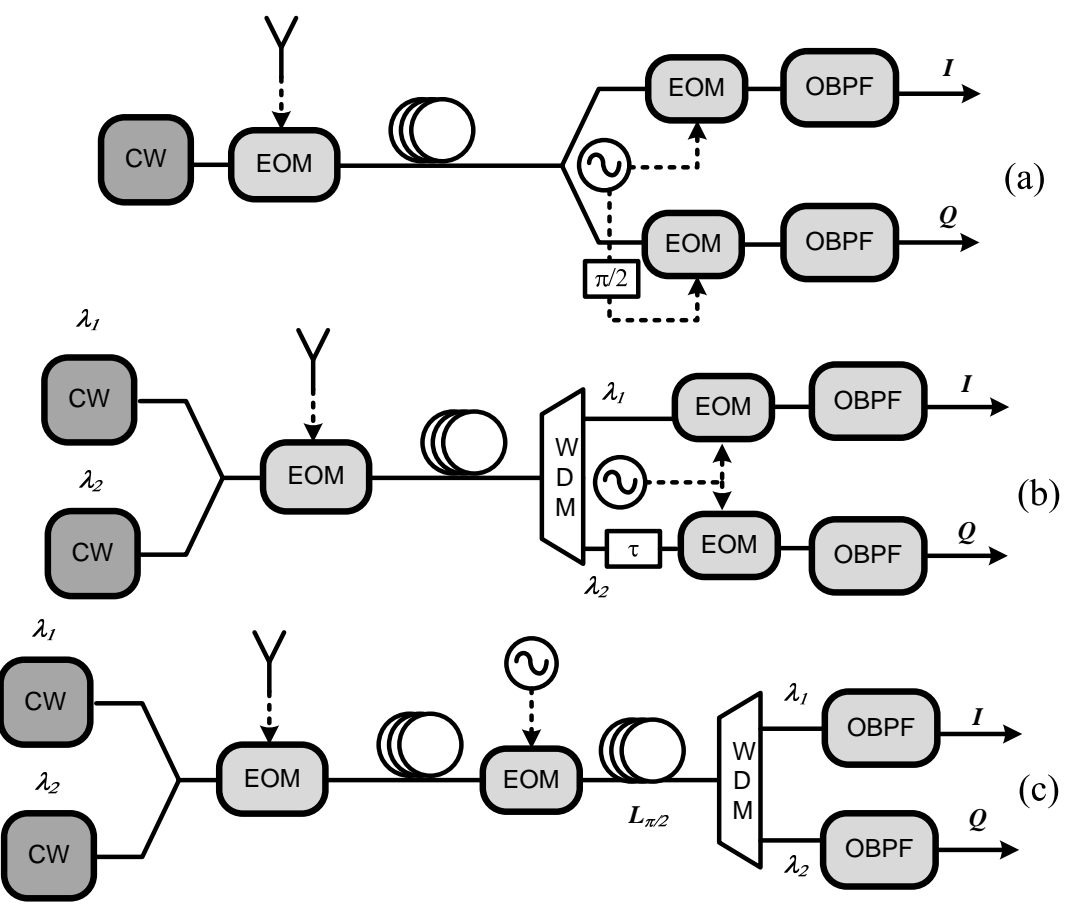

Figure 3.17: Schematics of different architectures for photonic vector demodulation, using single optical carrier (a), using two optical carriers and optical delay line based RF phase shifter (b), and using two optical carriers and dispersion based RF phase shifter (c). 
cal power budget, use of optical phase shifting configuration common to PVM like optical delay or dispersion based phase shifting.

In paper I, the PvDM architecture shown in fig. 3.17(b) is experimentally demonstrated where a $2.5 \mathrm{~Gb} / \mathrm{s} 20 \mathrm{GHz}$ QPSK wireless signal was successfully demodulated. The quality of the demodulation process was quantified by measuring the BER of both in-phase and quadrature components in an optical pre-amplified receiver similar to the simulations described earlier. Error free demodulation was performed where a receiver sensitivity of $-27 \mathrm{dBm}$ was measured at a BER of $10^{-9}$, indicating a very good performance.

\subsection{Conclusion}

Generation of spectral efficient quadrature amplitude modulated wireless signals using baseband optical modulation in combination with RF upconversion techniques was presented in this chapter. The photonic vector modulation technique is able to generate wireless signals with high quality and experimental demonstration of $10 \mathrm{~Gb} / \mathrm{s}$ wireless links was performed in the $60 \mathrm{GHz}$ band. Using PVM architectures wireless signals with SNR as high as $40 \mathrm{~dB}$ can be generated as long as low RIN coefficient lasers are incorporated. Also the potential of PVM architectures for generation of higher spectral efficient modulation formats like 16-QAM was studied and experimentally demonstrated where a $10 \mathrm{~Gb} / \mathrm{s}$ 16-QAM signal generation with a spectral efficiency of $2.66 \mathrm{~b} / \mathrm{s} / \mathrm{Hz}$ has been successfully demonstrated.

The crucial parameters that determine the quality of the generated signal are the data modulation extinction ratio and the RIN coefficient of the lasers. Direct modulation of lasers offer a cost-effective solution for implementing the PVM architectures but suffer from lower extinction ratio. The lower extinction ratio is not a limitation in robust modulation formats like QPSK, but if higher order modulation formats are to be implemented external modulators offer a better performance. It should be noted that the increase in the extinction ratio only favours in a low RIN scenarios, where RIN parameters below $-145 \mathrm{~dB} / \mathrm{Hz}$ are required. The 
strict requirement of laser RIN coefficient is due to the IM/DD nature of the up-conversion technique used which is a drawback of the PVM architecture.

PVM generated wireless signals were transmitted in a dynamically reconfigurable optical network proving the ability of the PVM architectures' compliance with current network architectures. It was noticed that PVM generated signals can co-exist in a multi-service distribution scenario in an optical access network, and can be dynamically routed to demanding BSs with additional penalty.

The drawbacks of PVM of wireless signal generation is the use of conventional up-conversion techniques using external modulators with DSB modulation, which is prone to fibre's chromatic dispersion induced RF power fading. The dependency of the RF power fading on the length and the RF carrier frequency limits the performance at higher frequencies. The dispersion can be compensated either using dispersion compensating modules at the CS, or choosing the fibre length to maximize the RF power. Suppressed carrier modulation do not suffer from dispersion induced power fading, but the delay between the spectral components contribute towards jitter of the wireless signals which will limit the performance.

Similarly, RoF uplink architecture is proposed with direct photonic vector demodulation capability. The PVdM architecture is immune to fibre's chromatic dispersion induced RF power fading, which is due to the sideband filtering of the RoF signal. The ability of directly converting a wireless QAM signal into its respective baseband in-phase and quadrature components has the added advantage since no RF or IF demodulators are required, and the optical baseband components can be directly fed into another optical networks, extending the reach of access networks. One downside of the demodulation technique is the requirement of electrical phase locked loop, which are complicated to implement at higher $\mathrm{mmW}$ frequencies. 
Chapter 4

\section{Optical Baseband Modulation for Millimetre Wave Wireless}

\subsection{Introduction}

In the previous chapter, several radio over fibre techniques for generation and demodulation of $\mathrm{Gb} / \mathrm{s}$ wireless links were described. All the radio over fibre techniques in the previous chapter were limited by the fibre's chromatic dispersion or require complex $\mathrm{mmW}$ electronic circuitry like phase locked loops and LO signal generator. Photonic vector modulation technique as seen in previous chapter is based on IM/DD technique for up-conversion, where the baseband data and the LO signal is intensity modulation and photo-detected directly. The disadvantage of IM/DD scheme in PVM architectures is the use of amplitude modulation of inphase and quadrature components for the vector signal generation which has a non-zero average optical power. This residual average optical power results in a high LO peak of the $\mathrm{mmW}$ signal which is a a limiting factor. Also the up-conversion techniques involved are power inefficient, which limits the total power of the RF signal generated. Instead of amplitude modulation of the optical signal, if phase modulation is used, there will 
be no LO peak in the RF signal. But phase modulation optical signals cannot be up-converted using double side-band modulation since at the $\mathrm{PD}$ only the optical intensity is converted to electrical domain.

Phase modulated $\mathrm{mmW}$ signal generation attracted very little interest and Doi et al, 115 demonstrated a modulator concept based on delay modulation to generate phase shift keying (PSK) modulation wireless signals. Another way of generating phase modulated wireless signals is using single side-band modulation, where two optical carriers are required to generate the RF signal, but only one of the optical carriers is phase modulated. When such a SSB signal is photo-detected, the optical phase modulation is converted into a $\mathrm{mmW}$ phase modulated signal. Optical phase modulated communication links are growing rapidly with mature technology, and the use of optical baseband phase modulation for wireless links can have several advantages. The wireless links which will be based on optical modulation formats can provide a great level of integration between the optical and wireless networks and help reduce the cost by sharing the equipment.

In this chapter, the integration of optical baseband modulation and demodulation techniques into wireless applications in a RoF scenario is discussed. In section 4.2, the recent advances in optical baseband modulation formats are described. In section 4.3, an integrated optical modulator concept for generation of wireless signals using optical baseband modulation formats is described. In section 4.4 the use of optical coherent detection in combination with digital signal processing and its use for demodulation of wireless signals is discussed. In section 4.5, a novel technique based on heterodyne mixing of two optical carriers for wireless signal generation is presented followed by conclusions in section 4.6.

\subsection{Optical Baseband Modulation Formats}

From the beginning of optical communications till recently NRZ-OOK (non return to zero on-off-keying) has been the modulation format for most commercial applications. NRZ-OOK is amplitude modulation of the optical source, which is performed either switching ON or OFF the 
laser in a direct modulated laser or using external modulators. OOK modulation format in general has poor non-linearity tolerance because of the peak optical carrier. In contrary optical phase shift keyed (PSK) format carries the information in the optical phase, and the optical spectrum in fig. 4.1 shows no peak. One of the basic advantages of PSK over OOK format is that PSK formats the symbol distance is $\sqrt{2}$ times higher than OOK for the same average optical power [116], which translates to $3 \mathrm{~dB}$ lower OSNR (optical signal to noise ratio) requirement for PSK formats. Phase modulated optical signals cannot be directly

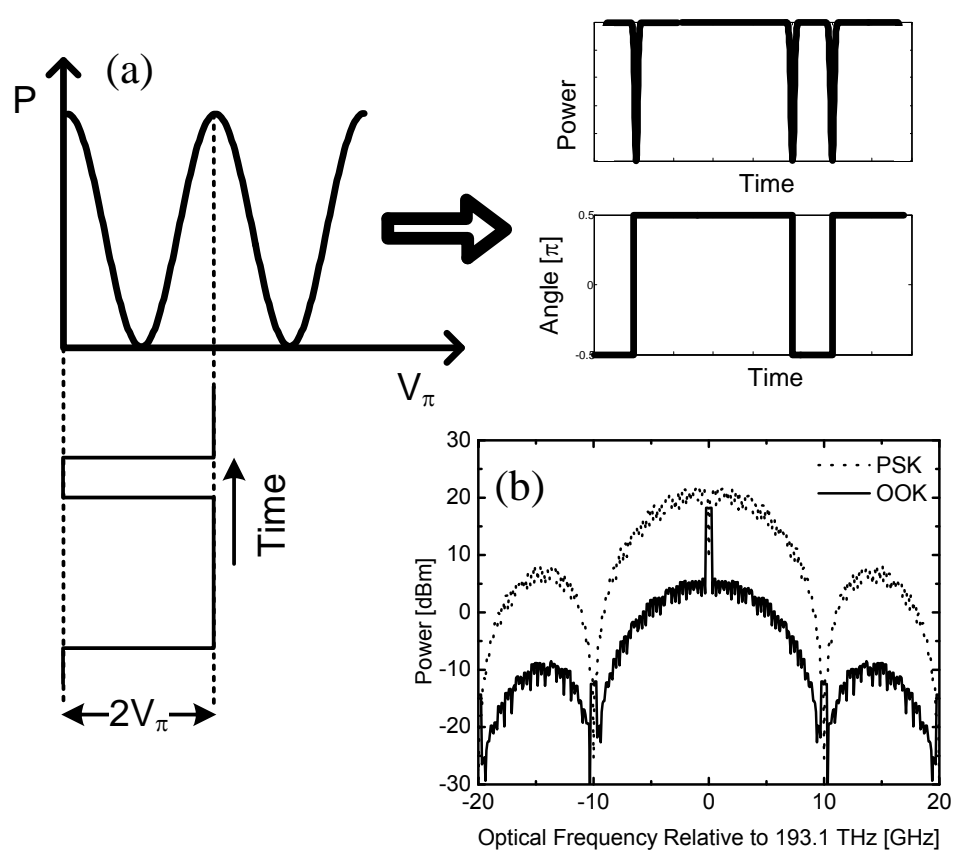

Figure 4.1: Illustration of working principle of a Mach-Zehnder modulator for generating phase modulation (a), and the optical spectra of OOK and PSK signals (b).

detected since the PD only detects the optical power, hence phase to amplitude demodulation must be performed before photo-detection. Demodulation of PSK signals can be performed by mixing the incoming signal with another optical carrier at the receiver, the photo-current will depend on the phase difference between the two optical carriers. These systems using homo-dyne detection are called coherent systems [117] and 
have attracted a lot of interest in the early 90s for record receiver sensitivities. The early coherent systems required some kind of phase locking between the incoming optical signal and the optical local oscillator. Most of the locking techniques use optical phase locked loops [118, 119, which required low linewidth lasers and lower loop delays. Later, demodulation of PSK signal using self homo-dyne technique in combination with differential encoding is used [120] where a 1-bit delay Mach-Zehnder interferometer is used to demodulate the differential encoded PSK signals, also named as DPSK. DPSK based optical communication systems have been extensively investigated with record breaking capacities [121, 122].

Modulation of the optical phase can be performed using a phase modulator which will result in a constant amplitude, whereas due to the instantaneous phase shift between 0 and $\pi$ residual chirp is introduced in the bit transitions. The practical (D)PSK links use a MZM biased at the minimum transmission point. Every bit transition results in passing through the minimum transmission point, which introduces a $\pi$ phase shift as depicted in the fig. 4.1 .

\subsubsection{DPSK Demodulation}

In a DPSK receiver as shown in fig. 4.2, the differentially encoded phase information should be decoded and converted into amplitude before photodetection. This is done using a 1-bit delay Mach-Zehnder interferometer (DMZI) which demodulates the phase difference between every bit and its successor, which contains the differential coding. The DMZI splits the input signal into two arms, where one arm is phase shifted by $\pi$ and the other arm delay by $\tau=T$ where $\mathrm{T}$ is the bit-period. The two optical signals are combined and split again. The phase difference $\phi$ for DPSK is ideally 0 or $\pi$ and depending on the phase shift either constructive or destructive interference occurs. Figure shows the working principle and the optical spectra at the output of the two ports. It can be seen that even though the optical spectrum for the constructive and destructive ports is different, they carry the same information but inverted in one arm. When balanced photo-detection is applied the resulting data signal has a 3-dB higher receiver sensitivity compared to a single ended detection 123 . 


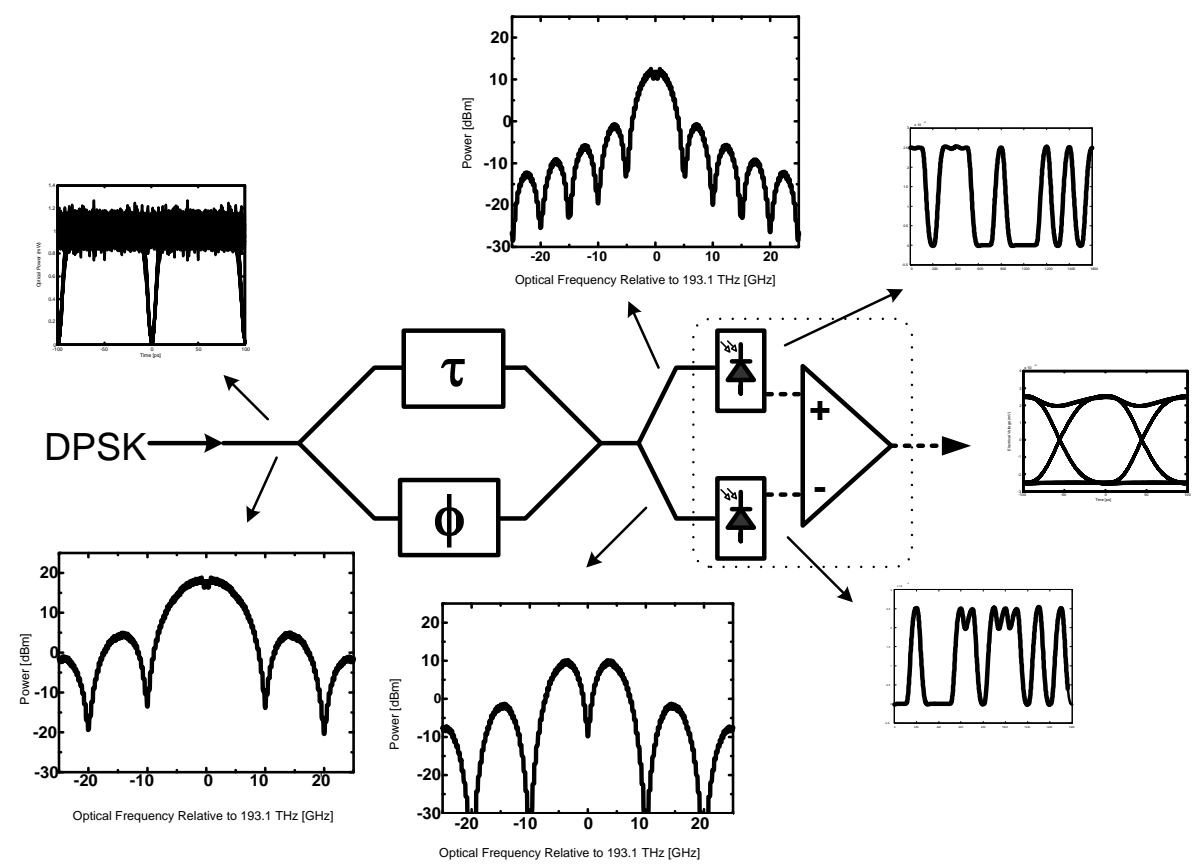

Figure 4.2: Schematic of a 1-bit delay Mach-Zehnder interferometer and its use for detection of differential phase shift keyed optical signals.

\subsubsection{Optical (D)QPSK}

Quadrature phase shift keying is a four level phase modulation where the information is encoded using one of the four phase levels. Early QPSK optical communication systems were based on coherent detection, with both homo-dyne and heterodyne techniques were investigated [124]. The robustness of DPSK transmission links to the fibre impairments has attracted a lot of interest in differential quadrature phase shift keying, [125 127], where several demonstrations of long haul systems were made. DQPSK is the choice of modulation format for $40 \mathrm{~Gb} / \mathrm{s}$ long haul systems, and the low baud rate of the DQPSK modulation format has attracted a lot of research efforts for $100 \mathrm{~Gb} / \mathrm{s}$ transmission systems 128130 .

Four level phase shift keying occupies half the bandwidth compared to 2PSK modulation for the same bit-rate. (D)QPSK constellation evidently 


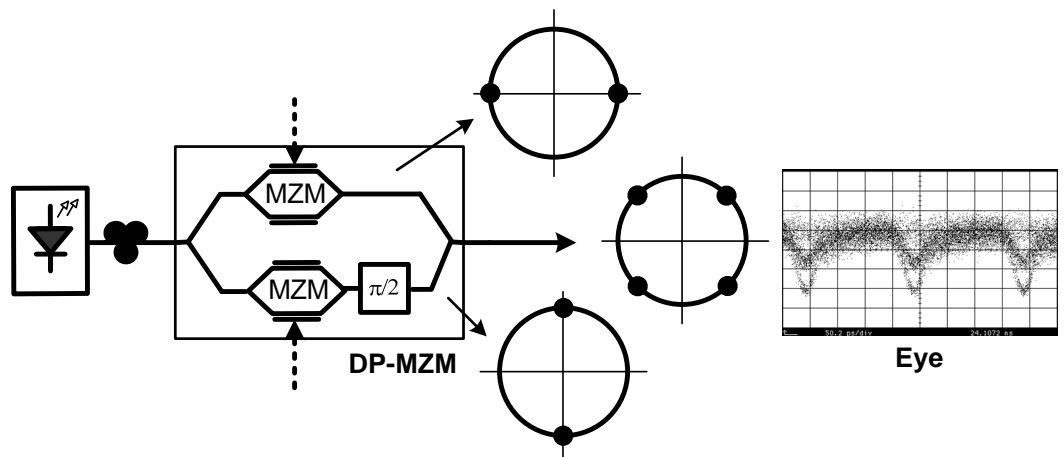

Figure 4.3: Schematic of an optical dual parallel Mach-Zehnder modulator and the resulting constellation and eye diagram.

have four constellation points and the distance between the symbols is halved compared to (D)PSK. The reduced distance between the symbols in constellation would require a $3 \mathrm{~dB}$ higher OSNR for (D)QPSK compared to (D)PSK at a given receiver sensitivity. But for the same bit-rate (D)QPSK has half the optical bandwidth, requiring the same OSNR for (D)QPSK and (D)PSK. The generation of four level phase modulation can be performed using various modulator configurations [131-133], but the most common modulator configuration is the use of dual parallel Mach-Zehnder modulator (DP-MZM) [131] also referred as I/Q MZM. Figure 4.3 shows the schematic of the modulator for, and the symbol allocation of, a (D)QPSK optical signal. Higher spectrally efficient modulation formats like quadrature amplitude modulation (M-QAM) can be generated using the I/Q MZM, which are attracting research interest for $100 \mathrm{~Gb} / \mathrm{s}$ links [134.135.

Using the DP-MZM, two (D)PSK signals are generated by two independent data streams $(I, Q)$ in the two MZMs, and one of the arms is phase shifted using a $\frac{\pi}{2}$ phase shifter before both the signals are combined. The combined optical signal is a four-level phase modulated signal with phase values of $\left\{\frac{\pi}{4}, \frac{3 \pi}{4}, \frac{5 \pi}{4}, \frac{7 \pi}{4}\right\}$. At the bit transitions when both $I$ and $Q$ are zero, the total optical power drops to zero, whereas when either one of them is zero, the optical power drops to half, which is seen from the three-level eye diagram from fig. 4.3 .

For differential quadrature phase shift keying (DQPSK) a pre-coding 


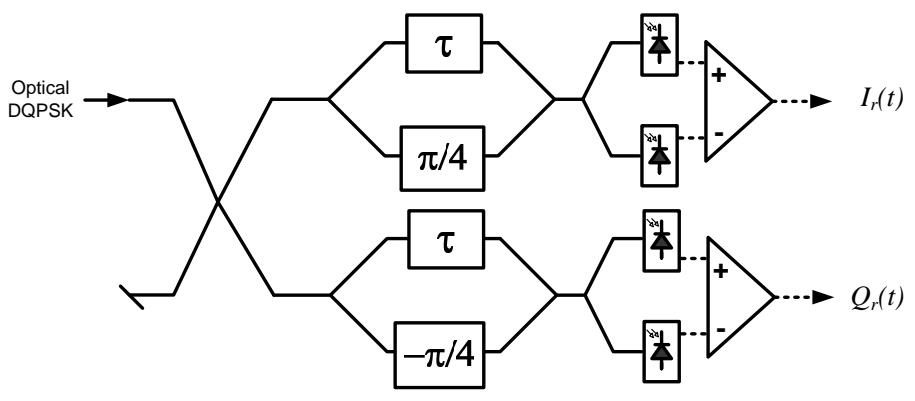

Figure 4.4: Delay demodulation based receiver for optical DQPSK signals.

should be implemented for a self-homodyne based detection to correctly decode the phase modulated signals. The differentially coded signals are demodulated using a 1-bit delay interferometer (DMZI) with a phase offset of $\pm \frac{\pi}{4}$. The demodulated photo currents of the DQPSK receiver with balanced photo-detection (fig. 4.4 )can be expressed as:

$$
\begin{aligned}
I_{r}\left(t_{k}\right) & =-\mathfrak{R} \frac{E^{2}}{2}\left[\cos \left(\Delta \phi_{k}\right)-\sin \left(\Delta \phi_{k}\right)\right] \\
Q_{r}\left(t_{k}\right) & =-\mathfrak{R} \frac{E^{2}}{2}\left[\cos \left(\Delta \phi_{k}\right)+\sin \left(\Delta \phi_{k}\right)\right]
\end{aligned}
$$

Where $\delta \phi_{k}=\phi_{k+1}-\phi_{k}$ is the phase difference between two adjacent symbols.

Depending on the phase offset of the DZMI the $I$ and $Q$ components can be detected independently.

\subsection{Integrated Millimetre Wave Modulator Con- cept}

The generation of wireless signals based on optical phase modulation can be performed by implementing a SSB configuration. In this SSB signal format, two synchronous optical carriers are generated where only one of the optical carriers is phase modulated. One way of doing this is to filter one of the optical signals and pass it through an optical modulator, 
and the combined signal will be SSB in nature as shown in fig. 4.5. Several experimental demonstrations have been reported on the use of this technique [136 138] using common optical components like fibre Bragg's gratings and optical modulators like MZM. For phase modulation, the use of common components require that the two optical signals have phase and polarization correlation prior to photo-detection. This can be very difficult to implement since the optical carriers travel different paths, and for multi-level phase modulation the requirements on phase correlation increases.

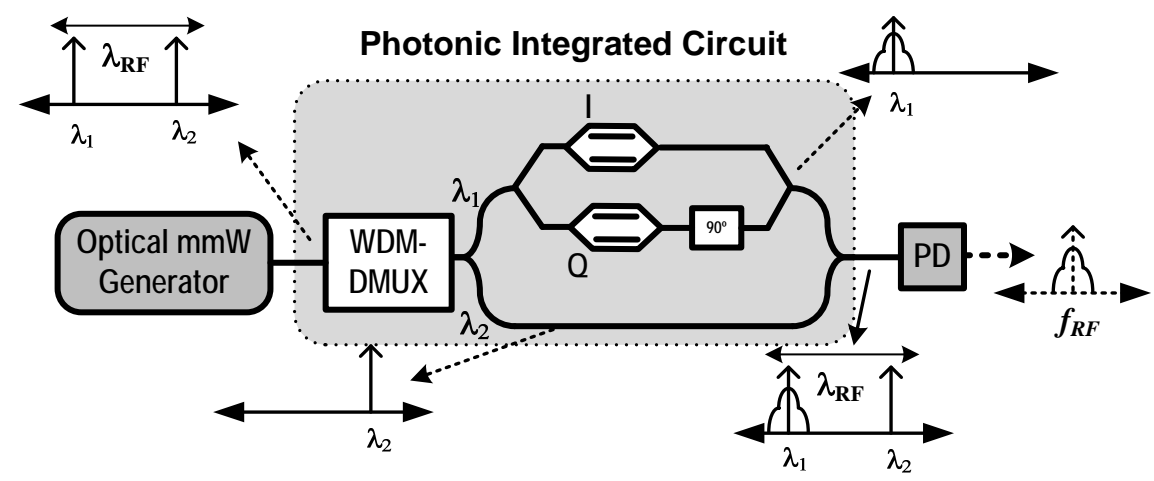

Figure 4.5: Illustration of a millimetre wave I/Q optical modulator.

Photonic integration of the optical modulator with the filters can provide the required phase and polarization control since the path lengths can be accurately controlled and the devices can de designed to maintain polarization. Optical devices are mostly made using III-V semiconductors, but recently silicon based optical devices has attracted a lot of interest for both digital and analogue applications 139 145]. The main advantage of silicon photonics technology is the possibility of monolithic integration of photonics and electronics using cost-effective CMOS technology 141. Optical modulators, photo-detectors, filters, and other optical components using Si have been demonstrated for FTTH networks aimed at reducing the cost [146]. Silicon based optical modulators are being extensively investigated 139 142, 147, 148], and modulator speeds of up to $40 \mathrm{~Gb} / \mathrm{s}$ [147] have been demonstrated. For the application in discussion, silicon photonics offer very good solution by integrating the filter and the optical modulators, and Si based MZM have been demonstrated [139 
which can be used for generating phase modulation.

\subsubsection{Device Parameter Requirements}

Though Silicon photonics offer several advantages, the technology is still in development and the performance of the devices needs to be improved. The main limitation of the Silicon technology is its poor electro-optic coefficient, which limits the efficiency of the electro-optic effect. The Si based modulators and filters have low extinction ratio which will affect the over performance of the signal generator.

The optical field of a dual wavelength coherent light source can be expressed as:

$$
E_{0}(t)=\sqrt{2 P_{i n}}\left[\exp j\left(\omega_{1} t\right)+\exp j\left(\omega_{2} t\right)\right]
$$

Where $\omega_{1}, \omega_{2}$ are the frequencies of the optical carriers. The two optical carriers are demultiplexed into two fields, $E_{1}(t)$ and $E_{2}(t)$ using a finite extinction ratio $(\alpha)$ WDM-DMUX.

$$
\begin{aligned}
& E_{1}(t)=\sqrt{P_{\text {in }}}\left[\alpha \exp j\left(\omega_{1} t+\phi(t)\right)+(1-\alpha) \exp j\left(\omega_{2} t\right)\right] \\
& E_{2}(t)=\sqrt{P_{\text {in }}}\left[(1-\alpha) \exp j\left(\omega_{1} t+\phi(t)\right)+\alpha \exp j\left(\omega_{2} t\right)\right]
\end{aligned}
$$

Modulating the optical phase of the carrier $E_{1}(t)$ and combining the modulated optical signal with the unmodulated optical carrier using a asymmetric coupler (coupling ratio $\beta$ ), the output can be expressed as:

$$
\begin{aligned}
E(t)= & \sqrt{P_{0}}\left[\alpha \exp j\left(\omega_{1} t\right)+(1-\alpha) \exp j\left(\omega_{2} t\right)\right] \beta L_{M Z} \times \exp j \theta_{k} \\
& +\sqrt{P_{0}}\left[(1-\alpha) \exp j\left(\omega_{1} t\right)+\alpha \exp j\left(\omega_{2} t\right)\right](1-\beta)
\end{aligned}
$$

Where $\theta_{k}$ is the multilevel phase modulation of the optical carrier.

The two optical carriers are combined asymmetrically to compensate the losses incurred by the I/Q MZM.

The photo-detected signal consists of two components: one baseband, and the other the RF component. The base-band component contributes 
to the DC current, whose value determines the noise of the system. The data RF photo current and the DC photo-current as expressed as follows:

$$
\begin{aligned}
i_{D C} & \approx \frac{1}{2} \mathfrak{R} P_{i n} L_{t o t}\left[\alpha^{2}+(1-\alpha)^{2}\right]\left[\beta^{2}+L_{M Z}^{2}(1-\beta)^{2}\right] \\
i_{R F}(t) & \approx \mathfrak{R} P_{i n} L_{t o t} \sqrt{L_{M Z}} \beta(1-\beta)\left[\alpha^{2}-\left(1-\alpha^{2}\right)\right] \cos \left(\omega_{R F} t+\theta\right)(4.7)
\end{aligned}
$$

Considering the noise generated in the systems, there are two main contributions: intensity noise and the phase noise. The contribution to the intensity noise is from the laser relative intensity noise (RIN), PD shot noise, and thermal noise. Due to the laser's linewidth, the phase of the emitted light signal exhibits a Gaussian random walk. When the two optical signals travel in different paths and have different delays, when combined and heterodyne detected, RF phase noise is generated whose variance can be expressed as:

$$
\sigma_{\phi}^{2}=2 \pi \Delta \nu \tau
$$

Where $\Delta \nu$ is the laser linewidth and $\tau$ is the observation time. The performance limitations of the proposed system are evaluated similar to the previous chapter from the expression of the $\mathrm{DC}$ and $\mathrm{RF}$ currents, where by varying the system parameters, the SNR is calculated. The parameters that are identified to be crucial are $\alpha$ the WDM-DMUX extinction ratio, $\beta$, the coupling ratio, and $L_{M Z}$ the Mach-Zehnder modulator insertion loss. Figure 4.6(a) plots the variation of SNR by the optical power and it is seen that the SNR increases with increased optical power. Similarly, the WDM-DMUX extinction ratio is varied and the SNR plots in fig. 4.6(b) and when the equally distributed both the optical carriers, a complete cancellation of RF signal happens, which is evident from the plot. As mentioned previously asymmetric coupling ratio should be used for coupling the modulated and un-modulated carriers to compensate for the losses of the I/Q MZM. Figure 4.6(c) the coupling ratio of the system $(\beta)$ is varied for different values of Mach-Zehnder insertion losses $\left(L_{M Z}\right)$ and it is observed from fig. 4.6(c) that for optimum performance the coupling ratio $\beta$ has to be chosen in such a way to compensate for the insertion losses of the I/Q MZM. The new value of $\beta$ can be expressed as follows:

$$
\beta \sqrt{L_{M Z}}=1-\beta, \quad \beta=\frac{1}{1+\sqrt{L_{M Z}}}
$$



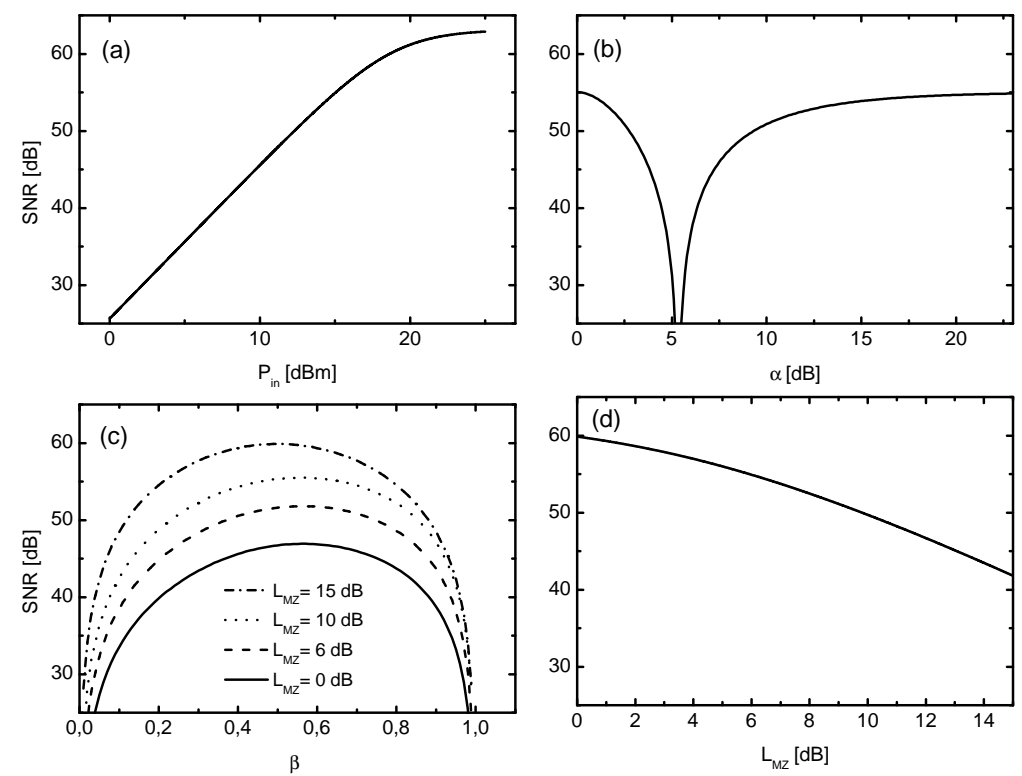

Figure 4.6: Signal to noise ratio of the wireless signals generated using the $\mathrm{mmW}$ modulator. The variation of SNR plotted against the optical power (a), the filter extinction ratio $(\alpha)(\mathrm{b})$, the coupling ratio $(\beta)(\mathrm{c})$, and the I/Q MZM losses (d).

Following the eqn. 4.9 , the MZ insertion losses were varied, and as the modulator loss increases, the SNR decreases with the reduction in total optical power.

\subsubsection{Differential Phase Modulated Wireless Links [Paper J]}

Differential phase modulated wireless signals can be generated using the mmW modulator. Since the I/Q MZM can be used to generate (D)QPSK signals, the generated RF signals can be demodulated using optical technique involving delay interferometric demodulation. The wireless signals that are received at a remote BS can be sent to a CS where optical delay interferometric demodulation can be performed. Figure 4.7 shows the schematic of the RoF uplink. Similar to the PVdM case, the received wireless signals modulate an optical carrier in an external modulator and 
transmitted over a fibre to the CS. Since the RF signals have the same amplitude and phase modulation like an optical DQPSK signal at the transmitter, the amplitude and phase of the original signal is present on both the side-bands. Filtering one of the side-bands the optical DQPSK signal is recovered which is demodulated using DMZI based receiver.

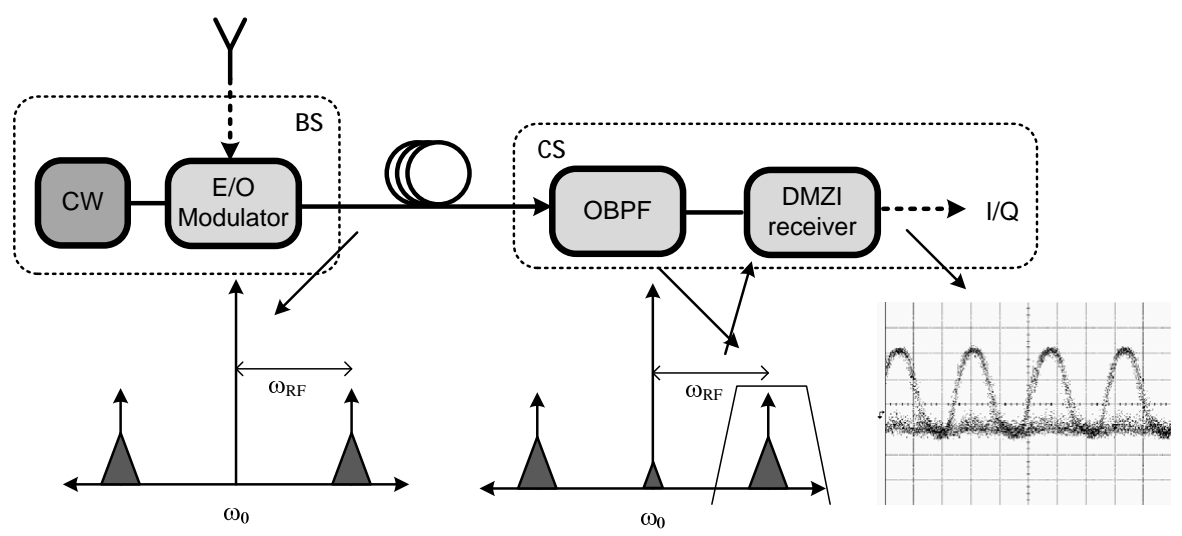

Figure 4.7: Schematic of a radio over fibre uplink with interferometric demodulation for differential phase modulated wireless signals. Inset the demodulated eye diagram of a $60 \mathrm{GHz} 5 \mathrm{~Gb} / \mathrm{s}$ DPSK wireless signal.

In paper $\mathrm{J}$, a proof of concept experimental demonstration of generation of $5 \mathrm{~Gb} / \mathrm{s}$ DPSK signal at $60 \mathrm{GHz}$ carrier is presented. To generate the wireless signals two optical carriers $60 \mathrm{GHz}$ separated were generated using an MZM biased at MiTB. The two optical tones are filtered and one of the carriers is modulated with a $5 \mathrm{~Gb} / \mathrm{s}$ pseudo random bit sequence to generate $5 \mathrm{~Gb} / \mathrm{s}$ NRZ-DPSK optical signal. Using an asymmetric coupler of ratio 10:90, the optical $5 \mathrm{~Gb} / \mathrm{s}$ signal and the unmodulated carrier were combined and photo detected. The output of the photo-detector is a 5 $\mathrm{Gb} / \mathrm{s} 60 \mathrm{GHz}$ DPSK signal. The demodulation of the wireless signals was performed by modulating the $60 \mathrm{GHz} \mathrm{RF}$ carriers on another optical carrier, and filtering one of the side-bands. The filtered side-band with the original DPSK data is fed into a DMZI to demodulate the DPSK signals. To analyse the quality of the signals, prior to demodulation, the optical signals are passed through a pre-amplified receiver and demodulated using a DMZI and photo-detected using a single ended photo-receiver. The quality is quantified by measuring the BER vs. received optical power as 


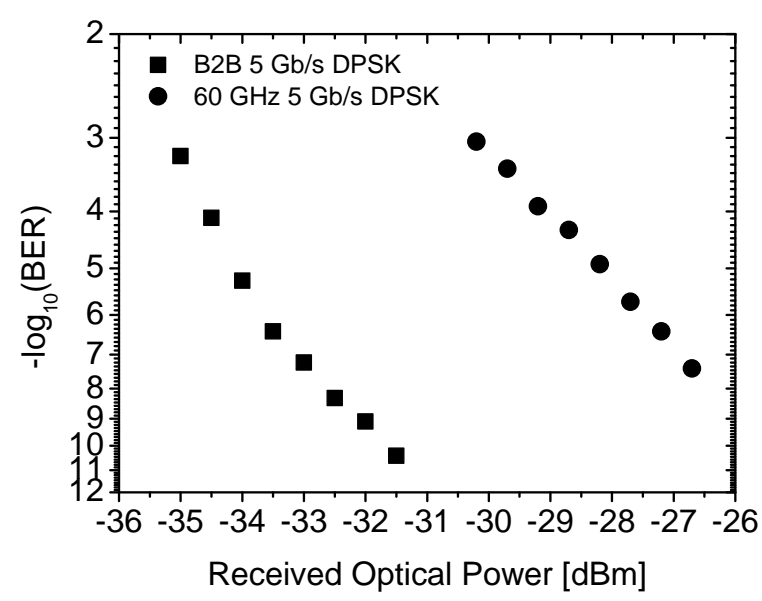

Figure 4.8: Bit error ratio plots of a $5 \mathrm{~Gb} / \mathrm{s}$ optical DPSK signal compared with $5 \mathrm{~Gb} / \mathrm{s} 60 \mathrm{GHz}$ DPSK wireless signals.

plotted in fig. 4.8. From the results it is demonstrated that the wireless DPSK signals can be demodulated with a typical DMZI, and the uplink architecture implemented is immune to fibre's chromatic dispersion induced RF power fading. The results compare an optical DPSK signal in a back-to-back scenario, with an up-converted $60 \mathrm{GHz}$ DPSK signal. For a BER of $10^{-6}$ a power penalty of around $5 \mathrm{~dB}$ is observed which is due to the up-conversion and down-conversion.

\subsection{Optical Digital Coherent Detection}

The constant demand of data capacity has pushed the optical communication systems towards higher spectral efficiency, by using multi level modulation formats, reducing the spacing between the WDM channels, and using polarization multiplexing. The current detection techniques based on direct detection offer good performance but with lower spectral efficiency. Coherent optical detection on the other hand is known for higher sensitivity compared to direct detection based systems, but analogue coherent receivers require phase locked loop with low linewidth lasers [149. In coherent detection the field of the optical signal is detected as the in-phase and quadrature components, which allows greater 


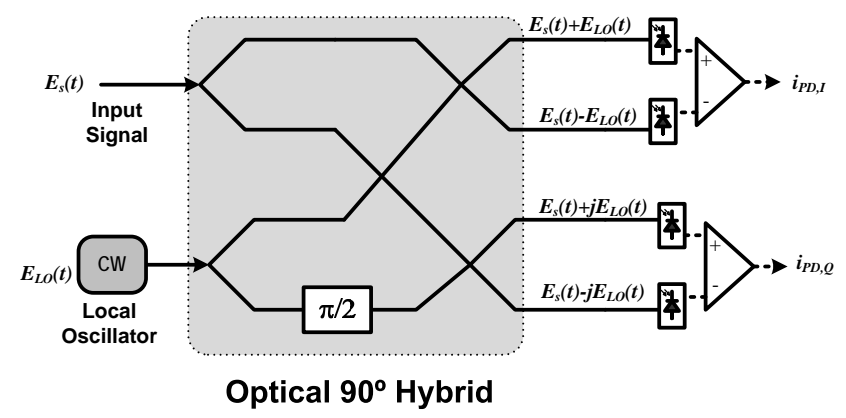

Figure 4.9: Schematic diagram of an optical coherent receiver.

degree of freedom where the phase and polarization multiplexing can be implemented at the transmitter. With the recent developments in electrical technology 150, coherent detection has gained new interest where coherent detection in combination with digital signal processing (DSP) can be used. The advantages of DSP is that no analogue phase locked loops are required, and new adaptive algorithms can be used for detection and for compensating the channel impairments 151 153]. The use of digital techniques over analogue phase locked loops help reduce the cost by allowing integration of coherent receivers with electronics 154, 155.

Figure 4.9 shows the schematic of an optical coherent receiver. In an optical coherent receiver, the optical signal is mixed with a local oscillator using an optical 90 degrees hybrid. A phase shift of $\pi / 2$ is introduced between the upper and lower arms, when balanced photo-detected, the upper arm contains the in-phase component of the optical field and the lower arm the quadrature. The photo-currents of the in-phase and quadrature components can be expressed as:

$$
\begin{aligned}
& i_{P D, I}=2 \Re P_{s} P_{L O} \cos \left(\omega_{s} t-\omega_{L O} t+\phi_{s}+\phi_{L O}\right) \\
& i_{P D, Q}=2 \Re P_{s} P_{L O} \sin \left(\omega_{s} t-\omega_{L O} t+\phi_{s}+\phi_{L O}\right)
\end{aligned}
$$

Where $P_{s}, P_{L O}$ are the signal and LO power. $\omega_{s}, \omega_{L O}$ are the optical wavelengths of the signal and the LO, whereas $\phi_{s}$ and $\phi_{L O}$ are the time varying phase of the optical signal and the LO including the phase noise. One direct advantage of the coherent receiver that can be seen from the eqn. 4.11 is that the balanced detection removes the DC components generated by photo-detection which brings the system to shot noise limit, 


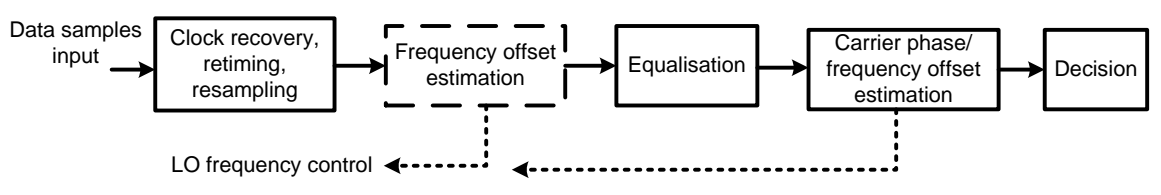

Figure 4.10: Block diagram of a digital signal processing demodulator.

which otherwise would be RIN limited. There is a phase and frequency offset between the signal and the LO, which must be estimated using digital signal processing for successful demodulation of the data.

\subsubsection{Digital Demodulation}

To perform the demodulation of the optical signals using coherent receiver, the photo-detected in-phase and quadrature components are first sampled using an analogue to digital converter (ADC). From the sampled data, the signal is reconstructed in a digital domain, which is expressed as:

$$
e(n)=i_{P D, I}(n)+j i_{P D, Q}(n)
$$

The above equation is normalised and can be expressed as:

$$
e(n)=E_{k}(n) \exp j(\omega t+\phi(n))+p(n)
$$

Where $E_{k}$ is the digital information modulated on the signal. $\omega=\omega_{s}-$ $\omega_{L O}$, is the difference in the wavelengths between the signal and the LO. $\phi(n)=\phi_{s}-\phi_{L O}$ is the phase difference between the signal and LO. The linewidth of the signal and the LO lasers which have a Lorentzian shape exhibit a Gaussian random walk. The phase noise of the lasers is a Weiner process, and the variance of the phase noise is expressed as $\sigma_{\phi}^{2}=2 \pi \tau\left(\Delta \nu_{s}+\Delta \nu_{L O}\right)$. Where $\Delta \nu$ is the linewidth of the laser.

Figure 4.10 shows the block diagram of a DSP demodulator. With the phase and amplitude information of the optical field available in digital domain, digital techniques common to radio communications can be implemented. The first step of the demodulation process is the clock recovery and re-sampling. Re-sampling is performed when the sample rate is not a multiple of the signal baud-rate. The frequency difference between the LO and the signal can be easily estimated by using some known 
techniques common to radio communications [156], or by estimating the phase rotation factor in the DSP 157. A feedback control to control the frequency of the LO is helpful in reducing the frequency drifts, which would improve the performance of the following demodulation blocks.

The next step is the implementation of electronic equalisation which is used to correct the deterministic linear channel impairments. The equalisation can be performed using finite impulse response (FIR) filters 158, 159. Adaptive filtering can be used when the signal is perfectly symmetrical, and for the case of QPSK modulation where all the points are on a circle, least mean squares algorithms such as the common modulus algorithm (CMA) can be used 160,161]. The filters taps with CMA are chosen by minimizing the variance between the symbols and the circle.

The main process of demodulation is the carrier phase estimation. The carrier phase estimation can also estimate the frequency offset of the lasers which is one of the crucial parameters in optical coherent detection. Several algorithms for phase estimate exist [117, 157, 162, 163, and depending on the algorithm, the linewidth tolerance can be from several megahertz to a few gigahertz. It is identified that [157] the power law phase estimate 164 in combination with Wiener filtering has performance similar to a maximum a-priori estimate for phase modulated signals. The rest of the chapter uses the power law phase estimation for demodulation.

In the power law phase estimation, first the phase modulation is removed (in blocks) by raising the M-PSK signal to the Mth power, which for the case of QPSK is 4.

$$
s(n)=\exp j(4 \phi(n))
$$

To estimate the phase, a Wiener filter is used, which can be implemented either using a zero-lag filter where all the $n$ symbols are looked for estimation, or a block of $N$ symbols is used. Both the filters have the advantage of reducing the Gaussian additive noise by averaging the samples, whereas a lower block size is desired in phase noise dominant systems, for better phase tracking. The Wiener filtering can be expressed as:

$$
t(i)=\sum_{i=0}^{N} a_{i} s(i)
$$


where $a_{i}$ are the Wiener filter coefficients. The phase of the filtered samples is divided by M (4) such that the phase values fall between $-\pi / 4$ and $\pi / 4$.

$$
\hat{\phi}=\frac{1}{4} \arg [t(n)]
$$

The estimated phase is now compared with the original signal and then decision is implemented.

\subsubsection{Radio over Fibre Demodulating Downlink [Paper K]}

Till now the use of coherent detection for the demodulation of optical signals was addressed, and the common DSP algorithms used for demodulation of phase modulated optical signals described. Coherent detection can be advantageous for RoF signal detection because of the higher sensitivity demonstrated by coherent detection. The use of coherent detection will allow use of digital demodulation and equalisation techniques common to radio communications using DSP, and all the impairments caused by the wireless and optical channel can be correct without the need of $\mathrm{mmW}$ components. With the recent advancements in coherent detection it is evident that in future coherent detectors will form integral part of network equipment, and using coherent detection for both optical baseband and RoF signals will reduce the cost. Coherent detection will play a crucial role for demodulating very high capacity wireless links, since currently there is no mature technology which can provide the same performance.

Currently the demodulation techniques for RoF links involve the downconversion of the RF signal directly to baseband or IF frequency which would require RF components installed either in the BS or the CS. In paper $\mathrm{K}$ a novel RoF uplink scheme with coherent detection is described, which is illustrated in fig. 4.11. Similar to the photonic vector demodulation as described in the previous section, the received wireless signals at the BS modulate an optical carrier generated in an external electro-optic modulator in simple DSB modulation, and fed into the SMF. Considering a wireless signal according to the expression in eqn. A.1 of Appendix 1 , the expression of the optical signal when modulated by the vector 


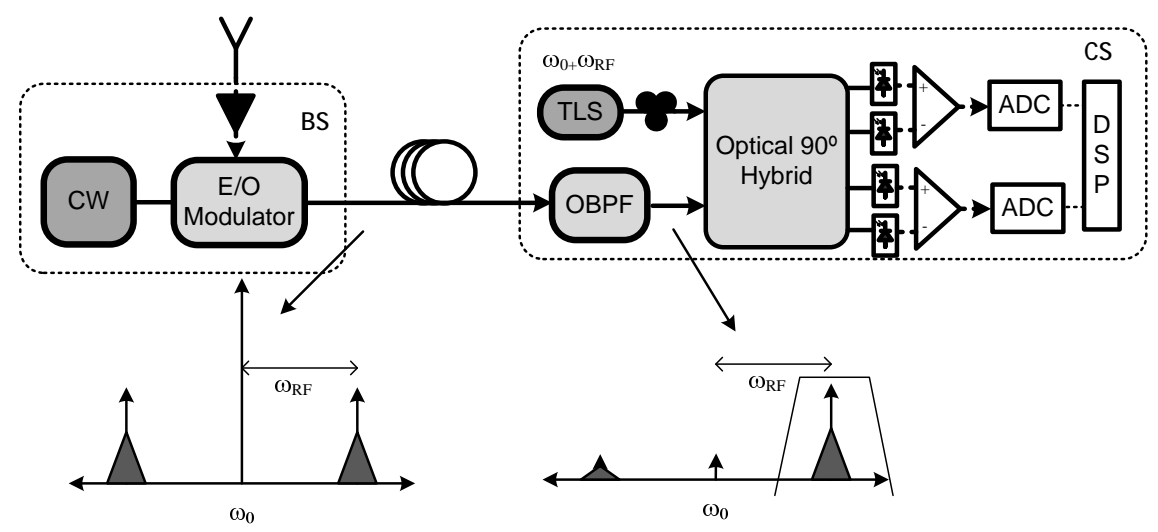

Figure 4.11: Illustration of a radio over fibre downlink with coherent detection based demodulation. TLS- tunable laser source.

modulated wireless can be expressed as:

$$
\begin{aligned}
E_{\text {out }}(t)= & \sqrt{P_{0} T_{f f}} \exp j\left(\omega_{0} t+\phi_{0}\right)\left[J_{0}\left(m_{R F}\right)+J_{1}\left(m_{R F}\right)\right. \\
& \left.A(t) \cdot \exp j\left( \pm \omega_{R F} t+\phi_{R F}+\Psi(t)+\frac{\beta_{2} L \omega_{R F}^{2}}{2}\right)\right]
\end{aligned}
$$

Where $\omega_{0}$ and $\phi_{0}$ are the frequency and phase of the optical carrier. $\phi_{R F}$ is the phase of the RF wireless signal. The RoF signals with the $\mathrm{RF}$ modulation on both the side-bands is mixed with another optical LO signal in an optical $90^{\circ}$ hybrid. Homo-dyne coherent detection is performed on one of the side-bands which contain the RF modulation by tuning the LO laser to the optical frequency of the side-band $\left(\omega_{0}+\omega_{R F}\right)$. The balanced photo-detected in-phase and quadrature components can be expressed as:

$$
\begin{aligned}
& i_{R F}^{I}(t)=2 \Re \sqrt{2 P_{0} P_{s} L_{t o t}} A(t) \cos \left(\Delta \omega t+\phi_{s}+\Psi(t)+\frac{\beta_{2} L \omega_{R F}^{2}}{2}-\phi_{L O}\right) \\
& i_{R F}^{Q}(t)=2 \Re \sqrt{2 P_{0} P_{s} L_{t o t}} A(t) \sin \left(\Delta \omega t+\phi_{s}+\Psi(t)+\frac{\beta_{2} L \omega_{R F}^{2}}{2}-\phi_{L O}\right)
\end{aligned}
$$

Where $\Delta \omega=\omega_{0}+\omega_{R F}-\omega_{L O}$ is the frequency deviation between the sideband and the LO signal, and $\phi_{s}=\phi_{0}+\phi_{R F}$ is the total phase of the side-band. Using DSP, the phase and frequency offset are estimated and the in-phase and quadrature components are detected. 
The coherent detection based RoF signal demodulation is immune to the chromatic dispersion induced RF power fading since the detection is performed only on one of the side-band. The in-phase and the quadrature components at the output of the balanced photo-receivers are sampled using an ADC with a sampling rate at-least twice the baud-rate and digital demodulated. The added advantage of this proposed technique is that it is transparent to the RF frequency where the same receiver can be used for any RF frequency simply by changing the frequency of the LO laser. In paper $\mathrm{K}$ demodulation of a $2.5 \mathrm{~Gb} / \mathrm{s} 40 \mathrm{GHz}$ QPSK

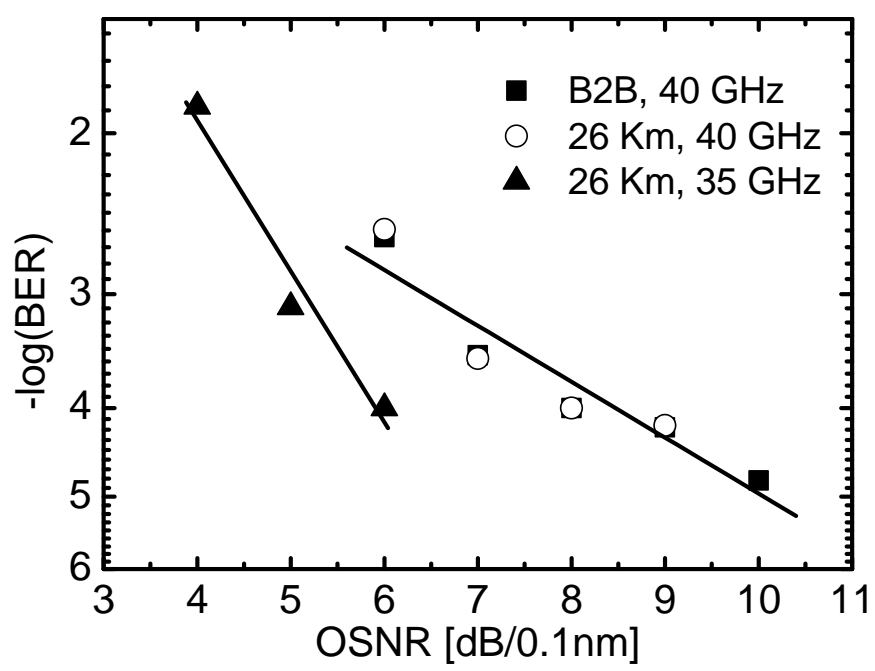

Figure 4.12: Bit error ratio plots of the demodulated $2.5 \mathrm{~Gb} / \mathrm{s}$ QPSK signal at $40 \mathrm{GHz}$ and $35 \mathrm{GHz}$ with $26 \mathrm{~km}$ of SMF transmission.

signal was performed using the above mentioned technique. First the wireless signals were demodulated in a back-to-back scenario where no fibre was placed between the BS and the CS. To measure the quality of the demodulated signals BER was calculated from the measured data by varying the OSNR. BER values of below $10^{-4}$ were recorded with OSNR values as low as $8 \mathrm{~dB}$ as shown in fig. 4.12 , demonstrating the sensitivity of the demodulation technique. Later, a $26 \mathrm{~km}$ of SMF was placed between the BS and the CS, and from the BER measurements no additional OSNR penalty was observed, proving the techniques immunity to chromatic dispersion induced RF power fading. The same experiment was performed at $35 \mathrm{GHz}$, and the results show that the RoF uplink architecture is transparent to the RF frequency. 


\subsection{Optical Heterodyne Generation of Millime- tre Wireless Signals [Paper L]}

In the previous section the use of coherent detection for both baseband and wireless signal demodulation was discussed. The coherent detection in combination with DSP enables the use of free running LO lasers for coherent detection without the need of analogue optical phase locked loops. The various radio communication techniques for digital demodulation, filtering, and equalisation techniques can be implemented using DSP to correct the phase and frequency offsets in homo-dyne coherent detection.

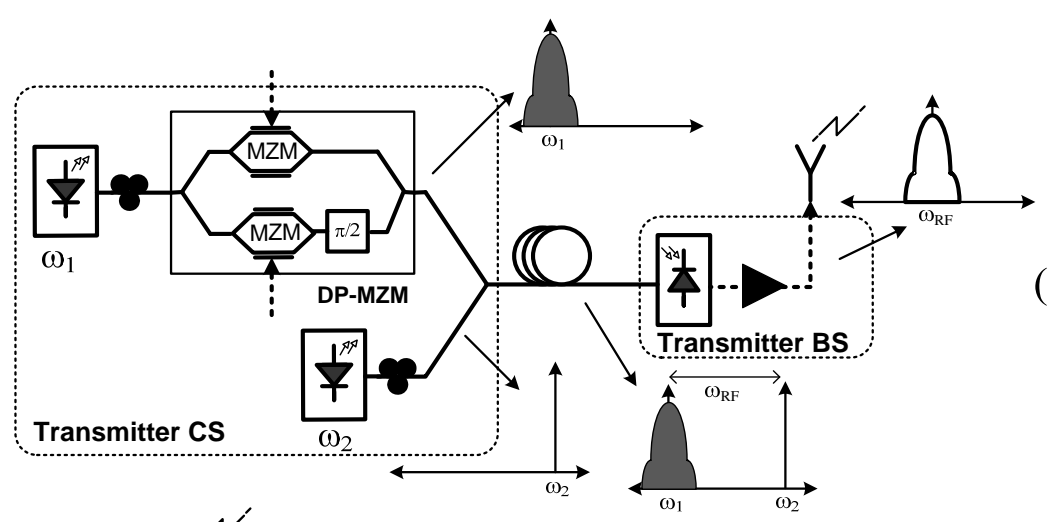

(a)

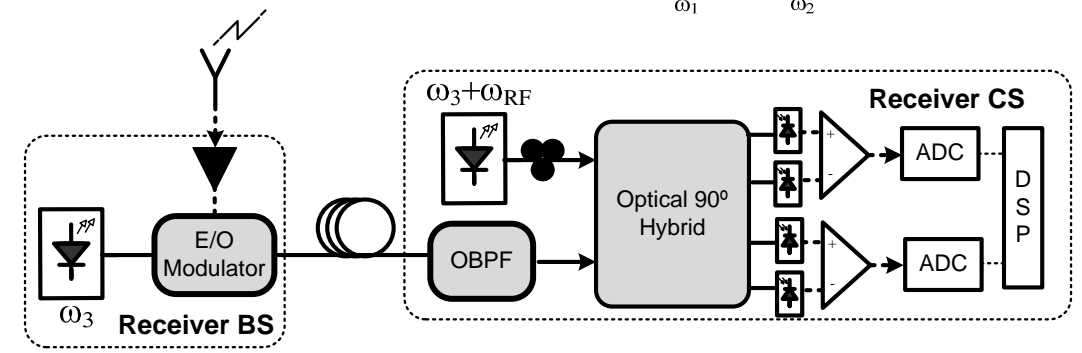

(b)

Figure 4.13: Illustration of a wireless signal generation set-up using optical heterodyne mixing (a), and the radio over fibre uplink with an optical coherent receiver based demodulator (b).

In the previous sections a modulator concept for use generating $\mathrm{mmW}$ wireless signals is presented. The proposed modulator can be used to 


\subsection{Optical Heterodyne Generation of Millimetre Wireless Signals}

[Paper L]

generate different modulation formats since it incorporates an I/Q MZM, but would require integrated devices if modulation formats like QAM have to be implemented.

On the same principles of coherent detection, optical heterodyne detection for generation of wireless signals was demonstrated involving optical phase locked loop techniques 76, 77. Optical phase locked loop based wireless signal generation have similar drawbacks related to analogue optical phase locked loops like low linewidth requirement, strict group delays. Apart from the aforementioned problems, for generating higher frequencies in the $\mathrm{mmW}$ band, complex electronic circuitry is needed which limits the use of optical phase locked loops for $\mathrm{mmW}$ wireless.

Digital signal processing aided optical coherent detection has allowed the use of free running lasers for homo-dyne mixing and correcting the resulting phase and frequency offsets. Using the advantages of optical coherent detection and DSP, in paper L a new technique for generation of wireless signals by heterodyne mixing of two free running optical carriers is presented. In this technique, one of the optical carriers is modulated either in phase, amplitude or a combination of both, while the other unmodulated optical carrier is separated in frequency by the desired RF carrier frequency, which are combined and mixed in a photo-detector. The whole generation process of the wireless signals only require baseband electronics and optical modulator at the baud-rate. The heterodyne mixing of two free running lasers results in phase and frequency offset. To correct these phase and frequency offset, the optical RoF demodulation technique using optical coherent detection, as shown in fig. 4.11 can be implemented.

One of the main advantages of this system is the simplicity, where no $\mathrm{mmW}$ components are needed. Direct optical base-band data can be easily converted to wireless at any desired frequency, which does not require any additional optical/electrical conversion nor RoF equipment. It can be said that the classical RoF problem has been transformed completely into a baseband digital problem, but still for generation, transmission and demodulation of wireless signals. The use of common modulation format for both optical and wireless links provide a complete transparent integration between the wired and wireless networks. 
Figure 4.13 shows the schematic of the heterodyne optical mixing based wireless signal generation and sub-sequent demodulation. The use of optical I/Q MZM and heterodyne mixing for RF signal generation makes this technique scalable in frequency, bit-rate and modulation format. Since only one of the optical carriers is modulated, the resulting signal is SSB in nature, which is immune to the fibre's chromatic dispersion. The downside of this technique is the use of four free-running lasers, which greatly increases the total phase noise of the system which is the sum of the phase noise all the four lasers. Also the frequency and phase offset doubles which will require greater signal processing complexity.

\subsubsection{Experimental Validation}

The increase in capacity demands is pushing the wireless systems towards higher $\mathrm{mmW}$ bands like the $70-80 \mathrm{GHz}$ (W-band). Using the heterodyne mixing technique, theoretically wireless signals at any frequency can be generated as long as the bandwidth of the $\mathrm{PD}$ and the electro-optic modulator at the receiver is sufficient enough. In paper L, experimental demonstration of a wireless signal generation using optical heterodyne mixing and subsequent demodulation using an optical coherent detection is presented. Record capacities of up to $20 \mathrm{~Gb} / \mathrm{s}$ signal generation in the $75-110 \mathrm{GHz}$ band with QPSK modulation is demonstrated. Figure 4.14(a) shows the combined optical spectrum of a $20 \mathrm{~Gb} / \mathrm{s}$ QPSK signal and an un-modulated optical carrier $0.72 \mathrm{~nm}(90 \mathrm{GHz})$ apart . To perform the demodulation the RoF optical coherent detection based demodulation was used, and the BER was measured as a function of received optical power. Using this technique first a $5 \mathrm{~Gb} / \mathrm{s}$ ASK signal was generated at $82 \mathrm{GHz}$ and later at $100 \mathrm{GHz}$. Later the modulation format was changed to QPSK and up to $20 \mathrm{~Gb} / \mathrm{s}$ wireless signals at $82 \mathrm{GHz}$ were generated. Figure 4.14(b) shows the BER plots, indicating very high receiver sensitivity with BER of $10^{-3}$ with received optical power of $-48 \mathrm{dBm}$. In fig. 4.14(b) it can be seen that the use of adaptive equalisation improves the BER for $16 \mathrm{~Gb} / \mathrm{s}$ QPSK signal from $10^{-3}$ to $10^{-5}$ indicating a strong inter-symbol-interference at $16 \mathrm{~Gb} / \mathrm{s}$. This is mainly attributed to the bandwidth of the various electronic and opto-electronic components used. The advantage of using coherent detection is that the device induced imperfections like distortion can be corrected using the 


\subsection{Optical Heterodyne Generation of Millimetre Wireless Signals}

[Paper L]

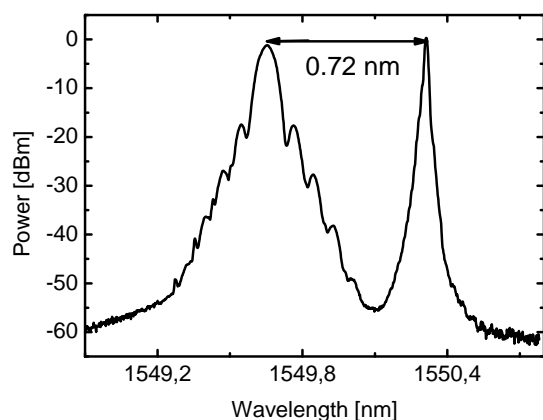

(a)

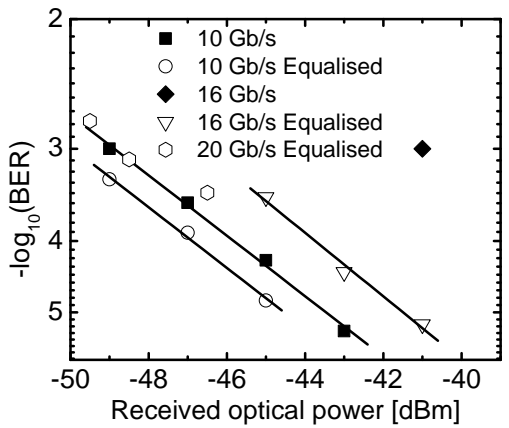

(b)

Figure 4.14: (a)Measured optical spectra of a $20 \mathrm{~Gb} / \mathrm{s}$ QPSK signal together with an unmodulated carrier $90 \mathrm{GHz}$ away. (b) Bit error rate plots of the 75-110 $\mathrm{GHz}$ QPSK wireless signals with up to $20 \mathrm{~Gb} / \mathrm{s}$ capacity.

appropriate equalisation algorithm.

\subsubsection{Optical OFDM based $40 \mathrm{~Gb} / \mathrm{s}$ Wireless Signal Gen- eration [Paper $M$ ]}

Heterodyne mixing of optical signals for generation of wireless signals provide a very interesting solution for high capacity wireless links. Using modulation formats like QPSK upto $20 \mathrm{~Gb} / \mathrm{s}$ wireless signal generation is demonstrated with relatively lower spectral efficiency of around $1 \mathrm{~b} / \mathrm{s} / \mathrm{Hz}$. Advanced modulation formats like 16-QAM can help increase the spectral efficiency but require low phase noise and complex equalisation algorithms which will limit its use. The heterodyne technique involves beating of four free running lasers, which have twice the phase noise compared to an optical baseband link. Recently the use of closely spaced WDM signals for very high capacity baseband optical transmission have been gaining interest 13, 165], which use the advantage of coherent detection and reduce the channel spacing between WDM channels. A novel technique where two or more synchronous optical carriers with a separation of the channel baud-rate in an orthogonal frequency division multiplexing (OFDM) condition to double the channel bit-rate was proposed [165]. Several other results followed, reporting increased spectral efficiency us- 


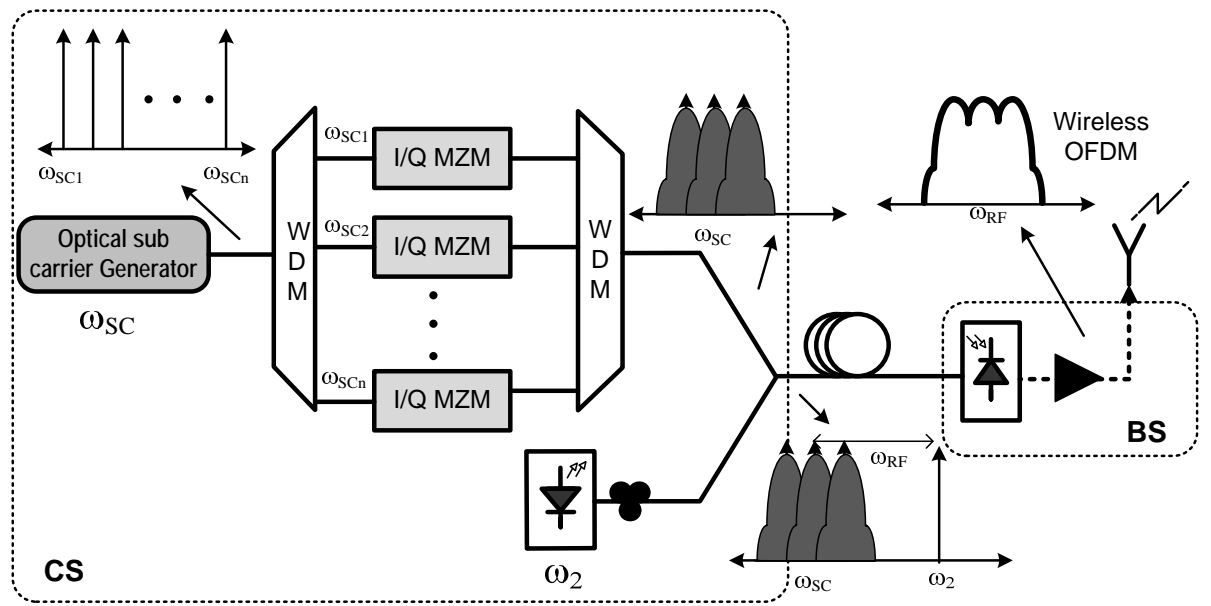

Figure 4.15: Schematic of a wireless OFDM signal generator using heterodyne mixing of an optical OFDM signal.

ing optical OFDM (O-OFDM) modulation. Using this technique upto five sub-carrier QPSK signal generation with a record capacity of 250 $\mathrm{Gb} / \mathrm{s}$ on a single WDM channel was experimentally demonstrated [13].

OFDM signal modulation is widely used in current wireless networks. The current trend in very high capacity $(>10 \mathrm{~Gb} / \mathrm{s}$ ) wireless links is the use of electrical OFDM signals at a lower IF frequency and up-converting to the RF frequency using optical up-conversion techniques seen in chapter 2. These techniques are limited in bandwidth since higher bandwidth require the digital to analogue converters at higher speeds. Also direct integration between optical and wireless signal is not achieved without making the necessary changes in the signal format. In paper $M$, generation of wireless OFDM signals by simply converting an optical OFDM signal to electrical is presented. The generation process is based on heterodyne mixing of an optical OFDM-QPSK signal with an unmodulated carrier. Different sub-carrier configurations were implemented at $60 \mathrm{GHz}$ and $75-110 \mathrm{GHz}$ and up to $40 \mathrm{~Gb} / \mathrm{s}$ wireless signal generation using two sub-carrier QPSK modulation is demonstrated. Figure 4.15 shows the schematic of the wireless OFDM signal generator. Using this technique, first wireless signals with two sub-carriers at a record capacity of $20 \mathrm{~Gb} / \mathrm{s}$ (5 Gbaud) were generated at $60 \mathrm{GHz}$. Later, the carrier frequency was set to be in the $75-110 \mathrm{GHz}$ band and two sub-carrier OFDM signals at 5 


\subsection{Optical Heterodyne Generation of Millimetre Wireless Signals}

[Paper L]

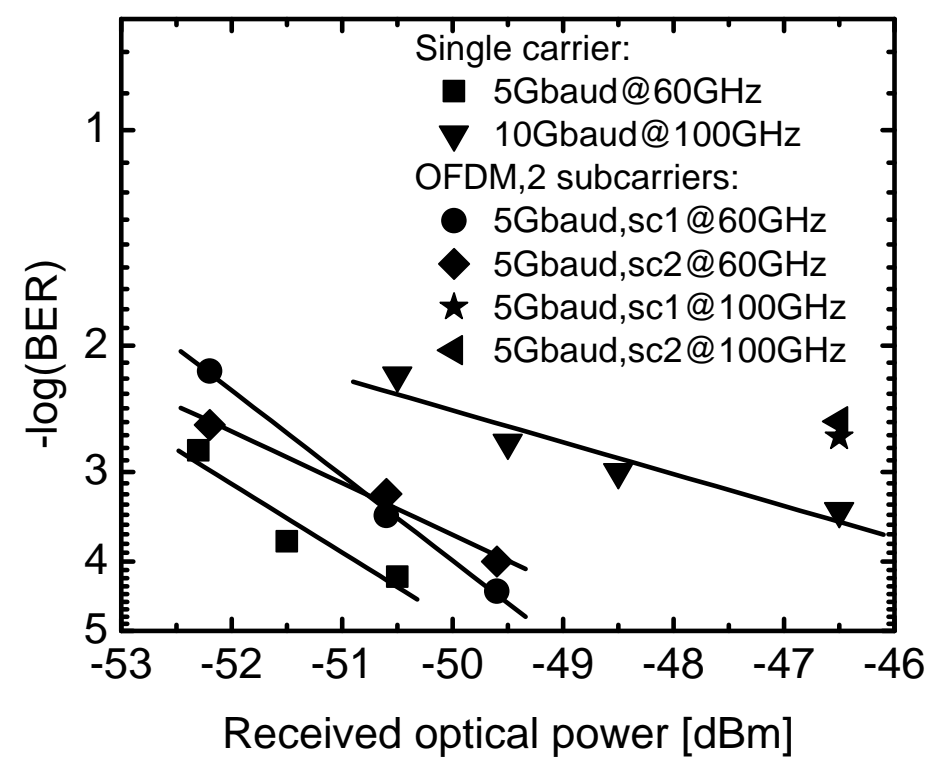

Figure 4.16: Bit error ratio plots of wireless OFDM signals with different sub carriers at $60 \mathrm{GHz}$ and $100 \mathrm{GHz}$ band.

Table 4.1: Summary of different sub carriers for OFDM-QPSK wireless signal generation and their corresponding bit error ratios in both $60 \mathrm{GHz}$ and $75-110$ $\mathrm{GHz}$ bands.

\begin{tabular}{llllll}
\hline \hline $\begin{array}{l}\text { Sub } \\
\text { riers }\end{array}$ & $\begin{array}{l}\text { car- } \\
\text { rate } \\
(\text { Gbaud })\end{array}$ & $\begin{array}{l}\text { Frequency } \\
\text { Band } \\
(\mathrm{GHz})\end{array}$ & $\begin{array}{l}\text { Total } \\
\text { Bit-rate } \\
(\mathrm{Gb} / \mathrm{s})\end{array}$ & $\begin{array}{l}\text { Spectral } \\
\text { efficiency } \\
(\mathrm{b} / \mathrm{s} / \mathrm{Hz})\end{array}$ & $\begin{array}{l}\text { Measured } \\
\text { BER }\end{array}$ \\
\hline 1 & 5 & 60 & 10 & 1.33 & $6.2 \times 10^{-5}$ \\
2 & 5 & 60 & 20 & 1.67 & $3.3 \times 10^{-4}$ \\
3 & 4 & 60 & 24 & 1.71 & $1.7 \times 10^{-3}$ \\
2 & 8 & $75-110$ & 32 & 1.67 & $7.9 \times 10^{-3}$ \\
2 & 10 & $75-110$ & 40 & 1.67 & $8.9 \times 10^{-3}$ \\
\hline
\end{tabular}

Gbaud resulting a total capacity of $20 \mathrm{~Gb} / \mathrm{s}$ were generated . Figure 4.16 shows the BER plots of the wireless OFDM signals.

The relatively large penalty induced by the wireless signals in the $75-110$ $\mathrm{GHz}$ band is due to the severe bandwidth limitations imposed by various components. For example, the amplifier used at $75-110 \mathrm{GHz}$ band has 6 
$\mathrm{dB}$ lower gain compared to the one at $60 \mathrm{GHz}$, and the photo-diode used for wireless signal generation has a $3 \mathrm{~dB}$ cut-off bandwidth of around $90 \mathrm{GHz}$. using the similar technique different sub-carrier configurations at with different baud rates were implemented for generation of OFDM wireless signals and summarised in table

\subsection{Conclusions}

In this chapter the optical digital communication techniques for generation and demodulation of wireless signals is presented. Unlike previous photonic vector modulation techniques, the optical baseband techniques provide the advantage of generating any vector modulated signals by using a simple dual parallel Mach-Zehnder modulator with are tolerant to dispersion induced RF power fading. Optical modulation formats like DQPSK were studied where an optical DQPSK signal is directly converted to an RF DQPSK signal using a special modulator configuration. Similarly a novel concept for generation of wireless signals using heterodyne technique is presented. The heterodyne technique uses two free running lasers to generate the $\mathrm{mmW}$ wireless signals with theoretically any modulation format with any RF frequency. The major advantage of these above techniques is the generation of phase modulated SSB signals, which are immune to chromatic dispersion.

Optical differential phase modulated wireless signal generation was successfully demonstrated and the around $5 \mathrm{~dB}$ of power penalty was observed after the process of up-converting an optical DPSK signal to wireless and later demodulation. The generation technique simultaneously provides wired and wireless signals which could be advantageous in several network architectures.

The optical demodulation techniques are all based on side-band filtering on the same grounds of the photonic vector demodulator. Here, the sideband filtering recovers the original modulation format of the wireless signals on the side-band. The filtered side-band which is a baseband optical signal is demodulated using various incoherent techniques. The optical differential phase modulation based wireless signals were demodulated us- 
ing delay interferometric demodulation, whereas coherent detection was implemented for advanced modulation formats like OFDM.

Optical coherent detection based demodulation has demonstrated very high sensitivities, and the digital signal processing can be used to correct the components induced degradation. The demodulation of a $2.5 \mathrm{~Gb} / \mathrm{s}$ wireless signal was performed after $25 \mathrm{~km}$ of fibre, and no penalty was observed. Also a BER of around $10^{-3}$ was measured with OSNR values as low as $6 \mathrm{~dB}$, demonstrating the high sensitivity of the coherent detection.

The wireless signal generation by simply heterodyne mixing of two free running lasers was investigated, and the DSP aided coherent detection was implemented to track the phase and frequency offsets generated by the free running lasers. Using this technique up to $20 \mathrm{~Gb} / \mathrm{s}$ QPSK wireless signal generation and successful demodulation was performed where receiver sensitivity of around $-48 \mathrm{dBm}$ was recorded. The RF devices induced bandwidth limitations were also corrected using adaptive equalisation techniques indicating the advantages of DSP based coherent detection. The scalability and the frequency transparency of the proposed system was demonstrated by generating wireless signals with different sub carrier OFDM configurations in both 60 and $75-110 \mathrm{GHz}$ bands. Using the optical OFDM techniques up to $40 \mathrm{~Gb} / \mathrm{s}$ wireless signal generation was demonstrated with a two sub carrier OFDM-QPSK modulation.

The major drawback of the heterodyne system is the total phase noise generated by beating of four free running lasers, which will limit the choice of the modulation format. Hence higher spectral efficiency may only be achieved by using higher number of sub carriers while using robust modulation format like QPSK. Similarly the phase and frequency offsets become larger, which to a certain extent can be corrected, but with complex tracking algorithms.

It can be concluded that the direct conversion of optical baseband signals to $\mathrm{RF}$ has the clear advantage of seamless wireless/wired integration, since all the data has to be carried to from the antenna using optical fibre. The possibility of directly feeding the wireless BS with optical baseband data reduces the complexity and the cost of the network to a great extent. Also current optical networks and systems can be readily used to provide 
wireless services. without the need of any radio over fibre techniques. The modulation and demodulation techniques proposed in this chapter are totally transparent and the addition of the wireless channel does not require any change in modulation format maintaining the higher network layers intact. 
Chapter 5

\section{Conclusions and Future Outlook}

Gigabit capacity wireless links are evolving at a very rapid pace which is already estimated to be a multi-billion dollar market, and wireless links matching capacities of optical communication links are extensively investigated. The main reason to seek such high capacity wireless is to bridge the existing bottle neck between the wired and wireless communication systems, and providing such high capacities to users will open new opportunities and markets. To generate and support the high capacity wireless, optical communications as a backbone plays a very crucial role. Radio over fibre technology offers the flexible combination of wireless and optical communications for generation, transmission and demodulation of wireless signals, bring optics and wireless closer.

The current state of the art in wireless technology is moving towards achieving very high capacities in wireless, mostly using high spectral efficient electrical signals and later up-conversion to RF frequency using optical techniques. This technique offers a good solution for providing wireless connectivity but has the several limitations like fibre's dispersion, limited bandwidth scalability, and complex electronic modulators and 
demodulators at intermediate frequency. The current radio over fibre uplinks have a similar limitation, which either transport the whole RF signal to the central station which is dispersion prone, or use an IF downconversion which would require IF demodulators.

In this work, several techniques for generation and demodulation of wireless signals are presented. The main difference and advantage between this work and the previously existing work in the use of pure baseband signalling for generating the wireless signals with advanced modulation formats. The use of pure baseband signals does not require any RF or IF components, and direct optical baseband signals can be used to generate wireless signals, which provides a greater integration between wireless and optical signals. Similarly the demodulation techniques proposed convert the wireless signals to optical baseband signals which are immune to chromatic dispersion.

\section{Photonic Vector Modulation/Demodulation}

Photonic vector modulation provides a simple solution to convert optical on-off-keying signals to wireless QAM signals. The results indicate very good quality when PVM technique is used to generate the wireless signals. The PVM can be implemented either at the CS or baseband data can be directly fed to an antenna BS. Higher spectral efficient modulation formats can be generated using PVM, and generation of $10 \mathrm{~Gb} / \mathrm{s}$ signals at $60 \mathrm{GHz}$ using QPSK and 16-QAM modulation were demonstrated. The robustness of PVM based wireless signals was demonstrated by feeding it into a reconfigurable optical access network with different other services in a DWDM scenario.

The main drawback of these systems is the fibre's chromatic dispersion, which will limit the transmission distance as higher mmW bands are considered. This is due to the use of conventional optical RF up-conversion techniques using external modulators in a double side-band configuration.

Radio over fibre uplinks have always been limited by the fibre's chromatic dispersion. Using the properties of optical side-band filtering of 
a radio over fibre signal, photonic vector demodulation offers the ability to directly demodulate wireless signals to their respective in-phase and quadrature component in baseband. The direct conversion of RF signal to baseband optical signal offers immunity to fibre's chromatic induced dispersion. Also the direct conversion of RF to baseband will allow the possibility of feeding the baseband signals directly into a higher optical network like metro or core.

The only limitation of the demodulating downlink is the requirement of phase locked electrical LO signal at the central station, which will complicate at higher $\mathrm{mmW}$ frequencies.

\section{Optical Coherent Detection}

The renewed interest in optical coherent detection is obvious in the field of optical communications. The major advantages of using optical coherent detection is the high sensitivity obtained, and combining coherent detection with digital signal processing creates robust optical communication systems where several device and transmission impairments can be corrected using digital signal processing. In this work the use of optical coherent detection in combination with DSP for the detection of wireless signals is proposed. In a downlink scenario, the side-band filtering offers the dispersion immunity and the coherent detection the required sensitivity. Since coherent receivers will be available in every network equipment in the near future, use of coherent detection is a very attractive solution for radio over fibre links which will reduce the cost drastically, and achieve seamless integration between the wireless and wired signals.

\section{Optical Heterodyne Based Wireless Signal Gen- eration}

The robustness of optical coherent detection and digital signal processing, which enables the use of free running lasers for homo-dyne detection has motivated the use of optical heterodyne mixing for wireless signal 
generation. In this technique a wireless signal is simply generated by heterodyne mixing with another free running laser, virtually possible to generate any modulation format at any carrier frequency. Wireless signal generation at capacities equivalent to optical systems have been demonstrated, with up to $40 \mathrm{~Gb} / \mathrm{s}$ in the $75-110 \mathrm{GHz}$ band. This technique converts a radio over fibre system to a pure baseband system, but still providing wireless services. The advantages of this technique is that no $\mathrm{RF}$ components are required either for generation nor for demodulation, and any optical WDM node can be easily converted into a wireless base station. Also this technique is agnostic to the added wireless channel, which achieved a seamless transparent integration.

The downside of this technique is the total phase noise generated in the system due to the mixing of four free running lasers. This would limit the modulation format that can be used, creating an upper limit to the spectral efficiency. On the other hand optical OFDM techniques can be used to increase the spectral efficiency while maintaining a lower order robust modulation format like QPSK.

\section{Outlook}

Broadband wireless links integrated with optical access networks need to mature before becoming a reality. The integration of wireless and wired services will be the crucial motivation for implementation of such network architectures. Photonic generation techniques offer the flexibility, but transparency at higher network protocol layers must be considered.

The optical downlinks carrying these high capacity wireless link mainly rely on the electro-optic modulator which operate at the $\mathrm{mmW}$ frequency. Currently modulator up to $100 \mathrm{GHz}$ are available, but when higher frequency bands are considered, devices working at such high frequencies must be investigated.

Optical coherent detection is maturing and commercial implementation of such devices will happen in the near future. Coherent detection in radio over fibre will play a crucial link, especially demonstrating the ability of 
direct conversion of optical baseband to RF signal. new algorithms can be investigated which will provide more robustness to phase noise, and higher linewidth devices like VCSELS can be used to reduce the cost of the systems. 
Appendix $\mathrm{A}$

\section{Expressions of Photonic Vector Demodulator}

Assuming the wireless QAM signals to be of the form:

$$
\begin{aligned}
V_{R F}(t) & =V_{m}\left[I(t) \cos \left(\omega_{R F} t+\phi_{R F}\right)+Q(t) \sin \left(\omega_{R F} t+\phi_{R F}\right)\right] \\
& =V_{m} A(t) \sin \left(\omega_{R F} t+\phi_{R F}+\Psi\right)
\end{aligned}
$$

Where $A(t)=\sqrt{I^{2}(t)+Q^{2}(t)}$ and $\Psi=\arctan \left(\frac{Q(t)}{I(t)}\right)$

Using a MZM, to modulate the wireless signal on an optical carrier, the output of the MZM after L km of SMF can be written as:

$$
\begin{aligned}
E_{\text {out }}(t)= & \sqrt{P T_{f f}} \exp j\left(\omega_{0} t+\phi_{0}\right) \cdot\left[J_{0}\left(m_{R F}\right)+J_{1}\left(m_{R F}\right)\right. \\
& \left.A(t) \cdot \exp j\left( \pm \omega_{R F} t+\phi_{R F}+\Psi+\frac{\beta_{2} L \omega_{R F}^{2}}{2}\right)\right]
\end{aligned}
$$

Modulating the above with a LO tone of frequency $\omega_{L O}=\omega_{R F}$ :

$$
\begin{aligned}
E_{\text {out }}(t)= & \sqrt{P} T_{f f} \exp j\left(\omega_{0} t+\phi_{0}\right) \\
& \cdot\left[J_{0}\left(m_{R F}\right)+J_{1}\left(m_{R F}\left(A(t) \exp j\left( \pm \omega_{R F} t \pm \phi_{R F}+\Psi+\frac{\beta_{2} L \omega_{R F}^{2}}{2}\right)\right]\right.\right. \\
& \cdot\left[J_{0}\left(m_{L O}\right)+J_{1}\left(m_{L O}\right) \exp j\left( \pm \omega_{R F} t \pm \phi_{L O^{+}}\right]\right.
\end{aligned}
$$


Simplifying the above expression and deriving only up to the first order term:

$$
\begin{aligned}
E_{\text {out }}(t)= & \sqrt{P} T_{f f} \exp j\left(\omega_{0} t+\phi_{0}\right) \cdot\left[J_{0}\left(m_{R F}\right) J_{0}\left(m_{L O}\right)+\right. \\
& \cdot J_{0}\left(m_{R F}\right) J_{1}\left(m_{L O}\right) \exp j\left( \pm \omega_{R F} t \pm \phi_{L O}\right)+J_{0}\left(m_{L O}\right) J_{1}\left(m_{R F}\right) \\
& \left.A(t) \exp j\left( \pm \omega_{R F} t \pm \phi_{R F}+\Psi+\frac{\beta_{2} L \omega_{R F}^{2}}{2}\right)\right]
\end{aligned}
$$

Now fltering the upper sideband at $\omega_{0}+\omega_{R F}$, we get:

$$
\begin{aligned}
E_{\text {out }}^{U S B}(t)= & \sqrt{P} T_{f f} \exp j\left(\omega_{0} t+\omega_{R F} t+\phi_{0}\right)\left[J_{0}\left(m_{R F}\right) J_{1}\left(m_{L O}\right) \exp j\left(\phi_{L O}\right)\right. \\
& \left.+J_{0}\left(m_{L O} J_{1}\left(m_{R F}\right) A(t) \exp j\left(\phi_{R F}+\Psi+\frac{\beta_{2} L \omega_{R F}^{2}}{2}\right)\right)\right] \quad \text { (A.5) }
\end{aligned}
$$

For the in-phase component the phase relation between the LO and RF signals is:

$$
\phi_{L O}=\phi_{R F}+\frac{\beta_{2} L \omega_{R F}^{2}}{2}
$$

The photo-detected output of the in-phase component is:

$$
i_{P D}^{I}(t)=\frac{P T_{f f}^{2}}{2} J_{0}^{2}\left(m_{R F}\right) J_{1}^{2}\left(m_{L O}\right) I(t)
$$

For quadrature component the phase relation between the LO and RF signals is:

$$
\phi_{L O}=\phi_{R F}+\frac{\beta_{2} L \omega_{R F}^{2}}{2}+\frac{\pi}{2}
$$

Thus, the photo-detected output of the quadrature component is

$$
i_{P D}^{Q}(t)=\frac{P T_{f f}^{2}}{2} J_{0}^{2}\left(m_{R F}\right) J_{1}^{2}\left(m_{L O}\right) Q(t)
$$




\section{Bibliography}

[1] A. M. Odlyzko, "Internet traffic growth: sources and implications," in Optical Transmission Systems and Equipment for WDM Networking II, (Orlando, FL), 2003.

[2] R. J. Mears, L. Reekie, I. M. Jauncey, and D. N. Payne, "Low-noise Erbium doped fibre amplifier operating at $1.45 \mu \mathrm{m}$," Electron. Lett., vol. 23, no. 19, pp. 109-111, 1987.

[3] P. R. Trischitta and W. C. Marra, "Global undersea communication networks," IEEE Commun. Mag., vol. 34, no. 2, pp. 20-21, 1996.

[4] N. S. Bergano, Undersea Communication Systems. Academic Press, Optical Fiber Telecommunications IVB Components ed., 2002.

[5] ITU Recommendation, Gigabit-capable passive optical networks (GPON). ITU-T, 2003.

[6] IEEE P802.3av Task Force, IEEE Std 802.3av-2009 $10 \mathrm{~Gb} / \mathrm{s}$ Ethernet passive optical network. IEEE, New York.

[7] P. P. Iannone, K. C. Reichmann, C. R. Doerr, L. L. Buhl, M. A. Cappuzzo, E. Y. Chen, L. T. Gomez, J. E. Johnson, A. M. Kanan, J. L. Lentz, and R. McDonough, "A $40 \mathrm{~Gb} / \mathrm{s}$ CWDM-TDM PON with a cyclic CWDM multiplexer/demultiplexer," in 32nd European Conference on Optical Communications, ECOC'09, (Vienna, Austria), 2009. Paper 8.5.6. 
[8] P. J. Winzer, G. Raybon, and M. Duelk, "107-Gb/s optical ETDM transmission for $100 \mathrm{G}$ Ethernet transport," in 31st European Conference on Optical Communications, ECOC'05, (Glasgow, Scotland), 2005. Paper Th.4.1.1.

[9] IEEE P802.3ba 40Gb/s and 100Gb/s Ethernet Task Force, IEEE Std. 802.3ba-2010. IEEE, New York.

[10] D. van den Borne, S. L. Jansen, E. Gottwald, E. D. Schmidt, G. D. Khoe, and H. de Waardt, "DQPSK modulation for robust optical transmission," in Conference on Optical Fiber communication/National Fiber Optic Engineers Conference, 2008. OFC/NFOEC 2008., (San Diego, CA), 2008. Paper OMQ1.

[11] P. Boffi, M. Ferrario, L. Marazzi, P. Martelli, P. Parolari, A. Righetti, R. Siano, and M. Martinelli, "Stable 100-Gb/s POLMUX-DQPSK transmission with automatic polarization stabilization," IEEE Photon. Technol. Lett., vol. 21, no. 11, pp. 745-747, 2009.

[12] A. Sano, E. Yoshida, H. Masuda, T. Kobayashi, E. Yamada, Y. Miyamoto, F. Inuzuka, Y. Hibino, Y. Takatori, K. Hagimoto, T. Yamada, and Y. Sakamaki, "30 × 100-Gb/s all-optical OFDM transmission over $1300 \mathrm{~km}$ SMF with 10 ROADM nodes," in 33rd European Conference on Optical Communications, ECOC'07, (Berlin, Germany), 2007. Postdeadline Paper PD 1.7.

[13] S. Chandrasekhar and X. Liu, "Experimental investigation on the performance of closely spaced multi-carrier PDM-QPSK with digital coherent detection," Optics express, vol. 17, no. 24, pp. 21350213061, 2009.

[14] J. A. Wells, "Faster than fiber: the future of multi-Gb/s wireless," IEEE Microw. Mag., vol. 10, no. 3, pp. 104-112, 2009.

[15] P. Adhikari, "Understanding millimeter wave wireless communications." White paper, Loea Corporation, L1104-WP, June 2008. Available online.

[16] X. Hao, V. Kukshya, and T. S.Rappaport, "Spatial and temporal characteristics of $60-\mathrm{GHz}$ indoor channels," IEEE J. Sel. Areas Commun., vol. 20, no. 3, pp. 620-630, 2002. 
[17] T. H. Maiman, "Stimulated optical radiation in ruby," Nature, vol. 187, pp. 493-494, 1960.

[18] W. Stephens and T. Joseph, "System characteristics of direct modulated and externally modulated RF fiber-optic links," J. Lightw. Technol., vol. 5, no. 3, pp. 380-387, 1987.

[19] G. K. Gopalakrishnan, W. K. Burns, and C. H. Bulmer, "Microwave-optical mixing in $\mathrm{LiNbO}_{3}$ modulators," IEEE Trans. Microw. Theory Tech., vol. 41, no. 12, pp. 2383-2391, 1993.

[20] C. Cox III, E. Ackerman, R. Helkey, and G. E. Betts, "Techniques and performance of intensity-modulation direct-detection analog optical links," IEEE Trans. Microw. Theory Tech., vol. 45, no. 8, pp. 1375-1383, 1997.

[21] T. Kurniawan, A. Nirmalathas, C. Lim, D. Novak, and R. Waterhouse, "Performance analysis of optimized millimeter-wave fiber radio links," IEEE Trans. Microw. Theory Tech., vol. 54, no. 2, pp. 921-928, 2006.

[22] A. J. Seeds, C. H. Lee, E. Funk, and M. Nagamura, "Guest editorial: Microwave photonics," IEEE Photon. Technol. Lett., vol. 21, no. 12 , pp. 2959-2961, 2003.

[23] A. J. Seeds and K. J. Williams, "Microwave photonics," J. Lightw. Technol., vol. 24, no. 12, pp. 4628-4641, 2006.

[24] D. Capmany, J. Novak, "Microwave photonics combines two worlds," Nature Photonics, vol. 1, pp. 319-330, 2007.

[25] C. H. Cox III and E. I. Ackerman, "Microwave photonics: Past, present and future," in 2008 International Topical Meeting on Microwave Photonics jointly held with the 2008 Asia-Pacific Microwave Photonics Conference, (Gold Coast, Australia), 2008.

[26] E. I. Ackerman, W. K. Burns, G. E. Betts, J. X. Chen, J. L. Prince, M. D. Regan, H. V. Roussell, and C. H. Cox III, "RF-Over-Fiber Links With Very Low Noise Figure," J. Lightw. Technol., vol. 26, no. 15 , pp. 2441-2448, 2008.

[27] E. I. Ackerman and C. H. Cox III, "RF Fiber-optic link performance," IEEE Microw. Mag., vol. 2, no. 4, pp. 50-58, 2001. 
[28] S. Hunziker and W. Baechtold, "Cellular remote antenna feeding: optical fibre or coaxial cable?," Electron. Lett., vol. 34, no. 11, pp. 1038-1040, 1998.

[29] R. E. Schuh, A. Alping, and E. Sundberg, "Penalty-free GSM-1800 and WCDMA radio-over-fibre transmission using multimode fibre and $850 \mathrm{~nm}$ VECSEL," Electron. Lett., vol. 39, no. 6, pp. 512-514, 2003.

[30] M. Sauer, A. Kobyakov, and J. George, "Radio over fiber for picocellular network architectures," J. Lightw. Technol., vol. 25, no. 11, pp. 3301-3320, 2007.

[31] T. E. Darcie, "Subcarrier multiplexing for lightwave networks and video distribution systems," IEEE J. Sel. Areas Commun., vol. 8, pp. 1240-1248, 1990.

[32] P. M. Hill and R. Olshansky, "A 20-channel optical communication using subcarrier multiplexing for the transmission of digital video signals," J. Lightw. Technol., vol. 8, pp. 554-560, 1990.

[33] D. Nadir, "Wide-bandwidth lasers and modulators for RF photonics," IEEE Trans. Microw. Theory Tech., vol. 47, no. 7, pp. 11511171, 1999.

[34] C. B. Su, V. Lanzisera, W. Powazinik, E. Meland, R. Olshansky, and R. B. Lauer, "12.5-GHz direct modulation bandwidth of vapor phase regrown $1.3-\mu \mathrm{m}$ InGaAsP buried heterostructure lasers," Appl. Phy. Lett., vol. 46, no. 4, pp. 344-346, 1985.

[35] J. Bowers, U. Koren, B. Miller, C. Soccolich, and W. Jan, "Highspeed, polyimide-based semi-insulating planar buried heterostructures," Electron. Lett., vol. 23, no. 24, pp. 1263-1264, 1987.

[36] D. Klotzkin, K. Kamath, and P. Bhattacharya, "Quantum capture times at room temperature in high-speed $\mathrm{In}_{0.4} \mathrm{Ga}_{0.6}$ As-GaAs self-organized quantum-dot lasers," IEEE Photon. Technol. Lett., vol. 9, no. 10, pp. 1301-1303, 1997.

[37] K. Uomi, H. Nakano, and N. Chinone, "Ultrahigh-speed $1.55 \mu \mathrm{m}$ $\lambda / 4$-shifted DFB PIQ-BH lasers with bandwidth of $17 \mathrm{GHz}, "$ Electron. Lett., vol. 25, no. 10, pp. 668-669, 1989. 
[38] Y. Hirayama, M. Morinaga, M. Onomura, M. Tanimura, M. Tohyama, M. Funemizu, M. Kushibe, N. Suzuki, and M. Nakamura, "High-speed 1.5- $\mu \mathrm{m}$ compressively strained multi-quantum well self-aligned constricted mesa DFB lasers," J. Lightw. Technol., vol. 10, no. 9, pp. 1272-1280, 1992.

[39] P. Morton, T. Tanbun-Ek, R. Logan, J. Sciortino, P.F., A. Sergent, and K. Wecht, "Superfast $1.55 \mu \mathrm{m}$ DFB lasers," Electon. Lett., vol. 29, no. 16, pp. 1429-1430, 1993.

[40] S. Weisser, E. Larkins, K. Czotscher, W. Benz, J. Daleiden, I. Esquivias, J. Fleissner, J. Ralston, B. Romero, R. Sah, A. Schonfelder, and J. Rosenzweig, "Damping-limited modulation bandwidths up to $40 \mathrm{GHz}$ in undoped short-cavity $\mathrm{In}_{0.35} \mathrm{Ga}_{0.65} \mathrm{As}$-GaAs multiplequantum-well lasers," IEEE Photon. Technol. Lett., vol. 8, no. 5, pp. 608-610, 1996.

[41] I. P. Kaminow and E. H. Turner, "Electrooptic light modulators," Appl. Optics., vol. 5, no. 10, pp. 1612-1620, 1966.

[42] I. P. Kaminow, V. Ramaswamy, R. V. Schmidt, and E. H. Turner, "Lithium niobate ridge waveguide modulator," IEEE J. Quantum Electron., vol. 10, no. 9, pp. 731-731, 1974.

[43] G. Gopalakrishnan, C. Bulmer, W. Burns, R. McElhanon, and A. Greenblatt, " $40 \mathrm{GHz}$, low half-wave voltage $\mathrm{Ti}: \mathrm{LiNbO}_{3}$ intensity modulator," Electron. Lett., vol. 28, no. 9, pp. 826-827, 1992.

[44] D. Dolfi and T. Ranganath, " $50 \mathrm{GHz}$ velocity-matched broad wavelength $\mathrm{LiNbO}_{3}$ modulator with multimode active section," Electron. Lett., vol. 28, no. 13, pp. 1197-1198, 1992.

[45] K. Noguchi, H. Miyazawa, and O. Mitomi, "75 GHz broadband Ti:LiNbO 3 optical modulator with ridge structure," Electron. Lett., vol. 30, no. 12, pp. 949-951, 1994.

[46] K. Noguchi, O. Mitomi, and H. Miyazawa, "Millimeter-wave Ti:LiNbO 3 optical modulators," J. Lightw. Technol., vol. 16, no. 4, pp. 615-619, 1998.

[47] E. Wooten, K. Kissa, A. Yi-Yan, E. Murphy, D. Lafaw, P. Hallemeier, D. Maack, D. Attanasio, D. Fritz, G. McBrien, and D. Bossi, 
"A review of lithium niobate modulators for fiber-optic communications systems," IEEE J. Sel. Topics Quantum Electron., vol. 6, no. 1, pp. 69-82, 2000.

[48] M. Leeson, F. Payne, R. Mears, J. Carroll, J. Roberts, M. Pate, and G. Hill, "Design and fabrication of planar, resonant Franz-Keldysh optical modulator," Electron. Lett., vol. 24, no. 25, pp. 1546-1547, 1988.

[49] D. A. B. Miller, D. S. Chemla, T. C. Damen, A. C. Gossard, W. Wiegmann, T. H. Wood, and C. A. Burrus, "Electric field dependence of optical absorption near the band gap of quantum-well structures," Phys. Rev. B, vol. 32, no. 2, pp. 1043-1060, 1985.

[50] C. Zhang, Y.-J. Chiu, P. Abraham, and J. Bowers, "25 $\mathrm{GHz}$ polarization-insensitive electroabsorption modulators with traveling-wave electrodes," IEEE Photon. Technol. Lett., vol. 11, no. 2, pp. 191-193, 1999.

[51] M. Chacinski, U. Westergren, B. Stoltz, and L. Thylen, "Monolithically Integrated DFB-EA for $100 \mathrm{~Gb} / \mathrm{s}$ Ethernet," IEEE Electron Device Lett., vol. 29, no. 12, pp. 1312-1314, 2008.

[52] T. Ishibashi, N. Shimizu, S. Kodama, H. Ito, T. Nagatsuma, and T. Furuta, "Uni-travelling carrier photodiodes," in Ultrafast Electronics and Optoelectronics, (Incline Village, NV.), pp. 83-87, 1997.

[53] M. Gustavsson, P. O. Hedekvist, and P. A. Anderkson, "Unitravelling-carrier photodioide performance with $\mathrm{x}$-band modulation at high optical power," IEEE Microw. Wireless Compon. Lett., vol. 15, no. 5, pp. 297-299, 2005.

[54] H. Ito, F. Nakajima, T. Furuta, and T. Ishibashi, "Continuous THzwave generation using antenna-integrated uni-travelling-carrier photodiodes," Semiconductor Science and Technology, vol. 20, no. 7, pp. S191-S198, 2005.

[55] R. Alemany, J. Perez, R. Llorente, V. Polo, and J. Marti, "Coexistence of WiMAX 802.16d and MB-OFDM UWB in radio over multi-mode fiber indoor systems," in International Topicsl Meeting on Microwave Photonics, jointly held with the 2008 Asia- 
Pacific Microwave Photonics Conference. MWP/APMP 2008, (GoldCoast, Australia), pp. 74-77, 2008.

[56] D. Wake, D. Johansson, and D. Moodie, "Passive picocell: a new concept in wireless network architecture," Electron. Lett., vol. 33, no. 5, pp. 404-406, 1997.

[57] K. Kojucharow, M. Sauer, H. Kaluzni, D. Sommer, F. Poegel, W. Nowak, a. Finger, and D. Ferling, "Simultaneous electrooptical upconversion, remote oscillator generation, and air transmission of multiple optical WDM channels for a $60-\mathrm{GHz}$ high-capacity indoor system," IEEE Trans. Microw. Theory Tech., vol. 47, no. 12, pp. 2249-2256, 1999.

[58] T. Kuri, J. Vegas-Olmos, H. Toda, and K.-I. Kitayama, "Wired and wireless integrated DWDM networks and millimeter-waveband RoF technologies," in IEEE LEOS Annual Meeting Conference Proceedings, 2009. LEOS '09., pp. 677-678, oct. 2009.

[59] A. Chowdhury, H.-C. Chien, Y.-T. Hsueh, and G.-K. Chang, "Advanced system technologies and field demonstration for in-building optical-wireless network with integrated broadband services," $J$. Lightw. Technol., vol. 27, no. 12, pp. 1920-1927, 2009.

[60] J. Fuster, J. Marti, P. Candelas, F. J. Martinez, and L. Semprere, "Optical generation of electrical modulation formats," in 27th European Conference on Optical Communications, ECOC'01, (Amsterdam, Netherlands), 2001. Paper Th. B.1.7.

[61] A. Loayssa, D. Benito, and M. J. Garder, "High-bit rate all-optical BPSK modulator for fiber-optic microwave/millimeter-wave links," in 14th Annual Meeting of IEEE Laser and Electro-optics Society, LEOS'01, (San Diego, CA.), pp. 316-317.

[62] S. Chandramouli, W. Jimison, and E. Funk, "Direct carrier modulation for wireless digital communications using an improved microwave-photonic vector modulator (MPVM) approach," 2002 IEEE MTT-S International Microwave Symposium Digest (Cat. No.02CH37278), pp. 1293-1296, 2002. 
[63] A. Hirata, M. Harada, and T. Nagatsuma, "Multi-gigabit/s wireless links using millimeter-wave photonic techniques," in $2001 \mathrm{In}$ ternational Topical Meeting on Microwave Photonics. Tech. Dig., MWP'01 (Cat. No.01EX476), (Long Beach, CA), pp. 77-80, 2002.

[64] A. Loayssa and D. Benito, "All-optical implementation of broadband QPSK subcarrier modulator," Electron. Lett., vol. 40, no. 21, pp. 2-3, 2004.

[65] A. Hirata, T. Kosugi, H. Takahashi, R. Yamaguchi, F. Nakajima, T. Furuta, H. Ito, H. Sugahara, Y. Sato, and T. Nagatsuma, "120GHz-band millimeter-wave photonic wireless link for 10-Gb/s data transmission," IEEE Trans. Microw. Theory Tech., vol. 54, no. 5, pp. 1937-1944, 2006.

[66] M. Weiss, M. Huchard, A. Stohr, B. Charbonnier, S. Fedderwitz, and D. Jager, "60-GHz photonic millimeter-wave link for short-to medium-range wireless transmission up to $12.5 \mathrm{~Gb} / \mathrm{s}$," J. Lightw. Technol., vol. 26, no. 15, pp. 2424-2429, 2008.

[67] A. Ng'oma, M. Sauer, J. George, and D. Thelen, "Bit-rate doubling in multi-Gbps wideband ASK-modulated $60 \mathrm{GHz}$ RoF links using linear feed-forward equalisation and direct conversion transceivers," in 34th European Conference on Optical Communications, ECOC'08, (Brussels, Belgium), 2008. Paper P 6.12.

[68] P. Smulders, "Exploiting the $60 \mathrm{GHz}$ band for local wireless multimedia access: prospects and future directions," IEEE Commun. Mag., vol. 40, no. 1, pp. 140-147, 2002.

[69] S. Reynolds, B. Floyd, U. Pfeiffer, T. Beukema, T. Zwick, J. Grzyb, D. Liu, and B. Gaucher, "Progress toward a low-cost millimeterwave Silicon radio," in IEEE Custom Integrated Circuits Conference, 2005, pp. 563-570, 2005.

[70] J. Yu, J. Hu, D. Qian, Z. Jia, G. K. Chang, and T. Wang, "Transmission of microwave-photonics generated 16Gbit/s super broadband OFDM signals in radio-over-fiber system," in Conference on Optical Fiber communication/National Fiber Optic Engineers Conference, 2008. OFC/NFOEC 2008., 2008. Paper OThP2. 
[71] C.-T. Lin, E.-Z. Wong, W.-J. Jiang, P.-T. Shin, J. Chen, and S. Chi, "28-Gb/s 16-QAM OFDM radio-over-fiber system within 7-GHz license-free band at $60 \mathrm{GHz}$ employing all-optical up-conversion," in Conference on Lasers and Electro-Optics, 2009 and 2009 Conference on Quantum electronics and Laser Science CLEO/QELS 2009., 2009. Postdeadline Paper CPDA8.

[72] M. Weiss, A. Stohr, F. Lecoche, and B. Charbonnier, "27 gbit/s photonic wireless $60 \mathrm{ghz}$ transmission system using 16-QAM OFDM," in Microwave Photonics, 2009. MWP '09. International Topical Meeting on. PostDeadline Paper.

[73] A. Ng'oma, P.-T. Shih, J. George, F. Annunziata, M. Sauer, C.-T. Lin, W.-J. Jiang, J. Chen, and S. Chi, "21 Gbps OFDM wireless signal transmission at $60 \mathrm{GHz}$ using a simple IMDD radio-overfiber system," in 2010 Conference on Optical Fiber Communication (OFC), collocated National Fiber Optic Engineers Conference, (OFC/NFOEC), 2010. paper OTuF4.

[74] R. P. Braun, G. Grosskopf, D. Rohde, and F. Schmidt, "Low-phase noise millimeter-wave generation at $64 \mathrm{GHz}$ and data transmission using optical sideband injection locking," IEEE Photon. Technol. Lett., vol. 10, no. 5, pp. 728-730, 1998.

[75] L. N. Langley, M. D. Elkin, C. Edge, M. J. Wale, U. Gliese, X. Huang, and A. J. Seeda, "Packaged semiconductor laser optical phase-locked loop OPLL for photonic generation, processing and transmission of microwave signals," IEEE Trans. Microw. Theory Tech., vol. 47, no. 7, pp. 1257-1264, 1999.

[76] Y. Li, M. Bystrom, D. Yoo, S. Goldwasser, and P. Herczfeld, "Coherent optical vector modulation for fiber radio using electrooptic microchip lasers," IEEE Trans. Microw. Theory Tech., vol. 53, no. 10, pp. 3121-3129, 2005.

[77] L. A. Johansson and A. J. Seeds, "36-GHz $140-\mathrm{Mb} / \mathrm{s}$ radioover-fiber transmission using an optical injection phase-lock loop source," IEEE Photon. Technol. Lett., vol. 13, no. 8, pp. 893-895, 2001.

[78] J. Downie, L. Tomkos, N. Antoniades, and A. Boskovic, "Effects of filter concatenation for directly modulated transmission lasers at 
2.5 and $10 \mathrm{~Gb} / \mathrm{s}$," J. Lightw. Technol., vol. 20, no. 2, pp. 218-228, 2002 .

[79] B. Dagens, A. Martinez, D. Make, O. L. Gouezigou, J.-G. Provost, V. Sallet, K. Merghem, J.-C. Harmand, A. Ramdane, and B. Thedrez, "Floor free $10-\mathrm{Gb} / \mathrm{s}$ transmission with directly modulated GaInNAs-GaAs 1.35- $\mu \mathrm{m}$ laser for metropolitan applications," IEEE Photon. Technol. Lett., vol. 17, no. 5, pp. 971-973, 2005.

[80] F. Koyama and K. Iga, "Frequency chirping in external modulators," J. Lightw. Technol., vol. 6, no. 1, pp. 87-93, 1988.

[81] T. Koch and J. Bowers, "Nature of wavelength chirping in directly modulated semiconductor lasers," Electron. Lett., vol. 20, no. 25, pp. 1038-1040, 1984.

[82] L. Bjerkan, A. Royset, L. Hafskjaer, and D. Myhre, "Measurement of laser parameters for simulation of high-speed fiberoptic systems," J. Lightw. Technol., vol. 14, no. 5, pp. 839-850, 1996.

[83] S. Walkin and J. Conradi, "Effect of MachZehnder modulator DC extinction ratio on residual chirp-induced dispersion in 10-Gb/s binary and AM-PSK duobinary lightwave systems," IEEE Photon. Technol. Lett., vol. 9, no. 10, pp. 1400-1403, 1997.

[84] H. Kim and A. Gnauck, "Chirp characteristics of dual-drive MachZehnder modulator with a finite DC extinction ratio," IEEE Photon. Technol. Lett., vol. 14, no. 3, pp. 298-300, 2002.

[85] S. Kaneko, M. Noda, Y. Miyazaki, H. Watanabe, K. Kasahara, and T. Tajime, "An electroabsorption modulator module for digital and analog applications," J. Lightw. Technol., vol. 17, no. 4, pp. 669676, 1999.

[86] W.-J. Choi, A. Bond, J. Kim, J. Zhang, R. Jambunathan, H. Foulk, S. O'Brien, J. Van Norman, D. Vandegrift, C. Wanamaker, J. Shakespeare, and H. Cao, "Low insertion loss and low dispersion penalty InGaAsP quantum-well high-speed electroabsorption modulator for $40-\mathrm{Gb} / \mathrm{s}$ very-short-reach, long-reach, and long-haul applications," J. Lightw. Technol., vol. 20, no. 12, pp. 2052-2056, 2002. 
[87] T. Kuri, K. Kitayama, and Y. Takahashi, "A single light-source configuration for full-duplex 60-GHz-band radio-on-fiber system," IEEE Trans. Microw. Theory Tech., vol. 51, pp. 431-439, feb. 2003.

[88] J. Kim, Y.-S. Kang, Y.-D. Chung, and K.-S. Choi, "Development and RF characteristics of analog 60-GHz electroabsorption modulator module for RF/optic conversion," IEEE Trans. Microw. Theory Tech., vol. 54, no. 2, pp. 780-787, 2006.

[89] R. Lewen, S. Irmscher, U. Westergren, L. Thylen, and U. Eriksson, "Segmented transmission-line electroabsorption modulators," J. Lightw. Technol., vol. 22, no. 1, pp. 172-179, 2004.

[90] G. P. Agrawal, Fiber-Optic Communication Systems. New York: Wiley Interscience, second ed., 1997.

[91] M. Mohamed, X. Zhang, B. Hraimel, and K. Wu, "Analysis of frequency quadrupling using a single Mach-Zehnder modulator for millimeter-wave generation and distribution over fiber systems," Opt. Express, vol. 16, no. 14, pp. 10786-10802, 2008.

[92] P.-T. Shih, C.-T. Lin, W.-J. Jiang, J. J. Chen, H.-S. Huang, Y.H. Chen, P.-C. Peng, and S. Chi, "WDM up-conversion employing frequency quadrupling in optical modulator," Opt. Express, vol. 17, no. 3, pp. 1726-1733, 2009.

[93] J. L. Corral, J. Marti, and J. M. Fuster, "General expressions for IM/DD dispersive analog optical links with external modulation or optical up-conversion in a MachZehnder electrooptical modulator," IEEE Trans. Microw. Theory Tech., vol. 49, no. 10, pp. 1968-1976, 2001.

[94] G. H. Smith, D. Novak, and Z. Ahmed, "Overcoming chromaticdispersion effects in fiber-wireless systems incorporating external modulators," IEEE Trans. Microw. Theory Tech., vol. 45, no. 8, pp. 1410-1415, 1997.

[95] J. Fuster, J. Marti, J. Corral, V. Polo, and F. Ramos, "Generalized study of dispersion-induced power penalty mitigation techniques in millimeter-wave fiber-optic links," J. Lightw. Technol., vol. 18, no. 7, pp. 933-940, 2000. 
[96] O. Mitomi, S. Nojima, I. Kotaka, K. Wakita, K. Kawano, and M. Naganuma, "Chirping characteristic and frequency response of MQW optical intensity modulator," J. Lightw. Technol., vol. 10, no. 1, pp. 71-77, 1992.

[97] Z. Jia, J. Yu, and G.-K. Chang, "A full-duplex radio-over-fiber system based on optical carrier suppression and reuse," J. Lightw. Technol., vol. 18, no. 16, pp. 1726-1728, 2006.

[98] A. Stohr, K. Kitayama, and D. Jager, "Full-duplex fiberoptic RF subcarrier transmission using a dual-function modulator/photodetector," IEEE Trans. Microw. Theory Tech., vol. 47, no. 7, pp. 1338-1341, 1999.

[99] T. Kuri and K. Kitayama, "Novel photonic downconversion technique with optical frequency shift for millimeter-wave-band radioon-fiber systems," IEEE Photon. Technol. Lett., vol. 14, no. 8, pp. 1163-1165, 2002.

[100] T. Kuri, K. Kitayama, and Y. Ogawa, "Fiber-optic millimeterwave uplink system incorporating remotely fed 60-GHz-band optical pilot tone," IEEE Trans. Microw. Theory Tech., vol. 47, no. 7, pp. 1332-1337, 1999.

[101] F. Giannetti, M. Luise, and Reggiannini, "Mobile and personal communications in the $60 \mathrm{GHz}$ band: a survey," Wireless Person. Comm., vol. 10, pp. 207-243, 1999.

[102] H. Schmuck, R. Heidemann, and R. Hofstetter, "Distribution of 60GHz signals to more than 1000 base stations," Electron. Lett., vol. 30, no. 1, pp. 59-60, 1994.

[103] K. K. M. Sauer, H. Kaluzni, D. Sommer, F. Poegel, W. Nowak, A. Finger, and D. Ferling, "Simultaneous electrooptical upconversion, remote oscillator generation and air transmission of multiple optical WDM channels for a $60 \mathrm{GHz}$ high-capacity indoor system," IEEE Trans. Microw. Theory Tech., vol. 47, no. 12, pp. 2249-2256, 1999.

[104] C. S. Park, C. G. Oh, C. G. Lee, D. H. Kim, and C.-S. Park, "A photonic up-converter for a WDM radio-over-fiber system using 
cross-absorption modulation in an EAM," IEEE Photon. Technol. Lett., vol. 17, no. 9, pp. 1950-1952, 2005.

[105] C. Lim, A. Nirmalathas, D. Novak, and R. Waterhouse, "Capacity analysis for WDM fiber-radio backbones with star-tree and ring architecture incorporating wavelength interleaving," J. Lightw. Technol., vol. 21, no. 12, pp. 3308-3315, 2003.

[106] H. Toda, T. Yamashita, T. Kuri, and K. Kitayama, "Demultiplexing using an arrayed-waveguide grating for frequency-interleaved DWDM millimeter-wave radio-on-fiber systems," J. Lightw. Technol., vol. 21, no. 8, pp. 1735-1741, 2003.

[107] T. Kuri, H. Toda, and K. Kitayama, "Novel demultiplexer for DWDM $60-\mathrm{GHz}$ millimeter-wave-band radio-over-fiber systems with optical frequency interleaving techniques," IEEE Photon. Technol. Lett., vol. 19, no. 24, pp. 2018-2020, 2007.

[108] H. Takara, T. Ohara, K. Mori, K. Sato, E. Yamada, Y. Inoue, T. Shibata, A. M., T. Morioka, and K. I. Sato, "More than 1000 channel optical frequency chain generation from single supercontinuum source with $12.5 \mathrm{GHz}$ channel spacing," Electron. Lett., vol. 26, no. 25, pp. 2089-2090, 2000.

[109] T. Nakasyotani, H. Toda, T. Kuri, and K. Kitayama, "Wavelengthdivision-multiplexed millimeter-wave-band radio-on-fiber system using a supercontinuum light source," J. Lightw. Technol., vol. 24, no. 1, pp. 404-410, 2005.

[110] J. Zia, J. Yu, A. Chowdhury, G. Ellinas, and G. K. Chang, "Simultaneous generation of independent wired wireless services using a single modulator in millimeter-wave band radio-over-fiber systems," IEEE Photon. Technol. Lett., vol. 19, no. 20, pp. 1691-1693, 2007.

[111] H. S. Kim, T. T. Pham, Y. Y. Won, and S. K. Han, "Simultaneous wired and wireless 1.25-Gb/s bidirectional WDM-RoF transmission using multiple optical carrier suppression in FP LD," J. Lightw. Technol., vol. 27, no. 14, pp. 2744-2750, 2009. 
[112] J. J. Vegas-Olmos, T. Kuri, and K. Kitayama, "Simultaneous wired and wireless $1.25-\mathrm{Gb} / \mathrm{s}$ bidirectional WDM-RoF transmission using multiple optical carrier suppression in FP LD," IEEE Photon. Technol. Lett., vol. 20, no. 8, pp. 617-619, 2008.

[113] J. J. Vegas-Olmos, T. Kuri, and K. ichi Kitayama, "Dynamic reconfigurable WDM 60-GHz millimeter-waveband radio-over-fiber access network: Architectural considerations and experiment," $J$. Lightw. Technol., vol. 25, no. 11, pp. 3374-3380, 2007.

[114] B. Ortega, J. Mora, G. Puerto, and J. Capmany, "Symmetric reconfigurable capacity assignment in a bidirectional DWDM access network," Opt. Express, vol. 15, no. 25, pp. 16781-16786.

[115] Y. Doi, S. Fukushima, T. Ohno, Y. Matsuoka, and H. Takeuchi, "Phase shift keying using optical delay modulation for millimeterwave fiber-optic radio links," J. Lightw. Technol., vol. 18, no. 3, pp. 301-307, 2000.

[116] A. H. Gnauck and P. J. Winzer, "Optical phase-shift-keyed transmission," J. Lightw. Technol., vol. 23, no. 1, pp. 115-130, 2005.

[117] L. G. Kazovsky, G. Kalogerakis, and W.-T. Shaw, "Homodyne Phase-Shift-Keying Systems: Past Challenges and Future Opportunities," J. Lightw. Technol., vol. 24, no. 12, pp. 4876-4884, 2006.

[118] L. Kazovsky and D. Atlas, "A 1320-nm experimental optical phaselocked loop: performance investigation and PSK homodyne experiments at $140 \mathrm{Mb} / \mathrm{s}$ and $2 \mathrm{~Gb} / \mathrm{s}$," J. Lightw. Technol., vol. 8, no. 9, pp. 1414-1425, 1990.

[119] J. Kahn, A. Gnauck, J. Veselka, S. Korotky, and B. Kasper, "4$\mathrm{Gb} / \mathrm{s}$ PSK homodyne transmission system using phase-locked semiconductor lasers," IEEE Photon. Technol. Lett., vol. 2, no. 4, pp. 285-287, 1990.

[120] R. Vodhanel, "5 Gbit/s direct optical DPSK modulation of a 1530nm DFB laser," IEEE Photon. Technol. Lett., vol. 1, no. 8, pp. 218$220,1989$.

[121] B. Zhu, L. Leng, A. H. Gbauck, M. O. Pedersen, D. Peckham, L. E. Nelson, S. Stulz, S. Kado, J. Gruner-Nielsen, L. J. R. L., 
S. Knudsen, J. Leuthold, C. Doerr, S. Chandrasekhar, G. Baynham, P. Gaarde, Y. Emori, and S. Namiki, "Transmission of 3.2 $\mathrm{Tb} / \mathrm{s}(80 \times 42.7 \mathrm{~Gb} / \mathrm{s})$ over $5200 \mathrm{~km}$ of Ultrawave ${ }^{T M}$ fiber with 100 $\mathrm{km}$ dispersion-managed spans using RZ-DPSK format," in 28th European Conference on Optical Communications, ECOC'02, (Copenhagen, Denmark), 2002. Postdeadline paper PD4.2.

[122] G. Charlet, E. Corbel, J. Lazaro, A. Klekamp, R. Dischler, P. Tran, W. Idler, H. Mardoyan, A. Konczykowska, F. Jorge, and S. Bigo, "WDM transmission at $6 \mathrm{Tbit} / \mathrm{s}$ capacity over transatlantic distance, using $42.7 \mathrm{~Gb} / \mathrm{s}$ differential phase-shift keying without pulse carver," in Optical Fiber Communication Conference, 2004. OFC 2004, (Los Angeles, CA), 2004. Postdeadline paper PD36.

[123] C. Wree, J. Leibrich, J. Eick, and W. Rosenkranz, "Experimental investigation of receiver sensitivity of RZ-DQPSK modulation format using balanced detection," in Optical Fiber Communication Conference, 2003. OFC 2003, (Atlanta, GA), 2003. Paper ThE5.

[124] J. Barry and J. Kahn, "Carrier synchronization for homodyne and heterodyne detection of optical quadriphase-shift keying," $J$. Lightw. Technol., vol. 10, no. 12, pp. 1939-1951, 1992.

[125] A. H. Gnauck, P. J. Winzer, S. Chandrasekhar, and C. Doerr, "Spectrally efficient $(0.8 \mathrm{~b} / \mathrm{s} / \mathrm{Hz}) \quad 1-\mathrm{Tb} / \mathrm{s} \quad(25 \times 42.7 \mathrm{~Gb} / \mathrm{s})$ RZ-DQPSK transmission over 28 100-km SSMF spans with 7 add/drops," in 30th European Conference on Optical Communications, ECOC'04, (Stockholm, Sweden). Postdeadline paper Th4.4.1.

[126] G. Charlet, P. Tran, H. Mardoyan, M. Lefrancois, T. Fauconnier, F. Jorge, and S. Bigo, "151 × $43 \mathrm{~Gb} / \mathrm{s}$ transmission over 4080 $\mathrm{km}$ based on return-to-zero-differential quadrature phase-shift keying," in 31st European Conference on Optical Communications, ECOC'05., 2005. Postdeadline paper Th4.1.3.

[127] C. Furst, J.-P. Elbers, H. Wernz, H. Griesser, S. Herbst, M. Camera, F. Cavaliere, A. Ehrhardt, D. Breuer, D. Fritzsche, S. Vorbeck, M. Schneiders, W. Weiershausen, R. Leppla, J. Wendler, M. Schrodel, T. Wuth, C. Fludger, T. Duthel, B. Milivojevic, 
and C. Schulien, "Analysis of crosstalk in mixed $43 \mathrm{~Gb} / \mathrm{s} \mathrm{RZ-}$ DQPSK and $10.7 \mathrm{~Gb} / \mathrm{s}$ DWDM systems at $50 \mathrm{GHz}$ channel spacing," in Conference on Optical Fiber Communication and the National Fiber Optic Engineers Conference, 2007. OFC/NFOEC 2007., 2007. paper OThS2.

[128] M. Daikoku, I. Morita, H. Taga, H. Tanaka, T. Kawanishi, T. Sakamoto, T. Miyazaki, and T. Fujita, "100-Gb/s DQPSK transmission experiment without OTDM for 100G Ethernet transport," J. Lightw. Technol., vol. 25, no. 1, pp. 139-145, 2007.

[129] P. Winzer, G. Raybon, H. Song, A. Adamiecki, S. Corteselli, A. Gnauck, D. Fishman, C. Doerr, S. Chandrasekhar, L. Buhl, T. Xia, G. Wellbrock, W. Lee, B. Basch, T. Kawanishi, K. Higuma, and Y. Painchaud, "100-Gb/s DQPSK transmission: From laboratory experiments to field trials," J. Lightw. Technol., vol. 26, no. 20, pp. 3388-3402, 2008.

[130] L. Marazzi, P. Parolari, P. Martelli, R. Siano, P. Boffi, M. Ferrario, A. Righetti, M. Martinelli, V. Pusino, P. Minzioni, I. Cristiani, V. Degiorgio, C. Langrock, and M. Fejer, "Real-time 100-Gb/s POLMUX RZ-DQPSK transmission over uncompensated $500 \mathrm{~km}$ of SSMF by optical phase conjugation," in Conference on Optical Fiber Communication - incudes post deadline papers, 2009. OFC 2009., (San Diego, CA), 2009.

[131] R. Griffin and A. Carter, "Optical differential quadrature phaseshift key (oDQPSK) for high capacity optical transmission," in Optical Fiber Communication Conference and Exhibit, 2002. OFC 2002, 2002. Paper WX6.

[132] C. Wree, J. Leibrich, and W. Rosenkranz, "RZ-DQPSK format with high spectral efficiency and high robustness towards fiber nonlinearities," in 28th European Conference on Optical Communications, ECOC'02, (Copenhagen, Denmark). Paper 9.6.6.

[133] C. Doerr, L. Zhang, P. Winzer, J. Sinsky, A. Adamiecki, N. Sauer, and G. Raybon, "Compact high-speed InP DQPSK modulator," IEEE Photon. Technol. Lett., vol. 19, no. 15, pp. 1184-1186, 2007. 
[134] M. Nakamura, Y. Kamio, and T. Miyazaki, "Linewidth-tolerant 10-Gbit/s 16-QAM transmission using a pilot-carrier based phasenoise cancelling technique," Opt. Express, vol. 16, no. 14, pp. 10611-10616, 2008.

[135] P. Winzer, A. Gnauck, C. Doerr, M. Magarini, and L. Buhl, "Spectrally efficient long-haul optical networking using 112-Gb/s polarization-multiplexed 16-QAM," J. Lightw. Technol., vol. 28, no. 4, pp. 547-556, 2010.

[136] R. Hofstetter, H. Schmuck, and R. Heidemann, "Dispersion effects in optical millimeter-wave systems using self-heterodyne method for transport and generation," IEEE Trans. Microw. Theory Tech., vol. 43, no. 9, pp. 2263-2269, 1995.

[137] Y. Ozeki, K. Higuma, S. Oikawa, M. Kishi, and M. Tsuchiya, "A 60-GHz optoelectronic mixing scheme of high image and carrier rejection ratios with an integrated optical single-sideband modulator employed," IEEE Trans. Microw. Theory Tech., vol. 49, no. 10, pp. 1986-1991, 2001.

[138] A. Wiberg, S. Member, P. Pérez-millán, M. V. Andrés, P. A. Andrekson, S. Member, and P. O. Hedekvist, "Fiber-optic 40-GHz mm-wave link with $2.5-\mathrm{Gb} / \mathrm{s}$ data transmission," IEEE Photon. Technol. Lett., vol. 17, no. 9, pp. 1938-1940, 2005.

[139] L. Liao, D. Samara-Rubio, M. Morse, A. Liu, D. Hodge, D. Rubin, U. Keil, and T. Franck, "High speed Silicon Mach-Zehnder modulator," Opt. express, vol. 13, no. 8, pp. 3129-3135, 2005.

[140] M. Lipson, "Compact Electro-Optic Modulators on a Silicon Chip," IEEE Journal of Selected Topics in Quantum Electronics, vol. 12, pp. 1520-1526, Nov. 2006.

[141] T. Pinguet, B. Analui, E. Balmater, D. Guckenberger, M. Harrison, R. Koumans, D. Kucharski, Y. Liang, G. Masini, A. Mekis, S. Mirsaidi, A. Narasimha, M. Peterson, D. Rines, V. Sadagopan, S. Sahni, T. Sleboda, D. Song, Y. Wang, B. Welch, J. Witzens, J. Yao, S. Abdalla, S. Gloeckner, and P. De Dobbelaere, "Monolithically integrated high-speed CMOS photonic transceivers," in 2008 5th IEEE International Conference on Group IV Photonics, pp. 362-364, sep. 2008. 
[142] S. J. Spector, M. W. Geis, G.-R. Zhou, M. E. Grein, F. Gan, M. A. Popovic, J. U. Yoon, D. M. Lennon, E. P. Ippen, F. Z. Kärtner, and T. M. Lyszczarz, "CMOS-compatible dual-output silicon modulator for analog signal processing," Opt. express, vol. 16, no. 15, pp. 11027-11031, 2008.

[143] A. Liu, L. Liao, Y. Chetrit, J. Basak, H. Nguyen, D. Rubin, and M. Paniccia, "Wavelength division multiplexing based photonic integrated circuits on Silicon-on-insulator platform," IEEE J. Sel. Topics Quantum Electron., vol. 16, no. 1, pp. 23-32, 2010.

[144] S. Manipatruni, L. Chen, and M. Lipson, "Ultra high bandwidth WDM using Silicon microring modulators.," Opt. express, vol. 18, no. 16, pp. 16858-16867, 2010.

[145] F. Vacondio, M. Mirshafiei, J. Basak, A. Liu, L. Liao, M. Paniccia, and L. A. Rusch, "A Silicon modulator enabling RF over fiber for 802.11 OFDM signals," IEEE J. Sel. Topics Quantum Electron., vol. 16, no. 1, pp. 141-148, 2010.

[146] L. Zhang, Y. Li, J.-Y. Yang, M. Song, R. G. Beausoleil, and A. E. Willner, "Silicon-based microring resonator modulators for intensity modulation," IEEE J. Sel. Topics Quantum Electron., vol. 16, no. 1, pp. 149-158, 2010.

[147] L. Liao, A. Liu, J. Basak, H. Nguyen, M. Paniccia, D. Rubin, Y. Chetrit, R. Cohen, and N. Izhaky, "40 Gbit/s Silicon optical modulator for highspeed applications," Electron. Lett., vol. 43, no. $22,2007$.

[148] P. Dong, R. Shafiiha, S. Liao, H. Liang, N.-N. Feng, D. Feng, G. Li, X. Zheng, A. V. Krishnamoorthy, and M. Asghari, "Wavelengthtunable silicon microring modulator," Opt. Express, vol. 18, no. 11, pp. 10941-10946, 2010.

[149] S. Norimatsu and K. Iwashita, "Damping factor influence on linewidth requirements for optical PSK coherent detection systems," J. Lightw. Technol., vol. 11, no. 7, pp. 1226-1233, 1993.

[150] S. Savory, "Coherent detection - why is it back?," in The 20th Annual Meeting of the IEEE Lasers and Electro-Optics Society, 200\%. LEOS 200\%., pp. 212-213, 2007. 
[151] P. Bayvel, C. Behrens, R. Killey, S. Makovejs, D. Millar, and S. Savory, "Coherent electronic compensation techniques for longhaul optical fibre transmission - opportunities and challenges," in 35th European Conference on Optical Communications, ECOC'09., 2009. Paper 10.7.2.

[152] S. Savory, G. Gavioli, R. Killey, and P. Bayvel, "Transmission of 42.8Gbit/s Polarization Multiplexed NRZ-QPSK over $6400 \mathrm{~km}$ of Standard Fiber with no Optical Dispersion Compensation," in Conference on Optical Fiber Communication and the National Fiber Optic Engineers Conference, 200\%. OFC/NFOEC 2007., 2007. Paper OTuA1.

[153] J. Renaudier, G. Charlet, M. Salsi, O. Pardo, H. Mardoyan, P. Tran, and S. Bigo, "Linear fiber impairments mitigation of 40Gbit/s polarization-multiplexed QPSK by digital processing in a coherent receiver," J. Lightw. Technol., vol. 26, no. 1, pp. 36-42, 2008 .

[154] J. K. Fischer, R. Ludwig, L. Molle, C. Schmidt-Langhorst, A. Galperin, T. Reichter, C. C. Leonhardt, A. Matiss, and C. Scheubert, "High-Speed Digital Coherent Receiver With Parallel Optical Processing," in 2010 Conference on Optical Fiber Communication (OFC) collocated National FIber Optic Enginerrs Conference (OFC/NFOEC 2010), (San Diego, CA), 2010. Postdeadline Paper PDPB6.

[155] C. R. Doerr, L. Zhang, and P. J. Winzer, "Monolithic InP Multiwavelength Coherent Receiver," in 2010 Conference on Optical Fiber Communication (OFC) collocated National FIber Optic Enginerrs Conference (OFC/NFOEC 2010), (San Diego, CA), 2010. Postdeadline Paper PDPB1.

[156] U. Mengali and A. N. D'Andrea, Synchronization techniques for Digital Receivers. New York: Plenum, 1997.

[157] M. G. Taylor, "Phase estimation methods for optical coherent detection using digital signal processing," J. Lightw. Technol., vol. 27, no. 7, pp. 901-914, 2009. 
[158] M. Taylor, "Coherent detection method using DSP for demodulation of signal and subsequent equalization of propagation impairments," IEEE Photon. Technol. Lett., vol. 16, no. 2, pp. 674-676, 2004.

[159] S. J. Savory, "Digital filters for coherent optical receivers," Opt. Express, vol. 16, no. 2, pp. 804-817, 2008.

[160] D. Godard, "Self-recovering equalization and carrier tracking in two-dimensional data communication systems," IEEE Trans. Commun., vol. 28, no. 11, pp. 1867-1875, 1980.

[161] S. J. Savory, G. Gavioli, R. I. Killey, and P. Bayvel, "Electronic compensation of chromatic dispersion using a digital coherent receiver," Opt. express, vol. 15, no. 5, pp. 2120-2126, 2007.

[162] R. Noé, "PLL-free synchronous QPSK polarization multiplex/diversity receiver concept with digital I\&Q baseband processing," IEEE Photon. Technol. Lett., vol. 17, no. 4, pp. 887-889, 2005.

[163] E. Ip and J. M. Kahn, "Feedforward carrier recovery for coherent optical communications," J. Lightw. Technol., vol. 25, no. 9, pp. 2675-2692, 2007.

[164] A. J. Viterbi and A. N. Viterbi, "Nonlinear estimation of PSKmodulated carrier phase with application to burst digital transmission," IEEE Trans. Inf. Theory, vol. 29, no. 4, pp. 543-551, 1983.

[165] A. Sano, E. Yamada, H. Masuda, E. Yamazaki, T. Kobayashi, E. Yoshida, Y. Miyamoto, R. Kudo, K. Ishihara, and Y. Takatori, "No-guard-interval coherent optical OFDM for 100-Gb/s long-haul WDM transmission," J. Lightw. Technol., vol. 27, no. 16, pp. 37053713, 2009. 


\section{Paper A}

Photonic Envelope Detector for Broadband Wireless Signals Using a Single Mach- Zehnder Modulator and a Fibre Bragg Grating

R. Sambaraju, M. A. Piqueras, V. Polo, J. L. Corral, and J. Martí

Presented at 33rd European Conference on Optical Communication, ECOC 2008, paper P6.04, Brussels Belgium, Sept. 2008. 


\title{
Photonic Envelope Detector for Broadband Wireless Signals using a Single Mach-Zehnder Modulator and a Fibre Bragg Grating
}

\author{
Rakesh Sambaraju, Jesus Palaci, Valentin Polo, and Juan Luis Corral \\ Valencia Nanophotonics Technology Center, Universidad Politecnica de Valencia, Camino de Vera S/N Ed IDI-5, \\ Accesso A, Valencia 46022, Spain. Email: rsambaraju@ntc.upv.es
}

\begin{abstract}
A novel technique for envelope detection of wireless signals using a single Mach-Zehnder modulator is presented. Experimental demonstration of $1.25 \mathrm{Gbit/s}$ ASK modulated $35 \mathrm{GHz}$ carrier envelope detection is presented.
\end{abstract}

\section{Introduction}

The increased demand in wireless transmission of broadband data has pushed research efforts towards the generation and detection of wireless signals at millimetre wave frequencies with data rates of some Gbit/s, using traditional photonic mixing arrangements $[1,2]$. Application of such wireless $\mathrm{Gb} / \mathrm{s}$ capable radio over fibre (RoF) systems may be for instance wireless extension and bridging of FTTH networks.

Recently, the generation of more spectrally efficient modulation formats like MQAM at $40 / 60 \mathrm{GHz}$ band have been reported $[3,4]$. When operation at higher frequency bands such as $94 \mathrm{GHz}$ or $120 \mathrm{GHz}$ is achieved, simpler bandwidth inefficient modulation formats like amplitude shift keying (ASK) can be used, due to the huge available bandwidth. To demodulate an ASK modulated carrier, envelope detection is needed. Recently envelope detection schemes have been proposed like for demodulating $10 \mathrm{Gbit} / \mathrm{s}$ ASK modulated $120 \mathrm{GHz}$ carrier [1] using an electrical envelope detector and later performing the E/O conversion, or using an electro-absorption modulator (EAM) biased at its inflection point and external optical injection of a DFB laser [5], both of which involve complicated electronics and optical devices. In this paper a simplified photonic envelope detector with a single Mach-Zehnder modulator and a fibre Bragg grating is proposed. The photonic envelope detector converts an ASK modulated wireless carrier into an ASK modulated optical carrier for feeding into an optical network. Experimental demonstration of envelope detection of $1.25 \mathrm{Gbit} / \mathrm{s}$ ASK modulated $35 \mathrm{GHz}$ carrier is presented, and an error free detection obtained. The choice of 1.25 Gbit/s, and $35 \mathrm{GHz}$ of carrier were determined and limited by the electrical mixer and amplifiers, and not the actual principle of operation.

\section{Working Principle}

A photonic envelope detector should be able to convert the amplitude modulated RF signals to optical ASK signals. In the proposed photonic envelope detector, the output of a CW light source is modulated with the amplitude modulated RF carrier using a
Mach-Zehnder modulator biased at its minimum transmission point. The output of the MZM is an optical carrier with the electrical signal half wave rectified, modulated on it as shown in Fig. 1.

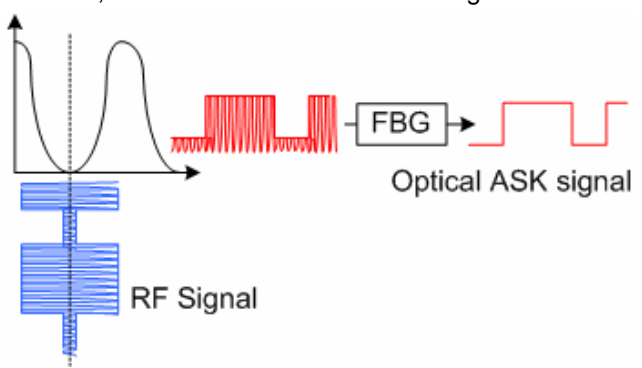

Figure 1: Principle of photonic envelope detection The output of the MZM contains two sidebands separated by $f_{L O}$, with the baseband data ASK modulated on each of them. Later, the output of the modulator is passed through a fibre Bragg grating, and only one sideband is selected. It should be noted that the bandwidth of the FBG should be more than twice the RF data rate. The optical carrier output of the FBG contains the data amplitude modulated on it.

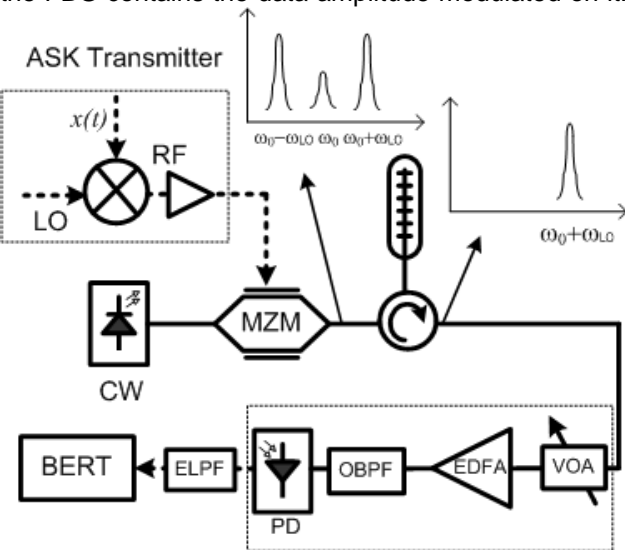

Figure 2: Schematic of the photonic envelope detector experimental setup.

\section{Experimental Setup}

Fig. 2 shows the schematic of the experimental setup used for the photonic envelope detector. It consists of 
three blocks: the electrical ASK modulated carrier generation, the optical envelope detector, and the optical pre-amplified receiver for measuring the bit error rate. The electrical signal was generated by mixing a $1.25 \mathrm{Gbit} / \mathrm{s} 2^{31}-1$ PRBS data, $x(t)$, with a 35 $\mathrm{GHz}$ LO carrier. The output of the mixer was amplified to $+13 \mathrm{dBm}$ using an electrical amplifier prior to modulating the optical carrier. In the envelope detector, a continuous wave laser source at 1549.68 $\mathrm{nm}$ with an output power of $+11 \mathrm{dBm}$ was externally modulated by the $1.25 \mathrm{Gbit} / \mathrm{s}$ ASK $35 \mathrm{GHz}$ carrier using a $50 \mathrm{GHz}$ bandwidth MZM, biased at its minimum transmission point.
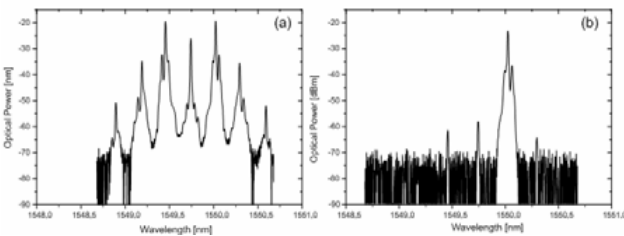

Figure 3: The optical spectrum at the output of the Mach-Zender modulator (a), at the output of the fibre Bragg grating (b).

The optical spectrum of the MZM output is shown in Fig. 3(a). A fibre Bragg grating was used with a circulator for filtering out the desired optical sideband. A $25 \mathrm{GHz}$ bandwidth FBG centred at $1550 \mathrm{~nm}$ was used for filtering the upper sideband. The output of the filter is a baseband data ASK modulated optical carrier at $1550 \mathrm{~nm}$. The optical spectrum of the ASK signal is shown in Fig. 3(b).

To measure the bit error ratio curve of the optical ASK signal a typical preamplified receiver was used. The output of the FBG was passed through an erbium doped fibre amplifier (EDFA) and followed by a tuneable band pass filter centred at $1550 \mathrm{~nm}$ with a bandwidth of $1 \mathrm{~nm}$ for filtering the accumulated stimulated emission (ASE) noise of the EDFA. A 12.5 Gbit/s photodiode was used to convert the optical signal to the electrical domain. A $900 \mathrm{MHz}$ low pass filter was used to filter the received data prior to bit error ratio test.

\section{Results}

Fig. 4 shows the BER curve plotted against the average optical power received to the pre-amplified receiver. Error free detection was obtained with received power as low as $-22 \mathrm{dBm}$. For a BER of $10^{-9}$, a received optical power of $-23.7 \mathrm{dBm}$ was required. One of the challenges faced was the high $\vee \pi$ of the MZM and not so high output power of the electrical amplifier. As an example to drive the MZM with full cycle a $24 \mathrm{dBm}$ RF signal is required, and the amplifiers available in the lab can only generate +13
$\mathrm{dBm}$ of power. The receiver sensitivity can be fairly improved by using a high power amplifier.

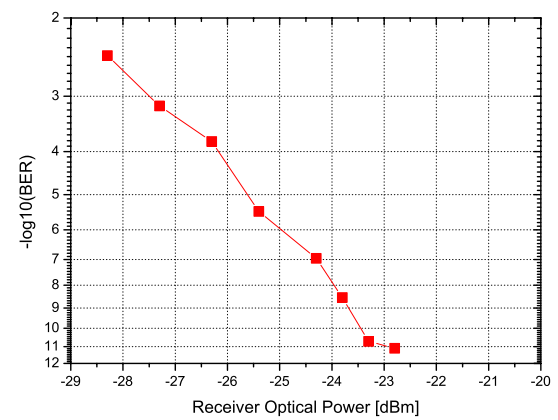

Figure 4: Measured bit error ratio plotted against the average received optical power.

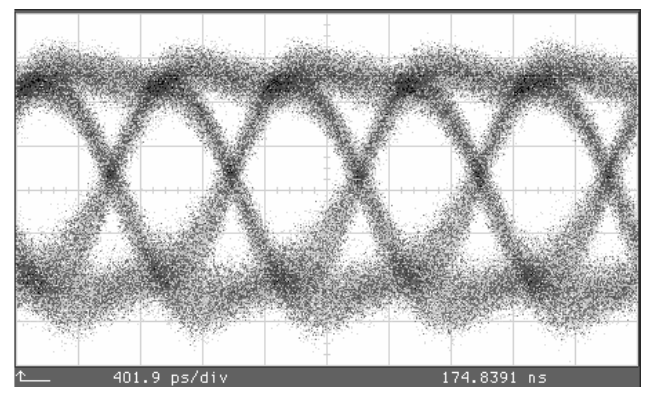

Figure 5: The electrical eye diagram at a receiver power of $-24 \mathrm{dBm}$.

\section{Conclusions}

A simplified novel photonic envelope detector for converting wireless signals into optical baseband ASK modulation based on a single Mach-Zehnder modulator and a fibre Bragg grating is presented. Envelope detection of $1.25 \mathrm{Gbit} / \mathrm{s}$ ASK modulated 35 $\mathrm{GHz}$ carrier was experimentally demonstrated, and a error free detection achieved.

\section{Acknowledgements}

This work has been carried out within EU FP6 IST IPHOBAC.

\section{References}

1. A. Hirata et al, IEEE MTT-S 54 (2006) 1937-1944

2. R.W. Ridgway et al, IEEE PTL 20 (2008), 653-655

3. R. Sambaraju et al, J. Lightw. Technol. 25 (2007) 3350-3357

4. R. Sambaraju et al, in Proc ECOC 2007, Tu5.4.7

5. K. Prince et al, Opt. Express 16 (2008) 2005-2013 



\section{Paper B}

Generation of Multi-Gigabit-per-Second MQAM/MPSKModulated Millimeter-Wave Carriers Employing Photonic Vector Modulator Techniques

R. Sambaraju, M. A. Piqueras, V. Polo, J. L. Corral, and J. Martí

Journal of Lightwave Technlogy, vol. 25, no. 11, pages 3350-3357, 2007. 


\title{
Generation of Multi-Gigabit-per-Second MQAM/MPSK-Modulated Millimeter-Wave Carriers Employing Photonic Vector Modulator Techniques
}

\author{
Rakesh Sambaraju, Student Member, IEEE, Student Member, OSA, Miguel Ángel Piqueras, Student Member, IEEE, \\ Valentín Polo, Member, IEEE, Juan L. Corral, Member, IEEE, and Javier Martí, Member, IEEE
}

\begin{abstract}
In this paper, two photonic vector modulator (PVM) architectures are presented, and their use in generating multigigabit-per-second M-ary quadrature amplitude modulation/ M-ary phase shift keying modulated RF carriers in the millimeter-wave frequency regime is experimentally demonstrated. First, a highly scalable photonic quadrature amplitude modulation (QAM) architecture based on vector summation and dispersive delay lines, which directly generate multilevel signals from parallel in-phase and quadrature components, is proposed and experimentally demonstrated by generating up to $3-\mathrm{Gb} / \mathrm{s}$ quadrature phase shift keying (QPSK), four-level amplitude shift keying, and eight-level QAM at 39-GHz-modulated carriers. The possibility of also detecting the baseband components is shown, which allows the simultaneous feeding of baseband/RF signals over the same infrastructure. This architecture is limited to a certain length of fiber, as the quadrature condition is obtained for a certain aggregated dispersion. To overcome this limitation, a second PVM architecture is proposed, which is based on the use of two Mach-Zehnder modulators in parallel and an optical delay line to obtain the quadrature condition. The generation of a 2-Gb/s QPSK signal is experimentally demonstrated, including a 1-km standard single-mode-fiber transmission.
\end{abstract}

Index Terms-Broadband access networks, frequency conversion, hybrid fiber-wireless access networks, microwave photonics, photonic vector modulators (PVMs), radio-on-fiber (RoF).

\section{INTRODUCTION}

B ROADBAND wireless access (BWA) networks are key last-mile-access technologies. BWA facilitates extending network coverage with relatively low deployment costs, faster revenue growth, and increased flexibility compared to common cabled infrastructures.

In parallel, wired local area networks and fiber-to-the-home access networks, which are experiencing a remarkable capacity increase, for instance $10 \mathrm{~Gb} / \mathrm{s}$ for gigabit Ethernet (GbE),

Manuscript received February 15, 2007; revised April 27, 2007. This work was supported by the European Commission FP6 under Project IST35317 IPHOBAC and Project IST-26592 ISIS and by the Spanish MCyT and EU-FEDER under Project TEC2005-08298-C02-02.

R. Sambaraju, V. Polo, and J. Martí are with the Nanophotonics Technology Center, Universidad Politécnica de Valencia, 46022 Valencia, Spain (e-mail: rsambaraju@ntc.upv.es; vpolor@ntc.upv.es; jmarti@ntc.upv.es).

M. Á. Piqueras is with DAS Photonics, S.L., Ciudad Politécnica de la Innovación, 46022 Valencia, Spain (e-mail: mapiqueras@ dasphotonics.com).

J. L. Corral was with the Departamento de Comunicaciones, Universidad Politécnica de Valencia, 46022 Valencia, Spain. He is now with the Nanophotonics Technology Center, Universidad Politécnica de Valencia, 46022 Valencia, Spain (e-mail: jlcorral@ntc.upv.es).

Digital Object Identifier 10.1109/JLT.2007.907795
$2.5 \mathrm{~Gb} / \mathrm{s}$ for gigabit passive optical network, and $3.2 \mathrm{~Gb} / \mathrm{s}$ for the IEEE 1394, are gaining momentum, as costs are decreasing due to higher market penetration, standardization, and use of low-cost optical technologies [1], [2].

This increasing capacity of wired data transmission has pushed the development of wireless technologies capable of transmitting high data rate signals, which is only possible by utilizing the millimeter-wave band. To reduce the utilized bandwidth, efficient modulation techniques such as quadrature amplitude modulation (QAM) or phase-shifted keying (PSK) modulations are employed. Typically, the generation of such modulation formats at microwave or millimeter-wave frequencies is electrically done by generating the modulation at an intermediate frequency and upconverting this signal up to the carrier frequency. However, when both the data rate and the carrier frequency are high, the modulation has to be directly generated at the carrier frequency, which requires devices with very stringent constraints in terms of bandwidth and linearity.

Radio-on-fiber (RoF) systems [3] have been proposed as enabling technologies to overcome the limitations of current electrical systems demonstrating multi-gigabit-per-second data rates wireless transmission at the millimeter-wave band [4]-[7]. However, the direct upconversion of multi-gigabit signals still imposes huge bandwidth requirements to the RF front end, as the upconverted signal occupies a passband bandwidth as huge as $20 \mathrm{GHz}$ centered at very high frequencies, which is typically $120 \mathrm{GHz}$ or above. At these frequencies, the signal suffers severe distance attenuation, which limits the maximum coverage area. Additionally, the hardware development at these frequencies is complicated and expensive.

As it happens in the case of electrical systems, the alternative is to generate the multilevel modulation formats employing photonic techniques [8]-[13]. Typically, they are based on the vector summation technique, which consists in achieving the desired carrier phase and amplitude by summing two orthogonal components, namely in-phase $(I)$ and quadrature $(Q)$ components.

In this paper, two photonic vector modulator (PVM) techniques are proposed and experimentally demonstrated. First, a highly scalable photonic QAM architecture based on vector summation and dispersive delay lines, which directly generate multilevel signals from parallel $I$ and $Q$ components, is proposed and experimentally demonstrated by generating up 


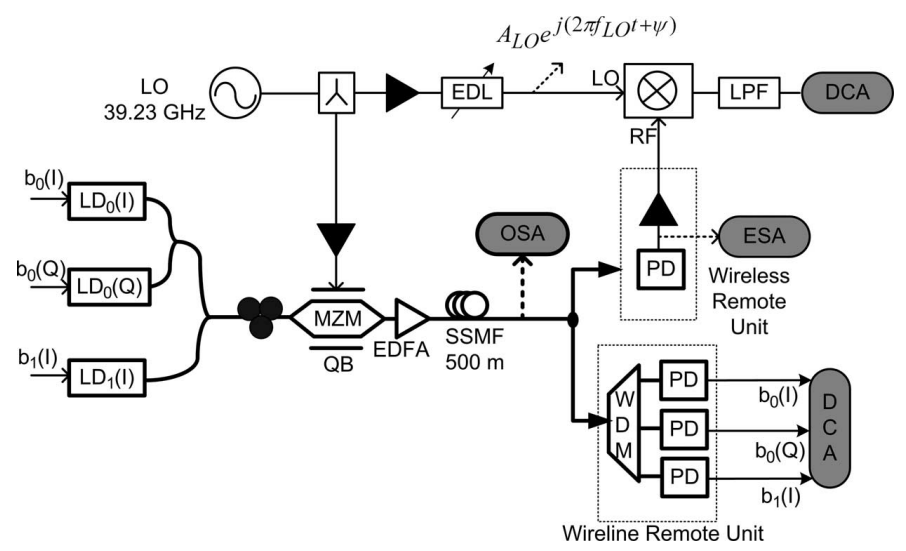

Fig. 1. Schematic of the proposed PVM architecture and phase-matched demodulation arrangement employed in the experiments. (OSA: optical spectrum analyzer. DCA: digital communications analyzer. ESA: electrical spectrum analyzer).

to 3-Gb/s quadrature phase shift keying (QPSK), four-level amplitude shift keying (4-ASK), and 8-QAM signals at $39 \mathrm{GHz}$, and confirming the capability of transmitting the original data streams at baseband, extending the results provided in [14]. The latter allows the simultaneous feeding of baseband/RF signals over the same infrastructure, as the generated signals can be detected both at baseband and upconverted to an arbitrary millimeter-wave frequency, in a highly flexible approach. At baseband, the digital data streams used to compose the multilevel signal can be directly detected after wavelength division demultiplexing the incoming wavelengths. At the same time, the digital data stream is upconverted and remodulated in a multilevel format directly over a RF carrier, which can be amplified, filtered, and radiated to a remote location over a wireless link. This architecture is limited to a certain length of fiber, as the quadrature condition is obtained for a certain aggregated dispersion.

To overcome this limitation, a second PVM architecture is proposed, which is based on the use of two Mach-Zehnder modulators (MZMs) in parallel and an optical delay line (ODL) to obtain the quadrature condition. The advantage of this architecture is that it allows fiber transmission over different spans, by controlling the transmission-induced delay between the two wavelengths using the ODL. The generation of a $2-\mathrm{Gb} / \mathrm{s}$ QPSK signal is experimentally demonstrated, including a 1-km standard single-mode-fiber (SSMF) transmission.

The organization of this paper is as follows. Section II describes the first PVM architecture, which is based on direct modulation of the lasers using baseband data, the RF carrier modulation using external modulator(s), and the quadrature condition using a dispersive element like a fiber. Experimental results are reported, and the application of this architecture is also discussed. Section III deals with the second proposed PVM architecture that is based on parallel MZMs and ODL, which solves the fiber-length-dependence constraint experienced by the architecture proposed in Section II. Again, the proposed PVM architecture is described, and the experimental results are reported. Finally, the conclusion is given in Section IV.

\section{PVM ARChitecture B ASED IN VECtor Summation}

\section{A. Architecture Description}

PVM architectures proposed in the literature are commonly based on two or more broadband electrooptical modulator arrangements, as well as complicated electrical control signals and electrical hybrids at the carrier frequency, which limits the useful bandwidth and increases the hardware complexity [4]-[7]. Fig. 1 depicts the PVM technique proposed in this paper, which employs a unique MZM, a dispersive medium as cheap as optical fiber, and baseband-modulated optical sources. This PVM architecture can be employed to implement superbroadband wireline/wireless heterogeneous access networks, as will be discussed in Section II-C.

The data source is a digital word formed by as many bits as required to obtain the desired modulation level. This parallel word has to be obtained from a digital bit stream, for instance, coming from a $\mathrm{GbE}$ router. The order of the generated modulation depends on the number of parallel bit streams. For example, to generate a 64QAM signal, a digital word of $\log _{2}(64)=6$ bits is required. This parallel digital stream directly modulates the lasers, which are standard optical sources widely employed, for instance in GbE systems. To generate the QAM signal, the even bits $b_{x}^{(I)}$ and lasers will form the in-phase component, and the odd bits $b_{x}^{(Q)}$ will form the quadrature one. The optical signals are combined by employing cascaded 3-dB optical coupler. In the MZM, all the directly modulated optical carriers are externally modulated by a local oscillator (LO) tone at the carrier frequency. Then, the optical signal is transmitted to the photodiode (PD) over a dispersive medium such as a standard single-mode optical fiber. Due to the chromatic dispersion of the fiber, a differential delay $\Delta \tau=D \cdot L \cdot \Delta \lambda$ among optical components is introduced [12], which depends on the wavelength spacing ( $\Delta \lambda$ [in nanometers]), the fiber length ( $L$ [in kilometers]), and the fiber dispersion parameter $(D[\mathrm{ps} / \mathrm{km} \cdot \mathrm{nm}])$. A $90^{\circ}$ phase shift is induced between the adjacent wavelengths by setting $\Delta \tau=T_{\mathrm{LO}} / 4$, where $T_{\mathrm{LO}}$ [in seconds] is the period of the LO signal. At the PD output, as 


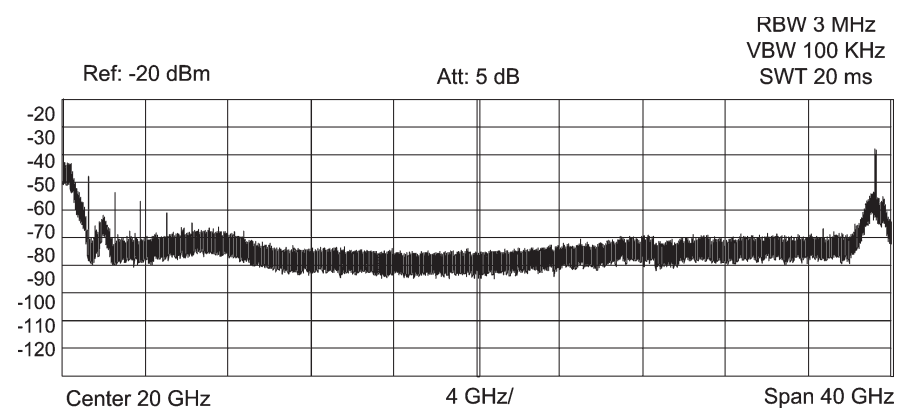

Fig. 2. Measured electrical spectrum at PD output. Both the baseband and multilevel RF components are clearly distinguished.

many replicas of the LO with differential phase shift of $90^{\circ}$ as bits are obtained. Employing the proposed architecture, it is possible to generate other modulation formats, such as QPSK or 4-ASK, by simply adjusting the phase relationship between the optical carriers by properly tuning the differential delay. The resulting normalized LO component of the photocurrent at the PD output yields

$$
\begin{aligned}
\frac{\overline{i_{\mathrm{LO}}}}{e^{j \omega_{\mathrm{LO}} t}}= & {\left[b_{0}^{(I)}-\frac{1}{2} b_{1}^{(I)}+\cdots+\frac{1}{(-2)^{N / 2}} b_{N / 2}^{(I)}\right] } \\
& +j\left[b_{0}^{(Q)}-\frac{1}{2} b_{1}^{(Q)}+\cdots+\frac{1}{(-2)^{N / 2}} b_{N / 2}^{(Q)}\right]
\end{aligned}
$$

This signal is a QAM at the LO frequency, except that the odd bits in both $I$ and $Q$ components have negative values. In fact, each QAM component is a multilevel signal with $2^{N / 2}$ linearly spaced levels, $N$ being the total number of bits.

With the proposed architecture, the QAM signal can be directly obtained from the parallel binary word, which results in a very simple and scalable architecture. In addition, since the multilevel signal is constructed at the PD output, no lasers with special linear modulation performance are required, simplifying the required hardware. An analysis on the tolerance of this PVM arrangement to drifts in the laser wavelength and LO frequency was reported in [12]. The system performance is not affected by the frequency chirp of the laser as the total link dispersion is controlled to maintain the $90^{\circ}$ relation between adjacent optical carriers, and the data signal bandwidth is small if compared to the optical carrier separation.

\section{B. Experimental Results}

The setup depicted in Fig. 1 was employed to validate the proposed approach. A set of three distributed feedback (DFB) lasers (1-GHz bandwidth), which is directly modulated by high-speed nonreturn-to-zero signals with $1-\mathrm{V}$ amplitude peak to peak, were employed to generate $2.5-\mathrm{Gb} / \mathrm{s} 4-\mathrm{ASK}$, 2.5-Gb/s QPSK, and 3-Gb/s 8-QAM signals at the LO frequency $(39.23 \mathrm{GHz})$. Only two lasers are employed to generate 4-ASK and QPSK signals, whereas all three are needed to generate the 8-QAM format. In all cases, the optical signals were polarization controlled and combined in a coupling matrix composed by two cascaded 3 -dB optical couplers. The optical carriers were centered at 1550.12, 1549.32, and $1548.52 \mathrm{~nm}$.
The corresponding optical signals were modulated by a $+15 \mathrm{dBm}$ LO in a $50-\mathrm{GHz}$ MZM biased at the quadrature point, amplified by an Erbium-doped fiber amplifier (EDFA), transmitted over $0.5 \mathrm{~km}$ of single-mode fiber (SMF) $(D=$ $16.7 \mathrm{ps} / \mathrm{km} \cdot \mathrm{nm}$ ), and photodetected. The optical power at the input of the PD was $+2.5 \mathrm{dBm}$.

In Fig. 2, the electrical spectrum of the generated signals, which is measured with an electrical spectrum analyzer (ESA), is depicted, where both the baseband data and the upconverted signal at $39.23 \mathrm{GHz}$ can be clearly distinguished. Only the spectrum corresponding to the case of generating a $2.5-\mathrm{Gb} / \mathrm{s}$ 4-ASK signal is shown, as it is analogous to the spectrum obtained for the QPSK and 8-QAM cases. As shown in Fig. 1, in all cases, the multilevel RF carrier was demodulated by mixing a phase-adjusted replica of the LO with the signal, and eye diagrams were measured using a digital communications analyzer (DCA).

Figs. 3 and 4 show the results of the 2.5-Gb/s downconverted signal for the cases of 4-ASK modulation and QPSK modulation, respectively. In these cases, the two lasers were directly modulated by a $1.25-\mathrm{Gb} / \mathrm{s}$ bit stream. It should be noticed that, in the case of generating a 4-ASK signal, an envelop detector can be used at the receiver side in a real implementation, avoiding the need of wideband electrical mixers and phaselocked loops [4], resulting in a very simplified and low-cost solution.

Finally, the three lasers were switched on to generate a 3-Gb/s 8-QAM signal at 39.23 GHz. Each laser was modulated with a $1-\mathrm{Gb} / \mathrm{s}$ bit stream. Fig. 5 shows the demodulated eye diagrams of both $I$ and $Q$ components, as well as an intermediate eye diagram. The four levels of the $I$ component and the two levels of the $Q$ component of the generated 8-QAM signal can be clearly distinguished.

\section{Simultaneous Delivery of Gigabit-per-Second Date at Baseband and RF for Wireline/Wireless Convergence}

Wireline and wireless infrastructure coexistence will continue for the next decade, as neither wireline nor BWA networks offer disruptive advantages in all scenarios. RoF techniques in this respect offer a highly flexible approach to multiservice hybrid access networks. RoF techniques fostering infrastructure sharing, multistandard capabilities, and wireline and wireless access coexistence have been therefore intensively 


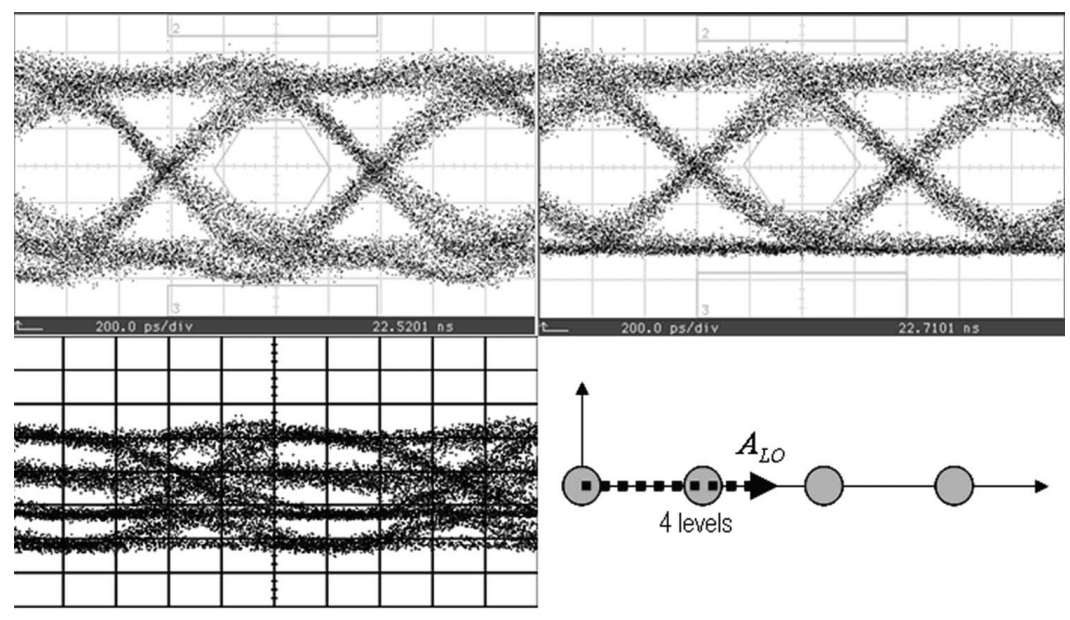

Fig. 3. Results for 4-ASK generation. (Top) Demodulated eye diagrams of the (left) $b_{0}^{(I)}$ and (right) $b_{1}^{(I)}$ data streams. (Bottom left) Demodulated eye and (right) generated 4-ASK constellation diagram.

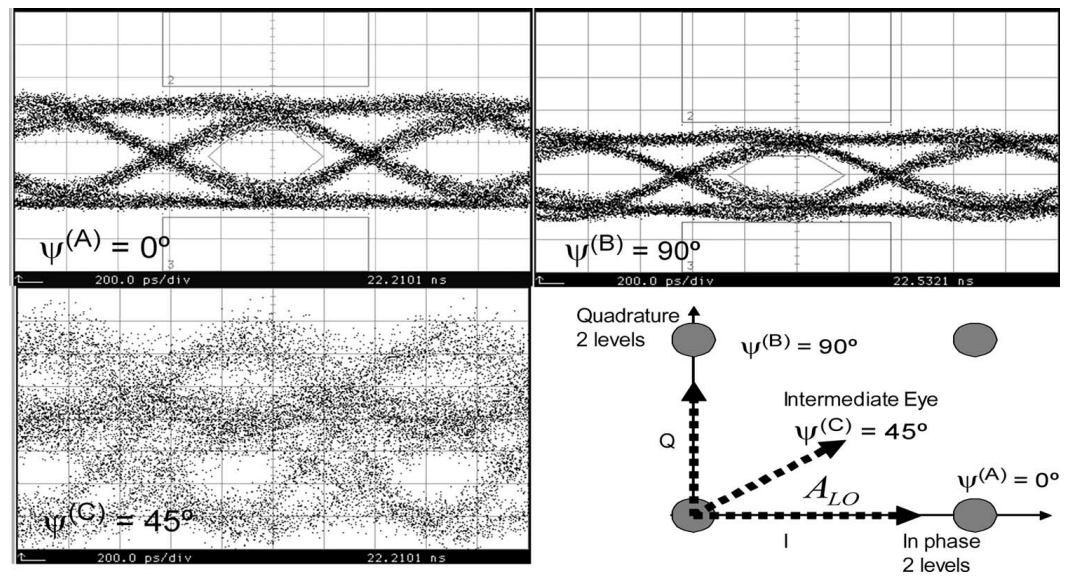

Fig. 4. Results for QPSK generation. (Top) Demodulated eye diagrams of the (left) $I$ and (right) $Q$ components (the GbE receiving mask is shown, compared with the received eye diagrams). (Bottom left) Intermediate eye and (right) generated QPSK constellation diagram.
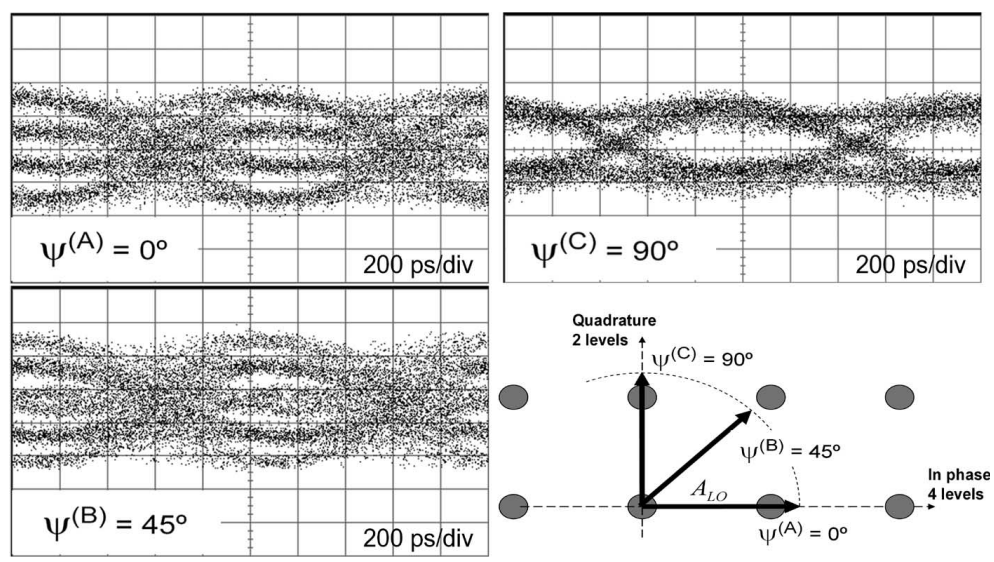

Fig. 5. Results for 8-QAM generation. (Top) Demodulated eye diagrams of the (left) $I$ and (right) $Q$ components. (Bottom left) Intermediate eye and (right) generated 8-QAM constellation diagram. 


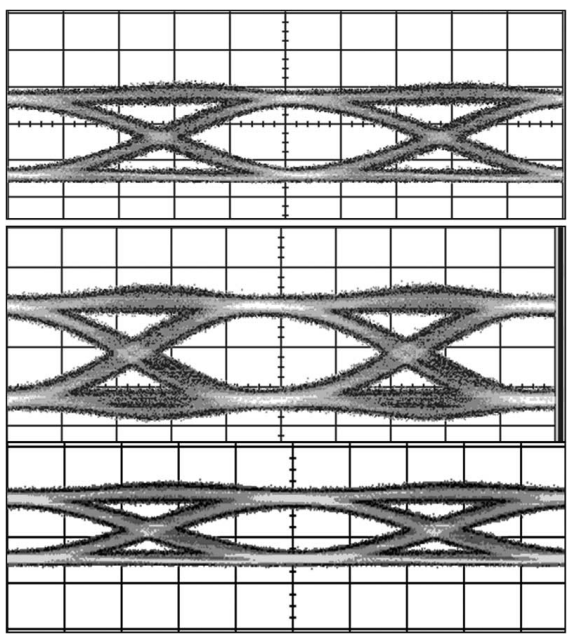

Fig. 6. Measured eye diagrams of $1-\mathrm{Gb} / \mathrm{s}$ parallel bit streams after demultiplexing measured by the DCA. (Top) $b_{0}^{(I)}$ at $1550.12 \mathrm{~nm}$, (middle) $b_{0}^{(Q)}$ at $1549.32 \mathrm{~nm}$, and (bottom) $b_{1}^{(I)}$ at $1548.52 \mathrm{~nm}$. Horizontal scale is $200 \mathrm{ps}$, and vertical scale is $20 \mathrm{mV}$.

investigated and demonstrated, due to their potential impact in reducing capital expenditure and operational expenditure costs [14]-[16].

The proposed PVM architecture can be an alternative solution in this framework, as it is possible to simultaneously obtain the baseband data used to generate the multilevel signal, and the upconverted multilevel modulated RF carrier. To demonstrate this feature, the setup shown in Fig. 1 was used to generate a 3-Gb/s 8-QAM signal. As shown in Fig. 1, the three wavelengths are obtained by demultiplexing the optical signal prior to photodetection, and then, it is possible to obtain the parallel bit streams used to simultaneously generate the multilevel signal $b_{x}(I)$ and the corresponding multilevel modulation at the RF carrier frequency.

The measured eye diagrams of each parallel bit stream are shown in Fig. 6. $Q$ parameters of $11.9,11.8$, and 9.5 were estimated by the DCA software, respectively. These bit streams can be serialized to get the original 3-Gb/s bit stream or directly fed to an optical switch that can handle the incoming parallel data streams directly in the optical domain.

The proposed PVM architecture is a highly flexible approach to simultaneously provide broadband data at baseband frequencies and at RF. This architecture is limited to a certain length of fiber, as the quadrature condition is obtained for a certain aggregated dispersion. If dispersion management is included, to cope with differences in fiber spans within the network, the proposed arrangement will find application in real scenarios, as proposed in [18].

\section{PVM ARChiteCtURE BASED IN TANDEM MZMS AND ODLS}

To overcome the fiber-length dependence of the quadrature condition, several approaches can be used, for instance, using

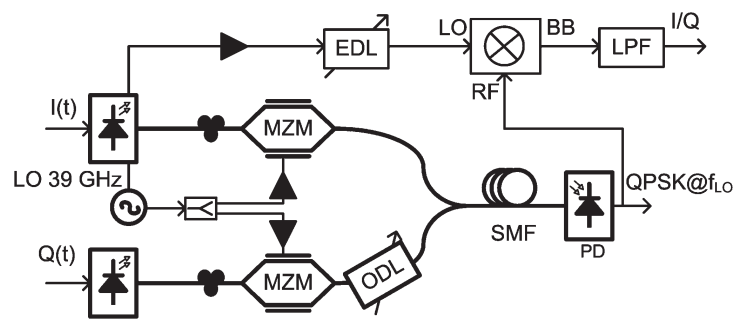

Fig. 7. Schematic of the proposed QPSK modulator and the experimental arrangement used to demodulate the generated QPSK millimeter-wave carrier.

tunable lasers, ODLs, or dispersion-compensating modules. In this paper, a second PVM architecture is proposed, based on the use of two MZMs in parallel, and an ODL to obtain the quadrature condition.

\section{A. Architecture Description}

As shown in Fig. 7, the generation of a QPSK-modulated carrier is performed in three steps. First, the two DFB lasers are modulated by the $I$ and $Q$ bit streams. In this case, the lasers are also standard components for digital baseband applications. Second, a millimeter-wave LO tone externally modulates the incoming optical carriers in a MZM biased at its quadrature-bias point. Finally, the quadrature condition is obtained by delaying the output of the MZM of the $Q$ arm using a tunable ODL.

The LO current component at the output of the PD can be calculated as

$$
\begin{aligned}
i_{\mathrm{PD}}(t)= & \frac{\Re}{t_{\mathrm{ff}} L_{\mathrm{OPT}}} J_{0}(m) J_{1}(m) \\
& \times\left[(P+\eta I(t)) \cdot \cos \left(\omega_{\mathrm{LO}} t\right)+(P+\eta Q(t))\right. \\
& \left.\quad \cdot \cos \left(\omega_{\mathrm{LO}}\left(t-T_{\mathrm{ODL}}\right)+\beta_{2} L \omega_{\mathrm{LO}} \Delta \omega\right)\right]
\end{aligned}
$$

where $t_{\mathrm{ff}}$ is the MZM coupling losses, $L_{\mathrm{OPT}}$ are the losses associated to the optical fiber attenuations, $P$ is the mean output power of the laser, $\eta$ is the internal efficiency of laser, $I(t)$ and $Q(t)$ are the in-phase and quadrature baseband data currents, $\Delta \lambda$ is the wavelength separation, and $\Delta \omega$ is the frequency separation of lasers. $J_{n}()$ is the $n$ th-order Bessel function of the first kind, $T_{\mathrm{ODL}}$ is the delay induced by the ODL, $\beta_{2}$ is the group velocity dispersion parameter, and $m=\pi V_{\mathrm{LO}} / V \pi$ is the LO modulation index, $V_{\mathrm{LO}}$ being the amplitude of the LO component at the input of each MZM, and $V_{\pi}$ being the half-wave voltage of the modulator.

The optical delay required to obtain the quadrature condition, as shown in the following equations, is expressed as (3) when no fiber transmission is considered and as (4) when the optical field travels through a fiber span:

$$
\begin{array}{ll}
T_{\mathrm{ODL}}=\frac{(2 k+1)}{4 f_{\mathrm{LO}}}, & k=1,2, \ldots \\
T_{\mathrm{ODL}}=\mathrm{DL} \Delta \lambda-\frac{(2 k+1)}{4 f_{\mathrm{LO}}}, & k=1,2, \ldots
\end{array}
$$

The difference between this architecture with respect to the one proposed in Section II is that, in the first case, the delay 


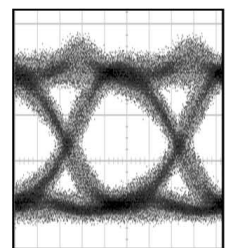

(a)

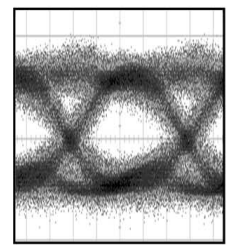

(c)

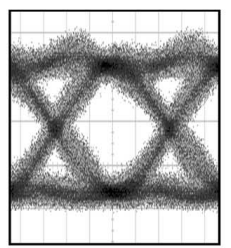

(b)

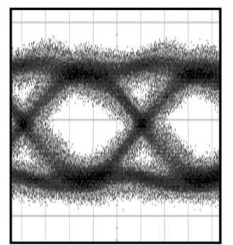

(d)

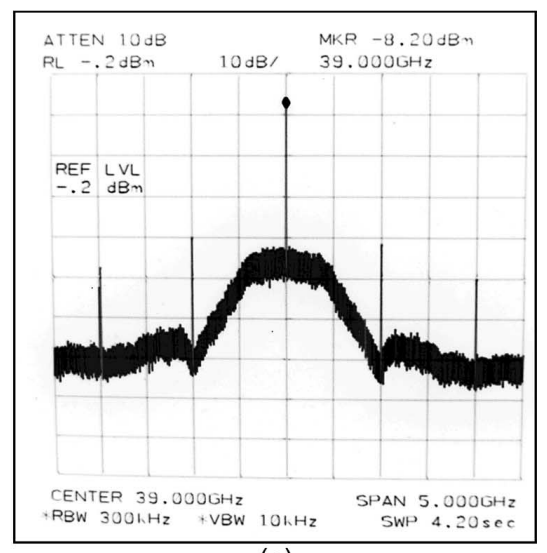

(e)

Fig. 8. Demodulated baseband eye diagrams. (Top) Back-to-back (a) $I$ and (b) $Q$ components and (bottom), after 1-km fiber transmission, (c) $I$ and (d) $Q$ components. Scale X: $200 \mathrm{ps} / \mathrm{div}$, Y: $10 \mathrm{mv} /$ div. (e) RF spectrum. Scale: X $10 \mathrm{~dB} / \mathrm{div}, \mathrm{Y}$ : $0.5 \mathrm{GHz} / \mathrm{div}$.

required for obtaining the $90^{\circ}$ condition in the LO envelop is obtained by dispersion-induced differential delay [12], and in the second one, it is obtained by propagation differential delay introduced with an ODL placed in one of the modulator branches. It is worth stating that, in this second case, when a fiber span is included in the system, which is the normal situation in all the applications, the dispersion-induced delay can be dynamically compensated with the ODL, maintaining the quadrature condition $\left(90^{\circ}\right.$ phase shift) for any fiber length. This is the case shown in (4). There would be certain fiberlength values whose dispersion would induce the suppression of the detected RF carrier [19]. These fiber lengths should be avoided or any conventional dispersion compensation techniques or dispersion-tolerant modulation as optical single sideband should be used. This situation would never occur in the technique shown in Section II, where the link dispersion would always be too low to induce the carrier suppression effect.

\section{B. Experimental Results}

As a proof of concept, the generation and fiber transmission of a 2-Gb/s QPSK signal generated at $39 \mathrm{GHz}$ was performed. Two directly modulated DFB lasers (1-GHz bandwidth) at wavelengths 1550 and $1548.211 \mathrm{~nm}$ were used. Two pseudorandom sequences $I(t)$ and $Q(t)$ at a baseband data rate of $1 \mathrm{~Gb} / \mathrm{s}$ were used to modulate the 1548.211 - and $1550-\mathrm{nm}$ lasers, respectively. The electrical voltage (peak to peak) of the baseband data $I(t)$ was set to $2.4 \mathrm{~V}$, and $3.3 \mathrm{~V}$ for $Q(t)$, resulting in optical powers of 6.53 and $7.71 \mathrm{dBm}$, respectively. To generate ideal QPSK signals, the optical power of the two arms $(I$ and $Q$ ) should be nearly equal, and to achieve this, the driving voltages were differently chosen to compensate the 2-dB loss of the ODL present in the $Q$ arm. Two 40-GHz MZM biased at the quadrature point were used to modulate the LO signal. The LO was split into three arms, where two arms were amplified to $+15 \mathrm{dBm}$ power to be fed to the optical modulator, and the third arm to the QAM demodulator arrangement. The output of the $3-\mathrm{dB}$ coupler was photodetected using a $40-\mathrm{GHz}$ photoreceiver. To analyze the generated electrical QPSK signal, it was coherently demodulated using the same LO used in the transmitter, employing an electrical delay line (EDL) to synchronize the LO carrier. The output of the mixer is lowpass filtered using a baseband filter, and the output is monitored on a DCA. Looking at the eye diagram, the optical delay was adjusted to exactly tune to the quadrature condition.

The demodulated baseband signals $Q$ factor was estimated using the DCA built-in software routines. In the back-to-back case, measured $Q$ factors were 9.1 and $9.2 \mathrm{~dB}$ for the detected $I$ and $Q$ components. Later, the optical signal was transmitted over a 1-km SMF. Due to the fiber-dispersion-induced delay, the quadrature condition was lost but was regained by retuning the ODL to 24 ps. In this case, the demodulated signal's $Q$ factor was estimated to be 5.9 and $6.26 \mathrm{~dB}$ for $I$ and $Q$ components, respectively. The transmission $Q$-factor penalty is around $3 \mathrm{~dB}$. This penalty can be reduced by clipping the zero levels of the optical baseband data but at the cost of reduced optical power. Fig. 8 shows the demodulated eye diagrams of the system in the back-to-back case (top) and after 1-km fiber transmission (bottom).

The advantage of the proposed system is its independence of wavelength separation and the length of fiber transmission compared to the previously proposed architecture at the cost of increased hardware.

\section{Conclusion}

In this paper, two PVM architectures based on the vector summation technique and fiber-dispersion-induced delays have been proposed.

The first PVM architecture, the generation of multilevel modulated carriers directly at the carrier frequency from parallel binary streams is achieved. The required hardware and the modulator control are simpler and scalable than the previously proposed architectures. The proposed architecture has been experimentally evaluated by generating $2.5-\mathrm{Gb} / \mathrm{s}$ 4-ASK, 
2.5-Gb/s QPSK, and 3-Gb/s 8-QAM at 39-GHz carriers. This scheme is suitable for remote antenna applications by using dispersion management in the fiber span, and the lasers, coupling matrix, and the modulator could be integrated in a single photonic chip. Moreover, the feasibility of simultaneously deploying multi-gigabit-per-second signals at baseband and millimeter-wave frequencies has been demonstrated in a highly flexible approach.

A second PVM architecture for generating QPSK signals at LO frequency using direct modulation of baseband data, two MZM in tandem, and an ODL has been proposed. By detuning the ODL, it is possible to independently achieve the quadrature condition of the fiber length. As a proof of concept, the generation and 1-km fiber transmission of a 2-Gb/s QPSK modulated at 39-GHz RF carrier has been demonstrated.

PVM techniques are highly attractive as they can overcome the limitation of electrical systems to generate multi-gigabitper-second multilevel signals in the millimeter-wave band. Moreover, the possibility to develop integrated PVM architectures employing photonic integration technologies makes them very attractive in terms of size and power consumption. Finally, the possibility of simultaneously delivering the baseband components and the upconverted multilevel modulated RF carrier to the access node allows fostering wireline/wireless infrastructure convergence, which is a key feature for the successful deployment of truly broadband heterogeneous access networks.

\section{REFERENCES}

[1] IEEE Std. 802.3TM-2002. IEEE Computer Society. [Online]. Available: http://www.ieee802.org/3/hssg/index.html

[2] F.-T. An, D. Gutierrez, K. K. Soo, L. J. Woo, and G. Kazovsky, "SUCCESS-HPON: A next-generation optical access architecture for smooth migration from TDM-PON to WDM-PON," IEEE Commun. Mag., vol. 43, no. 11, pp. S40-S47, Nov. 2005.

[3] A. J. Seeds and J. K. Williams, "Microwave photonics," J. Lightw. Technol., vol. 24, no. 12, pp. 4628-4641, Dec. 2006.

[4] M. Á. Piqueras, B. Vidal, H. Pfrommer, V. Polo, A. Ramírez, and J. Martí, "Radio-over-fiber multi-service MM-wave interconnection with photonic up-conversion, dual band remote delivery and photonic envelop detection," in Proc. IEEE MTT-S, Intl. Microwave Symp., San Francisco, CA, Jun. 2006, pp. 2035-2038.

[5] A. Hirata, M. Harada, and T. Nagatsuma, "120-GHz wireless link using photonic techniques for generation, modulation, and emission of millimeter-wave signals," J. Lightw. Technol., vol. 21, no. 10, pp. 21452153, Oct. 2003.

[6] K. Ohata, K. Maruhasi, M. Ito, S. Kishimoto, K. Ikuina, T. Hashiguchi, K. Ikeda, and N. Takahashi, "1.25 Gbps wireless gigabit Ethernet link at 60 GHz-band,” in Proc. IEEE Int. Microw. Symp., 2003, pp. 373-376.

[7] T. Nagatsuma, A. Hirata, M. Harada, H. Ishii, K. Machida, T. Minotani, H. Ito, T. Kosugi, and T. Shibata, "Millimeter-wave photonic integrated circuit technologies for high-speed wireless communications applications," in Proc. IEEE ISSCC, 2004, vol. 1, pp. 448-449.

[8] P. Candelas, J. M. Fuster, J. Martí, and J. C. Roig, "Optically generated electrical-modulation formats in digital-microwave link applications," J. Lightw. Technol., vol. 21, no. 2, pp. 496-499, Feb. 2003.

[9] A. Wiberg, P. Perez-Milan, M. V. Andres, P. A. Andrekson, and P. O. Hedekvist, "Fiber-optic $40-\mathrm{GHz} \mathrm{mm}$-wave link with $2.5-\mathrm{Gb} / \mathrm{s}$ data transmission," IEEE Photon. Technol. Lett., vol. 17, no. 9, pp. 1938-1940, Sep. 2005.

[10] J. F. Coward, C. H. Chalfant, and P. H. Chang, "A photonic integratedoptic RF phase shifter for phased array antenna beam-forming applications," J. Lightw. Technol., vol. 11, no. 12, pp. 2201-2205, Dec. 1993.

[11] W. D. Jemison, A. J. Kreuzberger, and E. Funk, "Microwave photonic vector modulator for high-speed wireless digital communications," IEEE Microw. Wireless Compon. Lett., vol. 12, no. 4, pp. 125-127, Apr. 2002.

[12] M. Á. Piqueras, B. Vidal, V. Polo, J. L. Corral, and J. Martí, "Direct photonic generation of electrical vector modulations at microwave/ millimeter-wave frequencies," IEEE Photon. Technol. Lett., vol. 17, no. 9, pp. 1947-1949, Sep. 2005

[13] M. Á. Piqueras, B. Vidal, J. L. Corral, V. Polo, H. Pfrommer, A. Martínez, and J. Martí, "Photonic vector modulation Tx/Rx architecture for generation, remote delivery and detection of M-QAM signals," in Proc. IEEE MTT-S Int. Microw. Symp., Long Beach, CA, Jun. 12-17, 2005, pp. 27-30.

[14] M. Á. Piqueras, V. Polo, J. L. Corral, B. Vidal, A. Martínez, and J. Martí, "Radio-over-fibre broadband quadrature amplitude modulator," in Proc. ECOC, Cannes, France, 2006, vol. 2, p. 43.

[15] C. Lim, A. Nirmalathas, D. Novak, R. Waterhouse, and G. Yoffe, "Millimeter-wave broad-band fiber-wireless system incorporating baseband data transmission over fiber and remote LO delivery," J. Lightw. Technol., vol. 18, no. 10, pp. 1355-1363, Oct. 2000.

[16] K. Ikeda, T. Kuri, and K. Kitayama, "Simultaneous three-band modulation and fiber-optic transmission of $2.5-\mathrm{Gb} / \mathrm{s}$ baseband, microwave-, and 60-GHz-band signals on a single wavelength," J. Lightw. Technol., vol. 21, no. 12 , pp. 3194-3202, Dec. 2003.

[17] C. P. Liu, V. Polo, F. Van Dijk, H. Pfrommer, M. Á. Piqueras, J. Herrera, A. Martinez, S. Karlsson, O. Kjebon, R. Schatz, A. Enard, Y. Yu, T. Tsegaye, C. H. Chuang, A. J. Seeds, and J. Martí, "Full-duplex DOCSIS/wireless DOCSIS fiber-radio network employing packaged AFPMs as optical/electrical transducers," J. Lightw. Technol., vol. 25, no. 3, pp. 673-684, Mar. 2007.

[18] M. Á. Piqueras, V. Polo, and J. Martí, "Simultaneous base-band and mm-wave delivery of Gbps data employing photonic vector modulators," presented at the Optical Fiber Commun. Conf. (OFC), Anaheim, CA, 2007, Paper JThA60.

[19] G. J. Meslener, "Chromatic dispersion induced distortion of modulated monochromatic light employing direct detection," IEEE J. Quantum Electron., vol. QE-20, no. 10, pp. 1208-1216, Oct. 1984.

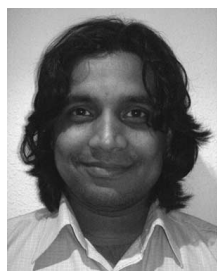

Rakesh Sambaraju (S'07) was born in Shadnagar, India, in 1980. He received the B.Tech. degree in electronics and communications engineering from Jawaharlal Nehru Technical University, Hyderabad, India, in 2002 and the M.Sc. degree in telecommunications from the Technical University of Denmark, Lyngby, Denmark, in 2006. He is currently working toward the Ph.D. degree at the Universidad Politécnica de Valencia (UPV), Valencia, Spain.

$\mathrm{He}$ is with the Nanophotonics Technology Center, UPV, where he is currently involved with the FP6 project IPHOBAC and national project GODIR. His research interests include optical modulation formats, external modulators, photonic vector modulation, and radio-over-fiber systems.

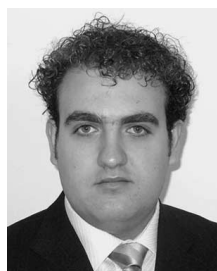

Miguel Ángel Piqueras (S'01) was born in Tarazona de la Mancha, Spain, on October 20, 1978 He received the Ingeniero de Telecomunicación degree from the Universidad Politécnica de Valencia Valencia, Spain, in 2003, where he is currently working toward the $\mathrm{Ph} . \mathrm{D}$. degree.

For four years, he was with the Nanophotonics Technology Center (NTC), Universidad Politécnica de Valencia, as a Researcher in optical beamforming networks, photonic vector modulation architectures, and microwave and millimeter-wave optical fibe systems. He is currently with DAS Photonics, S.L., Ciudad Politécnica de la Innovación, Valencia (an NTC spin-off) as a Project Manager, working on siliconphotonics and radio-over-fiber solutions for space and defense applications. He has participated in five European and four National Research Projects. He has published more than 40 papers in international journals and conferences, as well as three contributions in standardization bodies. 


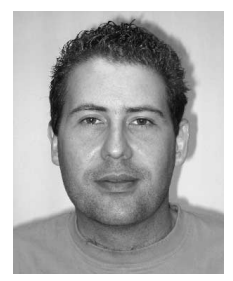

Valentín Polo (S'92-A'03-M'04) received the Ingeniero de Telecomunicación and Ph.D. degrees from the Universidad Politécnica de Valencia (UPV), Valencia, Spain, in 1997 and 2003, respectively. His Ph.D. dissertation on "Applications of ElectroAbsorption Modulators in Radio-over-Fibre Systems" received the Cátedra Telefónica-UPV 2005 Award.

He is with the Nanophotonics Technology Center, UPV, where he is involved with research and technical management levels in national and European R\&D projects dealing with microwave-photonics and broadband access networks. The main projects in which he has participated are FP5 IST-2000 25390 "OBANET" and FP6 IST-1-507781 GANDALF (as a Technical Manager). He is currently involved with the PF6 projects IPHOBAC and ISIS. He is a coauthor of more than 90 papers in international journals and conference proceedings. His research interests include external modulators, photonic-microwave filters, dispersion compensation, and broadband wireline and wireless access technologies and systems.

Dr. Polo is a Reviewer for several journals and conferences of the IEEE, IEE, the Optical Society of America, and the European Union Information Society Technologies.

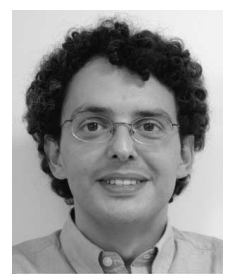

Juan L. Corral (''91-A'99-M'01) was born in Zaragoza, Spain, on April 20, 1969. He received the Ingeniero de Telecomunicación degree (with firstclass honors) and the Doctor Ingeniero de Telecomunicación degree from the Universidad Politécnica de Valencia, Valencia, Spain, in 1993 and 1998, respectively. He was a recipient of the 1998 Doctorate Prize of the Telecommunications Engineer Association in Spain for his Ph.D. dissertation on applications of MMIC and photonic technologies to phased array antennas.

In 1993, he was an Assistant Lecturer with the Departamento de Comunicaciones, Universidad Politécnica de Valencia. From 1993 to 1995, he was with the Microwave Technology and Equipment Section (XRM), European Space Research and Technology Center, European Space Agency, the Netherlands, where he was engaged in research on MMIC-based technologies and photonics technologies for beamforming networks for on-board phased array antennas. In 1995, he joined the Departamento de Comunicaciones, Universidad Politécnica de Valencia, where he became an Associate Professor in 2000. He is currently with the Nanophotonics Technology Center, Universidad Politécnica de Valencia. He is the author or coauthor of more than 70 papers in international journal and conference proceedings. His research interest include phased array antennas, optical beamforming networks, and microwave and millimeter-wave optical fiber systems.

Dr. Corral is a member of several IEEE societies.

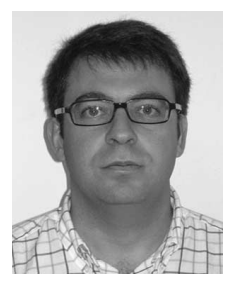

Javier Martí (S'89-M'92) received the Ingeniero de Telecomunicación degree from the Universidad Politécnica de Catalunya, Barcelona, Spain, in 1991 and the Doctor Ingeniero de Telecomunicación degree (Ph.D.) from the Universidad Politécnica de Valencia, Valencia, Spain, in 1994.

From 1989 to 1990 , he was an Assistant Lecturer with the Escuela Universitaria de Vilanova, Barcelona. From 1991 to 2000, he was a Lecturer and an Associate Professor with the Telecommunication Engineering Faculty, Universidad Politécnica de Valencia, where he is currently a Full Professor. In 2003, he was appointed as the Director of the Nanophotonics Technology Center, Universidad Politécnica de Valencia. He is the author or coauthor of more than 200 papers in referred international technical journals. He is the holder of 12 patents in broadband hybrid fiber-radio systems and microwave/millimeter-wave photonics, fiberbased access networks, terabit per second OTDM/WDM optical networks, advanced optical processing techniques for microwave signals and ultrahigh speed data transmission, and (silicon) integrated photonics, including planar photonic crystals and metamaterials. He has very actively participated in the European Research Framework (FP5 and FP6), leading several projects in broadband radio-over-fiber access (OBANET, GANDALF), all-optical processing in photonic networks (LASAGNE), and nanophotonic logic gates (PHOLOGIC).

Prof. Martí was a member of Technical Program Committee of the European Conference on Optical Communication, the IEEE Lasers and Electro-Optics Society, the IEEE Microwave Photonics, and other international workshops and conferences. 



\title{
Paper C
}

Performance Analysis of Photonic Vector Modulation Techniques for Multi-Gb/s Wireless Links

\author{
R. Sambaraju, J. L. Corral, J. Palaci, V. Polo, and J. Martí
}

Journal of Lightwave Technlogy, vol. 26, no. 15, pages 2684-2691, 2007. 


\title{
Performance Analysis of Photonic Vector Modulation Techniques for Multi-Gb/s Wireless Links
}

\author{
Rakesh Sambaraju, Student Member, IEEE, Student Member, OSA, Juan Luis Corral, Member, IEEE, \\ Jesús Palací, Student Member, IEEE, Valentín Polo, Member, IEEE, and Javier Martí, Member, IEEE
}

\begin{abstract}
Broadband access networks evolution towards 10-Gb/s user connectivity will foster the evolution of the photonic and wireless technologies needed to implement multi-Gb/s wireless links. In particular, provision of data rates in excess of $1 \mathrm{~Gb} / \mathrm{s} \mathrm{using}$ wireless technologies is limited by currently available electrical technologies due to the required bandwidths and frequencies of operation in the millimeter-wave (mm-wave) band. Microwave photonic techniques and technologies have shown a clear potential to overcome these limitations. In this paper, the detailed study of a photonic vector modulator (PVM) architecture and its performance limitations are presented. The PVM architecture is based on direct baseband modulation and dispersion induced quadrature condition. The limitation of this architecture is the presence of a local oscillator (LO) component in the generated $\mathrm{mm}$-wave spectrum which limits the system dynamic range. To overcome this limitation, a third continuous-wave (CW) laser is used to remove the unwanted $\mathrm{LO}$ component by properly adjusting its wavelength and emitted power. After presenting the theoretical model, the LO suppression feature is demonstrated experimentally by generating a 1.25-Gb/s QPSK 41-GHz modulated carrier. The two options (with and without LO carrier) are compared in terms of relevant performance parameters.
\end{abstract}

Index Terms-Frequency conversion, hybrid fiber-wireless access networks, microwave photonics, photonic vector modulators, radio-on-fiber.

\section{INTRODUCTION}

B ROADBAND access networks are evolving towards $10-\mathrm{G}$ connectivity. The possibility of providing such data rates wirelessly is highly interesting due to the faster and cheaper deployment, quicker revenue, user ubiquity, or reconfiguration/flexibility, features inherent to broadband wireless access networks. In addition, redundancy/back-up solutions to improve network performance under disaster or service disruption situations can be offered by providing a converged network infrastructure at PHY level between wireline and wireless links [1]-[4]. With the widespread adoption of fiber-to-the-home (FTTH) access networks, wireless transmission of multi-Gb/s

Manuscript received January 30, 2008; revised March 26, 2008. Current version published October 10, 2008. This work has been supported by the European Commission FP6 under projects IST-35317 IPHOBAC. Financial support by the Spanish MCyT and EU-FEDER under the project TEC2005-08298-C02-02 and the Valencia Regional Government under the project ACOMP2007/144 is gratefully acknowledged.

The authors are with the Valencia Nanophotonics Technology Center, Universidad Politécnica de Valencia, 46022 Valencia, Spain (e-mail: rsambaraju@ntc.upv.es; jlcorral@ntc.upv.es; jespalpe@ntc.upv.es; vpolor@ntc. upv.es; jmarti@ntc.upv.es).

Color versions of one or more of the figures in this paper are available online at http://ieeexplore.ieee.org.

Digital Object Identifier 10.1109/JLT.2008.925659 signals has raised a lot of research interest in order to provide a solution to implement the wireless extension of FTTH networks, also known as fiber-to-the-air (FTTA). Radio-over-fiber technologies [5] offer the required bandwidth and flexibility to implement these challenging communication systems, overcoming the main limitations of purely electrical approaches [2], [6], [7]. However, conventional methods based on direct upconversion of the digital baseband signal [6]-[8] suffer from huge bandwidth constraints, in particular when thinking on realistic full-duplex deployments where the used bandwidth is required in both downstream and upstream links. Therefore, the use of more spectrally efficient modulation formats such as multilevel quadrature amplitude or phase modulation (MQAM or MPSK, respectively) is of high interest to alleviate such problems [9], [10]. Recently, several photonic vector modulator (PVM) architectures with low hardware requirements have been proposed, both optical and electrical, showing the potential of this approach to generate up to $3.6-\mathrm{Gb} / \mathrm{s} 16$ QAM at $40-\mathrm{GHz}$ carriers [11]-[13].

In this paper, the detailed modeling of the PVM architecture proposed in [11] is presented. This PVM architecture is based on direct modulation and dispersion induced quadrature condition. The limitation of this architecture is the presence of a local oscillator (LO) component in the generated millimeterwave (mm-wave) spectrum which limits the system dynamic range [12], [13]. To overcome this limitation, a third CW laser is used to remove the unwanted LO component, by properly adjusting its wavelength and emitted power [14]. The detailed theoretical model of both PVM generation options (with and without LO carrier) is presented in this paper, and their performances are compared in terms of relevant operating parameters. After presenting the theoretical model, the LO suppression feature is demonstrated experimentally by generating a $1.25-\mathrm{Gb} / \mathrm{s}$ QPSK 41-GHz modulated carrier.

The paper is organized as follows. In Section II, the PVM architecture and its modification to remove the LO component is described. In Section III, the corresponding mathematical models are provided, deriving the photocurrent and noise contributions of the PVM architectures required to obtain the signal-to-noise-ratio (SNR). The optimum operating points of the PVM system are obtained from the equations. In Section IV, the experimental results demonstrating the LO component suppression using a third laser are reported. A 1.25-Gb/s QPSK 41-GHz carrier with and without LO component are generated using the proposed approach. Next, performance limitations of the proposed PVM architecture are discussed after the experimental comparison between the original PVM architecture with LO component [11] and without the LO component [14] 

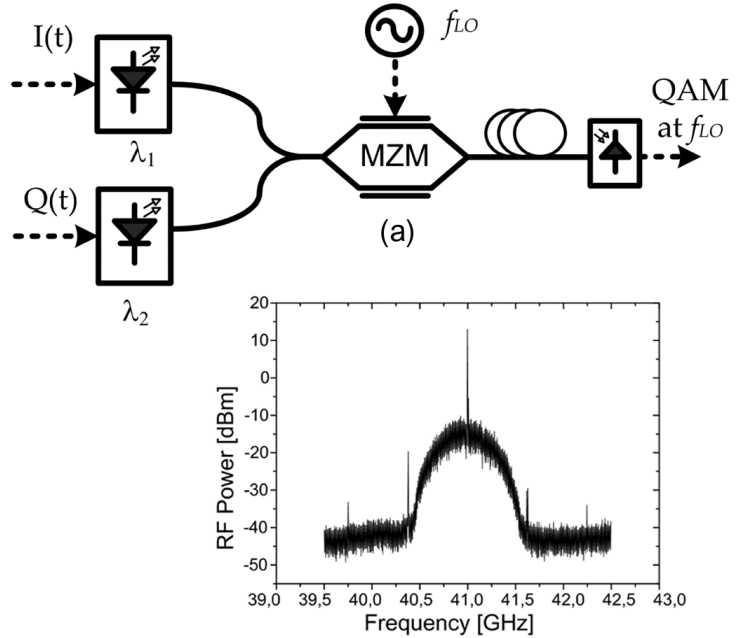

(b)

Fig. 1. Schematic of the proposed (a) PVM architecture [11] and (b) the resulting RF spectrum.

in the generated modulated mm-wave carrier spectrum. Finally, conclusions are provided in Section V.

\section{PVM ARCHITECTURE DESCRIPTION}

\section{A. Architecture Description}

Unlike other proposed PVM [9], [10] architectures involving two or more electrooptical modulators, complicated electronics, and hybrids at the carrier frequency, the PVM architecture proposed utilizes two baseband modulated optical carriers, one single electrooptical modulator, and a dispersive medium like an optical fiber.

In this architecture, shown in Fig. 1, two optical carriers with wavelengths $\lambda_{1}$ and $\lambda_{2}$ are directly modulated using two independent baseband data streams $I(t)$ and $Q(t)$, respectively. The two optical carriers are combined, and an LO carrier at mm-wave frequency modulates the two optical carriers using a single Mach-Zehnder modulator (MZM) biased at its quadrature bias point. Later, the two optical carriers are transmitted through a dispersive element like an optical fiber prior to photodetection. Due to the chromatic dispersion of the fiber, a differential delay $\Delta \tau=D \cdot L \cdot \Delta \lambda$ among the optical carriers is introduced [11], which depends on the wavelength spacing $\Delta \lambda$ (nm), the fiber length $L(\mathrm{~km})$, and the fiber dispersion parameter $(D \mathrm{ps} / \mathrm{km} \cdot \mathrm{nm})$. A $90^{\circ}$ phase shift is induced between the adjacent wavelengths by setting $\Delta \tau=T_{\mathrm{LO}} / 4$, where $T_{\mathrm{LO}}(\mathrm{s})$ is the period of the LO signal. At the PD output, two replicas of the LO with differential phase shift of $90^{\circ}$ are obtained.

One of the limitations of the above mentioned architecture is the high LO component at the electrical spectrum output of the photodiode as shown in Fig. 1(b). This feature limits the system dynamic range thus reducing the wireless system maximum transmission range.

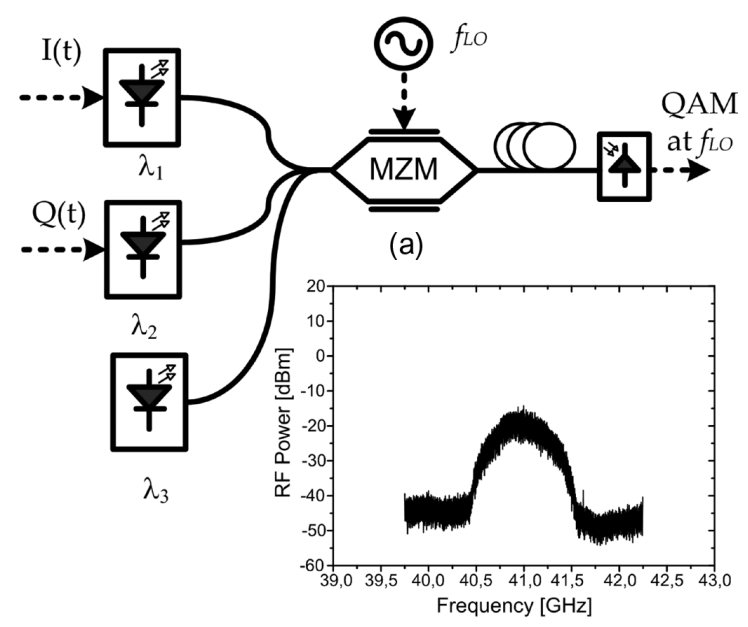

(b)

Fig. 2. Schematic of the PVM architecture with (a) LO suppression and (b) the corresponding electrical spectrum.

\section{B. LO Suppression}

The architecture proposed to suppress the unwanted LO component, shown in Fig. 2, is the same as the original architecture but with an added third continuous wave laser emitting at wavelength $\lambda_{3}$. The three optical carriers are externally modulated by a mm-wave LO carrier and transmitted through an optical fiber of length $L$ before photodetection.

As previously, a $90^{\circ}$ phase shift is induced between the $I$ and $Q$-modulated LO components by setting the relative delay due to the fiber's chromatic dispersion between $\lambda_{1}$ and $\lambda_{2}$ as $\tau_{21}=D \cdot L \cdot \Delta \lambda_{21}=T_{\mathrm{LO}} / 4$, where $T_{\mathrm{LO}}[\mathrm{s}]$ is the time period of the LO carrier. Other than the delay between the $I$ - and $Q$-modulated LO components, there is a delay induced between the $I$-modulated optical carrier and the continuous-wave (CW) optical carrier $\tau_{31}=D \cdot L \cdot \Delta \lambda_{31}$ which results in the suppression of the unwanted LO component at the photodiode output. The following section describes the conditions required by the third laser to satisfy the conditions required for suppressing the LO component.

\section{THEORETICAL MODELING}

In this section, the photo current, noise current, and the SNR of the mm-wave signals generated using the analyzed PVM architecture are derived.

\section{A. Photocurrent}

In the PVM system shown in Fig. 1(a), where two digital signals [non-return-to-zero (NRZ) bipolar signal, $I(t)=B$ or $-B$ amperes with equal probability] are driving two lasers to two different optical power levels above threshold $P_{\mathrm{ON}}$ and $P_{\mathrm{OFF}}$ which satisfy the relations

$$
\begin{aligned}
& P_{\mathrm{ON}}-P_{\mathrm{OFF}}=2 \eta B \\
& P=\frac{P_{\mathrm{ON}}+P_{\mathrm{OFF}}}{2}
\end{aligned}
$$


where $P$ is the mean optical power at the output of any of the two lasers and $\eta$ is the laser internal efficiency.

The dispersion of the fiber is modeled as

$$
\begin{aligned}
& H(\Omega)=\frac{1}{\sqrt{L_{f}}} e^{j \beta(\Omega)} \\
& \beta(\Omega)=\beta_{0}+\beta_{1}\left(\Omega-\Omega_{0}\right)+\frac{\beta_{2}}{2}\left(\Omega-\Omega_{0}\right)^{2}
\end{aligned}
$$

where $\beta_{1}$ is the group delay at the optical frequency $\Omega_{0}$, and $\beta_{2}$ is related to the dispersion parameter of the fiber as

$$
\beta_{2}=-\frac{D \lambda_{0}^{2}}{2 \pi c}
$$

Then, according to [15], the LO component at the photodiode output will be

$$
\begin{aligned}
i_{\mathrm{LO}}(t)= & \Re \frac{1}{L_{\mathrm{tot}}} J_{0}\left(m_{\mathrm{LO}}\right) 2 J_{1}\left(m_{\mathrm{LO}}\right) \cos \left(\frac{\beta_{2} L}{2} \omega_{\mathrm{LO}}^{2}\right) \\
& \times\left[[P+\eta I(t)] \cos \left(\omega_{\mathrm{LO}} t\right)+\left[P+\eta Q\left(t-\tau_{21}\right)\right]\right. \\
& \left.\quad \times \cos \left(\omega_{\mathrm{LO}}\left(t-\tau_{21}\right)\right)\right]
\end{aligned}
$$

where $J_{0}$ is the zeroth-order Bessel functions of the first kind, and $m_{\mathrm{LO}}=\pi V_{\mathrm{LO}} / V_{\pi}$, with $V_{\mathrm{LO}}$ being the amplitude of the LO tone at each MZM electrode and $V_{\pi}$ being the MZM halfwave voltage, $\omega_{\mathrm{LO}}$ is the carrier angular frequency, $\Re$ is the photodiode responsivity, $L_{\text {tot }}$ are the optical losses from laser output to photodiode input when the MZM is biased as its maximum transmission point (the 3-dB extra losses due to the MZM quadrature bias are not considered in this parameter but in the rest of the equation) and where the MZM model considers only the sidebands at $f_{\mathrm{LO}}$ around each optical carrier neglecting the dc terms. $\tau_{21}=-\beta_{2}\left(\Omega_{2}-\Omega_{1}\right)$ is the differential delay induced in the optical carriers due to the fiber's chromatic dispersion. When the length of the fiber and the wavelength spacing are chosen to fulfill the quadrature condition defined as

$$
\tau_{21}=-\beta_{2} L\left(\Omega_{2}-\Omega_{1}\right)=\frac{\tau_{\mathrm{LO}}}{4}=\frac{1}{4 f_{\mathrm{LO}}} .
$$

Then the photocurrent is given as

$$
\begin{aligned}
i_{\mathrm{LO}}(t)=\Re & \frac{1}{L_{\mathrm{tot}}} J_{0}\left(m_{\mathrm{LO}}\right) 2 J_{1}\left(m_{\mathrm{LO}}\right) \cos \left(\frac{\beta_{2} L}{2} \omega_{\mathrm{LO}}^{2}\right) \\
\times & {[P+\eta I(t)] \cos \left(\omega_{\mathrm{LO}} t\right) } \\
& \left.+\left[P+\eta Q\left(t-\frac{1}{4 f_{\mathrm{LO}}}\right)\right] \sin \left(\omega_{\mathrm{LO}} t\right)\right] .
\end{aligned}
$$

According to (8), there is a small delay in $Q(t)$ with respect to $I(t)$ which can be neglected as long as $f_{\mathrm{LO}}$ is much greater than the symbol rate. When the $I$-branch optical carrier is delayed by $\left(1 / 4 f_{\mathrm{LO}}\right)$ with respect to the $Q$-branch optical carrier, the corresponding baseband signals are also delayed. For instance, if $f_{\mathrm{LO}}=20 \mathrm{GHz}$ and baseband data rate is $1 \mathrm{~Gb} / \mathrm{s}$ per branch $(2$ $\mathrm{Gb} / \mathrm{s}$ totally), the delay is $12.5 \mathrm{ps}$ while the symbol rate is 1000 ps. So a $1.25 \%$ differential time shifting is induced between the $I$ and $Q$ demodulated branch. This small relative delay between digital $I$ and $Q$ branches could be easily corrected, if needed, by inserting a differential delay in the optical path between both lasers and the combiner or by inserting a differential electrical delay between the digital streams before driving the lasers.

As it happens in any analog optical link, the dispersion of the optical link would induce the total suppression of the LO carrier after photo detection if double-sideband optical modulation is used and the dispersion-induced phase shift between the optical carrier and the modulated sideband is $180^{\circ}$. In our case, when the quadrature condition from (7) is fulfilled the dispersive attenuation term is reduced to the expression

$$
\cos \left(\frac{\beta_{2} L}{2} \omega_{\mathrm{LO}}^{2}\right)=\cos \left(\frac{\pi}{4} \frac{\omega_{\mathrm{LO}}}{\Omega_{2}-\Omega_{1}}\right) .
$$

Thus, for the dispersive attenuation term to be significant, the optical carriers spacing $\left(\Omega_{2}-\Omega_{1}\right)$ should be lower than the LO angular frequency which is not a possible situation as it would imply that the modulation sidebands of both optical carriers would be interleaved in the frequency domain generating undesired beat components at the photodiode output.

Taking into account both considerations, the final expression for the LO component at the photodiode output is

$$
\begin{aligned}
& i_{\mathrm{LO}}(t) \approx \Re \frac{1}{L_{\mathrm{tot}}} J_{0}\left(m_{\mathrm{LO}}\right) 2 J_{1}\left(m_{\mathrm{LO}}\right) \\
& \quad \times\left[[P+\eta I(t)] \cos \left(\omega_{\mathrm{LO}} t\right)+[P+\eta Q(t)] \sin \left(\omega_{\mathrm{LO}} t\right)\right] .
\end{aligned}
$$

\section{B. Noise and Signal-to-Noise Ratio}

The dc component of the photodetected current can be expressed as

$$
i_{\mathrm{DC}}=\frac{\Re P}{L_{\mathrm{tot}}}\left[J_{0}^{2}\left(m_{\mathrm{LO}}\right)+2 J_{1}^{2}\left(m_{\mathrm{LO}}\right)\right]
$$

where $J_{1}$ is the first-order Bessel functions of the first kind.

The three most important noise sources at the photoreceiver output will be the laser relative intensity noise (RIN), the photodiode shot noise, and the thermal noise at the photodetector output including the preamplifier contribution. The variance of these three noise contributions in terms of detected current are

$$
\begin{aligned}
\sigma_{\mathrm{RIN}}^{2} & =\operatorname{RIN} i_{\mathrm{DC}}^{2} \Delta f \\
& =\operatorname{RIN}\left(\frac{\Re P}{L_{\text {tot }}}\left[J_{0}^{2}\left(m_{\mathrm{LO}}\right)+2 J_{1}^{2}\left(m_{\mathrm{LO}}\right)\right]\right)^{2} \Delta f \quad(12) \\
\sigma_{\text {shot }}^{2} & =2 q\left(i_{\mathrm{DC}}+i_{\mathrm{dark}}\right) \Delta f \\
& =2 q\left(\frac{\Re P}{L_{\text {tot }}}\left[J_{0}^{2}\left(m_{\mathrm{LO}}\right)+2 J_{1}^{2}\left(m_{\mathrm{LO}}\right)\right]+i_{\text {dark }}\right) \Delta f
\end{aligned}
$$

$\sigma_{\text {thermal }}^{2}=\frac{4 k T \Delta f}{R_{L}} F$

where RIN is the RIN parameter of both lasers, which is considered identical, $\Delta f$ is the electrical bandwidth of the data signal around LO carrier, $q=1.610^{-19} \mathrm{C}$ is the electron charge, $i_{\text {dark }}$ is the dark current of the photodiode, $\mathrm{k}=1.310^{-23} \mathrm{~J} / \mathrm{K}$ is the 
Boltzmann constant, $T$ is the absolute temperature of the photodetector in Kelvin, $R_{L}$ is the load resistance at the photodetector output, and $F$ is the noise figure of the preamplifier. From the above noise components, the SNR of the PVM is expressed as (15), shown at the bottom of the page, where $P_{\text {data }}$ is the power of the signal generated around LO carrier at the photodetector output once the power corresponding to the LO tone has been subtracted.

\section{SNR of LO Suppressed PVM}

The expression for the photocurrent corresponding to the modified PVM architecture shown in Fig. 2(c) is as follows:

$$
\begin{gathered}
i_{\mathrm{LO}}(t) \approx \Re \frac{1}{L_{\mathrm{tot}}} J_{0}\left(m_{\mathrm{LO}}\right) 2 J_{1}\left(m_{\mathrm{LO}}\right)\left[[P+\eta I(t)] \cos \left(\omega_{\mathrm{LO}} t\right)\right. \\
\left.+[P+\eta Q(t)] \sin \left(\omega_{\mathrm{LO}} t\right)+P_{3} \cos \left(\omega_{\mathrm{LO}}\left(t-\tau_{31}\right)\right)\right]
\end{gathered}
$$

where $P_{3}$ is the optical power at the third laser output and $\tau_{31}=$ $-\beta_{2}\left(\Omega_{3}-\Omega_{1}\right)$ is the differential delay induced by the fiber's chromatic dispersion between the optical carriers $\lambda_{3}$ and $\lambda_{1}$.

To suppress the undesired high LO component at the photodetector output, the following conditions need to be satisfied:

$$
\begin{aligned}
\tau_{31} & =2.5 \tau_{21}=5 \frac{\tau_{\mathrm{LO}}}{8}=\frac{5}{8 f_{\mathrm{LO}}} \\
P_{3} & =P \sqrt{2}
\end{aligned}
$$

Substituting these results in (16), the resulting photocurrent is

$$
\begin{aligned}
i_{\mathrm{LO}}(t) \approx & \Re \frac{1}{L_{\mathrm{tot}}} J_{0}\left(m_{\mathrm{LO}}\right) 2 J_{1}\left(m_{\mathrm{LO}}\right) \\
\times & {\left[P \sqrt{2} \cos \left(\omega_{\mathrm{LO}} t-\frac{\pi}{4}\right)+\eta I(t) \cos \left(\omega_{\mathrm{LO}} t\right)\right.} \\
& \left.\quad+\eta Q(t) \sin \left(\omega_{\mathrm{LO}} t\right)+P \sqrt{2} \cos \left(\omega_{\mathrm{LO}} t-\frac{5 \pi}{4}\right)\right] \\
= & \Re \frac{1}{L_{\mathrm{tot}}} J_{0}\left(m_{\mathrm{LO}}\right) 2 J_{1}\left(m_{\mathrm{LO}}\right) \\
& \times\left[\eta I(t) \cos \left(\omega_{\mathrm{LO}} t\right)+\eta Q(t) \sin \left(\omega_{\mathrm{LO}} t\right)\right]
\end{aligned}
$$

where a pure QAM signal is obtained.

The noise contributions to the signal are the same as in the previous case except the addition of another laser RIN source. The dc current at the photodetector output is given as

$$
\begin{aligned}
i_{\mathrm{DC}} & =\frac{\Re P^{\prime}}{L_{\mathrm{tot}}}\left[J_{0}^{2}\left(m_{\mathrm{LO}}\right)+2 J_{1}^{2}\left(m_{\mathrm{LO}}\right)\right] \\
P^{\prime} & =P\left(1+\frac{1}{\sqrt{2}}\right) .
\end{aligned}
$$

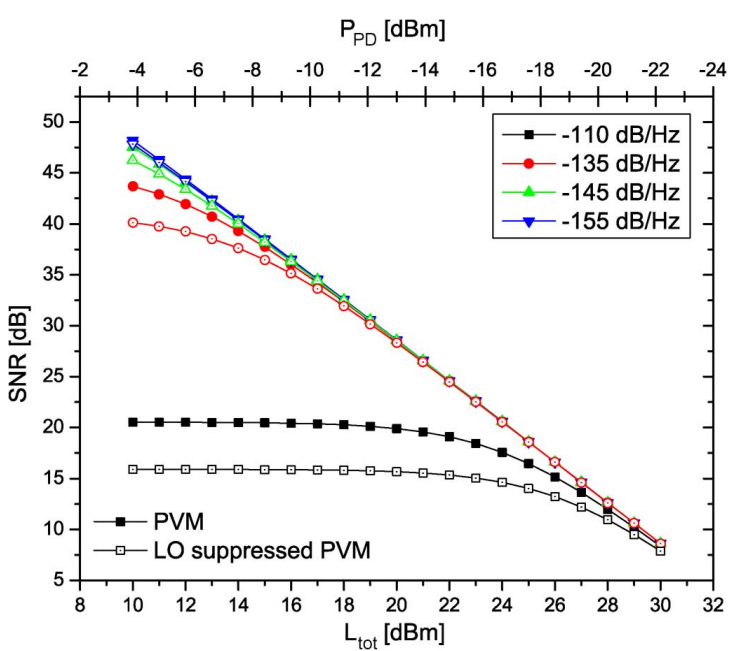

Fig. 3. SNR versus photodiode input power plotted against various laser RIN values. The filled blocks correspond to the QAM signal containing the LO peak and the empty blocks correspond to the LO suppressed QAM.

The increment of dc current by a factor of 1.7 increases the RIN noise by $4.6 \mathrm{~dB}$, and the shot noise by $2.3 \mathrm{~dB}$ with no effect on the thermal noise contribution.

The effect of noise on the system performance can be analysed by calculating the photodiode input power tolerance. The photodiode input power was varied by changing the $L_{\text {tot }}$, PVM parameter and the SNR plotted for various laser RIN values as shown in Fig. 3.

From Fig. 3, it can be observed that in a system with low RIN noise, the SNR is higher for high photodiode input power, but as the power impinging the photodiode reduces, the thermal noise dominates and the SNR is reduced. However, in a RIN dominant scenario the SNR remains constant for certain values of input power, i.e., the SNR cannot be increased by increasing the input power to the photodiode. It should be noted that the input power to the photodiode $P_{\mathrm{PD}}$ in the LO suppressed PVM is $3 \mathrm{~dB}$ lower because of an additional $3 \mathrm{~dB}$ coupler used to add the third laser. This can be avoided by using a WDM coupler instead of cascaded 3-dB couplers. In the expressions of SNR, the $3-\mathrm{dB}$ loss due to the extra coupler is neglected.

Also the performance of the system on the LO tone power is evaluated by varying the parameter $m_{\mathrm{LO}}$. Table I describes the values of the various device parameters considered it calculating the SNR.

$$
\begin{aligned}
\frac{S}{N} & =\frac{P_{\text {data }}}{\sigma_{\mathrm{RIN}}^{2} R_{L}+\sigma_{\text {shot }}^{2} R_{L}+\sigma_{\text {thermal }}^{2} R_{L}} \\
& =\frac{\left(2 \Re J_{0}^{2}\left(m_{\mathrm{LO}}\right) J_{1}^{2}\left(m_{\mathrm{LO}}\right)\right)^{2} \eta^{2} B^{2}}{R I N\left(\Re P\left[J_{0}^{2}\left(m_{\mathrm{LO}}\right)+2 J_{1}^{2}\left(m_{\mathrm{LO}}\right)\right]\right)^{2} \Delta f+2 q\left(\Re P\left[J_{0}^{2}\left(m_{\mathrm{LO}}\right)+2 J_{1}^{2}\left(m_{\mathrm{LO}}\right)\right]+i_{\text {dark }}\right) \Delta f+\frac{L_{\text {tot }} 4 k T \Delta f F}{R_{L}}}
\end{aligned}
$$


TABLE I

DeVice Parameters of the PHotonic Vector Modulator

\begin{tabular}{|l|l|}
\hline Device parameter & Value \\
\hline$P$ (Laser) & $+6 \mathrm{dBm}$ \\
\hline$R I N$ & $-135 \mathrm{~dB} / \mathrm{Hz}$ \\
\hline$\eta$ & $0.24 \mathrm{~W} / \mathrm{A}$ \\
\hline Apeak & $25 \mathrm{~mA}(\mathrm{Vp}-\mathrm{p}=2.5 \mathrm{~V})$ \\
\hline$\Re$ & $0.65 \mathrm{~A} / \mathrm{W}$ \\
\hline$i_{\text {dark }}$ & $40 \mathrm{pA}$ \\
\hline $\mathrm{R}_{\mathrm{L}}$ & $50 \mathrm{Ohms}$ \\
\hline$\Delta \mathrm{f}$ & $1 \mathrm{GHz}$ \\
\hline $\mathrm{L}_{\mathrm{tot}}$ & $10 \mathrm{~dB}$ \\
\hline $\mathrm{F}$ & $4.5 \mathrm{~dB}$ \\
\hline
\end{tabular}

$$
\mathrm{V}_{\mathrm{LO}} \mathrm{N} \pi
$$

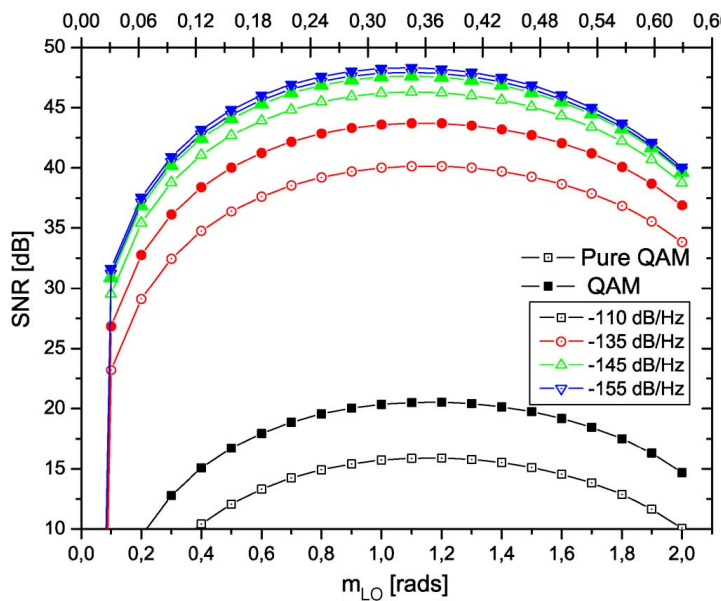

Fig. 4. SNR versus $m_{\mathrm{LO}}$ plotted against various laser RIN values. The filled blocks correspond to the QAM signal containing the LO peak and the empty blocks correspond to the LO suppressed QAM.

Similarly, the SNR of the signal generated with a $m_{\mathrm{LO}}$ of 1.2 is calculated by varying the laser power $P$ and the data current $A_{\text {peak }}$ simultaneously. Both the parameters were varied simultaneously to simulate the laser available in the laboratory and also to maintain near $100 \%$ optical modulation index.

From Fig. 4, it can be seen that the optimum SNR is obtained by modulation with an $\mathrm{LO}$ tone resulting in a $m_{\mathrm{LO}}$ of 1.2 . The dependence of SNR on the laser power with near $100 \%$ modulation index can be seen in Fig. 5. In a lower laser RIN noise scenario, the increase of the laser power increases the SNR considerably, whereas in a RIN dominant case, the SNR of both the QAM signals (with and without the LO peak) have the SNR constant. In an RIN dominant case, the LO suppressed QAM signal is expected to have $4.6 \mathrm{~dB}$ more noise power. This result can be checked in Fig. 5 for a laser RIN noise of $-110 \mathrm{~dB} / \mathrm{Hz}$, the SNR between the two PVM options is around $4.6 \mathrm{~dB}$.

The effect of the baseband data bandwidth on the SNR of the QAM signal is plotted in Fig. 6. With increasing the bandwidth, the RIN noise, the shot noise, and the thermal noise increase according to (12) to (14). From the SNR results depicted in Figs. 3-6, it could be stated that the proposed PVM architecture in its two options is able to generate QPSK signals with data rates up to $10 \mathrm{~Gb} / \mathrm{s}$ (corresponding to baseband data bandwidth around $5 \mathrm{GHz}$ ) with SNR from 35 to $50 \mathrm{~dB}$ if the proper

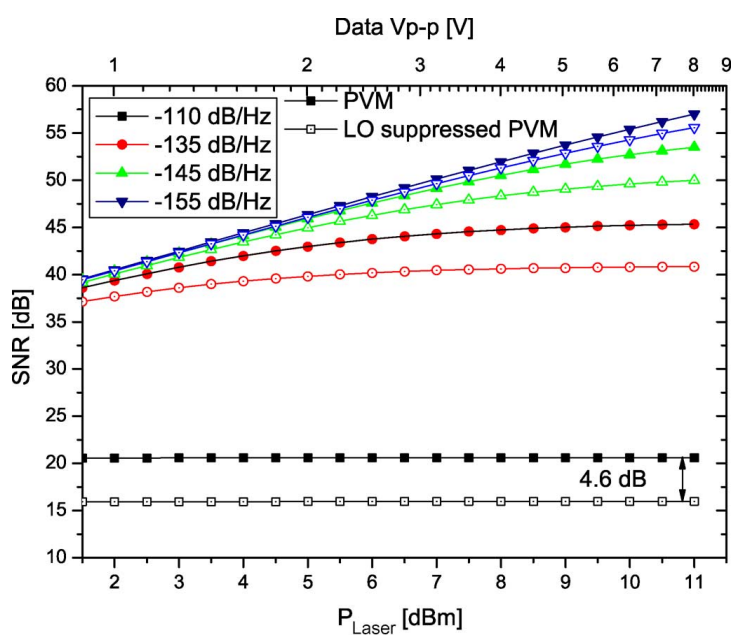

Fig. 5. SNR versus laser output power with near $100 \%$ modulation index. The filled blocks correspond to the QAM signal containing the LO peak and the empty blocks correspond to the LO suppressed QAM.

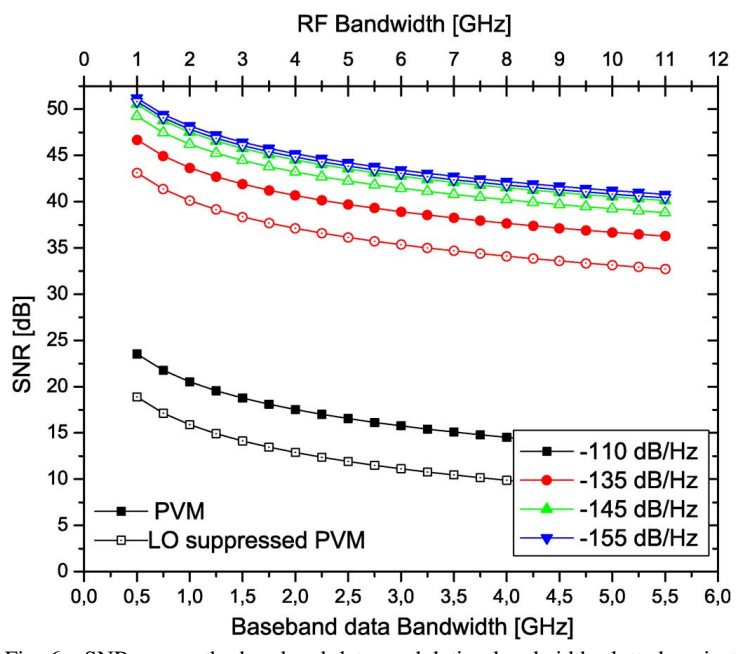

Fig. 6. SNR versus the baseband data modulation bandwidth plotted against different laser RIN levels. The filled blocks correspond to the QAM signal containing the LO peak and the empty blocks correspond to the LO suppressed QAM.

components and parameters are selected. These SNR values correspond to practically error-free QPSK signals.

\section{EXPERIMENTAL RESULTS}

\section{A. Experimental Setup}

Two DFB semiconductor lasers (wavelength, $\lambda_{1}=$ $1550.12 \mathrm{~nm}, \lambda_{2}=1549.02 \mathrm{~nm}$ ) of $1-\mathrm{GHz}$ modulation bandwidth were directly current modulated with $625-\mathrm{Mb} / \mathrm{s}$ data for generating a 1.25-Gb/s QPSK signal at a LO frequency of $41 \mathrm{GHz}$. Two independent data streams from a PRBS were amplified to $2.5 \mathrm{~V}_{\mathrm{p}-\mathrm{p}}$ and directly modulated on the two DFB lasers. Due to the circuitry of the DFB lasers, the bias current of the lasers was dependent on the modulating current, and 

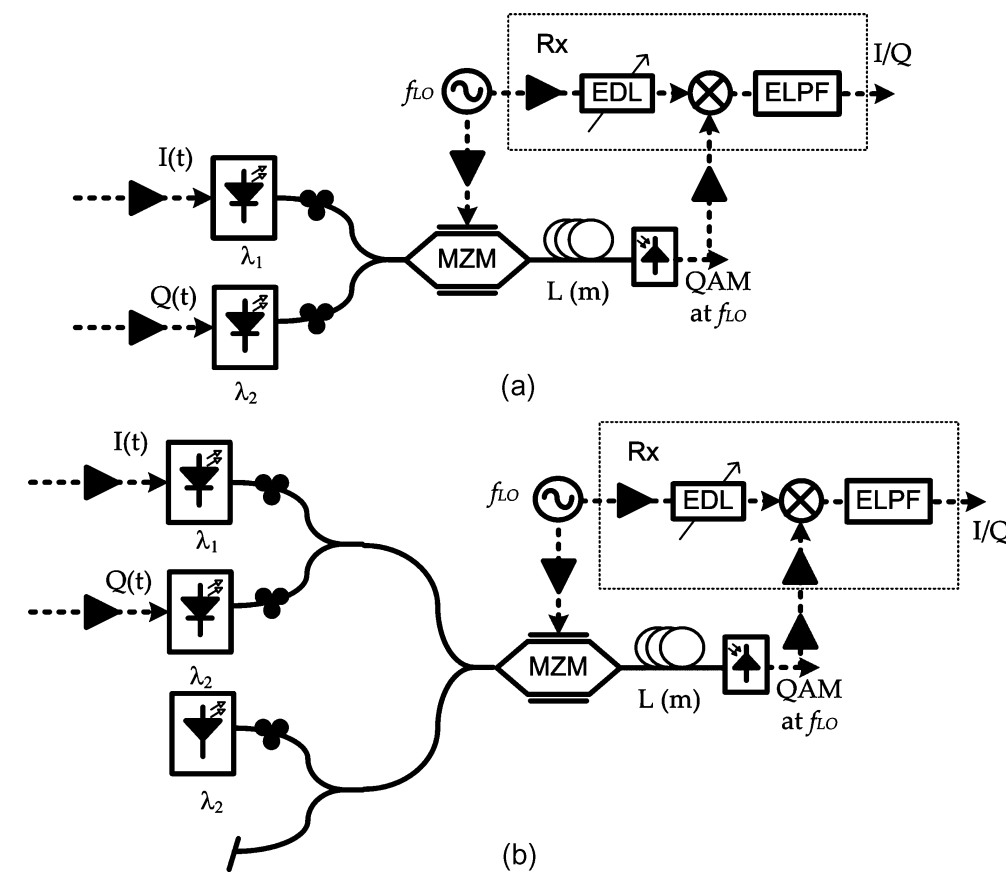

Fig. 7. (a) Experimental setup of the PVM architecture, and (b) the PVM experimental setup for generating LO suppressed RF signals.

at $2.5 \mathrm{~V}_{\mathrm{p}-\mathrm{p}}$, the two DFB lasers were emitting $+6 \mathrm{dBm}$ of power. The two optical signals were polarization controlled and combined using an optical $3 \mathrm{~dB}$ coupler. The corresponding optical signal was externally modulated with a $41-\mathrm{GHz} \mathrm{LO}$ tone amplified to $+13 \mathrm{dBm}$ using a $50-\mathrm{GHz}$ MZM biased at its quadrature bias point.

For generating the $90^{\circ}$ phase shift between the LO components on the two optical carriers, the combined optical signals were transmitted through a single mode fiber $(D=16.5 \mathrm{ps}-\mathrm{nm} / \mathrm{km})$ of length $L=340 \mathrm{~m}$ and photodetected using a $45 \mathrm{GHz}$ photodiode with a responsivity of $0.65 \mathrm{~W} / \mathrm{A}$. The PVM generatedQPSK signals were demodulated using a simple electrical receiver. The generated RF signal was amplified to $+10 \mathrm{dBm}$ and mixed (mixer with conversion loss of $6 \mathrm{~dB}$ ) with a $+13 \mathrm{dBm}$ phase corrected $\mathrm{LO}$ component. By changing the phase of the $\mathrm{LO}$ using an electrical delay line, the $I$ and $Q$ components were demodulated. An electrical low pass filter with a cutoff frequency of $1.87 \mathrm{GHz}$ (due to the lack of low-pass filters at baseband frequency) was used to downconvert the demodulated $I$ and $Q$ components. The baseband demodulated $I$ and $Q$ were analysed using a DCA and the $Q$ factors were measured.

Similarly for generating the LO suppressed QPSK, a third continuous-wave DFB laser at wavelength $1547.37 \mathrm{~nm}$ was added to the original setup using a cluster of optical 3-dB couplers as shown in Fig. 7(b). At the receiver, a second electrical amplifier is used to amplify the signal sufficiently prior to mixing for demodulation.

\section{B. Results Analysis: Performance Comparison}

To validate the theory mentioned in the previous section, the experiments were performed to analyze the system performance limitations. Two main parameters were considered in analyzing the limitations of the system performance. One the baseband data current (varied by simultaneously changing the laser output power), and the LO power to the MZM. From (11), it can be observed that the data current $(B)$ and the $\mathrm{LO}$ (through $m_{\mathrm{LO}}$ ) power to the MZM determine the data power of the QAM signal. Thus, it is important to know to what limits these powers can reach without inducing any degradations to the system performance.

By varying the $I$ and $Q$ data power, the optical power was also varying because of the lasers internal bias circuitry. By doing so at different data power levels the optical power was changed corresponding to a near $100 \%$ optical modulation index. While analyzing the effect of the data power on the system performance, the $\mathrm{LO}$ power was maintained at $+13 \mathrm{dBm}$. The laser power was varied from +1 to $+11 \mathrm{dBm}$. Fig. 8 contains the results obtained. The $Q$ factor of the demodulated signals was measured using a digital communication analyzer.

As shown in Fig. 8, the $Q$ factor of the demodulated $I$ and $Q$ data streams at low laser output power is very poor because of the poor SNR of the generated RF signal. But at higher modulating data current and laser output power, the $Q$ factor is seen degraded even though the transmitted signal shows to have a higher SNR according to Fig. 5. This degradation in the $Q$ factor is due to the distortion induced in the laser due to high data current, which can be seen in the eye diagram in Fig. 8 inset. The best available $Q$ factors for both the PVM architectures was obtained at a laser output power of $+6 \mathrm{dBm}$ when driven with data of $2.5 \mathrm{~V}_{\mathrm{p}-\mathrm{p}}$. Fig. 9 shows the eye diagrams of the demodulated $I$ and $Q$ signals of the RF signal generated with the LO suppressed PVM. The $Q$ factors were measured as 7.8 


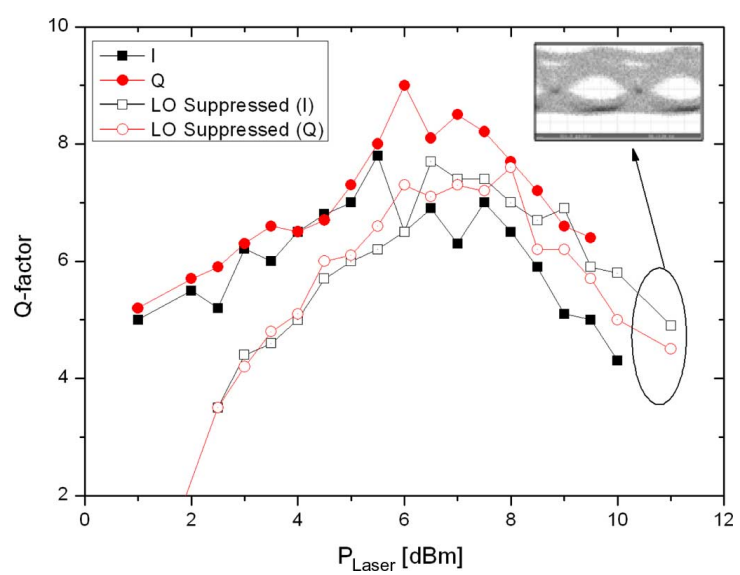

Fig. 8. Measured $Q$ factor plotted against the laser output power with near $100 \%$ modulation index.

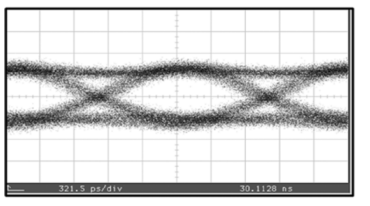

(a)

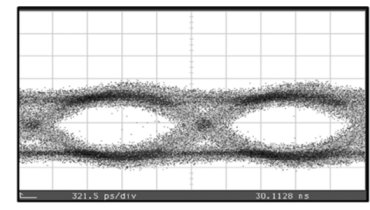

(b)
Fig. 9. I and $Q$ eye diagrams for the LO-suppressed PVM architecture.

$(I), 8(Q)$ for the PVM architecture, and $7.7(I), 7.3(Q)$ for LO-suppressed PVM architecture corresponding to estimated bit-error-rate (BER) values of $3 \cdot 10^{-15}(I) 6 \cdot 10^{-16}(Q)$ for the PVM architecture and $7 \cdot 10^{-15}(I), 1 \cdot 10^{-13}(Q)$ for LO-suppressed PVM architecture.

The effect of LO power to the MZM on the demodulated $I$ and $Q$ data streams is also experimentally verified by varying the $\mathrm{LO}$ power and measuring the $Q$ factors of the demodulated signals. For doing so the directly modulated lasers were driven for optimum performance using the operating points derived from the calculations plotted in Fig. 4.

From Fig. 10, it can be noted that at lower LO power the generated signal has a poor SNR, but the optimum $Q$ factor was obtained at an LO power of $10 \mathrm{dBm}$ which corresponds to $m_{\mathrm{LO}}$ of 1.05 with an MZM $V_{\pi}$ of $6 \mathrm{~V}$. Due to the lack of availability of a power amplifier able to generate high power, the LO could be tuned only up to $+14 \mathrm{dBm}$.

\section{CONCLUSION}

In this paper, the performance analysis of a photonic vector modulator architecture for generating high capacity multilevel mm-wave wireless carriers is presented. The PVM technique originally reported in [11] was modified by introducing a third $\mathrm{CW}$ laser in order to suppress the LO peak completely. LO component suppression is achieved by adjusting the third laser driving conditions.

The two configurations' theoretical model has been obtained and the influence of the PVM system parameters on the quality of the QAM generated signal has been analyzed in terms of the

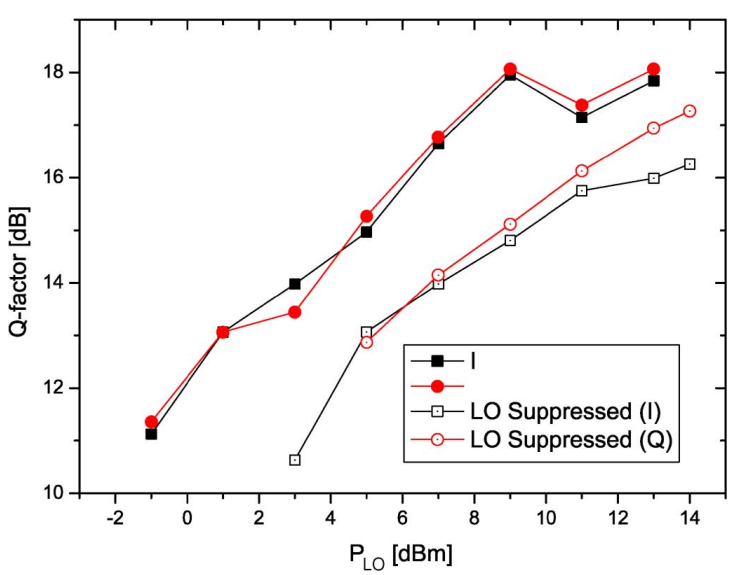

Fig. 10. Measured $Q$ factor plotted against the LO power.

signal-to-noise ratio of the generated signal. The simulation results show that very good quality QAM signals with SNR better than $35 \mathrm{~dB}$ can be generated with a proper selection of system parameters.

The maximum theoretical difference in SNR between the two techniques is $4.6 \mathrm{~dB}$ in an RIN dominant scenario which is the typical case. The experimental results show that very good quality QAM signal are obtained in both cases with BER values estimated to be below $10^{-13}$ when electrical demodulation is used to receive the QAM signals generated with the proposed PVM architecture. Even though the modified PVM option is more noisy that the original PVM architecture, it is important to state that it generates a QAM signal more adequate to current digital wireless systems increasing at the same time the dynamic range of all the electrical components between the photodetector output and the transmitter antenna.

The feasibility of pure QAM/QPSK $1.25-\mathrm{Gb} / \mathrm{s} \mathrm{mm-wave}$ mm-wave carriers has been demonstrated. Photonic generation of multilevel modulated mm-wave carriers can overcome several limitations of purely electrical systems in terms of bandwidth and flexibility, thanks to the inherent advantages of photonic components and optical fibers, being a candidate to implement infrastructures for the wireless extension of broadband wireline access networks (e.g., FTTH) with 10-Gb/s capacity.

\section{REFERENCES}

[1] C. P. Liu et al., "Full-duplex DOCSIS/wirelessDOCSIS fiber-radio network employing packaged AFPMs as optical/electrical transducers," $J$. Lightw. Technol., vol. 25, no. 3, pp. 673-684, 2007.

[2] M. A. Piqueras, V. Polo, and J. Marti, "Simultaneous base-band and mm-wave delivery of Gbps data employing photonic vector modulators," presented at the Optical Fiber Communication Conference (OFC), Anaheim, CA, 2007, Paper JThA60.

[3] C. Lim et al., "Millimeter-wave broadband fiber-wireless system incorporating baseband data transmission over fiber and remote $\mathrm{LO}$ delivery," J. Lightw. Technol., vol. 18, no. 10, pp. 1355-1363, Oct. 2000.

[4] K. Ikeda, T. Kuri, and K. Kitayama, "Simultaneous three-band modulation and fiber-optic transmission of $2.5-\mathrm{Gb} / \mathrm{s}$ baseband, microwave-, and 60-GHz-band signals on a single wavelength," J. Lightw. Technol., vol. 21, no. 12, pp. 3194-3202, Dec. 2003.

[5] A. J. Seeds and J. K. Williams, "Microwave photonics," J. Lightw. Technol., vol. 24, no. 12, pp. 4628-4641, 2006. 
[6] M. Hirata et al., "120-GHz wireless link using photonic techniques for generation, modulation, and emission of millimeter-wave signals," $J$. Lightw. Technol., vol. 21, no. 10, pp. 2145-2153, Oct. 2003.

[7] J. Yu et al., "Optical millimeter-wave generation or up-conversion using external modulators," IEEE Photon. Technol. Lett., vol. 18, no. 1, pp. 265-267, 2006.

[8] A. Wiberg et al., "Fiber-optic 40-GHz mm-wave link with $2.5-\mathrm{Gb} / \mathrm{s}$ data transmission," IEEE Photon. Technol. Lett., vol. 17, no. 9, pp. 1938-1940, 2005.

[9] W. D. Jemison, A. J. Kreuzberger, and E. Funk, "Microwave photonic vector modulator for high-speed wireless digital communications," IEEE Microw. Compon. Lett., vol. 12, no. 4, pp. 125-127, 2002.

[10] P. Candelas et al., "Optically generated electrical-modulation formats in digital-microwave link applications," J. Lightw. Technol., vol. 21, no. 2, pp. 496-499, 2003.

[11] M. A. Piqueras et al., "Direct photonic generation of electrical vector modulations at microwave/millimeter-wave frequencies," IEEE Photon. Technol. Lett., vol. 17, no. 9, pp. 1947-1949, 2005.

[12] R. Sambaraju et al., "Generation of multi-Gbps MQAM/MPSK modulated mm-wave carriers employing photonic vector modulator techniques," J. Lightwave Tecnol., vol. 25, no. 11, pp. 3350-3357, 2007.

[13] R. Sambaraju et al. "Photonic vector modulation of $3.6 \mathrm{~Gb} / \mathrm{s} 16 \mathrm{QAM}$ at $39 \mathrm{GHz}$ for radio-on-fibre systems," presented at the Eur. Conf. Optical Communications (ECOC), Berlin, Germany, Sep. 2007, Tu5.4.7.

[14] J. L. Corral et al., "Pure QAM signal generation with photonic vector modulator," presented at the Optical Fiber Communication Conf. (OFC), San Diego, CA, 2008, Paper OThP1.

[15] J. L. Corral, J. Marti, and J. M. Fuster, "General expressions for IM/DD dispersive analog optical links with external modulation or optical up-conversion in a Mach-Zehnder electrooptical modulator,' IEEE Trans. Microw. Theory Tech., vol. 49, no. 10, pp. 1968-1976, 2001 .

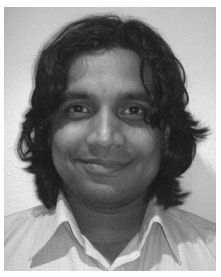

Rakesh Sambaraju (S'07) was born in Shadnagar, India, in 1980. He received the Bachelor's of Technology degree in electronics and communications engineering (with distinction) from the Jawaharlal Nehru Technical University, Hyderabad, India, in 2002 and the Master's of Science (M.Sc) degree in telecommunications specialized in optical communication systems from the Technical University of Denmark, Denmark, in 2006. He is currently working towards the Ph.D. degree at the Universidad Politécnica de Valencia (UPV), Spain.

$\mathrm{He}$ is a member of the Valencia Nanophotonics Technology Centre (NTC) of UPV, where he is currently participating in the FP6 project IPHOBAC, FP7 project HELIOS, and the national project GODIR. His research interests include optical modulation formats, optical modulators, radio-over-fiber systems, and microwave photonics

Mr. Sambaraju is a student member of the Optical Society of America (OSA).

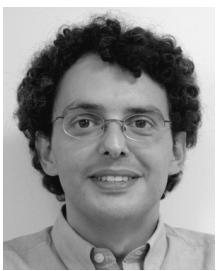

Juan Luis Corral (S'91-A'99-M'01) was born in Zaragoza, Spain, on April 20, 1969. He received the Ingeniero de Telecomunicación degree (with first-class hons.) and the Doctor Ingeniero de Telecomunicación degree from the Universidad Politécnica de Valencia, Spain, in 1993 and 1998, respectively.

During 1993, he was an Assistant Lecturer at the Departamento de Comunicaciones at the Universidad Politécnica de Valencia. From 1993 to 1995, he was at the Microwave Technology and Equipment Section (XRM) at the European Space Research and Technology Centre, ESTEC, The Netherlands, from the European Space Agency (ESA), where he was engaged in research on MMIC-based technologies and photonics technologies for beamforming networks for on-board phased-array antennas. In 1995, he joined the Communications Department at the Universidad Politécnica de Valencia, where he became an Associate Professor in 2000. His research interests include phased-array antennas, optical beamforming networks, and microwave and millimeter-wave optical fiber systems. He has au- thored or coauthored over 70 papers in international journal and conference proceedings in his areas of research interests.

Dr. Corral is a member of several societies of IEEE. He was recipient of the 1998 Doctorate Prize of the Telecommunications Engineer Association in Spain for his $\mathrm{Ph} . \mathrm{D}$. dissertation on applications of MMIC and photonic technologies to phased-array antennas.

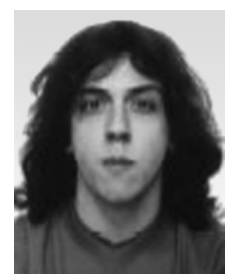

Jesús Palací (S'07) was born in Valencia, Spain, on November 29, 1985. He is with the Nanophotonics Technology Center, Universidad Politécnica de Valencia (UPV), Spain, finishing his final project in order to obtain the Ingeniero de Telecomunicación degree from the Universidad Politécnica de Valencia, Valencia, Spain

His research interests include radio-over-fiber systems, optical modulation formats, and photonic vector modulation/demodulation.

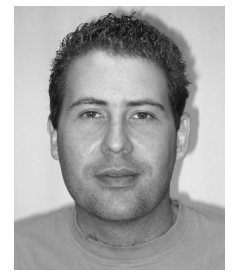

Valentín Polo (M'04) received the Ingeniero de Telecomunicación and Dr. Ingeniero de Telecomunicación (Ph.D.) degree from the Universidad Politécnica de Valencia (UPV), Spain, in 1997 and 2003, respectively.

$\mathrm{He}$ is a member of the Valencia Nanophotonics Technology Centre (NTC), UPV, were he is involved at research and technical management levels in national and European R\&D projects dealing with microwave-photonics and broadband access networks. The main projects he has participated in are FP5 IST-2000 25390 "OBANET" and FP6 IST-1-507781 GANDALF (technical manager). Currently, he is participating in PF6 projects IPHOBAC and ISIS. His research interests in this field include external modulators, photonic-microwave filters, dispersion compensation, and broadband wireline and wireless access technologies and systems, in which he has coauthored over 90 papers in international journals and conferences.

Dr. Polo received the Cátedra Telefónica-UPV 2005 award for his Ph.D. dissertation "Applications of Electro-Absorption Modulators in Radio-over-Fibre systems." He acts as reviewer for several IEE, IEEE, Optical Society of America (OSA), and EU-IST journals and conferences.

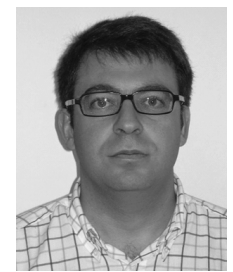

Javier Martí (S'89-M'92) received the Ingeniero de Telecomunicación degree from the Universidad Politécnica de Catalunya, Catalunya, Spain, in 1991 and the Doctor Ingeniero de Telecomunicación degree (Ph.D.) from the Universidad Politécnica de Valencia, Valencia, Spain, in 1994.

During 1989 and 1990, he was an Assistant Lecturer at the Escuela Universitaria de Vilanova, Barcelona, Spain. From 1991 to 2000, he was a Lecturer and Associate Professor at the Telecommunication Engineering Faculty, where he is currently Full Professor. In 2003, he was appointed Director of the Valencia Nanophotonics Technology Centre. He has authored or coauthored over 200 papers in referred international technical journals and hold 12 patents in the fields of broadband hybrid fiber-radio systems and microwave/millimeter-wave photonics, fiber-based access networks, terabit/s OTDM/WDM optical networks, advanced optical processing techniques for microwave signals and ultrahigh-speed data transmission, and (silicon) integrated photonics, including planar photonic crystals and metamaterials. He has participated very actively in the European Research Framework (FP5 and FP6), leading several projects in the areas of broadband radio-over-fiber access (OBANET, GANDALF), all-optical processing in photonic networks (LASAGNE), and nanophotonic logic gates (PHOLOGIC).

Prof. Martí has served as member of the Technical Program Committee of ECOC, LEOS, Microwave Photonics, and other international workshops and conferences. 



\section{Paper D}

Generation of Pure Electrical Quadrature Amplitude Modulation With Photonic Vector Modulator

J. L. Corral, R. Sambaraju, M. A. Piqueras, and V. Polo

Optics Letters, Vol. 33, no. 12, pages 1294-1296, 2008. 


\title{
Generation of pure electrical quadrature amplitude modulation with photonic vector modulator
}

\author{
Juan L. Corral, ${ }^{1, *}$ Rakesh Sambaraju, ${ }^{1}$ Miguel A. Piqueras, ${ }^{2}$ and Valentín Polo ${ }^{1}$ \\ ${ }^{1}$ Valencia Nanophotonics Technology Center, Universidad Politécnica de Valencia, Camino de Vera s/n, \\ Ed. IDI-5, 46022 Valencia, Spain \\ ${ }^{2}$ DAS PHOTONICS, S.L, Ciudad Politécnica de la Innovación, Camino de Vera s/n Ed. 9B, 46022 Valencia, Spain \\ *Corresponding author: jlcorral@ntc.upv.es
}

Received February 12, 2008; accepted April 23, 2008;

posted May 7, 2008 (Doc. ID 92717); published June 5, 2008

A photonic vector modulator architecture for generating pure quadrature amplitude modulation (QAM) signals is presented. An electrical quadrature-modulated signal at microwave-millimeter-wave frequencies is generated from its corresponding baseband in-phase $(I)$ and quadrature $(Q)$ components. In the proposed scheme, no electrical devices apart from the electrical tone oscillator are needed in the generation process. In addition, the purity of the generated signal is increased, and the hardware requirements are reduced when compared with previously proposed architectures so a highly compact low-cost architecture can be implemented. A pure 1.25 Gbit/s 4-QAM signal has been experimentally generated at a $42 \mathrm{GHz}$ carrier frequency. (C) 2008 Optical Society of America

OCIS codes: $060.4080,060.5625$.

The data rates required by broadband wireless systems are increasing, mainly owing to the need of high-performance voice, video, image, and data communications. This increment in data rates is pushing the development of solutions for wireless transmission of Gbit/s data rates, mainly in the millimeterwave frequency band. Millimeter-wave photonic systems have been intensively investigated as a solution that can overcome the current limitations of purely electrical architectures, owing to the inherent benefits of photonic components and optical fibers, such as huge bandwidth, scalability employing wavelength multiplexing techniques, compactness, or low transmission loss.

In recent years, the wireless transmission of data rates beyond $1 \mathrm{Gbit} / \mathrm{s}$ has been demonstrated [1,2] at millimeter-wave frequencies by employing both photonic techniques and advanced electrical circuitry. These transmissions have been realized by simply upconverting the baseband digital signal up to the desired frequency, which requires a huge electrical bandwidth in the wireless link. Then, an alternative solution is to reduce the utilized bandwidth by employing more efficient electrical modulation techniques such as quadrature amplitude modulation (QAM), as it is common in any actual digital wireless system. Photonic vector modulation (PVM) schemes have been presented to generate QAM signals by photonic processing [3,4]. However, these PVM schemes use complex electrical control circuits to translate binary data information into optical modulated signals, and the demonstrated bit rate is limited to $1 \mathrm{Mbit} / \mathrm{s}$.

Recently, we have proposed new PVM architectures with low hardware requirements, both optical and electrical [5-7]. With these PVM architectures, electrical quadrature amplitude modulations from 4-QAM up to 16-QAM and 3.6 Gbit/s data rate have been experimentally demonstrated. However, these interesting PVM schemes show an important system limitation: The generated electrical QAM signal at the photodiode output includes a high level of electrical carrier, when a real QAM signal would have no electrical carrier component at all. This undesired electrical carrier level will reduce the maximum available signal power at the output of any power amplifier, reducing at the same time the coverage area of our wireless system.

In this Letter, a modified PVM scheme is presented that generates a pure QAM signal without any undesired electrical carrier component, keeping the low hardware complexity of the original scheme.

A schematic of the proposed PVM architecture is depicted in Fig. 1. Two semiconductor lasers, emitting at wavelengths $\lambda_{1}$ and $\lambda_{2}$, and linearly biased, are directly modulated by two baseband digital signals corresponding to the modulating $x_{I}(t)$ and $x_{Q}(t)$ driving currents. A third cw laser has been added to the previously proposed PVM scheme [5]. All three optical signals are combined and externally modulated by an electrical local oscillator (LO) frequency tone in an external quadrature-biased MachZehnder modulator (MZM). Then, the signals are transmitted over a standard single-mode fiber (SSMF) optical link, whose length and dispersion are

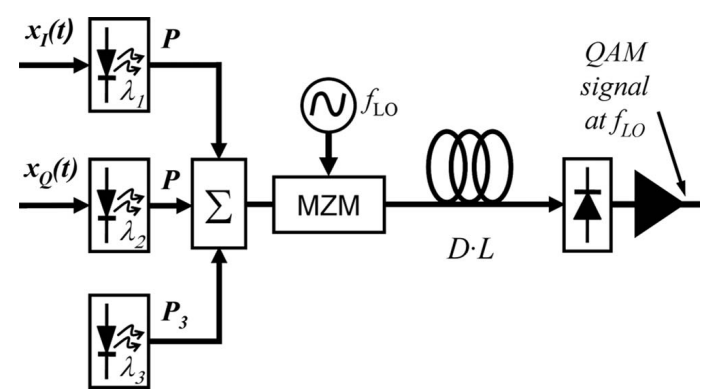

Fig. 1. PVM architecture for pure electrical QAM generation. Abbreviations are defined in text. 
$L(\mathrm{~m})$ and $D(\mathrm{ps} / \mathrm{km} \times \mathrm{nm})$ respectively, and photodetected.

The fiber's chromatic dispersion induces a delay between both $I$ - and $Q$-modulated optical signals equal to $\Delta \tau_{21}=D L \Delta \lambda_{21}$, where $\Delta \lambda_{21}=\lambda_{2}-\lambda_{1}$. This delay can be tuned by changing the fiber length $L$ or the wavelength spacing $\Delta \lambda_{21}$ in order to obtain a delay of a quarter of the electrical LO tone period (named quadrature condition), which corresponds with a differential phase of $\pi / 2$ radians between the $I$ - and $Q$-LO components. At the same time, a differential delay, $\Delta \tau_{31}=D L\left(\lambda_{3}-\lambda_{1}\right)$, is induced between the unmodulated carrier $\lambda_{3}$ and the $I$-modulated optical carrier $\lambda_{1}$. The LO component of the photocurrent for quadrature condition is given by

$$
\begin{aligned}
i_{p d-\mathrm{LO}}(t) \approx & \frac{\Re}{L_{\mathrm{opt}}} 2 \mathrm{~J}_{1}\left(m_{\mathrm{LO}}\right) \mathrm{J}_{0}\left(m_{\mathrm{LO}}\right)\left\{P _ { 3 } \operatorname { c o s } \left(\omega_{\mathrm{LO}} t\right.\right. \\
& \left.-\frac{\Delta \tau_{31}}{\Delta \tau_{21}} \frac{\pi}{2}\right)+P \sqrt{2} \cos \left(\omega_{\mathrm{LO}} t-\frac{\pi}{4}\right) \\
& +\eta\left[x_{I}(t) \cos \left(\omega_{\mathrm{LO}} t\right)\right. \\
& \left.\left.+x_{Q}(t) \cos \left(\omega_{\mathrm{LO}} t-\frac{\pi}{2}\right)\right]\right\}
\end{aligned}
$$

where $\omega_{\mathrm{LO}}$ is the LO angular frequency; $\mathfrak{R}$ is the photodiode responsivity; $L_{\text {opt }}$ are the optical losses from laser output to photodiode input; $P$ is the mean optical power emitted by each of the optical sources $\lambda_{1}$ and $\lambda_{2} ; P_{3}$ is the optical power emitted by the optical source $\lambda_{3} ; \eta$ is the slope efficiency of the laser diodes in W/A; $J_{0}$ and $J_{1}$ are the zeroth and first order Bessel functions of the first kind, respectively; and $m_{\mathrm{LO}}=\pi V_{\mathrm{LO}} / V_{\pi}$, with $V_{\mathrm{LO}}$ being the amplitude of the LO tone and $V_{\pi}$ being the MZM half-wave voltage.

From Eq. (1) it can be observed that data signals are quadrature modulated over the LO carrier, since a $\pi / 2$ radians phase difference in the cosine argument is induced by the fiber chromatic dispersion. Moreover, if the wavelength of the third laser is selected to fulfill the ratio $\Delta \tau_{31}=2.5 \Delta \tau_{21}$ and its power to be $P_{3}=(P)^{1 / 2}$, then the two first terms of Eq. (1) will cancel each other, and a pure QAM signal at $\omega_{\mathrm{LO}}$ will be obtained at the photodiode output.

The PVM scheme shown in Fig. 1 was employed to validate the proposed approach. A set of three distributed-feedback lasers (wavelengths $\lambda_{1}$ $=1550.92 \mathrm{~nm}, \quad \lambda_{2}=1549.026 \mathrm{~nm}, \quad$ and $\quad \lambda_{3}$ $=1547.356 \mathrm{~nm}$ ) with $1 \mathrm{GHz}$ analog modulation bandwidth were used in order to generate a $1.25 \mathrm{Gbit} / \mathrm{s}$ 4-QAM signal at the LO frequency of $42 \mathrm{GHz}$. Only two lasers $\left(\lambda_{1}, \lambda_{2}\right)$ were directly modulated by two $0.625 \mathrm{Gbit} / \mathrm{s}$ nonreturn to zero signals with $2 \mathrm{~V}$ amplitude peak to peak. In all cases, the optical signals from the lasers were polarization controlled and combined in a coupling matrix composed by two cascaded $3 \mathrm{~dB}$ optical couplers. The corresponding optical signals were modulated by $\mathrm{a}+15 \mathrm{dBm}$ LO tone at
$42 \mathrm{GHz}$ in a $50 \mathrm{GHz}$ bandwidth MZM biased at the quadrature point. After the external modulation process, the optical signals are transmitted over a $340 \mathrm{~m}$ $\mathrm{SSMF}$ coil $(D=16.5 \mathrm{ps} / \mathrm{km} \times \mathrm{nm})$ in order to accomplish the quadrature condition $\Delta \tau_{21}=1 /\left(f_{\mathrm{LO}} * 4\right)$.

The electrical spectrum of the generated signals, measured with an electrical spectrum analyzer, is shown in Fig. 2 for the cases in which the unmodulated laser is on [Fig. 2(a)] or off [Fig 2(b)]. When the $\mathrm{cw}$ laser is off, the undesired LO carrier level can be clearly observed [Fig. 2(a)]. On the other hand, when the cw laser was set active with an optical power level and wavelength according to previously stated calculations, the undesired LO component is totally suppressed with a suppression ratio higher than $30 \mathrm{~dB}$, as observed in Fig. 2(b).

To measure the quality of the optically generated QAM signal, it was downconverted to baseband by mixing it in an electrical mixer with a phase-adjusted replica of the LO tone used in the generation process as it is depicted in Fig. 3. By means of an electrical delay line (EDL), the electrical phase of the LO replica may be adjusted in order to independently downconvert the $I$ and $Q$ digital signals in what would confirm the fulfillment of the quadrature condition. The eye diagrams for these demodulated baseband digital signals were measured using a digital communications analyzer (DCA).
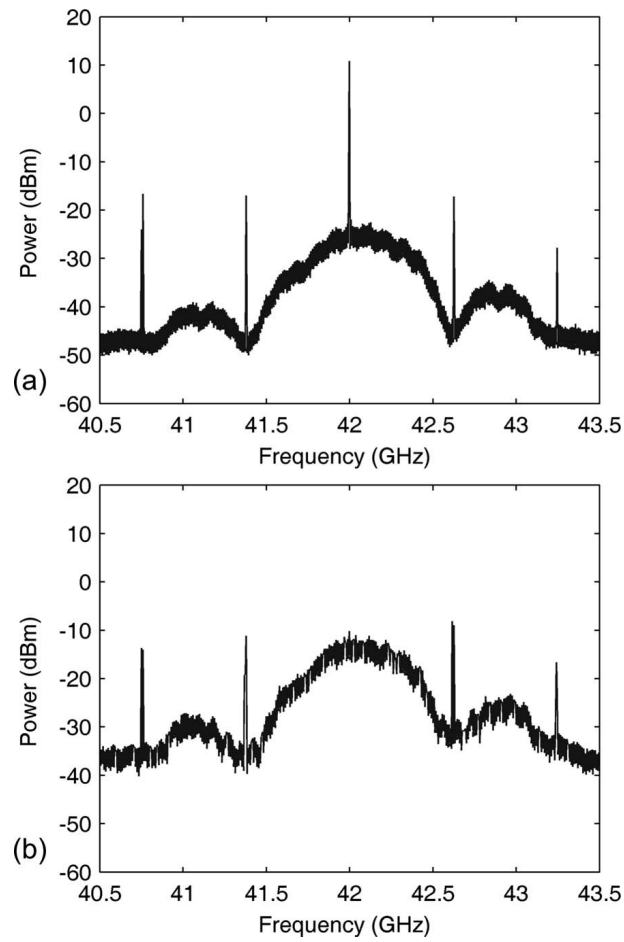

Fig. 2. Electrical spectrum at the photodiode output in the PVM scheme from Fig. 1 when the optical source $\lambda_{3}$ is (a) off and (b) on, corresponding to a $1.25 \mathrm{~Gb} / \mathrm{s} 4-\mathrm{QAM}$ signal at a $42 \mathrm{GHz}$ electrical carrier. 


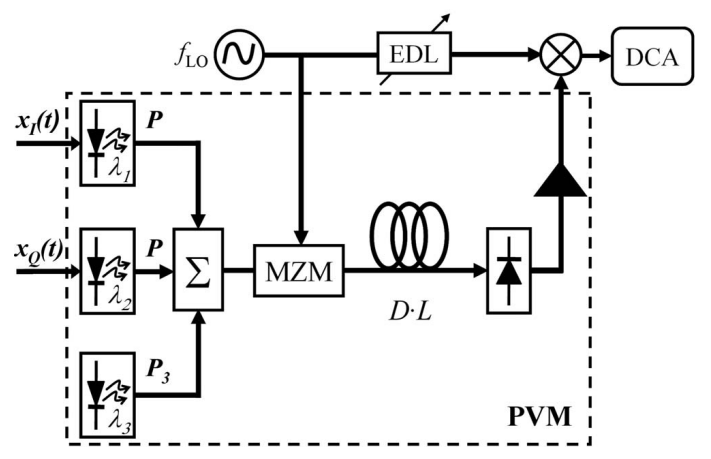

Fig. 3. Measurement setup to electrically analyze the quality of the QAM modulation generated by the PVM. Abbreviations are defined in text.

The measured eye diagrams are shown in Fig. 4 where a full open eye can be clearly identified for both $I$ and $Q$ digital streams. The $Q$ factors of the signals measured by the DCA were $Q=8.5$ and $Q=7.7$, which correspond to bit-error-rate (BER) values equal to $9 \mathrm{e}-18$ and $7 \mathrm{e}-15$, respectively. These excel-

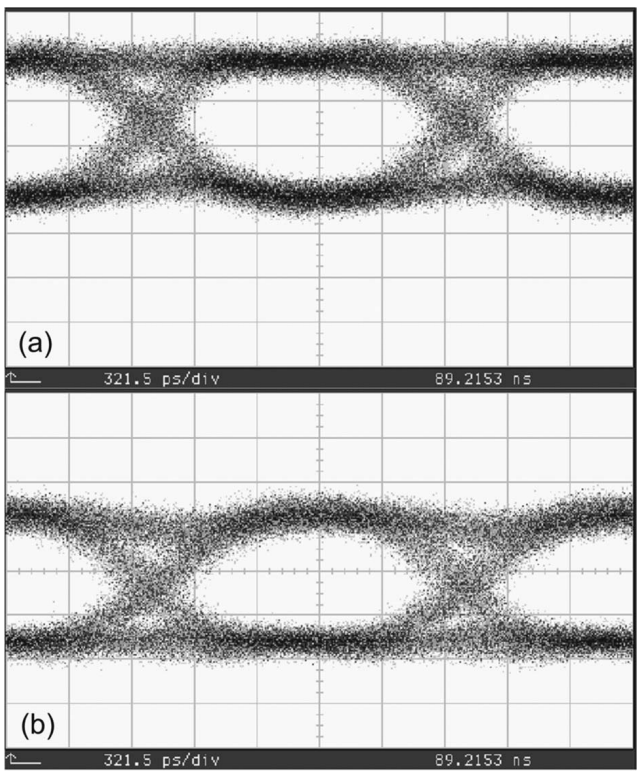

Fig. 4. Eye diagram for the $0.625 \mathrm{Gbit} / \mathrm{s}$ data signals corresponding to the electrically demodulated (a) in-phase and (b) quadrature components of the $1.25 \mathrm{Gbit} / \mathrm{s} 4-\mathrm{QAM}$ signal generated at $42 \mathrm{GHz}$ with the PVM shown in Fig. 1. lent BER values prove that the proposed PVM architecture is able to generate a high-quality QAM signal that after electrical demodulation offers a practically error free back-to-back transmission.

In this work, a photonic vector modulator architecture for generating pure electrical QAM signals has been shown. This architecture offers a low optical hardware complexity combined with no additional electrical components but the local oscillator to generate the electrical carrier and the digital streams corresponding to the in-phase and quadrature components. The proposed PVM scheme is a modification of PVM architectures previously proposed by the authors where an additional cw laser has been added in order to generate a pure QAM signal, keeping at the same time the good performance of the previous PVM architecture. Even though the insertion of an extra laser would increase the total laser noise of the system, the quality of the generated QAM signal at the photodetector output has not been affected, as the practically error-free measurements results have shown in a back-to-back $1.25 \mathrm{Gbit} / \mathrm{s}$ transmission at $42 \mathrm{GHz}$ with photonic generation and electrical detection. The quadrature condition of the photonically generated QAM signal has been checked by independently selecting any of the two digital streams ( $I$ and $Q)$ just by acting on the electrical phase of the local oscillator used in the electrical receiver.

This work has been supported by the European Commission FP6 under project IST-35317 IPHOBAC. Financial support by the Spanish Ministry of Education and Science and EU-FEDER (European UnionEuropean Regional Development Fund) under the project TEC2005-08298-C02-02 and the Valencia Regional Government under the project ACOMP2007/ 144 is gratefully acknowledged.

\section{References}

1. A. Hirata, M. Harada, and T. Nagatsuma, J. Lightwave Technol. 21, 2145 (2003).

2. A. Wiberg, P. Perez-Milan, M. V. Andres, P. A. Andrekson, and P. O. Hedekvist, IEEE Photon. Technol. Lett. 17, 1938 (2005).

3. W. D. Jemison, A. J. Kreuzberger, and E. Funk, IEEE Microw. Wirel. Compon. Lett. 12, 125 (2002).

4. P. Candelas, J. M. Fuster, J. Martí, and J. C. Roig, J. Lightwave Technol. 21, 496 (2003).

5. M. A. Piqueras, B. Vidal, V. Polo, J. L. Corral, and J. Martí, IEEE Photon. Technol. Lett. 17, 1947 (2005).

6. R. Sambaraju, M. A. Piqueras, V. Polo, J. L. Corral, and J. Marti, J. Lightwave Technol. 25, 3350 (2007).

7. R. Sambaraju, M. A. Piqueras, V. Polo, J. L. Corral, and J. Marti, in Proceedings of European Conference on Optical Communications 2007 (VDE Verlag, 2007), paper Tu5.4.7. 


\title{
Paper E
}

\section{Pure 2.5 Gb/s 16-QAM Signal Generation with Photonic Vector Modulator}

\author{
J. L. Corral, R. Sambaraju, M. A. Piqueras, and V. Polo
}

Presented at IEEE MTT-S International Microwave Symposium Digest, MTT-S IMS 2008, pages 595-598, Atlanta GA, June 2008. 


\title{
Pure $2.5 \mathrm{~Gb} / \mathrm{s}$ 16-QAM Signal Generation with Photonic Vector Modulator
}

\author{
J. L. Corral', R. Sambaraju', M.A. Piqueras ${ }^{2}$, V. Polo ${ }^{1}$ \\ ${ }^{1}$ Valencia Nanophotonics Technology Center, Univ. Politécnica de Valencia \\ Camino de Vera s/n, Ed. IDI-5, 46022 Valencia (SPAIN) \\ ${ }^{2}$ DAS PHOTONICS, S.L, Ciudad Politécnica de la Innovación \\ Camino de Vera s/n Ed. 9B, 46022 Valencia (SPAIN)
}

\begin{abstract}
A novel photonic vector modulator architecture for generating pure quadrature amplitude modulation (QAM) signals is presented. No electrical devices apart from the local oscillator are needed in the generation process. Both binary and multilevel digital signals can be used to generate quadrature phase shift keying (QPSK) or multilevel QAM (M-QAM) digital modulations at any carrier frequency. A pure $2.5 \mathrm{~Gb} / \mathrm{s} 16-\mathrm{QAM}$ signal has been experimentally generated at a $42 \mathrm{GHz}$ carrier frequency.
\end{abstract}

Index Terms - Optical modulation, Optical signal processing, Quadrature amplitude modulation, Radio communication,.

\section{INTRODUCTION}

The data rates required by broadband wireless systems are increasing, mainly due to the need of high performance voice, video, image and data communications. This increment in data rates is pushing the development of solutions for wireless transmission of $\mathrm{Gb} / \mathrm{s}$ data rates, mainly in the millimeter wave frequency band. Millimeter wave photonic systems have been intensively investigated as a solution that can overcome the current limitations of purely electrical architectures, due to the inherent benefits of photonic components and optical fibers, such as huge bandwidth, scalability employing wavelength multiplexing techniques, compactness, or low transmission loss.

In recent years, the wireless transmission of data rates beyond $1 \mathrm{~Gb} / \mathrm{s}$ has been demonstrated [1,2] at millimeter wave frequencies by employing both photonic techniques and advanced electrical circuitry. These transmissions have been realized by simply up-converting the base band digital signal up to the desired frequency which requires a huge electrical bandwidth in the wireless link. Then, an alternative solution is to reduce the utilized bandwidth by employing more efficient electrical modulation techniques such as quadrature amplitude modulation (QAM) as it is common in any actual digital wireless system. Photonic vector modulation (PVM) schemes have been presented to generate QAM signals by photonic processing $[3,4]$. However, these PVM schemes use complex electrical control circuit to translate binary data information into optical modulated signals and the demonstrated bit rate was limited to $1 \mathrm{Mb} / \mathrm{s}$.
Recently, we have proposed new PVM architectures with low hardware, both optical and electrical, requirements [5-7]. With these PVM architectures, electrical quadrature amplitude modulations from 4-QAM up to 16-QAM and $3.6 \mathrm{~Gb} / \mathrm{s}$ data rate has been experimentally demonstrated. However, these interesting PVM schemes show an important system limitation, the electrical QAM generated signal at the photodiode output includes a high level of electrical carrier when a real QAM signal would have no electrical carrier component at all. This undesired electrical carrier level will reduce the available electrical signal power level at the output of any power amplifier stage, reducing at the same time the coverage area of our wireless system.

In this paper, a modified PVM scheme is presented which generates a pure QAM signal without any undesired electrical carrier component and keeping the low hardware complexity of the original PVM scheme. Besides, this new PVM scheme is able to generate any M-QAM digital modulation without any modification in the PVM configuration, thus, being able to accommodate both binary and multilevel digital signals as electrical inputs to the photonic vector modulation process.

\section{PHOTONIC VECTOR MODULATOR ARCHITECTURE DESCRIPTION}

A schematic of the proposed PVM architecture is depicted in Fig. 1. Two semiconductor lasers, emitting at wavelengths $\lambda_{1}$ and $\lambda_{2}$, and linearly biased are directly modulated by two base band digital signals corresponding to the modulating $x_{I}(t)$ and $x_{Q}(t)$ driving currents. In order to generate a 4-QAM digital modulation both $x_{l}(t)$ and $x_{Q}(t)$ would correspond to binary digital signals. However, if a 16-QAM modulation is desired both $x_{t}(t)$ and $x_{O}(t)$ will correspond to 4-level digital streams. A third continuous wave $(\mathrm{CW})$ laser has been added to the previously proposed PVM scheme [5].

All three optical signals are combined and externally modulated by an electrical local oscillator (LO) frequency tone in an external quadrature biased Mach-Zehnder modulator (MZM). Then, the signals are transmitted over a standard single-mode fiber (SSMF) optical link, whose length and dispersion are $L(\mathrm{~m})$ and $D(\mathrm{ps} / \mathrm{km} \cdot \mathrm{nm})$ respectively, and finally photodetected. 


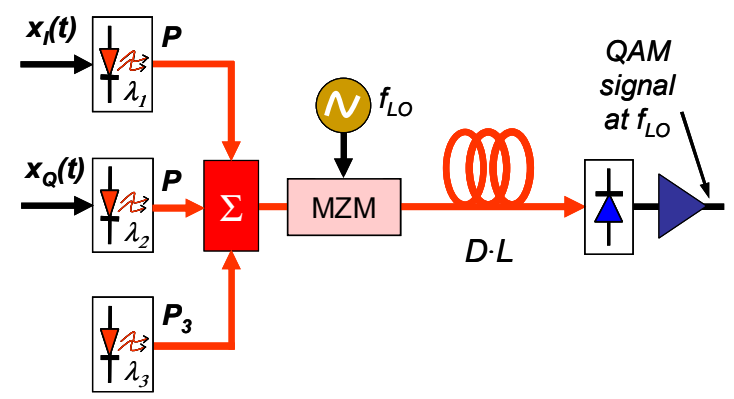

Fig. 1. Photonic Vector Modulator architecture for pure QAM generation.

The optical fiber chromatic dispersion induces a delay between both I- and Q-modulated optical signals equal to $\Delta \tau_{21}=D \cdot L \cdot \Delta \lambda_{21}$, where $\Delta \lambda_{21}=\lambda_{2}-\lambda_{1}$. This delay can be tuned by changing the fiber length or the wavelength spacing $\Delta \lambda_{21}$ in order to obtain a delay of a quarter of the electrical LO tone period (named quadrature condition), which corresponds with a differential phase of $\pi / 2$ radians between the I- and Q-LO components. At the same time, a differential delay $\Delta \tau_{31}=D \cdot L \cdot\left(\lambda_{3}-\lambda_{1}\right)$, is induced between the unmodulated carrier $\lambda_{3}$ and the I-modulated optical carrier $\lambda_{1}$. When all these conditions are fulfilled, the LO component of the detected photocurrent would be:

$$
\begin{aligned}
& i_{p d-L O}(t) \approx \frac{\Re}{L_{o}} 2 J_{1}\left(m_{L O}\right) J_{0}\left(m_{L O}\right) . \\
& {\left[P_{3} \cos \left(\omega_{L O} t-\frac{\Delta \tau_{31}}{\Delta \tau_{21}} \frac{\pi}{2}\right)+P \sqrt{2} \cos \left(\omega_{L O} t-\frac{\pi}{4}\right) \cdot(1)\right.} \\
& \left.+\eta\left[x_{I}(t) \cos \left(\omega_{L O} t\right)+x_{Q}(t) \cos \left(\omega_{L O} t-\frac{\pi}{2}\right)\right]\right]
\end{aligned}
$$

where $\omega_{L O}$ is the LO angular frequency, $\Re$ is the photodiode responsivity in $\mathrm{A} / \mathrm{W}, L_{o}$ are the optical losses from any laser output to the photodiode input, $P$ is the mean optical power emitted by each of the optical sources $\lambda_{1}$ and $\lambda_{2}, P_{3}$ is the optical power emitted by the optical source $\lambda_{3}, \eta$ is the slope efficiency of the laser diodes in W/A, $J_{0}$ and $J_{l}$ are the zero-th and first order Bessel functions of the first kind, respectively, and $m_{L O}=\pi V_{L O} / V_{\pi}, V_{L O}$ being the amplitude of the local oscillator tone and $V_{\pi}$ being the MZM half-wave voltage.

From (1) it can be observed that data signals are quadrature modulated over the LO carrier since a $\pi / 2$ radians phase difference in the cosine argument is induced by the fiber chromatic dispersion. Moreover, if the wavelength of the third laser is selected to fulfill the ratio $\Delta \tau_{31}=2.5 \Delta \tau_{21}$ and its power to be $P_{3}=P \cdot(2)^{1 / 2}$ then the two first terms of (1) will cancel each other and a pure QAM signal at $\omega_{L O}$ will be obtained at the photodiode output.
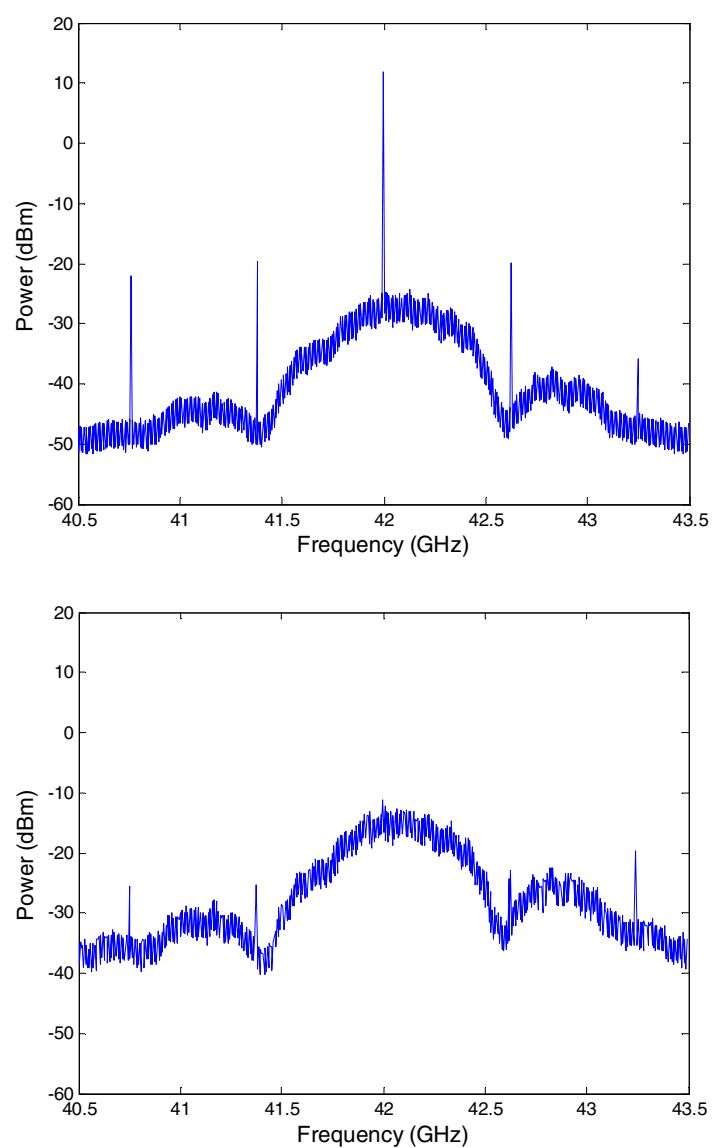

Fig. 2. Electrical spectrum at the photodiode output in the PVM scheme from Fig. 1 when the optical source $\lambda_{3}$ is OFF (above) and $\mathrm{ON}$ (below) corresponding to a $2.5 \mathrm{~Gb} / \mathrm{s} 16-\mathrm{QAM}$ signal at $42 \mathrm{GHz}$ electrical carrier.

\section{EXPERIMENTAL RESULTS}

The PVM scheme shown in Fig. 1 was employed to validate the proposed approach. A set of three DFB lasers (wavelengths, $\quad \lambda_{1}=1550.92 \mathrm{~nm}, \quad \lambda_{2}=1549.026 \mathrm{~nm}$ and $\lambda_{2}=1547.356 \mathrm{~nm}$ ) with $1 \mathrm{GHz}$ analog modulation bandwidth were used in order to generate a $2.5 \mathrm{~Gb} / \mathrm{s} 16-\mathrm{QAM}$ signal at a LO frequency equal to $42 \mathrm{GHz}$. Two $625 \mathrm{Mbaud} \mathrm{NRZ}$ (non-return-to-zero) digital streams with four amplitude level each were generated by means of an arbitrary waveform generator (AWG). Only two lasers $\left(\lambda_{1}, \lambda_{2}\right)$ were directly modulated by the $1.25 \mathrm{~Gb} / \mathrm{s}$ 4-levels digital signals with $2 \mathrm{~V}$ 
amplitude peak to peak. In all cases, the optical signals were polarization controlled and combined in a coupling matrix composed by two cascaded $3 \mathrm{~dB}$ optical couplers. All three optical signals were externally modulated by a $+15 \mathrm{dBm}$ LO tone at $42 \mathrm{GHz}$ in a $50 \mathrm{GHz}$ bandwidth MZM biased at the quadrature point. After the external modulation process, the optical signals are transmitted over a 340 meters of SSMF $(\mathrm{D}=16.5 \mathrm{ps} / \mathrm{km} \cdot \mathrm{nm})$ in order to fulfill the dispersion induced phase conditions defined in Section II and photodetected.

The electrical spectra of the generated signals, measured with an electrical spectrum analyzer are shown in Fig. 2 for the cases in which the unmodulated laser is ON (right) or OFF (left). When the CW laser is OFF, the undesired LO carrier level can be clearly observed (Fig. 2 left). On the other hand, when the third laser was set active with an optical power level and wavelength according to Section II calculations, the undesired LO component is totally suppressed with a suppression ratio higher than $40 \mathrm{~dB}$, as can be seen in Fig. 2 by comparing both spectra.

In order to measure the quality of the optically generated QAM signal, it was down converted to base band by mixing it in an electrical mixer with a phase adjusted replica of the LO tone used in the generation process as it is depicted in Fig. 3. The eye-diagrams for these demodulated baseband digital signals were measured using a digital communications analyzer (DCA). By changing the electrical phase of the LO replica, both I and Q digital signals were independently down converted confirming that the quadrature condition was obtained.

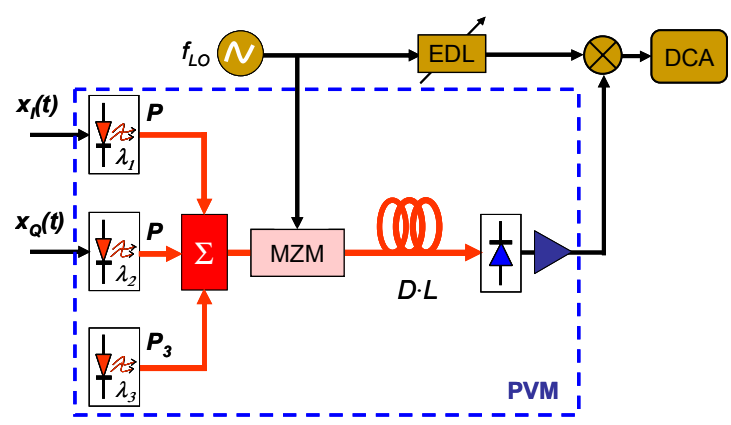

Fig. 3. Measurement setup to electrically analyze the quality of the QAM modulation generated by the PVM.

The measured eye diagrams are shown in Fig. 4 where the three eyes corresponding to the 4 amplitude levels of the digital signals are clearly identified. The relatively noisy eye diagrams form Fig. 4 are affected by the electrical components used to down convert the optically generated QAM signal to be measured by the DCA where no external electrical filter were used to reduce the electrical noise entering the equipment. Thus, the actual quality of the generated QAM signal is better than this value.
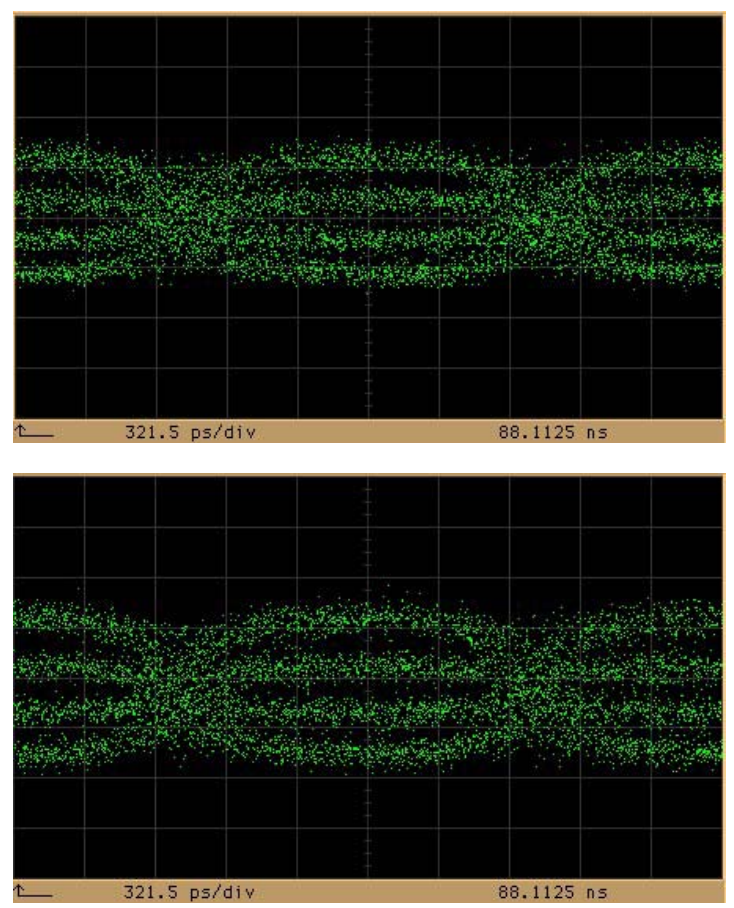

Fig. 4. Eye diagrams for the $1.25 \mathrm{~Gb} / \mathrm{s}$ data signals corresponding to the electrically demodulated in phase (above) and quadrature (below) components of the $2.5 \mathrm{~Gb} / \mathrm{s} 16-\mathrm{QAM}$ signal generated at $42 \mathrm{GHz}$ with the PVM shown in Fig. 1.

From the eye diagrams shown in Fig. 4, the signal constellation has been extracted by sampling at the center of the symbol period. The signal constellation is shown in Fig. 5 were the 16 symbols of the 16-QAM are clearly identified. From this constellation the EVM (Error Vector Magnitude) has been calculated and a value of $-17.05 \mathrm{~dB}$ has been obtained. The constellation points from Fig. 5 are not equally spaced due to some compression at the driving conditions of the modulated lasers. This situation could be solved if more linear driver amplifiera and lasers with a broader modulation range were available for the measurements.

In order to check the influence of the insertion of the CW laser in the PVM scheme from Fig. 1 the same measurements were carried out in the configuration with only two lasers active. In this case the EVM was reduced to $-17.85 \mathrm{~dB}$ which implies an increment in SNR (signal-to-noise ratio) of $0.8 \mathrm{~dB}$. Thus, a $0.8 \mathrm{~dB}$ penalty in SNR is induced by the insertion of an unmodulated laser to eliminate the undesired LO carrier leakage at the PVM output. 


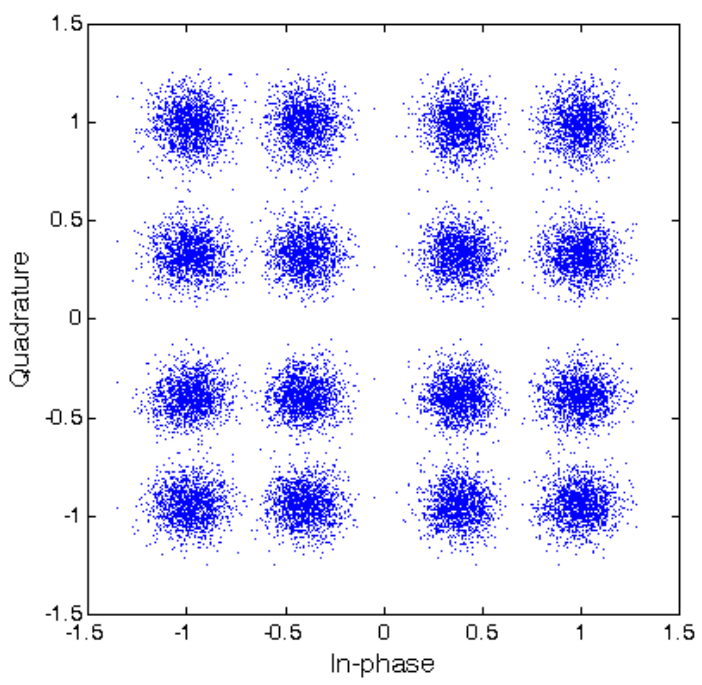

Fig. 5. 16-QAM signal constellation extracted from the electrically detected digital signals of the $2.5 \mathrm{~Gb} / \mathrm{s}$ 16-QAM signal optically generated at $42 \mathrm{GHz}$ with the PVM shown in Fig. 1.

\section{REMARKS AND CONCLUSION}

In this work, a new photonic vector modulator architecture has been shown. This architecture offers a low optical hardware complexity combined with no additional electrical components but the local oscillator to generate the electrical carrier and the digital streams corresponding to the in-phase and quadrature components.

The proposed PVM scheme is a modification of PVM architectures previously proposed by the authors with the addition of an additional $\mathrm{CW}$ laser in order to generate a pure QAM signal, keeping at the same time the good performance of the previous PVM architecture. At the same time, it has been proved that this PVM scheme is able to generate MQAM modulation by driving the laser with multilevel digital streams. The additional laser noise due to the additional laser is translated to an $0.8 \mathrm{~dB}$ penalty in the signal-to-noise ratio at the PVM output. Using this novel PVM technique, the generation of multi-Gb/s multilevel quadrature amplitude modulations (M-QAM) at mm-wave carriers is feasible. As a proof of concept a pure $2.5 \mathrm{~Gb} / \mathrm{s}$ 16-QAM signal at $42 \mathrm{GHz}$ carrier has been experimentally generated.

\section{ACKNOWLEDGEMENT}

This work has been supported by the European Commission FP6 under project IST-35317 IPHOBAC. Financial support by the Spanish MCyT and EU-FEDER under the project TEC2005-08298-C02-02 and the Valencia Regional Government under the project ACOMP2007/144 is gratefully acknowledged.

\section{REFERENCES}

[1] A. Hirata M. Harada, T. Nagatsuma, "120-GHz wireless link using photonic techniques for generation, modulation, and emission of millimeter-wave signals", IEEE/OSA Journal of Lightwave Technology, vol. 21, no. 10, pp. 2145-2153, October 2003.

[2] A. Wiberg, P. Perez-Milan, M. V. Andres, P. A. Andrekson, P. O. Hedekvist, "Fiber-Optic 40-GHz mm-Wave Link With 2.5Gb/s Data Transmission", IEEE Photonics Technology Letters, Vol. 17, No. 9, pp. 1938-1940, September 2005.

[3] W. D. Jemison, A. J. Kreuzberger, and E. Funk, "Microwave photonic vector modulator for high-speed wireless digital communications," IEEE Microw. Compon. Lett., vol. 12, no. 4, pp. 125-127, April 2002.

[4] P. Candelas, J. M. Fuster, J. Martí, and J. C. Roig, "Optically generated electrical-modulation formats in digital-microwave link applications," IEEE/OSA Journal of Lightwave. Technology, vol. 21, no. 2, pp. 496-499, February 2003.

[5] M.A. Piqueras, B. Vidal, V. Polo, J. L. Corral, J. Martí, "Direct Photonic Generation of Electrical Vector Modulations at Microwave/Millimeter-wave Frequencies", IEEE Photonics Technology Letters, vol. 17, no. 9, pp. 1947-1949, September 2005.

[6] R. Sambaraju, M. A. Piqueras, V. Polo, J. L. Corral, J. Marti, "Generation of Multi-Gbps MQAM/MPSK Modulated MmWave Carriers Employing Photonic Vector Modulator Techniques", IEEE/OSA Journal of Lightwave Technology, vol. 25, no.11, pp. 3350-3357, November 2007.

[7] R. Sambaraju, M. A. Piqueras, V. Polo, J. L. Corral, J. Marti, "Generation of $3.6 \mathrm{Gbps}$ 16-QAM $39 \mathrm{GHz} \mathrm{mm}$-wave carrier employing photonic vector modulation", 2007 European Conference on Optical Communications Digest, Tu5.4.7, September 2007. 



\title{
Paper F
}

\section{Photonic Vector Modulation of 3.6 Gb/s 16-QAM at $39 \mathrm{GHz}$ for Radio-on-Fiber Systems}

\author{
R. Sambaraju, M. A. Piqueras, V.Polo, J. L. Corral and J. Marti
}

Presented at 32nd European Conference on Optical Communications, ECOC 2007, paper Tu 5.4.7, Berlin Germany, Sept 2007. 


\title{
Photonic Vector Modulation of 3.6 Gb/s 16QAM at 39 GHz for Radio-on- Fibre Systems.
}

\author{
R. Sambaraju (1), M. A. Piqueras (2), V. Polo (1), J. L. Corral (1), J. Martí (1) \\ 1: Nanophotonics Technology Center, Universidad Politécnica de Valencia, Camino de Vera s/n, Ed. IDI-5, \\ Acceso A, Nivel 1, 46022 Valencia, Spain. E-mail: vpolor@ntc.upv.es \\ 2: DAS Photonics, S.L., Ciudad Politécnica de la Innovación, Camino de Vera s/n Ed. 9B, 46022, Valencia, \\ Spain. E-mail: mapiqueras@dasphotonics.com.
}

\begin{abstract}
The experimental generation of a 3.6Gb/s-16QAM 39GHz carrier employing Photonic Vector Modulation is reported for the first time. The quadrature condition is introduced using an optical delay line. EVM is estimated from measured eye-diagrams.
\end{abstract}

\section{Introduction}

The increasing capacity demand of wireline and wireless access networks is pushing the development of solutions for wireless transmission of $\mathrm{Gb} / \mathrm{s}$ data rates, mainly in the mm-wave frequency band. Millimeter wave photonic systems have been intensively investigated as a solution that can overcome the current limitations of purely electrical architectures, due to the inherent benefits of photonic components and optical fibres, such as huge bandwidth, scalability employing wavelength multiplexing techniques, compactness, or low transmission loss [1-5]. Due to the high data rates envisaged, techniques to reduce the occupied spectrum are desireble, for instance using multi-level modulation formats [6-8].

Photonic vector modulation (PVM) of baseband data is one of the most promising techniques as allows the generation of the multi-level signals directly at the $\mathrm{mm}$-wave carrier frequency and the remote delivery of the modulated carrier and baseband data. The generation of $3 \mathrm{~Gb} / \mathrm{s}$ QPSK and 8QAM signals at $39 \mathrm{GHz}$ has been previously demonstrated using PVM $[9,10]$.

In this paper we present the generation of a 3.6 $\mathrm{Gb} / \mathrm{s} 16$ level quadrature amplitude modulated 39 $\mathrm{GHz} \mathrm{mm}$-wave carrier employing directly modulated lasers (data) and external modulators (mm-wave carrier). The quadrature condition between the inphase and the quadradure components of baseband data is achieved using an tunable optical delay line (ODL), with the advantage of fiber transmission induced delay compensation. Error vector magnitude (EVM) of the 16 QAM signal is estimated using the statistical data of the demodulated signals.

\section{Operating principle and experimental set-up}

Four different baseband data $\left(I_{1}, I_{2}, Q_{1}, Q_{2}\right)$ streams are used to directly modulate four lasers of different wavelengths. Different wavelengths are chosen to avoid coherent phase noise after photo detection. I1 and $\mathrm{I}_{2}$ are combined resulting in a four level baseband ASK signal, where $I_{2}$ is attenuated by $3 \mathrm{~dB}$. Similarly $Q_{1}$ and $Q_{2}$ are combined. The outputs of the two $3 \mathrm{~dB}$ couplers are the in-phase (I) and quadrature phase (Q) 4 ASK signals as shown in figure 1 .

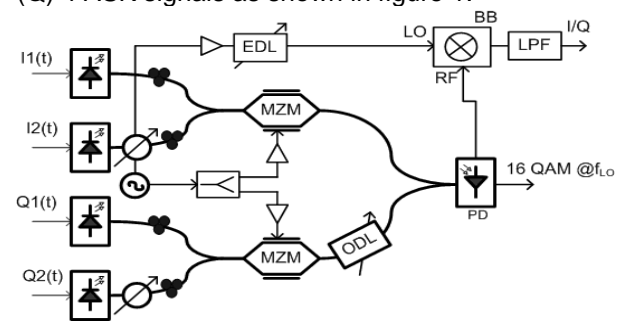

Figure 1: The schematic of the 16QAM PVM system. The RF carrier is modulated on the $\mathrm{I}$ and $\mathrm{Q}$ components using two Mach-Zehnder modulators (MZM) biased at the quadrature point. Then a tunable electrical delay line (EDL) is used to induce a $90^{\circ}$ phase shift between the I and $Q$ components by setting $\Delta t=f_{L O} / 4$, where $f_{L O}$ is the carrier frequency.

\section{Mesurements Description}

Four DFB lasers of $1 \mathrm{GHz} 3 \mathrm{~dB}$ bandwidth are used to modulate four PRBS sequences of $900 \mathrm{Mb} / \mathrm{s}$ each. The four lasers denoted by $l_{1}, l_{2}, Q_{1}$ and $Q_{2}$ are lasing at $1551.53 \mathrm{~nm}, 1549.32 \mathrm{~nm}, 1550.1 \mathrm{~nm}$ and 1547.23 $\mathrm{nm}$, respectively. Low pass filters with a cut-off frequency of $1.65 \mathrm{GHz}$ were used to filter noise before direct modulation of lasers. Laser $I_{1}$ and $I_{2}$ are mixed using a 3-dB coupler, and electrical delay line was used in one of the arms to synchronise the pulses. The same procedure was used for the Q1 and Q2 components.

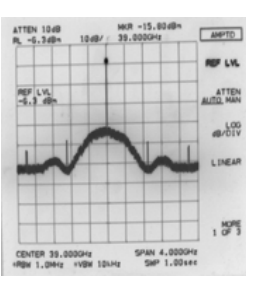

(a)

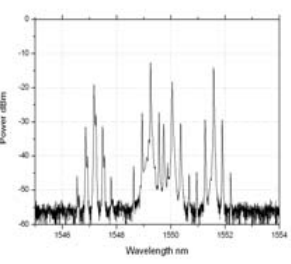

(b)
Figure 2: Spectrum of 16QAM signal at $39 \mathrm{GHz}$ : Scale X: $400 \mathrm{MHz} / \mathrm{div}$ Y: $10 \mathrm{~dB} / \mathrm{div}$ (a), Optical Spectrum (b) 
Two MZM were used to modulate the $39 \mathrm{GHz}$ carrier. The carrier was amplified to $+15 \mathrm{dBm}$ before modulation. The $Q$ component was delayed by $1 / 4 f_{L O}$ using an EDL, where $f_{L O}$ is the local oscillator frequency, to induce a $90^{\circ}$ phase shift between the I and $Q$ components, which were next added using another 3-dB coupler resulting in $3.6 \mathrm{~Gb} / \mathrm{s} 16 \mathrm{QAM}$ at $39 \mathrm{GHz}$ after photodetection. Figure 2 shows the optical spectrum and the electrical spectrum of the 16 QAM signal.

The QAM signal was demodulated by mixing with the same LO carrier amplified to $+7 \mathrm{dBm}$. Another EDL was used with the LO before mixing, to synchronise and induce the $90^{\circ}$ phase shift for detecting the I and the $Q$ components. A baseband amplifier and a low pass filter with cut-off frequency of $1 \mathrm{GHz}$ were used before estimating the EVM. Figure 3 shows the downconverted signals.

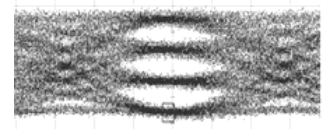

(a)

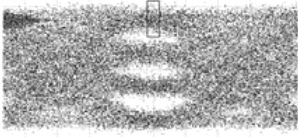

(c)

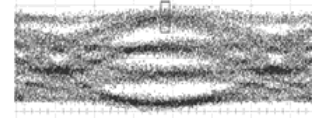

(b)

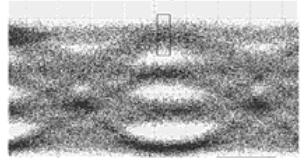

(d)
Figure 3: Eye diagrams for 4ASK back to back quadrature (a), inphase (b), demodulated quadrature (c), inphase (d).

\section{Results}

The four electrical baseband data were amplified to $1.8 \mathrm{~V} p-p$, and used to directly modulate the lasers. To determine the quality of the signal EVM is measured by measuring the mean $(\mu)$, and standard deviation $(\sigma)$ from the eye diagram. The downconverted I and $Q$ components eye diagrams were captured using an oscilloscope, and the histogram of each level was plotted and the $\mu$ and $\sigma$ were extracted. These values were later used to generate a Gaussian distribution, and calculate the EVM as

$$
E V M=\sqrt{\frac{1}{M N} \sum_{j=1}^{N} \frac{\sigma_{I, j}{ }^{2}+\sigma_{Q, j}{ }^{2}}{\mu_{I, j}^{2}+\mu_{Q, j}{ }^{2}}}
$$

Where $M$ is 16 (16QAM), and $N$ is the number of samples captured by the oscilloscope. The constellation diagram of the downconverted 16QAM signal is shown in Figure 4. The EVM of the 16 QAM signal was calculated as $-18.33 \mathrm{~dB}$, using 1200 samples of the scope. It can be seen that the I component is more degraded than the $\mathrm{Q}$ component; this is because of lack of availability of an appropriate EDL to synchronise the bits of the multilevel signal. Another issue is the interference of higher order sidebands of lasers $I_{2}$ (1549.32) and $Q_{1}$ (1550.1).

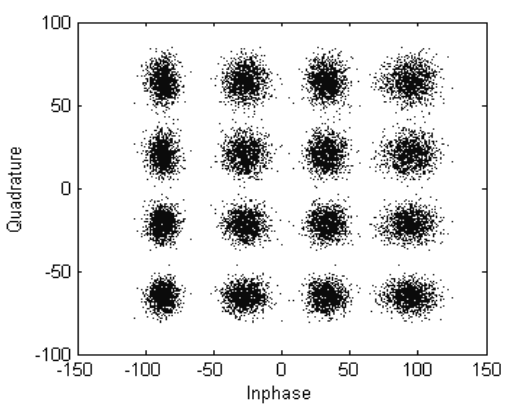

Figure 4: Constellation of the 16QAM signal.

\section{Conclusions}

The generation of $3.6 \mathrm{~Gb} / \mathrm{s}$ 16-QAM $39 \mathrm{GHz}$ signal employing a PVM technique is demonstrated for the first time to the authors knowledge. The PVM arrangement is based in direct modulation of the I and $\mathrm{Q}$ components and external modulation of the $\mathrm{mm}$ wave carrier. Hardware limitations impaired the generated signal quality but can easily overcome, e.g. by using bandwidth matched filters and additional EDL. Finally, although not shown in this paper, this scheme is suitable for remote antenna applications by using dispersion management in the fibre span by tuning the ODL shown in Fig. 1, as reported [11].

\section{Acknowledgements}

This work has been carried out within EU FP6 IST IPHOBAC Integrated Project and ISIS NoE.

\section{References}

[1] A. Hirata et. al., IEEE/OSA JLT, vol. 21(2003), No. 10, pp. 2145-2153.

[2] C. Lim, et. al., IEEE/OSA JLT, vol. 18 (2000), pp. 1355-1363

[3] K. Ikeda, et al. Proc. IMS, vol. 1 (2003), pp. 261-264.

[4] A. Martínez, et al., IEEE Trans. MT\&T., vol. 49 (2001), pp. 2018-2024.

[5] A. Wiberg, et al., IEEE PTL, Vol. 17 (2005), pp. 1938-1940,

[6] J.F. Coward et al., IEEE/OSA J. of Lightwave Technol, vol. 11, pp. 2201, 1993.

[7] W.D. Jemison, et al., IEEE MWCL, vol. 12, pp. 125, 2002.

[8] M.A. Piqueras, et al., IEEE Photonics Technol. Lett., vol. 17, pp. 1947, 2005.

[9] M.A. Piqueras, et al., ECOC’06, vol. 3 (2006), We3.P, pp. 446.

[10] M.A. Piqueras, et al., Proc. OFC 2007, JThA60

[11] R. Sambaraju, et al.,Accepted IEEE/OSA JLT Nov 2007 



\section{Paper G}

Ten gigabits per Second 16-level Quadrature Amplitude Modulated Millimeter-Wave Carrier Generation using Dual-Drive MachZehnder Modulators Incorporated Photonic Vector Modulator

R. Sambaraju, V. Polo, J. L. Corral, and J. Marti

Optics Letters, Vol. 33, no. 16, pages 1833-1835, 2008. 


\title{
Ten gigabits per second 16-level quadrature amplitude modulated millimeter-wave carrier generation using dual-drive Mach-Zehnder modulators incorporated photonic- vector modulator
}

\author{
Rakesh Sambaraju, * Valentín Polo, Juan Luis Corral, and Javier Martí \\ Valencia Nanophotonics Technology Center, Universidad Politécnica de Valencia, Camino de Vera s/n, Ed. IDI-5, \\ 46022 Valencia, Spain \\ *Corresponding author: rsambaraju@ntc.upv.es
}

Received March 17, 2008; revised July 5, 2008; accepted July 7, 2008; posted July 10, 2008 (Doc. ID 93973); published August 7, 2008

A novel photonic-vector modulator architecture for the generation of 16 quadrature amplitude modulation (16 QAM) millimeter-wave carriers using dual-drive Mach-Zehnder modulators is proposed. Experimental generation of 5 Gbits/s 4 amplitude shift-keying (4 ASK) and $10 \mathrm{Gbits} / \mathrm{s} 16$ QAM modulated $42 \mathrm{GHz}$ carriers is reported. The multilevel modulated millimeter-wave signals are demodulated using an electrical receiver and its error-vector magnitude (EVM) estimated from the measurements, obtaining EVMs of -21.04 and $-18.33 \mathrm{~dB}$ for $4 \mathrm{ASK}$ and $16 \mathrm{QAM}$ modulation formats, respectively. (0) 2008 Optical Society of America OCIS codes: $060.5616,060.4088$.

Wireless transmission of multigigabits per second signals has gathered much research effort lately [1-3]. The potential of radio-over-fiber technologies $[4,5]$ to satisfy the stringent bandwidth and flexibility requirements imposed by these challenging communication systems, overcoming the main limitations of purely electrical approaches, has been reported in the literature [6,7]. However, conventional methods based on direct upconversion of the digital baseband signal [1-3] suffer from huge bandwidth constraints, in particular when thinking of realistic full-duplex deployments, where the used bandwidth is required in both downstream and upstream links. Therefore, the use of more spectrally efficient modulation formats such as multilevel quadrature amplitude or multilevel phase-shift keying is of high interest to alleviate such problems [6,7]. Recently, we proposed several photonic-vector modulation (PVM) architectures with low hardware requirements, both optical and electrical, showing the potential of this approach to generate up to $3.6 \mathrm{Gbits} / \mathrm{s} 16 \mathrm{QAM} 40 \mathrm{GHz}$ carriers [8-10].

In this Letter we propose a novel PVM technique employing a single cw laser source, dual-drive MachZehnder modulators (DD-MZMs) combined with a balanced photodetector (BPD) for generating 16 QAM modulated millimeter-wave signals. One of the main advantages of this architecture compared to the previously proposed one for 16 QAM signal generation [9] is that it contains only one optical source, which reduces the total laser relative intensity noise (RIN) contribution to the signal and thus increases the signal-to-noise ratio (SNR). The multilevel signals are generated using the DD-MZMs and not using direct current modulation of the lasers as in [9], which have a lower extinction ratio compared to
Mach-Zehnder (MZ) modulators. The quadrature condition is achieved using an optical delay line as proposed in [8]. The generation of a $10 \mathrm{Gbits} / \mathrm{s} 16$ QAM $42 \mathrm{GHz}$ carrier is reported.

In this PVM scheme, a single cw optical source is modulated with a millimeter-wave carrier using a Mach-Zehnder modulator (MZM) biased at the quadrature bias $(\mathrm{QB})$ point. The millimeter-wave carrier modulated optical signal is split into two arms, $I$ and $Q$, using a $3 \mathrm{~dB}$ power splitter. To induce a $90^{\circ}$ phase shift between the $I$ and $Q$ millimeter-wave carriers, the $Q$ arm optical signal is delayed by $\Delta T$ $=1 / 4 f_{\mathrm{LO}}$ using a tunable optical delay line. This optical delay between the $I$ and the $Q$ arm opticals induces a $90^{\circ}$ phase shift between the photodetected electrical carriers. Two DD-MZMs are used in each arm for modulating baseband data. Both modulators are biased at $\mathrm{QB}$ for generating amplitude modulation; the upper modulator is driven with two independent data $I_{1}(t)$ and $I_{2}(t)$ and the lower with data $Q_{1}(t)$ and $Q_{2}(t)$, where the amplitudes follow the condition $I_{2}=I_{1} / 2 \mathrm{~V}$, and $Q_{2}=Q_{1} / 2 \mathrm{~V}$ for generating four amplitude levels, resulting in 4 ASK signals in each arm. Figure 1 shows a schematic of the PVM archi-

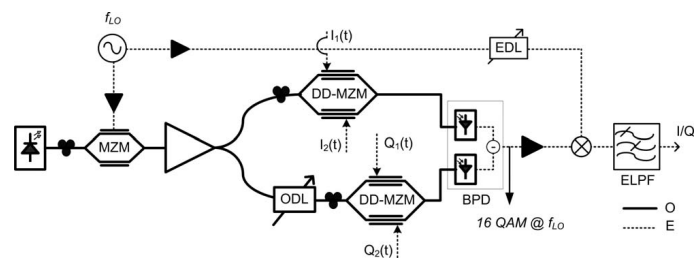

Fig. 1. Schematic of the proposed 16 QAM PVM architecture. 


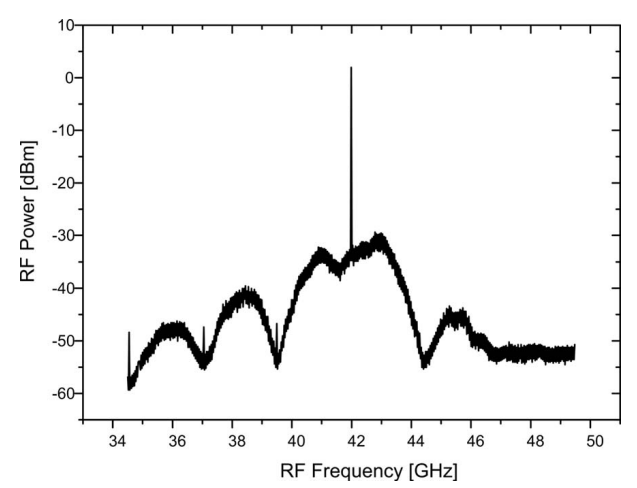

Fig. 2. Radio frequency spectrum at the output of the BPD.

tecture. The upper arm $(I)$ and the lower arm $(Q)$ are photo mixed and combined in a BPD. The electrical output of the photodetector is a 16 QAM modulated millimeter-wave carrier. Equation (1) represents the resulting photocurrent:

$$
\begin{aligned}
i_{\mathrm{PD}}= & \frac{\Re P}{L_{\mathrm{tot}}} J_{0}\left(m_{\mathrm{LO}}\right) J_{1}\left(m_{\mathrm{LO}}\right) \\
& \times\left[\cos ^{2}\left(\frac{\pi}{4 V_{\pi}} I_{1}\left(I_{1}(t)-I_{2}(t)\right)\right) \cos \left(\omega_{\mathrm{LO}} t\right)\right. \\
& \left.+\cos ^{2}\left(\frac{\pi}{4 V_{\pi}} Q_{1}\left(Q_{1}(t)-Q_{2}(t)\right)\right) \sin \left(\omega_{\mathrm{LO}} t\right)\right],
\end{aligned}
$$

where $\mathfrak{R}$ is the responsivity of the photodetector, $P$ is the output power of the laser, $L_{\text {tot }}$ is the total insertion losses, $m_{\mathrm{LO}}=\pi V_{\mathrm{LO}} / V_{\pi}$, and $J_{n}$ is the Bessel function of $n$th order.

A cw DFB laser at $1555.4 \mathrm{~nm}$ with an output power of $+15 \mathrm{dBm}$ is externally modulated by a $42 \mathrm{GHz}$ local oscillator carrier using a $50 \mathrm{GHz}$ MZM biased at the QB point. The output of the MZM is amplified to $+18 \mathrm{dBm}$ using an erbium-doped fiber amplifier (EDFA) to compensate the $6 \mathrm{~dB}$ insertion losses of the modulator and the $3 \mathrm{~dB}$ losses due to $\mathrm{QB}$. The output of the EDFA is divided into two arms using a $3 \mathrm{~dB}$ splitter: upper $(I)$ and lower $(Q)$. The $Q$ arm optical signal is delayed using an optical delay line to generate a $90^{\circ}$ phase shift between the $I$ and $Q$ modulated local oscillator (LO) carrier components. The $I$ and $Q$ optical components were corrected for polarization mismatch using a polarization controller. Two $40 \mathrm{GHz}$ DD-MZMs biased at QB were used for generating the $I$ and $Q 4$ ASK signals. The two arms of the $I$ DD-MZM are driven with two $2.5 \mathrm{Gbits} / \mathrm{s}$ independent data $I_{1}$ and $I_{2}$ where $I_{1}$ was tuned to $1 \mathrm{~V} \mathrm{pp}$ and $I_{2}$ to $0.5 \mathrm{~V}$ pp, resulting in 5 Gbits/s 4 ASK modulation. Similarly the $Q$ arm DD-MZM was driven by $2.5 \mathrm{Gbits} / \mathrm{s} Q_{1}$ and $Q_{2}$ data resulting in another $5 \mathrm{Gbits} / \mathrm{s} 4 \mathrm{ASK}$. The $I$ and $Q$ optical signals with both the baseband data and rf signal modulated on them were photodetected and added in a $45 \mathrm{GHz} \mathrm{BPD}$ with $0.53 \mathrm{~A} / \mathrm{W}$ responsivity. The output of the balanced photodetector (BPD) is a 10 Gbits/s 16 QAM modulated $42 \mathrm{GHz}$ carrier. Figure 2 shows the rf spectrum of the generated 16 QAM $42 \mathrm{GHz}$ carrier.

To analyze the quality generated, the 16 QAM signal was demodulated using an electrical mixer. The 16 QAM signal output at the BPD was amplified and input to a $42 \mathrm{GHz}$ broadband electrical mixer and mixed with the same LO used at the transmitter. The baseband output of the electrical mixer was filtered using an electrical low pass filter with a $3 \mathrm{~dB}$ cut-off frequency of $1.87 \mathrm{GHz}$. The $I$ and $Q$ components of the 16 QAM signal were demodulated electrically by tuning the phase of the LO carrier input to the mixer using a tunable electrical delay. When the electrical delay was tuned to have $0^{\circ}$ phase between the LO and the received $16 \mathrm{QAM}$ carriers, the $I$ signal was demodulated and for $90^{\circ}$ the $Q$ signal was demodulated.

Figures 3(a) and 3(b) show the demodulated $I$ and $Q$ eye diagrams of the $10 \mathrm{Gbits} / \mathrm{s} 16$ QAM millimeter-wave carriers generated by the above described PVM configuration. To analyze the quality of the received signal EVM is calculated from the statistical data of the eye diagrams. To calculate the EVM from the captured 4 ASK $I$ and $Q$ eye diagrams, the histogram of each level was plotted and the mean $\mu$ and the standard deviation $\sigma$ were measured. Later a Gaussian distribution of each symbol was generated using the $\mu$ and $\sigma$ and normalized to 1 . The resulting symbols from the Gaussian distribution were compared with the ideal values for $16 \mathrm{QAM}$ $(1,1 / 3,-1 / 3,-1)$ and the EVM computed. It should be noted that the EVM of the signal is calculated after the receiver stage, and an ideal receiver would improve the EVM. The EVM of the $10 \mathrm{Gbits} / \mathrm{s} 16$ QAM modulated $42 \mathrm{GHz}$ carrier was calculated to be $-18.33 \mathrm{~dB}$. The EVM calculated does not totally reflect the quality of the PVM but contains a contribu-

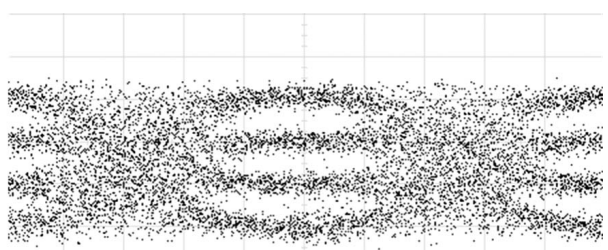

(a)

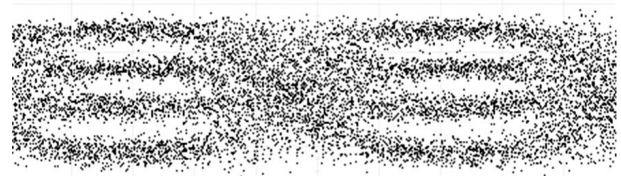

(b)

Fig. 3. Downconverted (a) $I$ and (b) $Q$ components of the 16 QAM signal. 


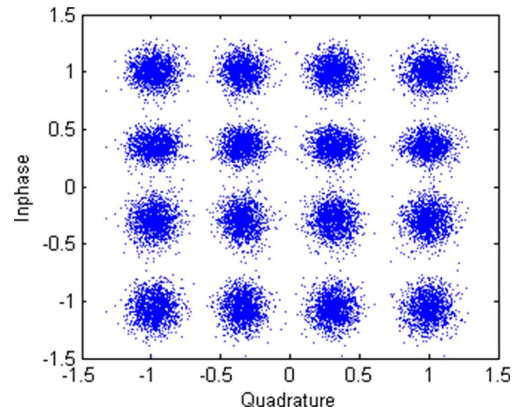

Fig. 4. (Color online) Resulting normalized constellation diagram of the 16 QAM signal.

tion from the electrical demodulation that incorporates bandwidth-limited components. For example, the mixer and the amplifiers have an electrical bandwidth of around $3 \mathrm{GHz}$. Also, the photodetector used in the PVM has an uneven response at $41 \mathrm{GHz}$, which can be noted from Fig. 4. The EVM can be improved by accurate phase matching at the electrical receiver by using a phase-locking mechanism. To prove this claim, instead of vectorial 16 QAM modulation, $5 \mathrm{Gbits} / \mathrm{s} 4 \mathrm{ASK}$ modulation was performed (5 Gbits/s 4 ASK and 10 Gbits/s 16 QAM have the same electrical bandwidth: $5 \mathrm{GHz}$ ) with only one DDMZM and at the same LO frequency, $42 \mathrm{GHz}$. The generated 4 ASK signal's EVM was calculated to be $-21.04 \mathrm{~dB}$, which is a $3 \mathrm{~dB}$ improvement at the same electrical bandwidth.

A novel PVM scheme for generating multigigabits per second 16 QAM modulated millimeter-wave carriers is presented. Generation of a $10 \mathrm{Gbits} / \mathrm{s} 16$ QAM modulated $42 \mathrm{GHz}$ carrier was experimentally demonstrated, and an EVM of -18.33 dB was calculated from measurements. This EVM value can be improved by using an enhanced phase-controlled demodulation that was demonstrated by generating a 5 Gbits/s 4 ASK modulated $42 \mathrm{GHz}$ carrier with an EVM of $-21.04 \mathrm{~dB}$. The advantage of this scheme compared to the previously proposed technique in [9] is that it contains only a single $\mathrm{cw}$ source that reduces the total RIN contribution and increases the SNR.

This work was supported by the European Commission FP6 under project IST-35317 IPHOBAC. Financial support by the Spanish MCyT and EUFEDER under the project TEC2005-08298-C02-02 and the Valencia Regional Government under the project ACOMP2007/144 is gratefully acknowledged.

\section{References}

1. A. Hirata, M. Harada, and T. Nagatsuma, J. Lightwave Technol. 21, 2145 (2003).

2. J. Yu, Z. Jia, L. Yi, Y. Su, G. Chang, and T. Wang, IEEE Photon. Technol. Lett. 18, 265 (2006).

3. A. Wiberg, P. Perez-Milan, M. V. Anders, P. A. Anderkson, and P. O. Hedekvist, IEEE Photon. Technol. Lett. 17, 1938 (2005).

4. A. J. Seeds and J. K. Williams, J. Lightwave Technol. 24, 4628 (2006).

5. J. Capmany and D. Novak, Nat. Photonics 1, 319 (2007).

6. W. D. Jemison, A. J. Kreuzberger, and E. Funk, IEEE Microw. Wirel. Compon. Lett. 12, 125 (2002).

7. P. Candelas, J. M. Fuster, J. Marti, and J. C. Roig, J. Lightwave Technol. 21, 496 (2003).

8. R. Sambaraju, M. A. Piqueras, V. Polo, J. L. Corral, and J. Marti, J. Lightwave Technol. 25, 3350 (2007).

9. R. Sambaraju, M. A. Piqueras, V. Polo, J. L. Corral, and $\mathrm{J}$. Marti, in Proceedings of the European Conference on Optical Communications 2007 (VDE Verlag, 2007), paper Tu5.4.7.

10. M. A. Piqueras, B. Vidal, J. L. Corral, V. Polo, A. Martinez, and J. Marti, IEEE Photon. Technol. Lett. 17, 1947 (2005). 


\section{Paper H}

Transmission of Optically Generated $1.25 \mathrm{~Gb} / \mathrm{s}$ QAM Wireless Signals in a Dynamically Reconfigurable Optical WDM Network

R. Sambaraju, Jose Mora, Beatriz Ortega, and J. L. Corral

Presented at Optical Fiber Communications Conference, OFC 2009, paper OTuJ7, San Diego, March 2009. 


\title{
Transmission of Optically Generated 1.25 Gb/s QAM Wireless Signals in a Dynamically Reconfigurable Optical WDM Network
}

\author{
Rakesh Sambaraju $^{1}$, Jose Mora ${ }^{2}$, Beatriz Ortega ${ }^{2}$, and Juan L. Corral ${ }^{\mathbf{1}}$ \\ Valencia Nanophotonics Technology Center, Universidad Politécnica de Valencia, Camino de Vera s/n, Ed. IDI-5, Acceso A, Nivel \\ 1, 46022 Valencia, Spain. \\ Instituto de telecomunicaciones y Aplicaciones Multimedia, ITEAM, Universidad. Politécnica de Valencia, Camino de Vera s/n, Ed. \\ 8G, 46022 Valencia, Spain. \\ E-mail: rsambaraju@ntc.upv.es \\ Abstract: WDM Transmission of $1.25 \mathrm{~Gb} / \mathrm{s}$ 4-QAM photonic vector modulated signals together \\ with $155 \mathrm{Mb} / \mathrm{s}$ baseband and a UMTS signal in a dynamically reconfigurable network is \\ demonstrated. \\ (C)2008 Optical Society of America \\ OCIS codes: (060.4080) Modulation; (060.5625) Radio frequency photonics; (060.4250) Network
}

\section{Introduction}

With the increase in the capacity of optical networks and the evolution of broadband access networks towards $10 \mathrm{~Gb} / \mathrm{s}$, wireless access of $\mathrm{Gb} / \mathrm{s}$ broadband data is of a great interest [1, 2]. Photonic techniques $[3,4]$ for generation of these broadband wireless links have proven to be a potential candidate considering the various advantages of radio over fibre/optical communication systems like huge bandwidth or easy scalability. Most of these photonic techniques are based on direct up-conversion of baseband data to the RF frequency which is a bandwidth inefficient modulation technique. If wireless access of $10 \mathrm{~Gb} / \mathrm{s}$ data have to be feasible, advanced bandwidth efficient modulation techniques have to be incorporated, and especially when full duplex systems are to be implemented. Recently a new technique called photonic vector modulation (PVM) [5-7] has been proposed for generation of these broadband wireless signals. Main advantages of the proposed PVM as shown in Fig. $1 \mathrm{~b}$ are the direct generation of electrical quadrature modulation from in-phase and quadrature digital streams and LO carrier and the ability to dynamically modify the data rate or RF carrier. Till now, using PVM, $10 \mathrm{~Gb} / \mathrm{s}$ 16-QAM modulated millimetre wave signal generation has been demonstrated [7]. The use of the proposed PVM as compatible technology with future access networks requires the fulfilment of two requirements: the compatibility with state-of-the-art optical networks and its contribution to the cost drift from base stations to central station.

The future access networks should be able to provide broadband connectivity to the user in both fixed and wireless form. To be cost effective, it is required that these radio-over fibre networks can connect various base stations to a single central station while maintaining the base stations as simple as possible. Recently a novel dynamically reconfigurable remote node based on fold-back arrayed waveguide (AWG) incorporating a single optical switch (OS) has been recently proposed for the access networks, with a significant reduction in the number of components [8]. In this paper we experimentally demonstrate the transmission of $1.25 \mathrm{~Gb} / \mathrm{s}$ PVM generated 4-QAM signal with $155 \mathrm{Mb} / \mathrm{s}$ baseband data, and a UMTS signal in a WDM optical network. The different services are routed to their corresponding base stations using the reconfigurable AWG-OS based remote node, and the quality degradation of the services is henceforth measured.

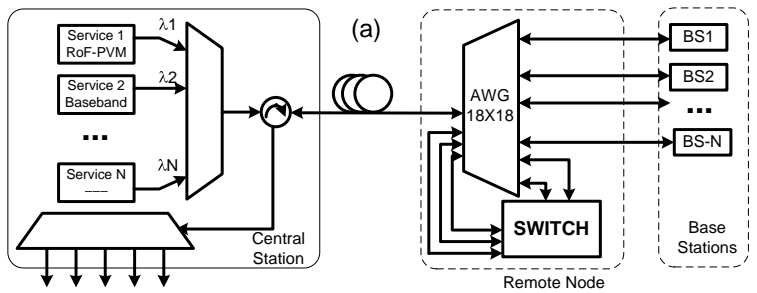

(b)

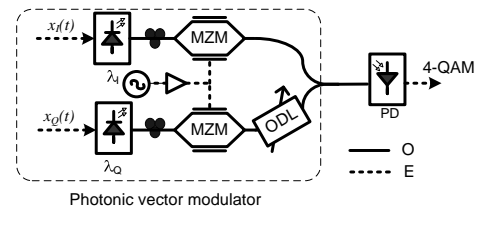

Figure 1: Schematic of the WDM optical network incorporating reconfigurable remote node (a), the schematic of the photonic vector modulator (b).

\section{Reconfigurable Optical Network}

Fig. 1a shows the remote node of the reconfigurable network consisting of a compact and flexible wavelength router based on an optical switch in a foldback AWG as proposed in [8] to symmetrically 
assign upstream/downstream channels to/from central station and base station. In the central station optical channels carrying various services are wavelength division multiplexed and transmitted over a $10 \mathrm{~km}$ SSMF to the remote node. At the remote node the AWG demultiplexes all the wavelengths and routes one own channel per BS, and the extra channels are fed to the input ports of the optical switch. The configuration of the optical switch provides the option of launching the extra channels to the input port of the AWG in order to be routed to corresponding demanding BSs according to the cyclic response. In this system, the services were generated at the central station, demultiplexed at the remote node and routed to the BSs. The three services were $1.25 \mathrm{~Gb} / \mathrm{s}$ 4-QAM at $10 \mathrm{GHz}$ electrical carrier, $155 \mathrm{Mb} / \mathrm{s}$ baseband data with NRZ-OOK modulation, and an UMTS signal. Two base stations were considered in this experiment BS1 and BS2. The PVM generated signals were assigned as own channel to the BS1, and the UMTS signal as own channel to BS2. The $155 \mathrm{Mb}$ /s baseband digital signal was used as an extra channel which could be rerouted to any one of the two operating base stations using the reconfigurable remote node configuration. Fig 2 shows the optical spectra at the base stations under different scenarios.

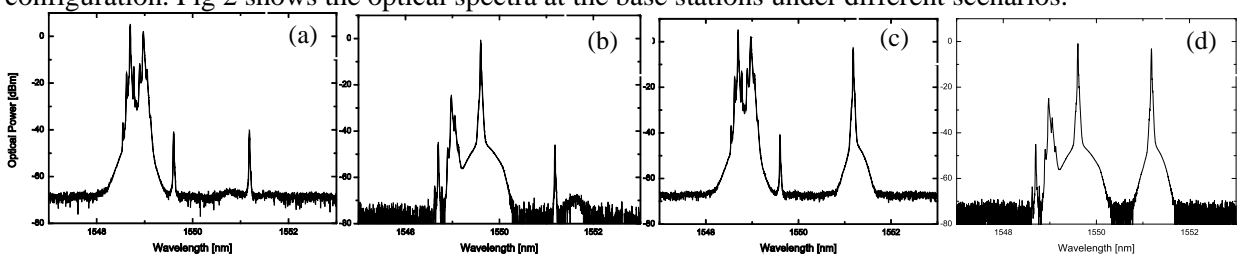

Figure 2: Optical spectra at the BS1, when the $155 \mathrm{Mb} / \mathrm{s}$ extra channel is at BS2 (a), and BS1 (c). Optical spectra at the BS2, when

\section{Experimental Setup}

the $155 \mathrm{Mb} / \mathrm{s}$ extra channel is at BS1 (b), and BS2 (d).

An optical channel centred at $1548 \mathrm{~nm}$ was used to transmit the $1.25 \mathrm{~Gb} / \mathrm{s}$ PVM generated $10 \mathrm{GHz}$ 4-QAM signals. To generate the 4-QAM signals the schematic shown in Fig 1b [6] is used. Two DFB lasers $\left(\lambda_{I}=1548.73 \mathrm{~nm}, \lambda_{Q}=1549.03\right)$ with a modulation bandwidth of $1 \mathrm{GHz}$ were directly current modulated by two $625 \mathrm{Mb} / \mathrm{s}$ NRZ sequence. The two optical carriers were independently modulated with a $10 \mathrm{GHz}$ LO signal by two Mach-Zehnder modulators biased at their quadrature bias points. The $Q$-arm optical carrier was delayed by 25 ps using an optical delay line, where the delay corresponds to 90 degrees phase shift between the $I$ and $Q$ carriers. For the UMTS signal, an arbitrary waveform generator was used to generate the UMTS signal, and modulated on a $1549.6 \mathrm{~nm}$ optical carrier using a conventional Mach-Zehnder modulator. Similarly a $155 \mathrm{Mb} / \mathrm{s}$ PRBS signal was NRZ-OOK modulated on a $1551.2 \mathrm{~nm}$ optical carrier using another MZ modulator. The three signals were multiplexed using a 4:1 optical combiner and transmitted over a $10 \mathrm{~km}$ SSMF.

To measure the quality of the PVM generated signal at the BS1, an electrical mixer was used. A copy of the LO was launched into the LO port of the broadband mixer, and the photo detected RF signal at the RF port. Using an electrical phase shifter in the LO, the phase was tuned and the $I$ and $Q$ baseband data were demodulated. Similarly the UMTS signal quality was measured using a vector signal analyzer. The $155 \mathrm{Mb} / \mathrm{s}$ baseband data was photodetected and the Q-factors were measured and the BER calculated by using a digital signal analyzer.

\section{Results}

Initially the PVM generated 4-QAM signals were demodulated in a back-to-back (B2B) case and the Q-factors of the demodulated $I$ and $Q$ data were measured by setting the optical detected power at +5 $\mathrm{dBm}$ with an EDFA and an optical attenuator. Q-factors of $17.8 \mathrm{~dB}$ and $16 \mathrm{~dB}$ were obtained for $I$ and $Q$ data respectively, which difference is mainly due to the non-identical lasers employed for each signal component. Similarly after $10 \mathrm{~km}$ of fibre transmission, the Q-factors were measured as $16.5 \mathrm{~dB}$ and $13.5 \mathrm{~dB}$ for $I$ and $Q$ respectively. In both cases, the B2B and transmitted signal through $10 \mathrm{~km} \mathrm{SSMF}$, measurements show remarkable eye opening offering an excellent quality for digital wireless communications. The degraded Q-arm Q-factor was mainly due to the broad line width of the Q-arm laser at 1549.03 after direct modulation, combined with the fibre chromatic dispersion. For the sake of comparison, instead of 4-QAM signals, ASK signals were generated by simply switching off one of the lasers in the PVM. In this case no vector modulation is performed; rather direct up-conversion of baseband data to the RF frequency which occupies the same electrical bandwidth. When the laser $\lambda_{I}$ of the PVM was used to generate the ASK signal, it is denoted as ASK-I, else ASK-Q. Q-factors in a B2B measurement of 18.4 and $17.6 \mathrm{~dB}$ for ASK-I and ASK-Q, respectively.

In a multiservice scenario, other than the PVM generated signals, UMTS and baseband digital signals were multiplexed and transmitted to the remote node. Before demultiplexing and routing the services to corresponding base stations, the WDM optical signal was amplified using an EDFA. The PVM signals 
were routed to BS1, the UMTS to the BS2, and the $155 \mathrm{Mb} / \mathrm{s}$ as an extra channel which was routed one at a time to both base stations. Table I contains the Q-factors of the PVM generated signals at the BS1 when the $155 \mathrm{Mb} / \mathrm{s}$ baseband signal was routed either to BS1 or BS2, showing similar and independent values of the reconfigurable channel assignment scenario.

Table I: Photodetector input power and Q-factors of the demodulated signals under different scenarios with multiple services

\begin{tabular}{|c|c|c|c|c|}
\hline Modulation $>$ & 4-QAM (I) & 4-QAM (Q) & ASK-I & ASK-Q \\
\hline Pin $[\mathrm{dBm}]>$ & 1.5 & 1.5 & -1.7 & -1.7 \\
\hline Q-factor $[\mathrm{dB}]:$ extra at BS1 & 13.6 & 9.9 & 17.8 & 15.9 \\
\hline Q-factor [dB]: extra at BS2 & 13.4 & 9.6 & 18.1 & 16.3 \\
\hline
\end{tabular}

The low Q-factors at the BS were also due to the fact that BS1 has another AWG to separate different services reaching the BS1, which results in additional optical losses. The quality of the UMTS signal was measured at BS2 similarly in two scenarios: when the $155 \mathrm{Mb} / \mathrm{s}$ was at BS1 or BS2. Fig. 3b shows the EVM of the UMTS signal plotted against the received optical power. The results show EVM factors below $1.2 \%$ satisfying the 3GPP standards at a received optical power of $-14 \mathrm{dBm}$.. Similarly the quality of the $155 \mathrm{Mb} / \mathrm{s}$ baseband digital signal was assessed at the BS1 by calculating the BER as shown in the Fig. 3a. Similar results were found when measured at BS2, showing that the QoS is independent of the routing scenario.
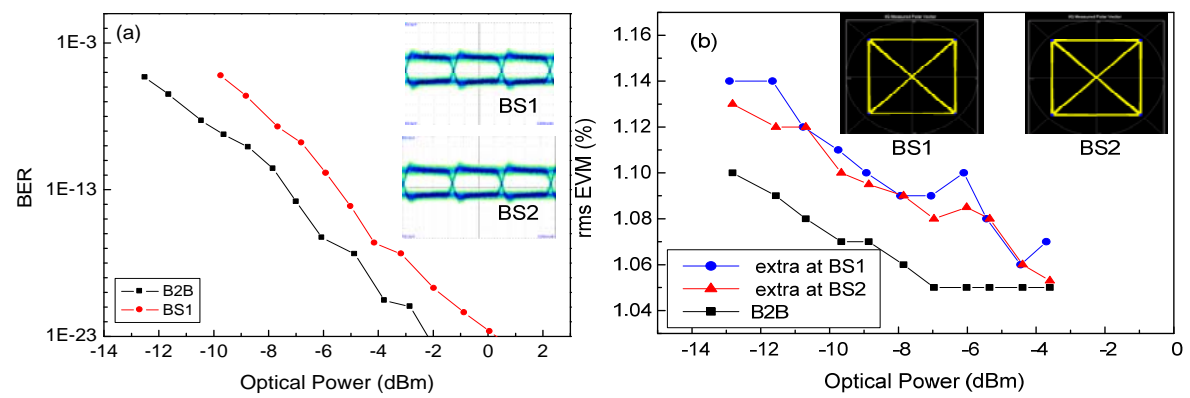

Figure 3: Calculated bit error ratio of the extra $155 \mathrm{Mb} / \mathrm{s}$ digital signal at BS1, corresponding B2B case, and the eye diagrams at the two base stations (a), and EVM of the UMTS signal at BS2 in B2B case, when extra is at BS1 and BS2, and the resulting constellation diagrams (b).

\section{Conclusions}

In this paper the feasibility of a PVM generated signal being transmitted over $10 \mathrm{~km}$ fibre, dynamically routing using a reconfigurable remote node, and co-existing with other analogue and digital services was experimentally demonstrated. The advantage of this optical network incorporating the reconfigurable remote node is that all the generating equipment is at the central station relaxing the BS complexity. It was also demonstrated that the quality of the various services are not affected by the other services, and does not either depend on the different routing scenarios.

\section{Acknowledgment}

This work has been supported by the European Commission FP6 under project IST-35317 IPHOBAC, and FP7 under project ALPHA (grant no. 212352). Financial support by the Spanish MCyT and EUFEDER under the project TEC2005-08298-C02-02, and TEC2005-08298-C02-01 (ADIRA) and the Valencia regional government under the projects ACOMP2007/144 and ACOMP2007/207 are greatly acknowledged.

\section{References}

[1]V. Dayadyuk et al, IEEE MTT-S, vol. 55, no. 12, pp. 2813-2820, 2007.

[2] M. Hirata et al, IEEE/OSA JLT., vol. 21, no. 10, pp. 2145-2153, 2003.

[3] A. J. Seeds and J. K. Williams, IEEE/OSA JLT, vol. 24, no. 12, pp. 4628-4641, 2006.

[4] J. Capmany and D. Novak, Nature Photonics, vol. 1, pp. 319-330, 2007.

[5] M.A. Piqueras, et al, IEEE PTL, vol. 17, no. 9, pp. 1947-1949, September 2005.

[6] R. Sambaraju et al, IEEE/OSA JLT, vol. 25, no. 11, pp. 3350-3357, 2007.

[7] R. Sambaraju et al, Opt. Lett., vol. 33, no. 16, pp. 1833-1835, 2008.

[8] B. Ortega et al, Opt. Exp., vol. 15, no. 25, pp. 16781-16786, 2007. 


\title{
Paper I
}

\section{Photonic Vector Demodulation of 2.5 Gbit/s QAM Modulated Wireless signals}

\author{
R. Sambaraju, J. Palaci, R. Alemany, V. Polo, and J. L. Corral
}

In International Topical Meeting on Microwave Photonics, 2008. Jointly held with the 2008 Asia-Pacific Microwave Photonics Conference. MWP/APMP 2008., pages 117-120, Gold Coast Australia, Sept. 2008. 


\title{
Photonic Vector Demodulation of 2.5 Gbit/s QAM Modulated Wireless Signals
}

\author{
Rakesh Sambaraju, Jesus Palaci, R. Alemany, Valentin Polo, and Juan Luis Corral \\ Valencia Nanophotonics Technology Center, Universidad Politecnica de Valencia \\ Camino de Vera S/N, Ed. IDI-5, Accesso-A, Valencia, 46022, Spain. \\ rsambaraju@ntc.upv.es
}

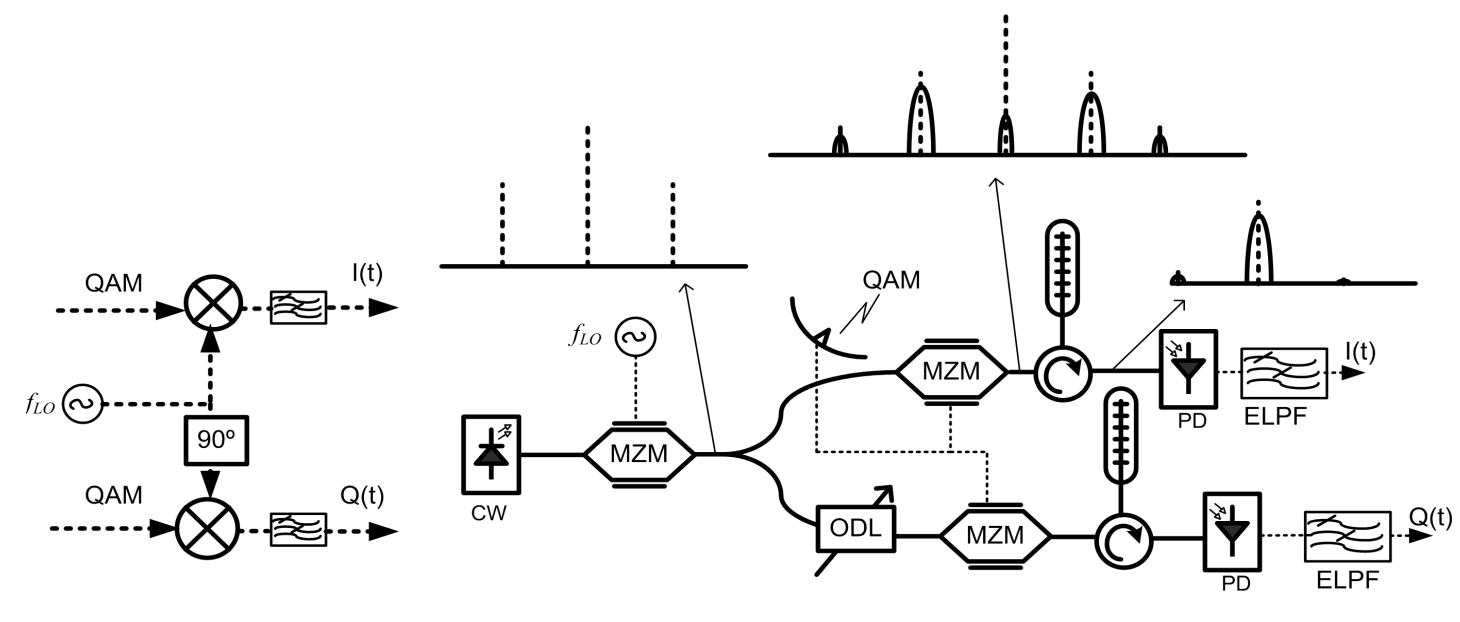

(a)

(b)

Figure 1. Schematic of a typical electrical vector demodulator (a), and the schematic of the proposed photononic vector demodulator (b).

\begin{abstract}
In this paper, a technique for demodulation of vector modulated signals like QAM, using photonic techniques is presented. The photonic vector demodulator is capable of converting broadband wireless QAM signals into independent Inphase and Quadrature optical baseband signals. Experimental demonstration of demodulation of $2.5 \mathrm{Gbit} / \mathrm{s}$ QAM modulated $20 \mathrm{GHz}$ carrier is presented. Bit error ratio of the demodulated-I and -Q optical baseband signals is measured and an error free demodulation achieved. A receiver sensitivity of $-26.5 \mathrm{dBm}$ and $27 \mathrm{dBm}$ were measured for the $I$ and $Q$ signals respectively.
\end{abstract}

\section{INTRODUCTION}

Broadband access networks are evolving towards $10 \mathrm{G}$ connectivity provision. The possibility of accessing such high data rates using wireless links is of a great interest due to the various advantages of wireless links [1]. Also these broadband wireless links are an appropriate solution for providing network redundancy under disaster or service disruption situations [2]. Another important application of these broadband wireless links is to implement the wireless extension to the existing FTTH networks, known as FTTA fibre to the air. Radio-over-fibre technologies offer the advantage of huge bandwidth and flexibility to implement these millimeter wave broadband links $[3,4,5]$.

Recently, some research groups have demonstrated high bandwidth millimeter wave wireless links using photonic techniques, and up to $10 \mathrm{Gbit} / \mathrm{s} 120 \mathrm{GHz}$ carrier generation was demonstrated [6,7]. However, these techniques were based on direct up-conversion of digital baseband signal which suffer from bandwidth inefficiency. Therefore, efforts to generate bandwidth efficient modulation formats like multilevel quadrature amplitude or phase modulation 
(MQPSK/MQAM) are of high interest [8]. Recently, using photonic vector modulation techniques, generation of Gbit/s QAM modulated mm-wave carriers, and up to $10 \mathrm{Gbit} / \mathrm{s}$ 16QAM modulated $40 \mathrm{GHz}$ carrier were reported [9, 10]. For demodulation of these high capacity vector modulated wireless carriers, a photonic vector demodulator is presented. Apart from the various advantages of photonic technology compared to conventional electrical technology, PVdM offer an advantage of converting the wireless signals into baseband optical signals to be directly transmitted in a fibre network.

In this paper a novel photonic vector demodulation (PVdM) technique for demodulation of QAM modulated wireless signals is presented. Experimental demonstration of demodulation of $2.5 \mathrm{Gbit} / \mathrm{s}$ QAM modulated $20 \mathrm{GHz}$ carrier is reported. The output of the PvDM is two baseband ASK modulated optical signals.

The organization of this paper is as follows: section I describes the working principle, the section II the experimental setup, and its various blocks. Section III describes the results, and conclusion in section IV.

\section{WORKING PRINCIPLE}

Demodulation of vector signals like QAM require the demodulation of the $I$ and $Q$ carrier components. In an electrical vector signal demodulator, the received wireless signal with QAM modulation is mixed with a LO carrier to obtain the $I$ baseband data, and the RF signal is mixed with a $90^{\circ}$ phase shifted LO carrier for obtaining the $Q$ baseband component. Similarly, in a photonic vector demodulator the same principle is applied, but using an optical carrier. The LO is first modulated on a continuous wave optical carrier using an external modulator. The LO modulated optical carrier is divided into two arms named $I$ and $Q$. An optical delay line is used in the arm Q to delay the optical signal by $1 / 4 f_{L O}$. This optical delay also delays the LO carrier modulated, which is also a $90^{\circ}$ phase shift compared to the $I$ arm. The delay does not depend of the transmitter design, since LO at the receiver will be in phase with the received RF signal, and the optical delay is only used to induce an additional $90^{\circ}$ phase shift. The working principle of the photonic vector modulator is shown in Fig. 1.

The optical carrier and the sidebands separated by $f_{L O}$ output at the first MZM (Fig. 1) are modulated by the RF QAM signal using another external modulator. The output of the second external modulators is an optical carrier with the mixed RF and LO carriers modulated on it. Later, a fibre Bragg grating is used to chose one of the optical sidebands with only the baseband data modulated on it. The output of the fibre grating is an optical amplitude modulated baseband signal. Depending on the phase of the LO modulated on the optical carrier by the first external modulator, the output of the filter is either $I$ (for $0^{\circ}$ phase), or $Q$ for $\left(90^{\circ}\right.$ phase) baseband data.

\section{EXPERIMENTAL SETUP}

The experimental setup of the photonic vector demodulator consists of three major blocks as shown in Fig. 2. It consists of the electrical QAM signal generator, photonic vector demodulator, and an optical pre-amplified receiver for measuring the bit error ratio.

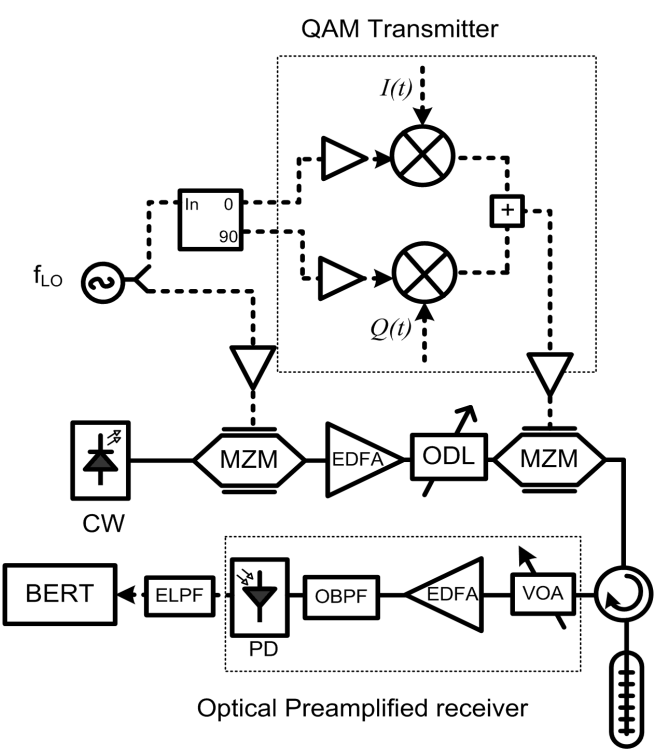

Figure 2. Experimental setup of the photonic vector modulator containing an electrical QAM transmitter and an optical pre-amplified receiver.

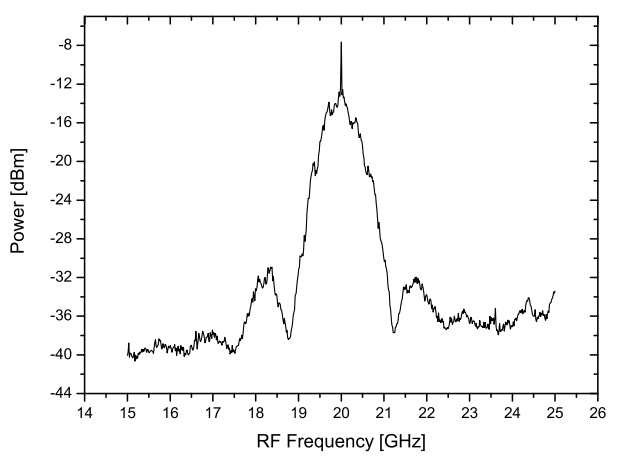

Figure 3. RF spectrum output at the QAM transmitter.

\section{A. Electrical QAM Signal Generator}

The electrical QAM signal was a $2.5 \mathrm{Gbit} / \mathrm{s}$ QAM modulated $20 \mathrm{GHz}$ carrier. The choice of the data rate, and the carrier frequency was determined by the available RF components. 
For generating the QAM signal two electrical mixers were used to mix the $I$ and $Q$ data with two LO carrier components. Two $1.25 \mathrm{Gbit} / \mathrm{s} 2^{31}-1$ PRBS data sequences were mixed with the LO carriers amplified to $+22 \mathrm{dBm}$, where the $Q$ arm LO carrier was $90^{\circ}$ phase shifted using an electrical 3-dB hybrid. The two RF signals were combined resulting in a $2.5 \mathrm{Gbit} / \mathrm{s}$ QAM modulated $20 \mathrm{GHz}$ carrier. The generated QAM signal was amplified to $+12.5 \mathrm{dBm}$ using a low noise amplifier. Fig. 3 shows the RF spectrum of the QAM signal.

\section{B. Photonic Vector Demodulator}

A continuous wave laser with an output power of $+6 \mathrm{dBm}$ at $1550.15 \mathrm{~nm}$ of wavelength was used as the optical carrier. A copy of the $20 \mathrm{GHz} \mathrm{LO}$ used for generating the electrical QAM signal is used to modulate the optical carrier using a 50 $\mathrm{GHz}$ Mach-Zehnder modulator biased at its quadrature bias point. Later an Erbium-doped optical amplifier was used to amplify the optical signal to $+3 \mathrm{dBm}$. An optical delay line was followed by the EDFA to tune the delay for changing the RF phase. The ODL was used to make sure that the phase difference between the LO and the RF signal was maintained either $0^{\circ}$ or $90^{\circ}$, and also to compensate any extra delay added by the optical path. Another $50 \mathrm{GHz}$ bandwidth MZM biased at its quadrature bias point was used to modulate the QAM RF signal. The QAM signal is modulated on the two optical

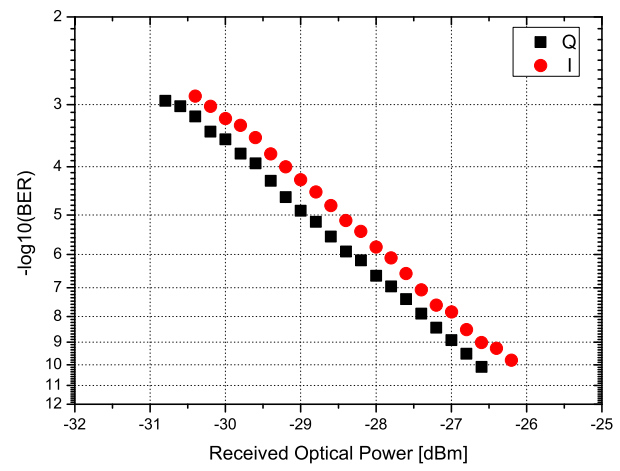

Figure 4. The measured bit error ratio (BER) of the demodulated I and Q optical baseband data plotted against average optical received power.

sidebands of the carrier. Depending on the phase difference between the LO carrier (tuned by the ODL) and the RF signal, the data modulated on the optical sidebands is either $I$ $\left(0^{\circ}\right)$ or $Q\left(90^{\circ}\right)$.

A fiber Bragg grating with center frequency of $1550 \mathrm{~nm}$ with a bandwidth of $25 \mathrm{GHz}$ was used at the output of the second MZM to filter out the lower sideband. The output of the filter is an optical $1.25 \mathrm{Gbit} / \mathrm{s}$, amplitude modulated baseband signal. The demodulated electrical $I$ and $Q$ data eye diagrams are shown in Fig. 5

\section{Optical Pre-amplifier Receiver}

To measure the bit error ratio of the optical signal, an optical pre-amplified receiver was used. The output of the FBG was at $-18 \mathrm{dBm}$, and amplified using an EDFA and followed by a tunable optical bandpass filter centered at 1550 $\mathrm{nm}$ with $1 \mathrm{~nm}$ of bandwidth. A variable optical attenuator was placed between the FBG and the EDFA to measure the receiver sensitivity of the signal. The optical baseband signal

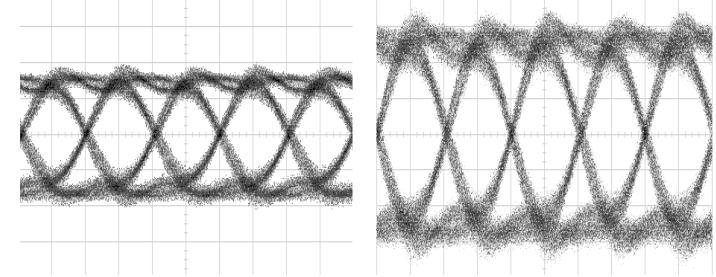

Figure 5. The electrical eye diagarams at the output of the photodiode with an input power of $-18 \mathrm{dBm}$ to the optical pre-amplifier. Left (I) and right (Q).

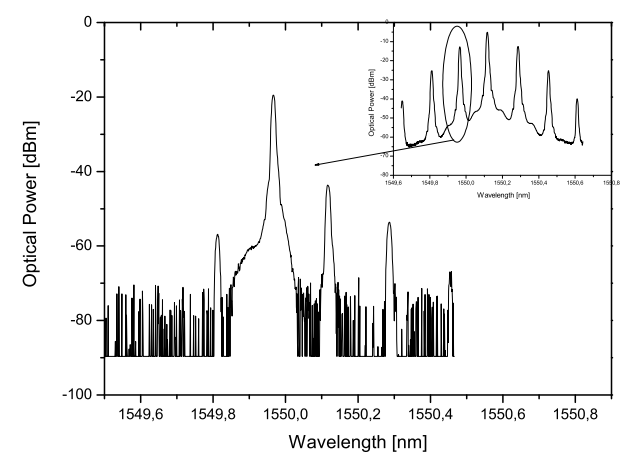

Figure 6. The optical spectrum at the output of the FBG. Inset is the optical spectrum at the input of the FBG.

was photo-detected using a $12.5 \mathrm{Gbit} / \mathrm{s}$ photo-receiver with integrated amplifier in it. The demodulated baseband electrical data was low-pass filtered using a $900 \mathrm{MHz}$, low pass filter before prior to bit error ratio measurement.

\section{RESULTS AND DISCUSSION}

Fig. 4 shows the bit error ratio plots of the $I$ and $Q$ data plotted against the average optical power received at the preamplifier receiver. Error free transmission was obtained with received power as low as $-25 \mathrm{dBm}$. To obtain a bit error ratio of $10^{-9}$, received average optical power of $-26.5 \mathrm{dBm}$ and -27 $\mathrm{dBm}$ for $I$ and $Q$ respectively. It can be concluded from the BER curves that typical off the shelf devices for optical baseband communications can be used for demodulation of QAM RF signals. The performance of the photonic vector 
demodulator was mainly limited by the availability of the RF components, and the bandwidth of the FBG. Ideally the RF frequency should be much greater than the bandwidth of the FBG to avoid interference from the other optical carriers. The $\mathrm{RF}$ frequency in this experimental demonstration was chosen as $20 \mathrm{GHz}$, and the FBG had a bandwidth of $25 \mathrm{GHz}$, resulting in interference from other bands as shown in Fig. 6.

\section{CONCLUSION}

A novel technique for demodulation of QAM mm-wave carriers using photonic techniques is presented. The photonic vector demodulator is able to convert a QAM modulated wireless signals into two ( $I$ and $Q$ ) ASK modulated optical baseband signals. An error free demodulation of $2.5 \mathrm{Gbit} / \mathrm{s}$ QAM modulated $20 \mathrm{GHz}$ carrier was demonstrated using the photonic vector demodulator. Optical receiver sensitivity at a BER of $10^{-9}$ was measured as $-27 \mathrm{dBm}$ and $-26.5 \mathrm{dBm}$ for $I$ and $Q$ data respectively. The ability of the photonic vector demodulator to demodulate and transform a QAM wireless signal to independent optical ASK modulated baseband signal makes it a potential choice for wireless access to a FTTH network.

\section{REFERENCES}

[1] P. Stuckmann, and R. Zimmermann, "Towards ubiquitous and unlimited capacity communication networks: european research in framework programme 7," IEEE Comm. Mag., vol. 45, no. 5, pp. 148147, 2007.

[2] C. P. Liu, et al "Full-duplex DOCSIS/wirelessDOCSIS fiber-radio network employing packaged AFPMs as optical/electrical transducers," J. Lightwave Technol., vol. 25, no. 3, pp.673-684, 2007.

[3] A. J. Seeds, and J. K. Williams, “ Microwave photonics," J. Lightwave Technol., vol.24, no. 12, pp. 4628-4641, 2006.

[4] J. Capmany, and D. Novak, "Microwave photonics combines two worlds," Nature Photonics, vol. 1, no. 3, pp. 319-330, Jun 2007.

[5] A. Stohr, et al, " $60 \mathrm{GHz}$ radio-over-fiber techniques for $10 \mathrm{~Gb} / \mathrm{s}$ broadband wireless transmission," Wireless World Research Forum, 22-24 April 2008, Ottawa, Canada, 2008, (accepted)

[6] A. Hirata, M. Harada, and T. Nagatsuma, "120-GHz wireless link using photonic techniques for generation, modulation, and emission of millimeter-wave signals," J. Lightwave Technol., vol. 21, no. 10, pp. 2145-2153, 2003.

[7] R. W. Ridgway, and D. W. Nippa, "Generation and modulation of a 94$\mathrm{GHz}$ signal using electrooptic modulators," IEEE Photon. Tech. Lett., vol. 20 , no. 8 , pp. 653-655, 2008.

[8] R. sambaraju, et al, "generation of multi-gigabit-per-second MQAM/MPSK-modulated millimeter-wave carriers employing photonic vector modulator techniques," J. Lightwave Technol., vol. 25, no. 11, pp-3350-3357, 2007.

[9] R. Sambaraju, et al, "Photonic vector modulation of $3.6 \mathrm{~Gb} / \mathrm{s} 16 \mathrm{QAM}$ at $39 \mathrm{GHz}$ for radio-over-fibre systems," in Proc. European Conference on Optical Communications ECOC 2007, paper Tu. 5.4.7, Berlin 2007.

[10] R. Sambaraju, et al, "16-QAM $10 \mathrm{~Gb} / \mathrm{s} \mathrm{mm-wave} \mathrm{wireless} \mathrm{links}$ employing photonic vector modulation techniques," in ICT MobileSummit 2008 (accepted). 



\section{Paper J}

Differential Phase Modulated Wireless/wireline Links Based on Optical Phase Modulation and Demodulation

R. Sambaraju, R. Alemany, A. Hakansson, and J. Herrera

to be presented in IEEE Photonics Society Annual Meeting., paper ThM4, Denver CO, Nov. 2010. 


\section{Differential Phase Modulated Hybrid Wireless/Wireline Links based on Optical Phase Modulation and Demodulation}

\author{
Rakesh Sambaraju, Ruben Alemany and Javier \\ Herrera \\ Universidad Politecnica de Valencia, Camino de Vera S/N, \\ Valencia - 46022, Spain. \\ Email: r.sambaraju@ieee.org
}

\author{
Andreas Hakansson \\ DAS Photonics S.L, Camino de Vera S/N, Valencia - \\ 46022, Spain. \\ Email: ahakansson@dasphotonics.com
}

\begin{abstract}
Wireless links based on optical DxPSK and passive optical interferometer based demodulation is presented and a 5 $\mathrm{Gb} / \mathrm{s} 60 \mathrm{GHz}$ DPSK wireless signal generation and demodulation experimentally demonstrated.
\end{abstract}

Keywords; Coherent communication, digital receievrs, digital signal processing, microwave photonics

\section{INTRODUCTION}

Optical access networks with their FTTH standards are the current state of the art access technology, but in many scenarios like rural areas or new green field areas the last mile fiber installation might be expensive or just logistically challenging. Wireless links in the millimeter wave frequency ranges offer good solution for extending the reach of the existing fiber networks. These high capacity wireless links have other applications like inter-building communication links, military, emergency services etc. The millimeter wave frequency bands like $60 \mathrm{GHz}, 70 / 80 \mathrm{GHz}$ are of special interest since these bands have a few $\mathrm{GHz}$ of bandwidth available. These wireless links mostly connect two remote antenna units (RAUs), where the $\mathrm{Gb} / \mathrm{s}$ signals must be transported to/from a central station (CS). One solution is to optically transport the $\mathrm{Gb} / \mathrm{s}$ baseband signal to the RAU and electrically up-convert the signal to the desired frequency and similarly electrically demodulate the wireless signals at the receiver side and transport the baseband signals optically to the CS. The disadvantage of this solution is complicated RAU architecture which increases the operation costs.

Since fibre optics are needed to transport/remote the $\mathrm{Gb} / \mathrm{s}$ signals, a better solution would be to integrate the upconversion/demodulation process into optics at the CS, and keeping the RAUs as simple as possible. Radio over fibre
(RoF) [1] technologies offers the bandwidth and flexibility for providing millimetre-wave $\mathrm{Gb} / \mathrm{s}$ wireless links. To integrate the current RoF links with the existing optical access networks, the RoF links should be dispersion tolerant [2, 3], and passive in nature. In this paper, we present a single side band (SSB) RoF link with optical differential phase shift keying (DxPSK). The DxPSK wireless signals are received at the RAU, where another RoF link transmits the signals to the $\mathrm{CS}$. Using the phase preserving properties of the RF signal modulation on an optical carrier [4], the original DxPSK modulation is restored at the receiver, and a 1-bit Delay MachZehnder Interferometer (DMZI) was used to perform the optical demodulation, which otherwise [5] would need sophisticated electrical phase locking techniques. This proof of concept opens new opportunities for RoF wireless systems, where modulation formats like DQPSK [6] can be implemented without any millimetre-wave phase locking, just by using passive DMZI demodulation. Moreover, Silicon Photonics offers here an excellent platform for monolithic system integration on a chip. Apart from the laser, all components can be integrated directly on a single die using standard CMOS technology, offering a mass production low cost solution [7]

\section{WORKING PRINCIPLE}

Figure 1 shows the schematic of the experimental setup. The setup is divided into 4 parts. First at the transmitter CS, a 1550 $\mathrm{nm}$ optical carrier emitted from an external cavity laser (ECL) is modulated by a $30 \mathrm{GHz}$ local oscillator signal using a MZM biased at its minimum transmission point. The output of the MZM is an optical carrier suppressed DSB, with two coherent optical carriers $60 \mathrm{GHz}$ apart. The two optical carriers are amplified using an Erbium Doped Fibre Amplifier (EDFA).

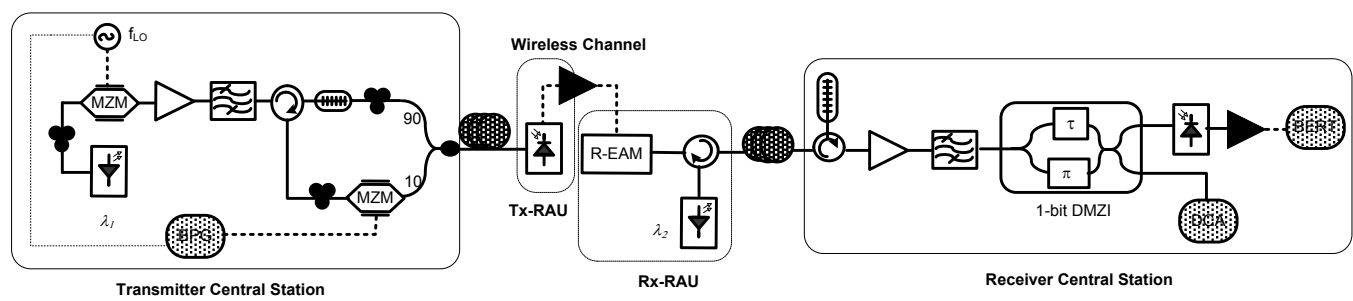

Fig. 1: Experimental set-up for QPSK wireless-over-fibre link 


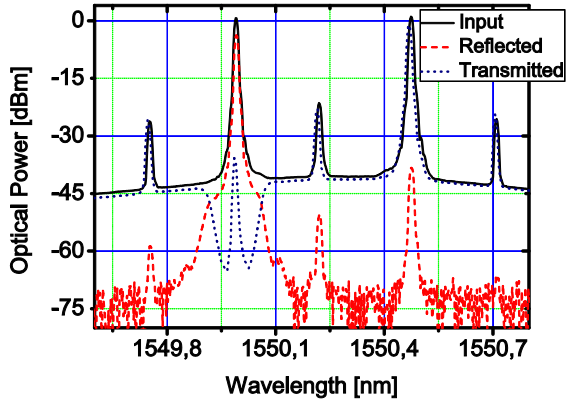

Fig. 2: Optical spectra of the input, reflected and transmitted signal at the FBG at transmitter central station

To filter out the excess out of band noise, a 1-nm tunable optical band-pass filter was used. The two optical carriers were de-multiplexed using a $1550 \mathrm{~nm}$ centred Fibre Bragg Grating (FBG).

Figure 2 shows the optical spectra at the input, reflected and transmitted ports of the FBG. The reflected signal after passing through a circulator is input into a $10 \mathrm{GHz} \mathrm{MZ}$ modulator. A $5 \mathrm{~Gb} / \mathrm{s} \mathrm{NRZ} \mathrm{data} \mathrm{with} 7 \mathrm{Vpp}$ was modulated on the optical carrier with the MZ modulator biased at minimum transmission resulting in a DPSK signal. The other unmodulated optical carrier is added with $90 \%$ of the DPSK modulated signal using a 10:90 optical coupler. An asymmetric coupling ratio was chosen to compensate the additional loss from data MZM. The combined optical signals, the DPSK modulated optical carrier, and the $60 \mathrm{GHz}$ separated un-modulated carrier are transmitted over a 1-km fibre to a 75 $\mathrm{GHz}$ bandwidth photo detector (PD) located at the Tx-RAU.

At the Rx-RAU, the $5 \mathrm{~Gb} / \mathrm{s} 60 \mathrm{GHz}$ DPSK signals output from the $\mathrm{PD}$ are amplified using a $26 \mathrm{~dB}$ gain high power amplifier, and modulated on another optical carrier emitting at $1555 \mathrm{~nm}$ from an ECL, using a $60 \mathrm{GHz}$ Reflective ElectroAbsorption Modulator (R-EAM). An optical circulator was used between the ECL and R-EAM because of the reflective nature of the EAM. The output at the port 3 of the circulator is a DSB signal with the central carrier, and the DPSK signal modulated on the sidebands. The DSB optical signal is transmitted to the receiver CS using another optical fibre of a $1 \mathrm{~km}$. At the receiver CS, another FBG centred at $1555 \mathrm{~nm}$ with a bandwidth of $25 \mathrm{GHz}$ is used to filter one of the sidebands. The filtered sideband passes through a variable optical attenuator (VOA) and an EDFA, which acts as a typical pre-amplified optical receiver. The amplified signals are filtered to reduce the out of band noise using a 1-nm tunable optical bandpass filter. To demodulate the DPSK signals, a $10 \mathrm{GHz}$ 1-bit DMZI in combination with singleended photo-detection was used. The photo-receiver was a 10 $\mathrm{GHz} \mathrm{PD}$ with a trans-impedance amplifier.

\section{RESULTS}

To evaluate the penalty induced by the whole process of

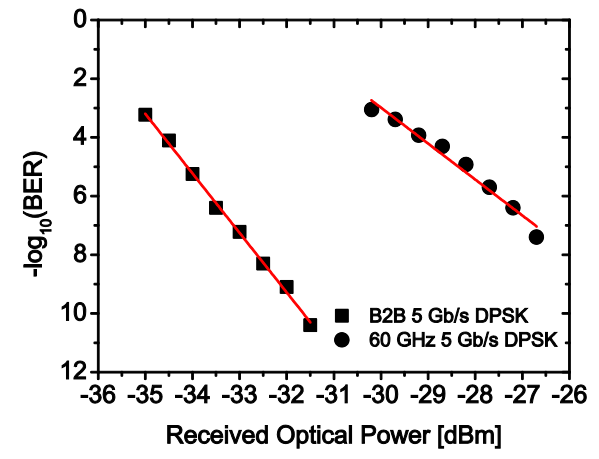

Fig. 4: BER plots of both back-to-back optical DPSK signals and $60 \mathrm{GHz}$ up-converted signals.

transmission and up-conversion of the $5 \mathrm{~Gb} / \mathrm{s}$ DPSK signal to $60 \mathrm{GHz}$, and the other way around, first the Bit Error Ratio (BER) of the $5 \mathrm{~Gb} / \mathrm{s}$ signal was measured in a back-to-back (B2B) scenario using a typical optical pre-amplified receiver. For the B2B case, the BER was measured at the output of the DPSK MZM as shown in the fig. 1. For the $60 \mathrm{GHz}$ case, the BER was measured as a function of the received optical power by varying the attenuation of the VOA at the receiver CS. Both the results are plotted in fig. 3. Results show a very good performance of the proposed differential phase modulated millimetre wave wireless system, considering that the results include fibre transmission and frequency up- and downconversion. Receiver sensitivity at the receiver CS of around $27.5 \mathrm{dBm}$ was measured for a BER of $10^{-6}$. Comparing with the $\mathrm{B} 2 \mathrm{~B}$ case, a penalty of $6 \mathrm{~dB}$ is observed. In the described measurements, just one RF amplifier is used, and a rough calculation of the power budget for a typical wireless link with high gain antennas and extra RF amplification stages indicates that transmission distance of a few hundreds of metres could be achieved with the obtained performance results.

\section{ACKNOWLEDGEMENTS}

This work has been carried out within the FP7 ICT-HELIOS project. Dr. E. Tangdiongga from TU/e is acknowledged for providing the DMZI via the Euro-FOS lab. Dr. D. Moodie from CIP technologies Ltd. and the FP6 ICT-IPHOBAC project are acknowledged for providing the $60 \mathrm{GHz}$ reflective EAM

\section{REFERENCES}

[1] A. Seeds and K. J. Williams, J. Lightwave Technol. 24, 4628 (2006)

[2] J. Capmany et al., Photon. Tech. Lett. 17, 421 (2005)

[3] A. Wiberg et al., J. Lightwave Technol. 25, 2984 (2007)

[4] R. Sambaraju et al., Proc OFC'10, OML1 (2010)

[5] G. K. Gopalkrishnan et al.,IEEE Trans. Microwave Theory Tech., 43, 2318 (1995)

[6] A. H. Gnauck and P. J. Winzer, J. Lightwave Technol. 23, 115 (2005)

[7] www.project-helios.eu 



\section{Radio Frequency Transparent Demodulation for Broadband Wireless Links}

R. Sambaraju, D. Zibar, A. caballero, I. T. Monroy, R. Alemany, and J. Herrera

in Proc. Optical Fiber Communication Conference/National Fiber Optic Engineers Conference, paper OML1, San Diego CA, Mar. 2010. 


\title{
Radio Frequency Transparent Demodulation for Broadband Wireless Links
}

\author{
Rakesh Sambaraju ${ }^{1}$, Darko Zibar ${ }^{2}$, Ruben Alemany ${ }^{1}$ Antonio Caballero ${ }^{2}$, Javier Herrera ${ }^{1}$ and Idelfonso \\ T. Monroy ${ }^{2}$ \\ Valencia Nanophotonics Technol. Center, Universidad Politécnica de Valencia, Camino de Vera s/n, Ed.8F,46022Valencia, Spain. \\ DTU Fotonik, Technical University of Denmark, Oersteds Plads Building 343, DK-2800 Kgs. Lyngby, Denmark. \\ E-mail: rsambaraju@ntc.upv.es
}

\begin{abstract}
Novel demodulation technique for radio over fiber systems, transparent to RF carrier frequency, employing coherent detection and baseband digital signal processing is presented for the first time. Multi-gigabit signal demodulation at $40 \mathrm{GHz}$ RF is demonstrated

(C2009 Optical Society of America
\end{abstract}

OCIS codes: (060.4080) Modulation; (060.5625) Radio frequency photonics

\section{Introduction}

The increase in the data capacity demand is pushing the development of multi-gigabit wireless systems. Currently, there is a lot of research effort underway in order to develop multi-gigabit wireless systems addressing applications like LAN bridging, inter-building communications, mobile backhaul, etc [1-3]. Several frequency bands in the millimeter wave frequency regime $(60 \mathrm{GHz}, 70 / 80 \mathrm{GHz},>100 \mathrm{GHz}$ etc) have a few $\mathrm{GHz}$ of available bandwidth, which could potentially enable gigabit wireless transmissions. However, in order to achieve multi-gigabit capacity in the limited available millimeter wave bandwidth, spectrally efficient modulation formats like QPSK and M-QAM are required. Radio over fiber technologies [4] provide a good solution for such broadband wireless systems and up to $10 \mathrm{~Gb} / \mathrm{s}$ wireless signal generation with both, on-offkeying and spectral efficient M-QAM modulation has been demonstrated [2,3]. However, the detection of these vector modulated multi-gigabit signals using conventional electrical methods becomes complicated when the bit rate increases and carrier frequency approaches millimeter wave frequencies. Recently, many photonic techniques for demodulation of M-QAM signals have been proposed [5, 6], but still require relatively complex high-bandwidth analog phase locked loop. Also, recently novel digital coherent receiver structures have been proposed for radio over fiber links [7,8]. Although, the demonstrated digital coherent techniques do not require electronic or optical phase locked loops, they still require electronics such as A/D converters or signal sources at high RF carrier frequencies [9].

In this paper, we present a novel technique for demodulation of high-frequency multi-gigabit RF signals using coherent detection and baseband digital signal processing. The advantage of the proposed technique is that it is transparent to RF carrier frequency and only requires A/D converters at the baseband/data rate frequency. In order to demonstrate the RF frequency transparency, demodulation of $2.5 \mathrm{~Gb} / \mathrm{s}$ QPSK modulated data signal, at $40 \mathrm{GHz}$ and $35 \mathrm{GHz}$ RF carrier frequency, is experimentally demonstrated.

\section{Experimental set-up}

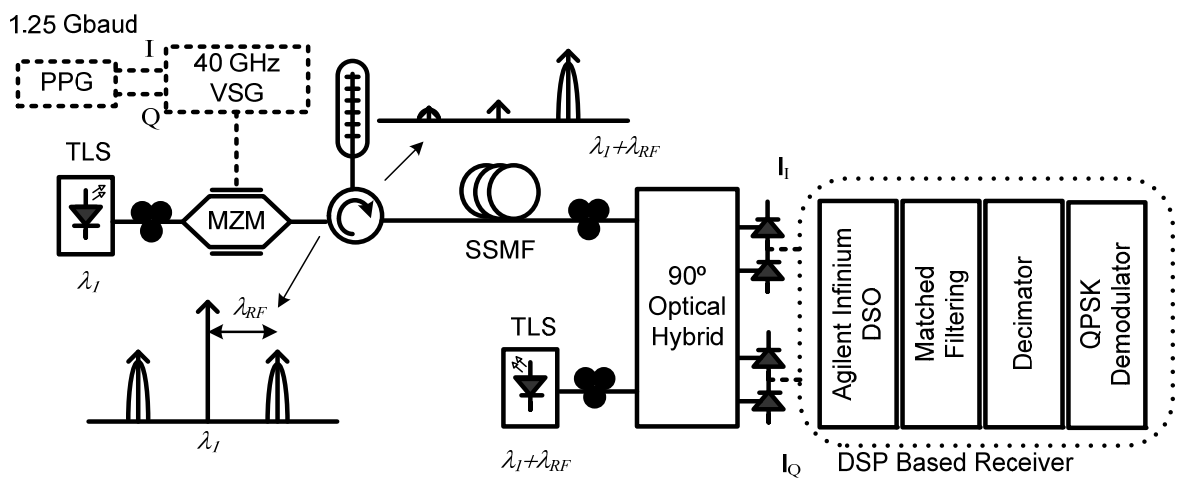

Figure 1: Schematic of the experimental setup of RF transparent demodulator. 
The schematic of the experimental setup is shown in Fig. 1. A pattern generator is used to generate inphase and quadrature components of the 1.25 Gbaud QPSK data signal Thereafter, a vector signal generator is used to upconvert the generated $2.5 \mathrm{~Gb} / \mathrm{s}$ QPSK signals at the RF carrier frequency of $40 \mathrm{GHz}$. The electrical power of the RF signal was set to $+12 \mathrm{dBm}$. An optical carrier at the wavelength of $1554.68 \mathrm{~nm}$ generated from an external cavity tunable laser (TLS) was amplitude modulated, with the 1.25 Gbaud QPSK modulated $40 \mathrm{GHz}$ RF carrier frequency data signal, using a MZ modulator biased at the quadrature bias point. The output of the MZ modulator consists of the optical carrier $(1554.68 \mathrm{~nm})$, and two sidebands, at $1554.36 \mathrm{~nm}$ and $1555.0 \mathrm{~nm}$, each modulated with 1.25 Gbaud QPSK data and separated by $40 \mathrm{GHz} / 0.32 \mathrm{~nm}$. The main idea behind our approach is now to filter out one of the sidebands of the modulated optical carrier and in this way only transmit baseband data making this scheme RF carrier frequency independent, and also dispersion tolerant since only the baseband data is transmitted. This is illustrated in Fig. 1. To filter out the sideband at $1555.0 \mathrm{~nm}$, a fiber Bragg grating (FBG) centered at $1555.0 \mathrm{~nm}$ with an optical bandwidth of $25 \mathrm{GHz}$ is used in combination with an optical circulator. The optical signal at $1555.0 \mathrm{~nm}$ contains purely 1.25 Gbaud QPSK data signal modulation. The optical data signal at $1555.0 \mathrm{~nm}$, carrying $2.5 \mathrm{~Gb} / \mathrm{s}$ of data, is then transmitted over a $26 \mathrm{~km}$ prior to coherent detection, which only requires baseband signal processing at twice the data rate. An LO optical signal at around $1555 \mathrm{~nm}$ with a linewidth of around $100 \mathrm{kHz}$ generated from another tunable external cavity laser is mixed with the filtered sideband in the optical $90^{\circ}$ hybrid. The inphase $I_{i}$ and quadrature $I_{q}$ optical components are detected using a pair of balanced photoreceiver, which were inbuilt in the optical $90^{\circ}$ optical hybrid. The photodetected signals were then digitized using a high bandwidth realtime oscilloscope with $13 \mathrm{GHz}$ analog bandwidth and the sampling rate up to $40 \mathrm{GS} / \mathrm{s}$. The digitized photodetected signals were later used for offline digital signal processing consisting of matched filtering, decimation and QPSK demodulation module [8].

\section{Results}

In Fig. 2(a), optical spectra of the input, transmitted and the reflected signal from the FBG is shown. It is the reflected signal at $1550.0 \mathrm{~nm}$ containing $2.5 \mathrm{~Gb} / \mathrm{s}$ QPSK data modulation that is sent to the transmission span of $26 \mathrm{~km}$ and subsequent signal demodulation. It is observed that the FBG suppresses the optical carrier and sidebands by more that $30 \mathrm{~dB}$, allowing for pure baseband transmission.

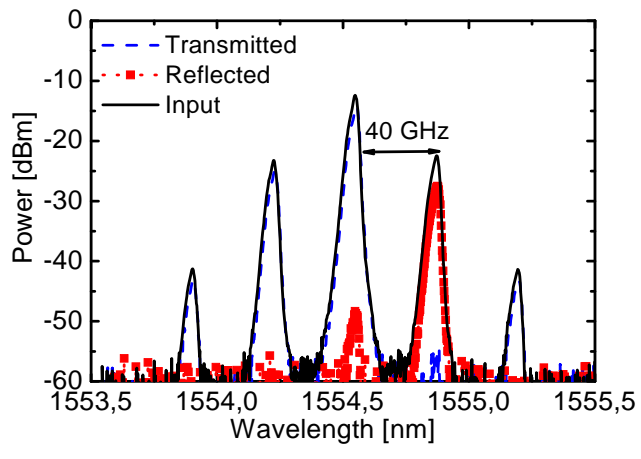

(a)

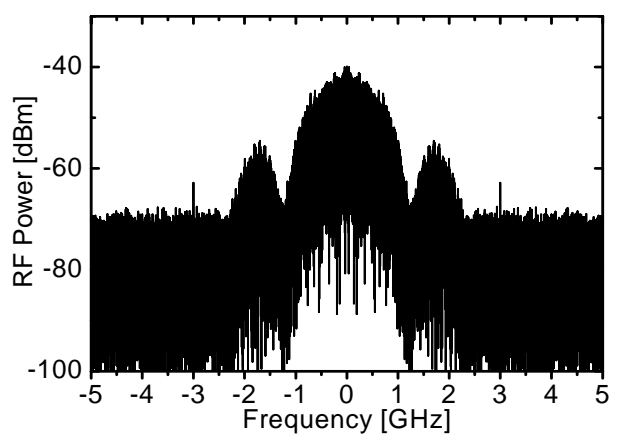

(b)

Fig. 2 (a) Optical spectrum input to, reflected from and transmitted through the fiber Bragg grating (a), the electrical spectrum of the photocurrent output from one of the arms of the $90^{\circ}$ optical hybrid.

In Fig. 2(b), the frequency spectrum of the detected photocurrent (one of the outputs of the $90^{\circ}$ optical hybrid) is shown. It is observed that the signal is in baseband with a small frequency offset from zero due to frequency mismatch between the transmitter and LO laser. This frequency offset is corrected by the digital signal processing based demodulation algorithm [8]. Fig. 3(a) shows the Bit Error Ratio (BER) curves plotted as a function of the Optical Signal to Noise Ratio (OSNR). To begin with, we focus on the data signal at $40 \mathrm{GHz} \mathrm{RF}$ carrier frequency. First, the BER was measured in a back-to-back scenario, and then a $26 \mathrm{~km}$ fiber transmission was performed. Fig. 3(a) shows that for the back-to-back scenario successful signal demodulation is achieved with the receiver sensitivity of $8 \mathrm{~dB}$ in order to obtain the BER of $10^{-4}$. Additionally, it can be observed from Fig. 3(a), that the $26 \mathrm{~km}$ of fiber transmission did not induce any penalty for the demodulation of a $2.5 \mathrm{~Gb} / \mathrm{s}$ QPSK signal at $40 \mathrm{GHz}$ RF carrier, proving the dispersion tolerance of the proposed scheme.

Later, the RF carrier frequency was changed to $35 \mathrm{GHz}$ while still keeping the 1.25 Gbaud QPSK data signal modulation. Changing the RF carrier frequency from $40 \mathrm{GHz}$ to $35 \mathrm{GHz}$, has moved the sideband center 


\section{OML1.pdf}

wavelength, so in order to be able to filter the sideband properly, the transmitter laser was detuned to maximize the filtered optical power. In Fig. 3(a), the BER as a function of OSNR is also plotted when the RF carrier frequency is $35 \mathrm{GHz}$. The error free (zero errors counted) signal demodulation is achieved when the OSNR is 7 dB, which is indicated by an arrow in Fig. 3(a). An improvement in the BER is observed compared to the case when the RF carrier frequency was $40 \mathrm{GHz}$, which is due to the increased frequency response of the MZ modulator at $35 \mathrm{GHz}$ compared to $40 \mathrm{GHz}$. Also a $2 \mathrm{~dB}$ of increase in the sideband power was recorded.

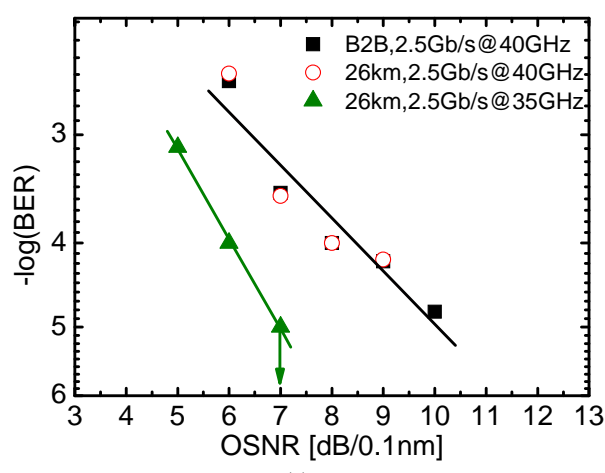

(a)

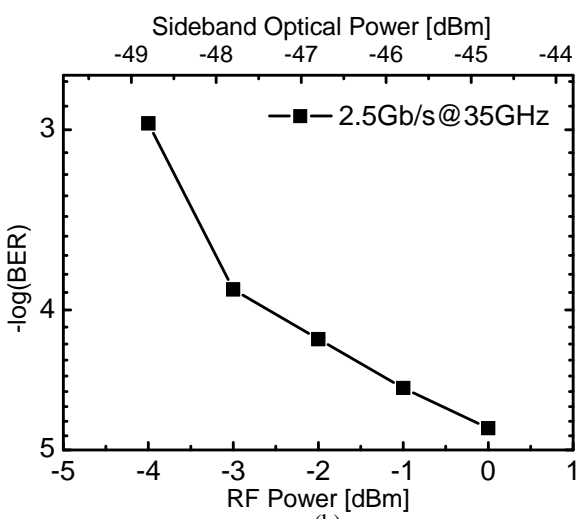

(b)

pFig. 2 The bit error ratio plotted as a function of OSNR (a), and as a function of the electrical RF power input to the MZ modulator.

To analyze the effect of the received RF power, the BER was measured as a function of the RF signal power without any additional optical noise and plotted in Fig. 3(b), for the RF carrier frequency of $35 \mathrm{GHz}$ The RF signal power was varied from $-4 \mathrm{dBm}$ to $0 \mathrm{dBm}$, and the corresponding optical sideband power was also measured and is shown in Fig. 3(b). The Fig. 3(b) shows that even for very low RF signal power of $-4 \mathrm{dBm}$, (sideband optical power of $-48 \mathrm{dBm}$ ), BER of app. $10^{-3}$, is achievable. This proves the high sensitivity of the proposed demodulation scheme.

\section{Conclusion}

A new demodulation technique, transparent to RF carrier frequency based on optical coherent detection and baseband digital signal processing is presented. A successful experimental demodulation of $2.5 \mathrm{~Gb} / \mathrm{s}$ QPSK data signal at $40 \mathrm{GHz}$ RF carrier frequency is successfully demonstrated. To the best of our knowledge, this is the first RF transparent photonic demodulation technique which is dispersion tolerant and does not use any highspeed electrical RF components.

\section{Acknowledgment}

This work has been supported by the European Commission FP7 under Network of Excellence project EUROFOS (22402).

\section{References}

[1] V. Dayadyuk et al, IEEE Microwave Theory and Techniques, vol. 55, no. 12, pp. 2813-2820, 2007.

[2] M. Hirata et al, IEEE/OSA Journal of Lightwave Technology., vol. 21, no. 10, pp. 2145-2153, 2003.

[3] R. Sambaraju et al, Optics Letters, vol. 33, no. 16, pp. 1833-1835, 2008.

[4] J. Capmany and D. Novak, Nature Photonics, vol. 1, pp. 319-330, 2007.

[5] G. K. Gopalakrishnan et al., IEEE Microwave Theory and Techniques., vol. 43, no. 9, pp. 2318-2323, 1995.

[6] R. Sambaraju et al., International Topical Meeting on Microwave Photonics 2008, pp. 117-120, 2008.

[7] T. Clark et al., IEEE Photonics Technology Letters, vol. 19, no. 16, pp. 1206 - 1208, 2009

[8] D. Zibar et al., IEEE Photonics Technology Letters, vol. 21, no. 3, pp. 155-157, 2009

[9] A. Caballero et al., in Proc. European Conf. on Optical Communications, PD3.3, pp 58-59, 2009. 


\section{Paper L}

$100 \mathrm{GHz}$ Wireless-over-Fibre Links With up to $16 \mathrm{~Gb} / \mathrm{s}$ QPSK Modulation using Optical Heterodyne Generation and Digital Coherent Detection.

R. Sambaraju, D. Zibar, A. caballero, I. T. Monroy, R. Alemany, and J. Herrera

in IEEE Photonics Technology Letters, Vol. 22, No. 22, pp. 1650-1653, 2010 . 


\title{
100-GHz Wireless-Over-Fiber Links With Up to 16-Gb/s QPSK Modulation Using Optical Heterodyne Generation and Digital Coherent Detection
}

\author{
Rakesh Sambaraju, Darko Zibar, Antonio Caballero, Idelfonso T. Monroy, Ruben Alemany, and Javier Herrera
}

\begin{abstract}
In this letter, a novel technique for direct conversion of an optical baseband quadrature phase-shift keying (QPSK) signal to a millimeter-wave wireless signal and subsequent signal demodulation is reported. Optical heterodyne mixing of the optical baseband QPSK signal with a free-running unmodulated laser for the wireless signal generation is employed. To correct for the phase and frequency offset originating from the heterodyne mixing of the two free-running lasers, wireless signal demodulation based on optical coherent detection in combination with baseband digital signal processing is implemented. As a proof of concept, $5-\mathrm{Gb} / \mathrm{s}$ amplitude-shift keying and up to a 16-Gb/s QPSK wireless signal in the band of 75-110 GHz was generated and successfully demodulated. All-photonic millimeter-wave wireless signal generation and digital coherent detection at baud-rate are employed without complex optical phase-locked loop.
\end{abstract}

Index Terms-Coherent detection, digital signal processing, microwave photonics, wireless communication.

\section{INTRODUCTION}

$\mathbf{O}$ PTICAL communication systems are going through a radical change with the return of coherent detection in combination with digital signal processing. This allows for spectrally efficient modulation formats, such as quadrature phase-shift keying (QPSK) or quadrature amplitude modulation ( $M$-QAM), to be used to generate high-speed data signals with the bit rates of up to $112 \mathrm{~Gb} / \mathrm{s}$ while keeping the baud rates at either 10 or 28 Gbaud [1]. The digital signal processing assisted optical coherent detection is maturing very fast [2], and is expected to enter the commercial applications within a few years. On the other hand, wireless technology is also advancing at a rapid pace, especially in the millimeter-wave frequency bands like $60,70 / 80$, and $>100 \mathrm{GHz}$, where several gigahertz of bandwidth are available [3]. Wireless links with capacities of $10 \mathrm{~Gb} / \mathrm{s}$, and even up to $28 \mathrm{~Gb} / \mathrm{s}$ have been demonstrated, using microwave photonic techniques [4]. However, the current technologies for generation of high-capacity wireless signals

Manuscript received June 08, 2010; revised July 23, 2010; accepted September 03, 2010. Date of publication September 16, 2010; date of current version October 22, 2010. This work has been supported by the European Commission FP7 under Network of Excellence project EUROFOS (22402) www.eurofos.eu.

R. Sambaraju, R. Alemany, and J. Herrera are with the Universidad Polítecnica de Valencia, 46022 Valencia, Spain (e-mail: r.sambaraju@ieee.org; rualser@hotmail.com; jaherllo@ntc.upv.es).

D. Zibar, A. Caballero, and I. T. Monroy are with the DTU Fotonik, Tecnical University of Denmark, 2800 Kgs-Lyngby, Denmark (e-mail: dazi@fotonik.dtu.dk; acaj@fotonik.dtu.dk; idtm@fotonik.dtu.dk).

Color versions of one or more of the figures in this letter are available online at http://ieeexplore.ieee.org.

Digital Object Identifier 10.1109/LPT.2010.2076801 either employ spectrally inefficient on-off-keying modulation which are limited to $10 \mathrm{~Gb} / \mathrm{s}$ [5], or use complex electronic arbitrary waveform generators [4].

In this letter, we report for the first time, a novel technique for generation and demodulation of spectrally efficient QPSK wireless signals in the 75- to $110-\mathrm{GHz}$ band. The wireless QPSK signal generation is obtained by directly converting an optical baseband signal into an millimeter-wave wireless signal as explained next. The proposed wireless signal generation is based on heterodyne mixing of a high-speed optical (baseband) QPSK signal with a free-running continuous-wave $(\mathrm{CW})$ laser in a photodiode. The subsequent wireless signal demodulation is based on optical single sideband filtering, coherent detection and baseband digital signal processing, i.e., radio-frequency (RF) transparent signal demodulation [6]. We experimentally demonstrate a successful generation and demodulation of $5-\mathrm{Gb} / \mathrm{s}$ amplitude shift keying (ASK) and up to 16-Gb/s QPSK wireless signals in the $75-$ to $110-\mathrm{GHz}$ frequency band. The proposed technique does not incorporate any millimeter-wave components for the wireless signal generation or detection, and digital coherent detection with electronic components only at twice the data signal baud rate are used. The proposed technique is also highly scalable and does not require any kind of optical phase-locking techniques for the wireless signal generation [7], and detection. The phase and frequency drift originating from wireless signal generation and coherent detection, both employing freerunning lasers, are fully compensated using digital signal processing based detection. This concept significantly simplifies the system complexity and achieves the breakthrough demanded from simple and seamless integration between high-capacity optical fiber links and wireless connections.

\section{EXPERIMENTAL SETUP}

Fig. 1 shows the schematic of the experimental setup for the generation and detection of QPSK wireless signals. In order to generate an optical data signal, a $\mathrm{CW}$ external cavity laser (ECL) at wavelength $\lambda_{1}(1555 \mathrm{~nm})$ is used. The output of the ECL is then phase-modulated by two data streams $I_{k}$ and $Q_{k}$ using a nested dual-parallel Mach-Zehnder modulator (MZM) structure (in-phase/quadrature (I/Q) modulator) to generate an optical baseband QPSK signal. The optical I/Q modulator is driven with up to 8-Gb/s electrical data signals from a bit pattern generator with a PRBS (pseudorandom bit sequence) pattern of length $2^{15}-1$ to generate $16-\mathrm{Gb} / \mathrm{s}$ optical baseband QPSK signals. The optical baseband signal is then amplified, and filtered using a 1-nm bandwidth optical bandpass filter (OBPF) to remove the out-of-band amplified spontaneous emission (ASE) 


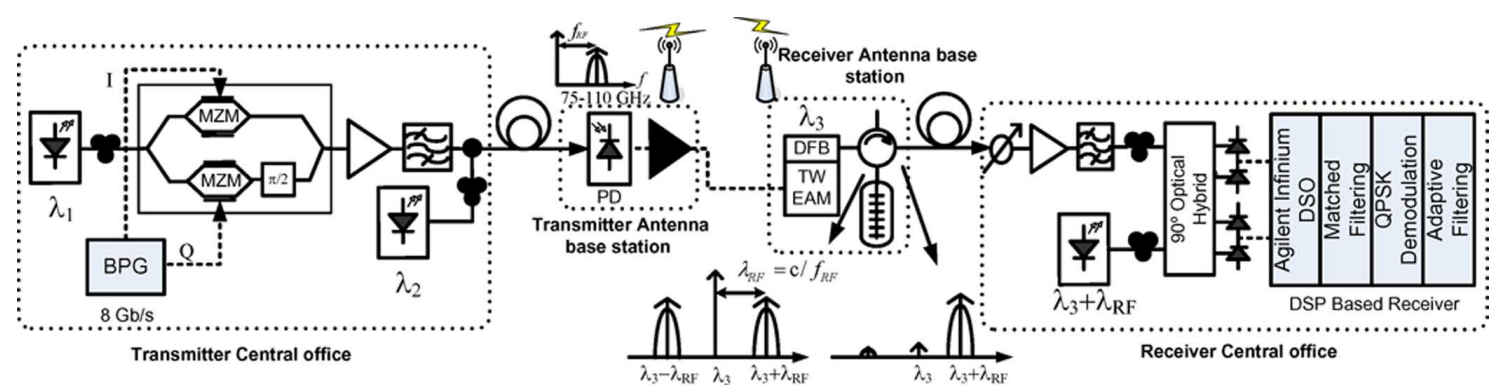

Fig. 1. Experimental setup of a QPSK wireless-over-fiber link. (Antenna at the transmitter and receiver are just for illustration purposes; no wireless transmission is performed).

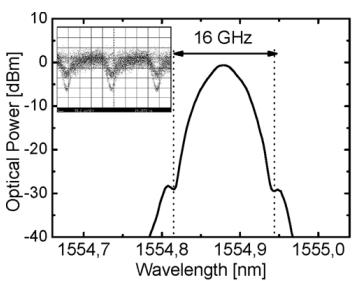

(a)

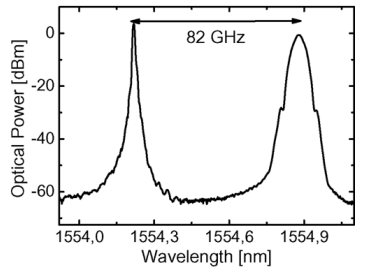

(b)
Fig. 2. (a) Optical spectrum of a 16-Gb/s baseband QPSK signal with the direct detected eye diagram (inset). (b) Optical spectrum of the combined optical baseband 16 QPSK signal and an unmodulated optical carrier with a frequency separation of $\Delta f=f_{\mathrm{RF}}=82 \mathrm{GHz}$.

noise. The optical spectrum of the 16-Gb/s optical QPSK signal is plotted in Fig. 2(a) together with the directly detected eye diagram of the optical QPSK signal [inset Fig. 2(a)]. The eye diagram shows the characteristic intensity dips of a QPSK signal when generated using a nested I/Q MZM. Prior to the up-conversion to the RF carrier frequency, the baseband optical QPSK signal is combined in an optical coupler with another CW optical signal at wavelength $\lambda_{2}(1554.32 \mathrm{~nm})$ emitted from an ECL (see Fig. 1). The frequency difference between the two optical sources, $\Delta f=f_{\mathrm{RF}}$, is chosen to the desired RF carrier frequency. The optical spectrum of the combined optical signals is plotted in Fig. 2(b). The combined optical signals are then transmitted, using 50-m-long single-mode fiber (SMF), to a remote transmitter antenna site (see Fig. 1). At the remote transmitter antenna site, the two optical carriers are heterodyne mixed in a $100-\mathrm{GHz}$ bandwidth photodiode, generating a 16-Gb/s QPSK modulated radio signal at an RF carrier frequency of $f_{\mathrm{RF}}=$ $82 \mathrm{GHz}$. The wireless signal at $82 \mathrm{GHz}$ with $16-\mathrm{Gb} / \mathrm{s}$ QPSK data modulation is then amplified using a $W$-band amplifier (from Radiometer Physics $\mathrm{GmbH}$ ). At the reception point (receiver antenna base station, see Fig. 1), the wireless QPSK signal modulates an optical carrier at wavelength $\lambda_{3}(1549.34 \mathrm{~nm})$ emitted from a distributed-feedback laser integrated with a $100-\mathrm{GHz}$ travelling wave electroabsorption modulator (DFB TW-EAM), developed in the European Project ICT-HECTO. The optical output of the TW-EAM contains a central carrier at $\lambda_{3}$ and the two sidebands at wavelengths $\lambda_{3} \pm \lambda_{\mathrm{RF}}$ (see Fig. 1), where $\lambda_{\mathrm{RF}}=c / f_{\mathrm{RF}}$ and $c$ is the velocity of light. The sidebands $\lambda_{3} \pm \lambda_{\mathrm{RF}}$ are separated by $f_{\mathrm{RF}}=82 \mathrm{GHz}$ from the optical carrier at $\lambda_{3}$, and contain the QPSK data modulation. Using a fiber Bragg grating (FBG) with $>25 \mathrm{~dB}$ of rejection and centered at $1550 \mathrm{~nm}$, one of the sidebands is filtered out such that only pure

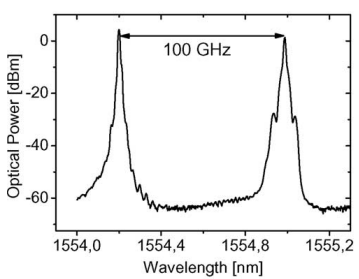

(a)

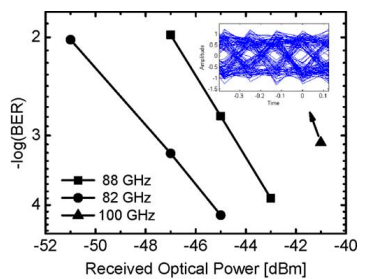

(b)
Fig. 3. (a) Optical spectrum showing the $5-\mathrm{Gb} / \mathrm{s}$ ASK optical data signal and an unmodulated optical carrier prior to the RF up-conversion. (b) BER plots versus the received optical power of the wireless $5-\mathrm{Gb} / \mathrm{s}$ ASK signals at RF frequencies of 82,88 , and $100 \mathrm{GHz}$.

baseband QPSK signal at $\lambda_{3}+\lambda_{\mathrm{RF}}(1550 \mathrm{~nm})$ is sent to the coherent receiver at the receiver central station, after $50 \mathrm{~m}$ of transmission. Prior to demodulation, the baseband QPSK signal is amplified using an optical preamplified receiver, and intradyne mixed in a optical $90^{\circ}$ hybrid with an optical local oscillator emitting from an ECL with a linewidth of $100 \mathrm{kHz}$, and tuned to $\lambda_{3} \pm \lambda_{\mathrm{RF}}$. The photodetected I and Q signals are then sampled at a rate of $20 \mathrm{GS} / \mathrm{s}$ using a real-time digital sampling scope, and subsequently off-line processed using a digital signal processing-based receiver [6].

\section{RESULTS}

The system performance is tested by first setting the system baud rate to 5 Gbaud and applying an ASK modulation format for the following RF carrier frequencies: 82, 88, and $100 \mathrm{GHz}$. Fig. 3(a) shows the optical spectrum at the input of the photodiode, of the optical baseband 5-Gb/s ASK signal together with an unmodulated $\mathrm{CW}$ optical carrier $100 \mathrm{GHz}$ away in frequency, prior to wireless signal generation. At the receiver (receiver central office), the ASK wireless signals were successfully demodulated using the DSP-based receiver and the bit-error ratio (BER) is plotted versus the received optical power, as shown in Fig. 3(b). The received optical power to the preamplified receiver is varied using the variable optical attenuator (VOA) present in the receiver central office (see Fig. 1). Power penalty is observed as the RF carrier frequency increases, which is due to the bandwidth limitation of the RF components. Later the modulation format was changed to QPSK, resulting in a $10-\mathrm{Gb} / \mathrm{s}$ QPSK wireless signal at a carrier frequency of $82 \mathrm{GHz}$. At the receiver, the 10-Gb/s QPSK wireless signal is successfully demodulated with BER values below $10^{-3}$, as shown in Fig. 4. Using digital signal processing, demodulation of the $10-\mathrm{Gb} / \mathrm{s}$ 


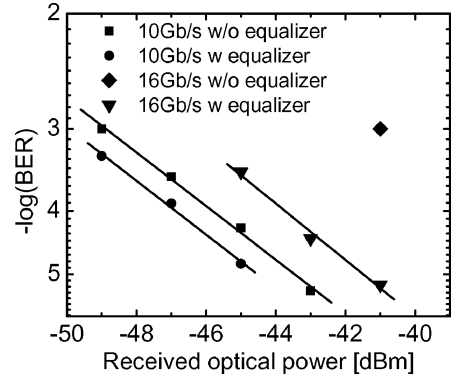

Fig. 4. BER as a function of the received optical power of a 10- and 16-Gb/s wireless QPSK signal at the RF carrier frequency of $82 \mathrm{GHz}$, with and without adaptive equalization.

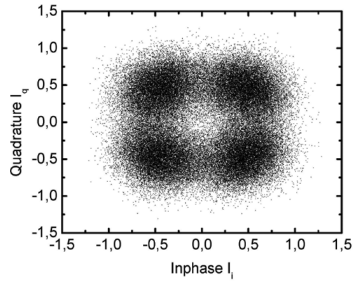

(a)

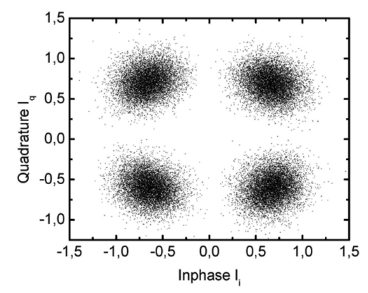

(b)
Fig. 5. Constellation diagrams of a $16-\mathrm{Gb} / \mathrm{s}$ 82-GHz wireless signal. (a) Without and (b) with baud rate adaptive equalizer.

QPSK signals is performed with and without adaptive equalizer at baud rate, and relatively small improvement of around $1 \mathrm{~dB}$ is observed in the performance indicating that no severe inter symbol interference (ISI) due the bandwidth limitations of the $\mathrm{RF}$ components is experienced. Then, the baud rate is changed to 8 Gbaud resulting in a $16-\mathrm{Gb} / \mathrm{s}$ QPSK wireless signal, and the BER of the 16-Gb/s signals is also plotted versus the received optical power in Fig. 4. It is observed that for 8-Gbaud case, the adaptive equalizer pushes down the BER from $10^{-3}$ to $10^{-5}$ for a received optical power of $-41 \mathrm{dBm}$ indicating strong ISI arising from the bandwidth limitations of the RF components. It can also be noted that when changing the bit rate from 10 to $16 \mathrm{~Gb} / \mathrm{s}$ and by using adaptive equalization, a penalty of around $3 \mathrm{~dB}$ is induced, which is mainly attributed to the frequency response of the RF components. For instance, the photodiode has a 3 -dB cut-off at $90 \mathrm{GHz}$, and the $W$-band amplifier has a 2-dB lower gain at $90 \mathrm{GHz}$. In order to demonstrate the effect of adaptive digital equalization, the constellation diagrams of the demodulated QPSK signal with and without digital equalization for $P_{\text {rec }}=-43 \mathrm{dBm}$ are plotted in Fig. 5(a) and (b), respectively. It is observed from Fig. 5(a), where digital equalization is not applied, that it is very difficult to distinguish data points and no successful demodulation can be performed. After applying the equalization [see Fig. 5(b)], the constellation points look clear and well separated with successful signal demodulation at a BER below $10^{-4}$. The applied equalizer has only seven taps and can be thereby easily implemented in hardware. Apart from the bandwidth limitations of the RF components, the critical parameter for the system performance is the amount of the total frequency offset which can be tracked by the digital carrier-recovery scheme. For the wireless signal generation and subsequent detection, four different lasers are used in total, therefore, the frequency offset may be large. In general, the ratio between the total frequency offset and the baud rate should not exceed $10 \%$, otherwise, advanced frequency tracking algorithms must be employed. Another issue is that the total amount of phase noise, which may set the limitations for the choice of the employed modulation format.

\section{CONCLUSION}

Using digital coherent detection for wireless signal demodulation, all-optical generation of multigigabit-per-second spectrally efficient wireless signals can be obtained by simple heterodyne mixing of an optical baseband signal with another free-running laser in a photodiode. Successful wireless signal generation and demodulation of a 5-Gb/s ASK and 16-Gb/s QPSK wireless signal in the 75- to $110-\mathrm{GHz}$ band is performed demonstrating the modulation format transparency. The proposed system does not involve any millimeter-wave components (only electronics at twice the baud rate are employed) or any high-frequency analogue phase-locking mechanism, resulting in the reduced overall system complexity.

\section{ACKNOWLEDGMENT}

The authors acknowledge U. Westergren of the project ICT-HECTO (www.hecto.eu) for providing the $100-\mathrm{Gb} / \mathrm{s}$ DFB-TWEAM; A. Walber from Radiometer Physics $\mathrm{GmbH}$ for providing the 100-GHz amplifier; D. Gonzalo from Agilent Spain, Vedran Furtula DTU • Electro and S. Bojanic from Anritsu Denmark.

\section{REFERENCES}

[1] P. Winzer, A. Gnauck, C. Doerr, M. Magarini, and L. Buhl, "Spectrally efficient long-haul optical networking using $112-\mathrm{Gb} / \mathrm{s}$ polarization-multiplexed 16-QAM," J. Lightw. Technol., vol. 28, no. 4, pp. 547-556, Feb. 1, 2010.

[2] C. R. Doerr, L. Zhang, and P. J. Winzer, "Monolithic InP multi-wavelength coherent receiver," in Proc. Optical Fiber Communication Conf. (OFC'10), San Diego, CA, 2010, Paper PDPB1.

[3] J. A. Wells, "Faster than fiber: The future of multi-G/s wireless," IEEE Microw. Mag., vol. 10, no. 3, pp. 104-112, May 2009.

[4] C.-T. Lin, E.-Z. Wong, W.-J. Jiang, P.-T. Shin, J. Chen, and S. Chi, "28-Gb/s 16-QAM OFDM radio-over-fiber system within 7-GHz license-free band at $60 \mathrm{GHz}$ employing all-optical up-conversion," in Proc. Conf. Lasers and Electro-Optics/Quantum Electronics and Laser Science (CLEO/QELS 2009), 2009, Paper CPDA8.

[5] R. W. Ridgway and D. W. Nippa, "Generation and modulation of a 94-GHz signal using electrooptic modulators," IEEE Photon. Technol. Lett., vol. 20, no. 8, pp. 653-655, Apr. 15, 2008.

[6] R. Sambaraju, D. Zibar, R. Alemany, A. Caballero, and J. Herrera, "Radio frequency transparent demodulation for broadband wireless links," in Proc. Optical Fiber Communication Conf. (OFC'10), San Diego, CA, 2010, Paper OML1.

[7] L. A. Johansson and A. J. Seeds, "36-GHz 140-Mb/s radio-over-fiber transmission using an optical injection phase-lock loop source," IEEE Photon. Technol. Lett., vol. 13, no. 8, pp. 893-895, Aug. 2001. 


\section{Paper M}

\section{Up to $40 \mathrm{~Gb} / \mathrm{s}$ Wireless Signal Generation and Demodulation in 75-110 GHz Band using Pho- tonic Techniques}

R. Sambaraju, D. Zibar, A. caballero, J. Herrera, J. B. Jensen, I. T.

Monroy, U. Westergren, A. Walber, and J. Martí

in 2010 International Topical Meeting on Microwave Photonics, MWP2010, postdeadline paper. Oct. 2010. 


\section{Up to $40 \mathrm{~Gb} / \mathrm{s}$ Wireless Signal Generation and Demodulation in 75-110 GHz Band using Photonic Techniques}

\section{Rakesh Sambaraju, Javier Herrera, Javier Marti}

Valencia Nanophotonics Technology Center,

Univ. Politecnica de Valencia, Camino de Vera s/n, Valencia 46022, Spain. E-mail: r.sambaraju@ieee.org

\section{Urban Westergren}

Royal Institute of Technology, KTH, Photonics and Microwave Eng. 229 SE-164 40 Kista, Sweden

\begin{abstract}
Record wireless signal capacity of up to $40 \mathrm{~Gb} / \mathrm{s}$ is demonstrated in the 75-110 GHz band. All-optical OFDM and photonic up-conversion are used for generation and digital coherent detection for demodulation.
\end{abstract}

\section{INTRODUCTION}

Wireless links that can provide the same capacity as optical communication systems will provide a cost-effective solution for future's wireless/wireline seamless network integration [1]. To realize high capacity wireless links approaching 40 $\mathrm{Gb} / \mathrm{s}$ and beyond, efficient technologies to generate and demodulate spectrally efficient modulation formats at mmwave frequency are mandatory. The current trends in achieving record wireless capacities is by using electrical Orthogonal Frequency Division Multiplexing (OFDM) and using optical modulators for up-conversion to the desired RF carrier frequency $[2,3]$. The techniques presented in $[2,3]$ have severe limitations: they require high-bandwidth complex arbitrary waveform generators and high-frequency RF components for signal generation and demodulation. Scaling those approaches to $40 \mathrm{~Gb} / \mathrm{s}$, and beyond, at mm-wave frequencies, is very challenging.
Darko Zibar, Antonio Caballero, Jesper B. Jensen,
Idelfonso, T. Monroy

DTU Fotonik, Dept. of Photonic Engineering, Technical Univ. of Denmark, Ørsteds Plads, byg. 343, DK 2800 Kgs.

Lyngby, Denmark Email: dazi@fotonik.dtu.dk

Achim Walber

Radiometer Physics GmbH, Birkenmaarstraße 10, 53340 Meckenheim Germany

What is most important is that in a converged wireless/wireline network scenario, seamless integration between the optical and wireless signal formats with RF/bitrate transparency and scalability is crucial. This is currently not achievable using conventional approaches [2, 3]. In this paper, we present a novel concept for high-capacity wireless links, based on all-optical OFDM and transparent RF carrier generation. The proposed technique relies on seamlessly converting a high-capacity all-optical OFDM baseband signal to a desired RF carrier frequency, without changing its modulation format, as shown in Fig. 1. This property provides complete transparency for integration between wireline and wireless signalling even at higher networking layers. In our experimental demonstration, signal generation is performed by heterodyne mixing of an all-optical OFDMQPSK [4] modulated baseband signal with another freerunning optical carrier to generate a high capacity mm-wave signal. The RF transparent signal demodulation, based on digital coherent detection [5] is used at the receiver. To demonstrate the scalability of the proposed, system, mmwave wireless signals at different baud-rates and with various numbers of OFDM subcarriers were generated at both the 60

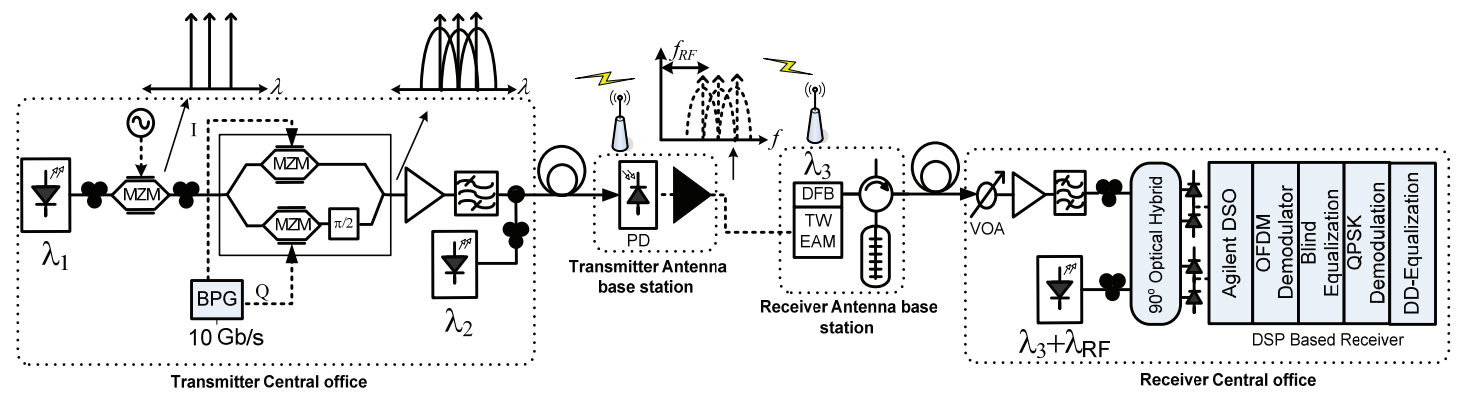

Figure 1: Experimental setup for the generation and demodulation of OFDM wireless signals. (The antennas are only for illustrative purpose and no wireless transmission was performed.) BPG: bit pattern generator, VOA: variable optical attenuator. 
$\mathrm{GHz}$ band and the 75-110 GHz band. Using this approach, we successfully generate and demodulate up to $40 \mathrm{~Gb} / \mathrm{s}$ wireless signals in the $75-110 \mathrm{GHz}$ band. To the best of our knowledge this is the highest wireless capacity reported. Free-running lasers operation for heterodyne generation and detection without the need of any optical phase locked loops is achieved. The data demodulation and advanced signal equalization are performed in software resulting in significant complexity reduction and increased flexibility.

\section{EXPERIMENTAL SET-UP}

The experimental set-up for the generation and detection of high-capacity wireless OFDM signals is shown in Fig. 1. First, a high-capacity all-optical OFDM signal is generated. In the experiment, we generate up to three subcarriers alloptical OFDM signals. For the generation of two subcarriers all-optical OFDM signal, an optical carrier emitted from an external cavity laser (ECL, $\lambda_{1}=1549.8 \mathrm{~nm}$ ) is modulated by a sinusoidal electrical clock signal at frequency half the baud rate, in a MZM biased at its minimum transmission point. The minimum transmission point generates an Optical Carrier Suppression (OCS) modulation where the two OFDM subcarriers separated by the baud rate are generated. Similarly, for generating three subcarriers all-optical OFDM signal, the electrical clock signal frequency is changed to the baud rate and the MZM bias is chosen by optimizing the power of the three subcarriers (the optical carrier, and the sidebands). The optical subcarriers are then fed into an optical I/Q modulator, where two independent data streams (pseudo random bit sequence of length $2^{15}-1$ ) at baud rate modulate the phase of the subcarriers resulting in QPSK modulation on the subcarriers. The output of the optical I/Q modulator is an all-optical OFDM-QPSK modulated signal. The all-optical OFDM-QPSK modulated signal is then amplified and combined with another un-modulated $\mathrm{CW}$ optical carrier $\left(\lambda_{2}\right)$.The combined all-optical OFDM-QPSK signals and the un-modulated $\mathrm{CW}$ carrier are then transmitted to the remote antenna site (see Fig. 1) where they are heterodyne mixed in a (100 GHz bandwidth) Photo-Diode (PD). The polarization of the optical signals is optimized prior to photo-detection to maximize the RF power at the output of the PD. The output of the photo-diode is a highcapacity OFDM-QPSK electrical signal at the desired RF carrier frequency. The desired RF carrier frequency is simply chosen by varying the wavelength of the un-modulated $\mathrm{CW}$ optical source.

The electrical mm-wave OFDM-QPSK modulated signal is received at the receiver antenna site (Fig. 1) and transmitted through a few meter of fibre $(\sim 20 \mathrm{~m})$ prior to signal demodulation using the RF transparent technique [5]. Prior to demodulation, the mm-wave signal is electrically amplified and modulated on an optical carrier at $\lambda_{3}(1549.2 \mathrm{~nm})$, emitted from a distributed feed-back laser integrated with a $100 \mathrm{GHz}$ travelling wave electro-absorption modulator (DFBTW-EAM), and developed in the European Project ICT-
HECTO. Fig. 2 shows the output of the TW-EAM when modulated using a 10 Gbaud two subcarrier OFDM-QPSK wireless signal in the $75-110 \mathrm{GHz}$ band $\left(f_{R F}=82 \mathrm{GHz}\right)$. From Fig. 2, it can be seen that the sidebands $82 \mathrm{GHz}$ apart from the optical carrier $\left(\lambda_{3}\right)$ contain the two subcarrier OFDMQPSK signal. To perform the demodulation, only one of the sidebands is required, which is filtered out using a fibre Bragg's grating (FBG extinction ratio $>25 \mathrm{~dB}$ ).

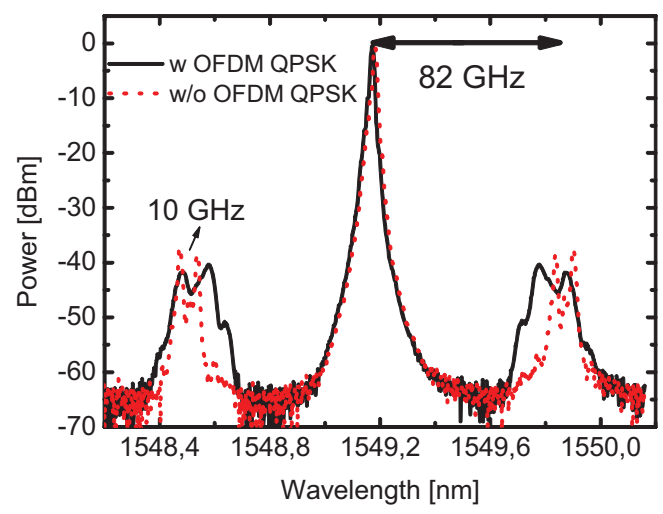

Figure 2: The optical spectrum at the output of the TW-EAM with and without the OFDM QPSK data modulation on the $75-110 \mathrm{GHz}$ band wireless signal.

The filtered sideband is fed into an optical pre-amplified receiver prior to detection. The pre-amplified optical signals are intradyne mixed with an optical local oscillator (LO) in an optical $90^{\circ}$ hybrid. The photo-detected in-phase and quadrature outputs of the optical hybrid are sampled using a $20 \mathrm{GS} / \mathrm{s}$ real-time oscilloscope and demodulated offline. The demodulation process consists of OFDM demodulation, blind equalization, QPSK demodulation and Decision-Directed (DD) post-equalization.

\section{RESULTS}

In order to test the scalability to the RF carrier frequency and bit rate, the system is tested in $60 \mathrm{GHz}$ and $75-110 \mathrm{GHz}$ band. First, the baud rate is set to 5 Gbaud, and the RF carrier frequency is chosen to $60 \mathrm{GHz}$. In, Fig. 3, the bit error rates, versus the received optical power, of the single carrier QPSK and two subcarriers OFDM-QPSK wireless signal are plotted. It is observed that for the single carrier and multi-carrier OFDM modulation format, the BER below $10^{-3}$, is achieved indicating successful signal demodulation. Additionally, the results indicate that it is possible to use all-optical OFDM for high-capacity wireless signal generation.

By moving from $60 \mathrm{GHz}$ to $75-110 \mathrm{GHz}$ band, we have more available bandwidth and the baud rate is therefore increased from 5 Gbaud to 10 Gbaud, resulting in the total bit rate of 20 $\mathrm{Gb} / \mathrm{s}$. A power penalty of around $4 \mathrm{~dB}$ is observed in the case of single carrier QPSK modulation at 10 Gbaud for the BER of $10^{-3}$. 


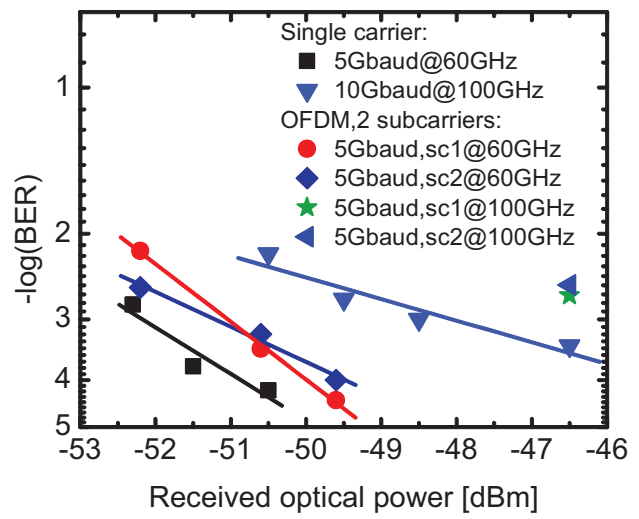

Figure 3: The BER plotted as a function of received optical power for single carrier and multi-carrier OFDM in $60 \mathrm{GHz}$ and $75 \mathrm{GHz}-110 \mathrm{GHz}$ band.

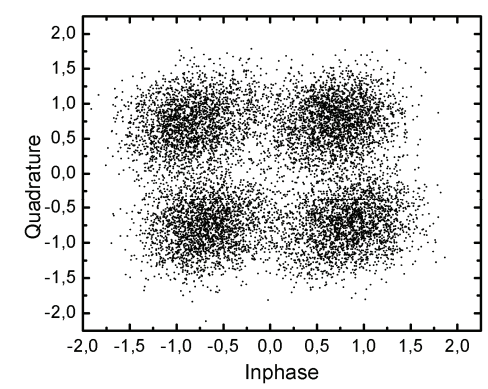

(a)

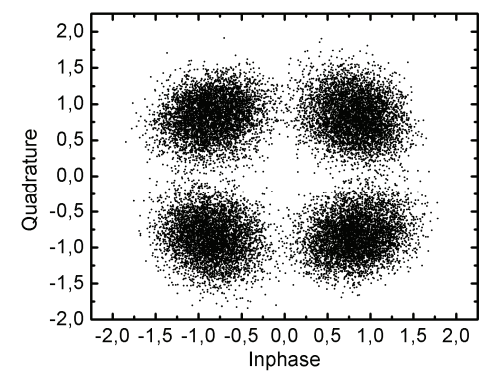

(b)

Figure 4: Constellation diagrams of the $40 \mathrm{~Gb} / \mathrm{s} 2$ subcarrier OFDM-QPSK wireless signals. (a) Subcarrier 1, (b) Subcarrier 2.

This power penalty is as expected, since the amplifier used in the $75-110 \mathrm{GHz}$ band has around $6 \mathrm{~dB}$ lower gain compared to the one used at $60 \mathrm{GHz}$. Similarly the $100 \mathrm{GHz}$ photodiode used for both $60 \mathrm{GHz}$ and $75-110 \mathrm{GHz}$ band has a $3 \mathrm{~dB}$ cut off bandwidth around $90 \mathrm{GHz}$. Also, the TW-EAM has a cut-off bandwidth of $75 \mathrm{GHz}$, and the product of the frequency response of all these components severely distorts the $75-110 \mathrm{GHz}$ signals. We also tested the wireless signal generation and demodulation using two subcarriers all-optical OFDM at 5 Gbaud in $75-110 \mathrm{GHz}$ band. The BER for the received power of $-46.5 \mathrm{dBm}$, is shown in Fig. 3, and a relatively large penalty is observed compared to the single carrier 10 Gbaud case.

Even though the two subcarriers OFDM-QPSK signal at 5 Gbaud occupies less bandwidth compared to the single carrier at 10 Gbaud, the bandwidth limitation of the components and nonlinear phase response is more crucial for OFDM signal. Bandwidth limitation and phase ripple will destroy the orthogonality among the subcarriers and introduce ISI which is difficult to get rid of.

\section{$40 \mathrm{~Gb} / \mathrm{s}$ OFDM Signal Generation}

For the generation of $40 \mathrm{~Gb} / \mathrm{s}$ wireless signals the baud rate was set to $10 \mathrm{GBaud}$ which results in $20 \mathrm{~Gb} / \mathrm{s}$ data rate with single carrier QPSK modulation. Two sub carriers were generated in an OFDM configuration resulting in a $40 \mathrm{~Gb} / \mathrm{s}$ optical 2 sub carrier OFDM-QPSK signal. The heterodyne mixing and photo detection results in a $40 \mathrm{~Gb} / \mathrm{s}$ wireless signal. The constellation diagrams of both the subcarriers of a 2-subcarrier OFDM-QPSK signal in the 75-110 GHz band are plotted in Fig. 4. Fig.4, indicates that in spite of the sever bandwidth limitations, constellation diagrams can be recovered. The BER of the subcarrier 1 and subcarrier 2 is 1.8 and -2.7 (below UFEC), respectively. It can be seen that the subcarrier 1 has a distorted constellation compared to the other subcarrier. This can be explained from the bandwidth limitations of the RF components involved. The total electrical bandwidth of the $40 \mathrm{~Gb} / \mathrm{s}$ QPSK signal is around 25 $\mathrm{GHz}(70-95 \mathrm{GHz})$, which is more than the bandwidth of the RF components, which severely distort the OFDM signal.

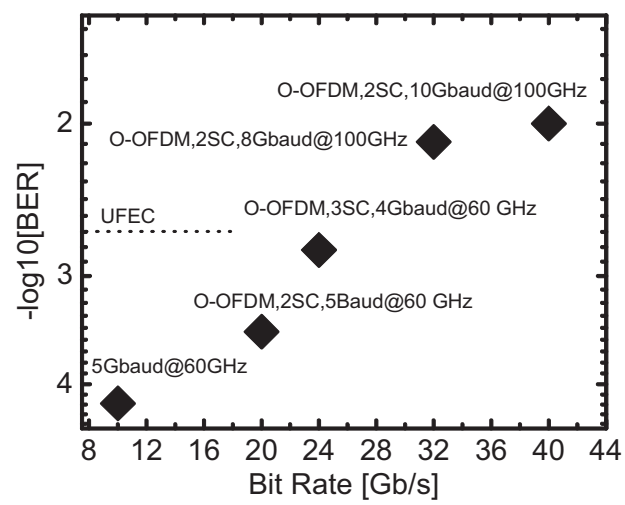

Figure 5: The BER as a function of generated bit-rate for all-optical OFDMQPSK wireless signal generation in $60 \mathrm{GHz}$ and $75 \mathrm{GHz}-110 \mathrm{GHz}$ band.

In Fig. 5, we have summarized the highest achieved bit-rates when all-optical OFDM is used for wireless signal generation in $60 \mathrm{GHz}$ and $75-110 \mathrm{GHz}$ band. At $60 \mathrm{GHz}$, we push the 
system performance by employing three subcarriers alloptical OFDM-QPSK for wireless signal generation. Taking into consideration the bandwidth limitations of components, the baud rate is set to $4 \mathrm{GBaud}$, resulting in a total bit rate of $24 \mathrm{~Gb} / \mathrm{s}$. It is observed in Fig. 4, that the average BER of the three subcarriers is below the UFEC limit. In the $75 \mathrm{GHz}-110$ $\mathrm{GHz}$ band, the baud rate is first increased to $8 \mathrm{GBaud}$ and two subcarriers all-optical OFDM is used for wireless signal generation resulting in the total bit rate of $32 \mathrm{~Gb} / \mathrm{s}$. Next, the baud rate is increased to 10 GBaud, resulting in the total bit rate of $40 \mathrm{~Gb} / \mathrm{s}$ by applying two subcarriers OFDM-QPSK, see Fig. 4, as explained earlier.

\section{CONCLUSIONS}

We have presented a novel scalable high-capacity wireless signal generation technique based on a conversion of an alloptical OFDM baseband singnal, to the wireless signal, by simple heterodyning and digital coherent detection at the receiver. It should be mentioned that this is the first attempt to generate wireless signal capacities of up to $40 \mathrm{~Gb} / \mathrm{s}$. Record wireless capacity of up to $40 \mathrm{~Gb} / \mathrm{s}$ has been achieved using this technique. The proposed technique for generation and demodulation is tolerant to dispersion and incorporates only baseband components which enable easy integration with current optical access networks.

\section{ACKNOWLEDGMENT}

This work has been supported by the European Commission FP7 under Network of Excellence project EUROFOS (22402) www.eurofos.eu. R. Sambaraju acknowledges the Genralitat Valencia for the scholarship program Santiago Grisolia. Vedran Furtula DTU•Electro, Sonja Bojanic from Anritsu Denmark and Marco Martinez from Tektronix Spain are also acknowledged.

\section{REFERENCES}

[1] J. Wells, "Faster than fiber: the future of multi-Gb/s wireless," IEEE Microw. Mag, vol. 10, no. 3, pp. 104-112, 2009.

[2] C.-T. Lin, E.-Z.Wong, W.-J. Jiang, P.-T. Shin, J. Chen, and S. Chi, "28$\mathrm{Gb} / \mathrm{s}$ 16-QAM OFDM radio-over- ber system within 7-GHz licensefree band at $60 \mathrm{GHz}$ employing all-optical up-conversion," in Conference on Lasers and Electro-Optics, 2009 and 2009 Conference on Quantum electronics and Laser Science CLEO/QELS 2009., 2009. Postdeadline Paper CPDA8.

[3] M. Weiss, A. Stohr, F. Lecoche, and B. Charbonnier, "27 Gbit/s photonic wireless $60 \mathrm{GHz}$ transmission system using 16-QAM OFDM," in International Topical Meeting on Microwave Photonics, 2009. MWP '09.PostDeadline Paper.

[4] S. Chandrasekhar and X. Liu, "Experimental investigation on the performance of closely spaced multi-carrier PDM-QPSK with digital coherent detection.," Optics express, vol. 17, 2009, pp. 21350-61.

[5] D. Zibar, R. Sambaraju, R. Alemany, A. Caballero, J. Herrera, and I.T. Monroy, "Radio-Frequency Transparent Demodulation for Broadband Hybrid Wireless-Optical Links," IEEE Photonics Technology Letters, vol. 22,2010 , pp. $784-786$ 